\title{
Time-Resolved Collapse And Revival Of The Kondo State Near A Quantum Phase Transition
}

\section{Doctoral Thesis}

Author(s):

Wetli, Christoph

Publication date:

2017

Permanent link:

https://doi.org/10.3929/ethz-b-000161408

Rights / license:

In Copyright - Non-Commercial Use Permitted 
DISS. ETH NO. 24272

\section{TIME-RESOLVED COLLAPSE AND REVIVAL OF THE KONDO STATE NEAR A QUANTUM PHASE TRANSITION}

A thesis submitted to attain the degree of DOCTOR OF SCIENCES of ETH Zurich (Dr. sc. ETH Zurich)

Presented by CHRISTOPH MARTIN WETLI

MSc ETH in Physics, ETH Zurich

Born on September 30, 1988

Citizen of Zurich ZH, Switzerland and Oberwil-Lieli AG, Switzerland

Accepted on the recommendation of

Prof. Dr. Manfred Fiebig, Examiner Prof. Dr. Johann Kroha, Co-examiner Prof. Dr. Manfred Sigrist, Co-examiner Prof. Dr. Danilo Pescia, Co-examiner Prof. Dr. Ralph Spolenak, Chair 


\section{Christoph Wetli}

Heimensteinstrasse 13

8472 Seuzach

Switzerland

e-mail: christoph.wetli@mat.ethz.ch

All rights reserved

No part of this book may be reproduced, stored in a retrieval system, or transmitted in any form or by any means, electronic, mechanical, photocopying, microfilming, recording, or otherwise, without prior written permission of the author. 


\section{Declaration of originality}

I hereby declare that the following submitted thesis is original work, which I alone have authored and which is written in my own words.

Title: $\quad$ Time-resolved collapse and revival of the Kondo state near a quantum phase transition

Author: Christoph Wetli

Supervisor: $\quad$ Prof. Dr. Manfred Fiebig

With my signature I declare that I have been informed regarding normal academic citation rules and that I have read and understood the information on "Citation etiquette". The citation conventions usual to the discipline in question here have been respected.

Furthermore, I declare that I have truthfully documented all methods, data, and operational procedures and not manipulated any data. All persons who have substantially supported me in my work are identified in the text and in the acknowledgments.

The above work may be tested electronically for plagiarism.

Zurich, June 15, 2017 



\section{Abstract}

A major goal of modern condensed matter physics is to develop new classes of materials showing novel states of matter. By coupling the magnetic and electronic properties, materials with truly astonishing properties can emerge, such as high-temperature superconductivity, colossal magneto-resistance, spintronic and multiferroic materials.

These exotic states of matter are often found close to magnetic instabilities, hence in a regime where the quantum fluctuations of the magnetic and electric ordering are strongly coupled. The so-called heavy-fermion materials (sometimes also called heavy-electron materials) are at the very limit of magnetic instabilities and therefore depict an ideal test-bed for the understanding of interactions between electronic and magnetic ordering. In the phase diagram the heavy-fermion states are often surrounded by phases which show exquisite properties. Because of the complexity of these phase diagrams, they are still under intense debate.

The term ,heavy fermion' was introduced in the late 1970s for a new class of inter-metallic compounds which have a huge electron density at the Fermi surface, orders of magnitude larger than for conventional metals. Nevertheless, these heavy fermions could be described with conventional models derived for metals, but the effective electron mass has to be greatly increased.

The driving mechanism responsible for the heavy-fermion ground state is called the Kondo effect. The Kondo effect describes the interaction between a localized magnetic moment embedded in a sea of conduction electrons. The Kondo effect predicts that the localized magnetic moment will be screened by surrounding conduction electrons. The magnetically screened orbital is called a Kondo singlet. As the screening effect of the localized moment is continuously growing with decreasing temperature, the system is described by its Kondo temperature $T_{\mathrm{K}}$.

In the 1980s, the discovery of inter-metallic antiferromagnetism has boosted the interest in heavy-fermion compounds. Most heavy-fermion materials can be tuned by an external parameter to undergo a magnetic phase transition. Hence, a singularity in the phase diagram emerges for a critical control parameter value. In this ,quantum critical' regime of the phase diagram the electric and magnetic properties are profoundly changed with respect to the heavy-fermion phase and the magnetically ordered ground state. Since this exquisite quantum critical regime represents a magnetic instability, the emergence of complex ground states such as unconventional superconductivity, Mott insulators or topologically non-trivial states is predestined. The groundbreaking discovery of superconductivity in magnetic systems did not only boost the interest in the quantum critical regime, but also cleared the way for the superconducting cuprates.

A frequently used experimental ansatz to characterize quantum critical regimes are transport measurements which are sensitive to the number of charge carriers at the Fermi surface. Furthermore, scanning tunneling spectroscopy, as well as photoemission experiments are performed to extract band structure information, 
as well as information about the spatial coherence. Furthermore, also optical spectroscopy techniques are used to investigate the electromagnetic response. In order to reach a sufficiently good energy resolution (defined by the Kondo temperature), Fourier spectroscopy techniques (using continuous-wave sources) are the technique of choice.

Within the framework of this thesis the author investigates a novel aspect in strong correlation physics: the temporal coherence aspects and the intrinsic revival time of strong correlations in Kondo-lattice systems. To investigate the formation dynamics of strongly correlated systems, the author uses a temporally confined terahertz light pulse with suitably low photon energy to efficiently excite the Kondo singlet. In contrast to photoemission experiments, the photoexcited electrons remain inside the system and allow a re-establishment process of the strongly correlated ground state. Because the photon energy of terahertz radiation is small, the photoexcited electrons have only a tiny phase space into which they can scatter. Hence, an extraordinarily long lifetime of the quasiparticles is expected. The time-resolved terahertz experiment presented here does not only reveal the long lifetime of the excited state but also reveals the strikingly different time evolution between strongly and weakly correlated systems. The different time scales allow a virtually background-free observation of the revival of the strong correlation after a temporally strongly confined perturbation. This novel ansatz gives simultaneously access to two quantities describing the dynamics of a strongly correlated Kondo lattice: the Kondo spectral weight (a measure for the probability of the very existence of quasiparticles) as well as the spectral width of the Kondo resonance peak (corresponding to the effective Kondo temperature $T_{\mathrm{K}}^{*}$ ).

Pulsed terahertz sources are a promising tool to investigate the time evolution of strong correlation physics. This versatile experimental ansatz is not only ideal for investigating quantum phase transitions, but can also be used for other strongly correlated ground states such as in topological insulators, Mott insulators, Kondo insulators or unconventional superconducting materials. The work at hand is restricted to the observation of the ,revival ${ }^{6}$ of the strongly correlated Kondo ground state and investigations of the associated quantum critical regime. The presented experimental results reveal an overall behavior expected for unconventional quantum criticality. However, thanks to the simultaneous monitoring of the two key features of the Kondo effect, some aspects were found which ask for a tweaking of our current understanding of unconventional quantum criticality. 


\section{Zusammenfassung}

In der Festkörperphysik spielt die Erforschung und Entwicklung neuartiger Materialien mit speziellen Eigenschaften eine zentrale Rolle. Diese speziellen Materialeigenschaften können durch die Kopplung magnetischer und elektrischer Eigenschaften erzeugt werden wie, beispielsweise Hochtemperatursupraleiter, Materialien mit einem kolossalen magnetoresistiven Effekt, spintronische Systeme oder auch Multiferroika. Diese exotischen Materiezustände werden oft in unmittelbarer Nähe von magnetischen Instabilitäten gefunden, da dort die sogenannten Quantenfluktuationen der magnetischen und elektrischen Ordnung stark gekoppelt sein können. Die sogenannten Schwer-Fermion-Systeme ermöglichen einen idealen Zugang zu diesen magnetischen Instabilitäten und bilden daher eine optimale Umgebung um deren Eigenschaften zu erforschen. Die Schwer-Fermion-Phasen sind oft von exotischen Grundzuständen umgeben, die zunächst unerwarteterweise auftreten. Diese komplexen Phasendiagramme sowie das Erscheinen neuartiger Grundzustände sind die Hauptgründe für das Interesse an Schwer-Fermion-Systemen.

Der Begriff des Schwer-Fermions wurde eingeführt um eine neue Art von Zwischenmetallverbindungen $\mathrm{zu}$ beschreiben, die eine stark überhöhte Zustandsdichte an der Fermikante besitzen. Die Zustandsdichte in Schwer-Fermion-Systemen kann, im Vergleich zu herkömmlichen Metallen, wie beispielsweise Kupfer, um Grössenordnungen erhöht sein. Nichtsdestotrotz können diese Schwer-Fermion-Metalle mit der herkömmlichen Metalltheorie beschrieben werden, wobei jedoch die effektive Masse stark renormiert werden muss. Der treibende Effekt hinter diesem Grundzustand ist der sogenannte Kondo-Effekt. Der Kondo-Effekt beschreibt die Wechselwirkung eines lokalen, magnetischen Moments mit den ihm umgebenden Leitungselektronen. Der Effekt prognostiziert, dass das lokale magnetische Moment durch die sogenannten Spins der Leitungselektronen bei ausreichend tiefen Temperaturen abgeschirmt wird. Die für diesen Effekt relevante Temperaturskala ist durch die Kondo-Temperatur $T_{\mathrm{K}}$ gegeben.

Mit der Entdeckung des Antiferromagnetismus in den Zwischenmetallverbindungen wurden Schwer-Fermion-Systeme schlagartig für Anwendungen interessant. Die Tatsache, dass ein Schwer-Fermion-System mittels eines externen Parameters in einen disjunkten, magnetisch geordneten Grundzustand gebracht werden kann, ist gleichbedeutend mit der Existenz einer Art Singularität im Phasendiagramm. In diesem sogenannten, quantenkritischen" Regime des Phasendiagramms verändern sich die magnetischen sowie die elektrischen Eigenschaften des Systems grundlegend. Daher treten in unmittelbarer Nähe oft stark korrelierte Phasen auf, wie zum Beispiel unkonventionelle Supraleitung, Mott Isolation oder topologische Grundzustände. Mit der bahnbrechenden Entdeckung von Supraleitung in magnetischen Systemen wurde nicht nur das Interesse an Schwer-Fermion-Systemen geweckt, sondern es wurde auch ein Weg für die Entwicklung und Entdeckung neuartiger Hochtemperatursupraleiter aufgezeigt. 
Experimentell werden Schwer-Fermion-Systeme sowie das quantenkritische Regime oft mittels Transportmessungen untersucht, da diese sensitiv auf Zustandsänderungen an der Fermikante sind. Die räumlichen Kohärenzphänomene von Schwer-Fermion-Systemen können mittels Rastertunnelmikroskopie untersucht werden. Es sind auch Bandstrukturuntersuchungen mittels energetisch hochaufösender Photoemissionsexperimente möglich. Um bei optischen Spektroskopiemethoden eine ausreichende spektrale Auflösung zu haben, wird oft auf die etablierte Fourier-Spektroskopie zurückgegriffen.

Im Rahmen der vorliegenden Arbeit hat der Autor den Aspekt der zeitlichen Kohärenz von Schwer-Fermionen untersucht. Die Experimente des Autors zeigen eine intrinsische Aufbauzeit des stark korrelierten Grundzustandes in Kondo-Gittern. Um diese Aufbauzeit zu messen, wird das System mittels eines ultrakurzen Terahertzpulses aus dem Gleichgewicht gebracht. Die Photonenenergie ist zwar ausreichend gross um den Kondo-Grundzustand anzuregen, jedoch wird die Probe (im Gegensatz zu Photoemissionsexperimenten) nicht ionisiert. Das bedeutet, dass diese schwach angeregten Elektronen den Schwer-Fermion-Zustand auf einer intrinsischen Zeitskala wiederherstellen können. Da die Photonenenergie der Terahertzstrahlung klein ist, haben die angeregten Elektronen nur einen sehr kleinen Phasenraum, in welchem sie streuen können. Diese stark eingeschränkte Streuung erklärt, weshalb eine lange Lebensdauer der entsprechenden Quasiteilchen erwartet wird. Das hier vorgestellte Experiment demonstriert nicht nur diese lange Lebensdauer der angeregten Zustände, sondern zeigt auch die fundamental unterschiedliche Relaxationsdynamik zwischen stark und schwach korrelierten Systemen auf. Die unterschiedlichen Zeitskalen erlauben es, nahezu hintergrundfrei, den intrinsischen Wiederaufbau der starken Korrelation zu beobachten, nachdem diese kontrolliert zerstört wurde. Der zeitaufgelöste Ansatz hat den weiteren Vorteil, dass gleichzeitig zwei relevante Grössen gemessen werden können: das Kondo-Spektralgewicht (ein Mass für die Wahrscheinlichkeit, dass Quasiteilchen existieren) sowie die Breite der Kondo-Resonanz an der Fermikante, was der Kondo-Temperatur entspricht. Diese zwei Grössen sind besonders in der Nähe von magnetischen Instabilitäten von grossem Interesse, da dort oftmals der Kollaps des etablierten Teilchenbildes erwartet wird.

Generell sind gepulste Terahertzquellen ein ideales Werkzeug um die Zeitentwicklung stark korrelierter Phänomene zu untersuchen. Gepulste Terahertzexperimente können sehr vielseitig eingesetzt werden: zur Untersuchung von Quantenkritikalität, aber auch für fundamental andere stark korrelierte Systeme wie beispielsweise topologische Isolatoren, Mott- und Kondo-Isolatoren oder auch für unkonventionelle Supraleiter. Die vorliegende Arbeit beschäftigt sich mit der dynamischen Untersuchung und der Formierung des stark korrelierten Kondo-Zustandes sowie des damit verbundenen quantenkritischen Regimes. Die hier vorgestellten Resultate verifizieren das erwartete, unkonventionell quantenkritische Verhalten in mehreren entsprechenden Schwer-Fermion-Systemen. Ausserdem konnte der Autor bisher unbekannte Aspekte der Quantenkritikalität experimentell aufgezeigt. 


\section{Acknowledgment}

I would like to pay tribute to the countless people who supported me during my time as a doctoral student and who helped me to successfully graduate. During my time at ETH Zurich, I improved my technical and physical knowledge tremendously thanks to great lectures and the countless informal discussions I had with so many of you (lecturers, staff, technicians, scientists, colleagues etc.). Therefore, I would like to thank all of you, who contributed to this life-changing experience. In particular and explicitly, I would like to thank the following people:

I thank my supervisor Prof. Manfred Fiebig for all his support, guidance and trust. I would like to thank you, Manfred, for your passion, support, enthusiasm and all the fruitful discussions. I learned and benefited a lot from your experience throughout the last couple of years.

My deepest gratefulness goes to Prof. Johannes Kroha who proposed my experiment. Hans, you always believed in the success of this project. I thank you for the countless discussions in which you patiently explained the comprehensive theoretical background of Kondo physics to me. Furthermore, I deeply appreciate your decision to spend your sabbatical with us at ETH Zurich.

Furthermore, I would also like to thank Prof. Manfred Sigrist for your help in understanding the complex theoretical framework of strong correlation physics. You and your excellent lectures (and lecture notes) sparked my interest for strongly correlated phenomena and solid state physics in general.

I also deeply appreciate the support of Prof. Danilo Pescia. You convinced me to take my doctorate in the group of Manfred and you always lend me an ear if I had a question about anything. It was your lecture about group theory in solid state physics which opened my eyes to the world of symmetries. And of course you introduced myself to the fascinating world of magnetism.

In addition, I would further like to thank Dr. Tobias Kampfrath and Dr. Tim Huber, who shared their outstanding knowledge in T-ray optics with me. Your countless and fruitful ideas helped me to realize the terahertz spectrometer. And thank you Tobias for visiting us in Zurich and for presenting the groundbreaking research of you and your team.

Further, I would like to thank Prof. Hilbert von Löhneysen, Prof. Cornelius Krellner, PD Dr. Oliver Stockert and Kristin Kliemt for providing the samples. Without your high-quality heavy-fermion samples my thesis would not have been possible.

I would also like to thank Prof. Philipp Gegenwart and Prof. Hilbert von Löhneysen for inviting me as guest speaker for Physics Colloquia to their universities. I feel very privileged and will always keep this great experience in good memory.

Also many thanks to Prof. Peter Wachter and Prof. Leonardo Degiorgi, who inexorably tried to organize differently doped cerium copper samples or other 
Kondo-lattice systems which could be suitable for the time-resolved terahertz experiments.

I would like to thank all the other supporters of the Ferroic team. You always supported me throughout my thesis and were always available for technical and scientific discussions. I would like to thank explicitly my colleagues Christian Tzschaschel, Jannis Lehmann, Ehsan Hassanpour Yesaghi and Dr. Shovon Pal, who patiently read my thesis and helped me to make it as perfect as possible.

A very special thanks go to my office mates Ehsan Hassanpour Yesaghi, Dr. Jakob Schaab, Dr. Matthias Hudl, Dr. Stefan Günther and Dr. Martin Lilienblum, with whom I spent several years in the same office. I will keep the countless and fruitful discussions in great memory. I enjoyed the time with you very much.

And of course, last but definitely not least, I also would like to pay tribute to my personal environment, my parents Esther and Martin, my sister Andrea and my lovely girlfriend Daniela and the entire Fognini family. All of you believed in the success of my doctorate. You kept me motivated throughout the entire time as a doctoral student. I really appreciate your moral assistance.

Thank you so much! 


\section{Contents}

1. Introduction 1

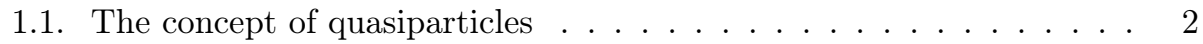

1.2. Kondo effect vs. Kondo-lattice effect . . . . . . . . . . . . . 3

1.3. Quantum phase transitions . . . . . . . . . . . . 5

1.4. Prospects for this thesis . . . . . . . . . . . . . 6

2. Kondo physics - theoretical background 9

2.1. The difference: weak vs. strong correlation . . . . . . . . . . . 10

2.2. The Fermi-gas approach towards weak correlation . . . . . . . . 11 2.2.1. The non-interacting Fermi gas . . . . . . . . . . 11

2.2.2. Perturbative treatment of Coulomb interaction in a Fermi gas 14

2.3. Fermi-liquid theory . . . . . . . . . . . . . . . . . . 14

2.4. The Kondo-impurity effect . . . . . . . . . . . . . . . . . 16

2.4.1. Induced spin-polarization in conduction electrons . . . . . . 16

2.4.2. The Kondo effect in dilute alloys . . . . . . . . . . . . . 18

2.5. The Anderson Hamiltonian . . . . . . . . . . . . . . . . . 21

2.5.1. The Kondo resonance emerging at the Fermi surface . . . . . 22

2.6. Quasiparticle lifetimes and spectral weight . . . . . . . . . 24

2.7. Crystal-field satellites and high-temperature Kondo effect . . . . . 27

2.7.1. Crystal-field splitting . . . . . . . . . . . . . 28

2.7.2. High-temperature Kondo effect . . . . . . . . . . . 30

3. Quantum phase transitions - theoretical background 31

3.1. Thermally-driven second-order phase transitions and universality

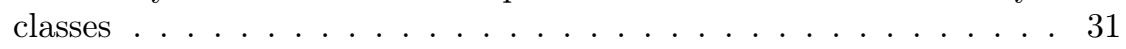

3.2. Quantum phase transitions in heavy-fermions . . . . . . . . . 32

3.3. Different quantum critical scenarios . . . . . . . . . . . . . 34

3.3.1. Conventional quantum criticality . . . . . . . . . . 34

3.3.2. Unconventional quantum criticality . . . . . . . . . . 36

4. Heavy-fermion systems and experimental approaches 39

4.1. $\mathrm{CeCu}_{6-x} \mathrm{Au}_{x} \ldots \ldots \ldots \ldots \ldots \ldots \ldots \ldots \ldots$

4.1.1. The basic alloy: $\mathrm{CeCu}_{6} \ldots \ldots \ldots \ldots$. . . . . . . . . .

4.1.2. Au doping: $\mathrm{CeCu}_{6-x} \mathrm{Au}_{x} \ldots \ldots \ldots$. . . . . . . . . 40

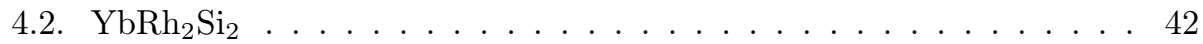

4.3. Experimental techniques . . . . . . . . . . . . . . . . 43

4.3.1. Transport measurements . . . . . . . . . . . . . . 44

4.3.2. Elastic neutron scattering . . . . . . . . . . . 47 
4.3.3. Scanning tunneling microscopy ... . . . . . . . . . . 48

4.3.4. Photoemission spectroscopy . . . . . . . . . . . 52

5. THz time-domain spectroscopy

5.1. Overview ...................... 555

5.2. Coherent terahertz radiation and their outstanding properties . . . . 57

5.3. Experimental setup . . . . . . . . . . . . . . 58

5.3.1. In detail description of the $\mathrm{THz}$ setup . . . . . . . . . . . . 60 60

5.4. Generation of $\mathrm{THz}$ pulses . . . . . . . . . . . . . . . 63

5.4.1. Optical rectification process to generate $\mathrm{THz}$. . . . . . . 65

5.4.2. Orientation-dependent optical rectification process in $\mathrm{ZnTe}$. 67

5.5. Phase-sensitive detection of coherent $\mathrm{THz}$ radiation . . . . . . . . . . 69

5.5.1. Measuring the birefringence of the electro-optic sampling

crystal .................... . . 71

5.6. Carrier-envelope phases and transform limitations in wave packets . 72

5.7. Extraction of the non-equilibrium response . . . . . . . . . . 73

5.8. Investigating metallic systems - reflection geometry . . . . . . . . . . 74

5.8.1. Drude-Lorentz model for metals . . . . . . . . . . . . . . . 74

5.9. Experimental limitations in the time domain . . . . . . . . . . 76

5.9.1. Temporal sampling rate . . . . . . . . . . . . . . 76

5.9.2. Finite trace effects . . . . . . . . . . . . . . . 77

5.10. Efficiency aspects and estimation of the electric field strengths . . . 79

6. Heavy-fermion dynamics in $\mathrm{CeCu}_{6-\mathrm{x}} \mathrm{Au} \mathrm{u}_{\mathrm{x}}$ and $\mathrm{YbRh}_{2} \mathrm{Si}_{2}$

6.1. Goals for time-resolved measurements of heavy fermions . . . . . . . 81

6.2. Heavy-fermion signature in $\mathrm{THz}$ time traces in $\mathrm{CeCu}_{6-\mathrm{x}} \mathrm{Au}_{\mathrm{x}}$. . . . . 82

6.3. Phenomenological understanding of heavy-fermion signature . . . . . 84

6.4. Characteristic strong correlation dynamics . . . . . . . . . . . . . . 85

6.4.1. Phenomenological rate equation . . . . . . . . . . . . 85

6.4.2. Dynamical mean-field theory . . . . . . . . . . . . 89

6.5. Conformity of experiment and rate-equation ansatz . . . . . . . . 91

6.5.1. Proof of phase coherence . . . . . . . . . . . . . . . 93

6.5.2. Kondo-echo intensity versus THz pump intensity . . . . . . . 95

6.6. Frequency response in $\mathrm{CeCu}_{5.9} \mathrm{Au}_{0.1} \ldots \ldots$. . . . . . . . . . . . . . . . . . . . .

6.6.1. Spectrum of full time trace . . . . . . . . . . . . . 96

6.6.2. Spectrum of the Kondo echo . . . . . . . . . . . . . . . . 97

6.6.3. Kondo response function . . . . . . . . . . . . . . . . . . 98

6.7. High-temperature Kondo effect in $\mathrm{CeCu}_{5.9} \mathrm{Au}_{0.1} \ldots \ldots$. . . . . . . . . 99

6.8. Coherent heavy-fermion behavior in $\mathrm{YbRh}_{2} \mathrm{Si}_{2}$. . . . . . . . . . . 102

7. Quantum phase transition in heavy fermion $\mathrm{CeCu}_{5.9} \mathrm{Au}_{0.1}$

7.1. Kondo-echo analysis . . . . . . . . . . . . . . . . . . . 106

7.1.1. Temperature dependence of the Kondo scale $T_{\mathrm{K}}^{*}$. . . . . . . 106

7.1.2. Temperature-dependent Kondo spectral weight . . . . . . . . 107 
Bibliography

A. THz generation

A.1. Analytical calculation of a $\mathrm{THz}$ pulse generated by optical rectification 127

A.2. Experimental: optical-rectification process in $\mathrm{ZnTe}(110)$. . . . . . . 128

B. $\mathrm{THz}$ detection

B.1. Analytical description of the electro-optical sampling . . . . . . . . . 131

B.2. Analytical description of birefringence measurement . . . . . . . . 133

C. Water absorption

C.1. Molecular modes of $\mathrm{H}_{2} \mathrm{O} \ldots \ldots$. . . . . . . . . . . . 1355

D. Fabry-Perot etalon effects 137

D.1. Cryostat windows . . . . . . . . . . . . . . . . . . . . 137

D.2. Challenges in time-resolved measurements . . . . . . . . . . . . . . 138

E. Rate equation describing the heavy-fermion dynamics 141

F. Resistivity analysis $\mathbf{1 4 3}$

F.1. Kondo lattice vs. Kondo impurity at low temperatures . . . . . . . . 144

\section{G. Data overview}

H. Spatial resolution in $\chi^{(2)}$-processes

H.1. Analytical calculation of integrated SHG intensity for large domains 148

H.2. Analytical calculation of integrated SHG intensity for small domains 150

H.3. Final remarks . . . . . . . . . . . . . . . . . . . . . . . . . 152

Scientific achievements 153

Publications . . . . . . . . . . . . . . . . . . . . . . . . . . 153

Invited talks . . . . . . . . . . . . . . . . . . . 155

Conference contributions . . . . . . . . . . . . . . . . . 155

Curriculum vitae 



\section{Introduction}

The term ,heavy fermion' was created in the late 1970s in order to describe a newly discovered group of intermetallic compounds, which show a hugely increased specific heat capacity, which scales with the density of states at the Fermi surface. The first discovered heavy fermion compound was $\mathrm{CeAl}_{3}$ [1. In the following years, many other systems were identified 2,12 . The interest into heavy fermions was immensely boosted by the discoveries of various exquisite ground states such as superconductivity [2, 4, 6], antiferromagnetism [13 -15] and insulating phases in close proximity of the heavy-fermion phase. In order to understand the drastic consequences of these discoveries, a brief introduction to heavy-fermion compounds has to be given.

A heavy-fermion system consists of a periodic lattice of actinide or lanthanide magnetic ions embedded in a metallic environment. Non-heavy-fermion rare-earth compounds are known to have preferably an antiferromagnetically ordered ground state at sufficiently low temperatures. Under the right circumstances and because the conduction electrons carry magnetic moments, though, the localized magnetic ions can be magnetically screened by the conduction electrons. This screening of the magnetic moments will consequently prevent the system from showing any magnetically ordered ground state. Hence, this screening effect is melting down the magnetic order. This interaction mechanism between the localized magnetic moments and the conduction electrons is called the Kondo effect [16 18], which will lead to a full screening of the magnetic moments at sufficiently low temperatures. Since the exchange interaction between the conduction electrons and the localized magnetic moment is continuously growing with decreasing temperature, an intrinsic temperature scale, which is defined by the so-called Kondo temperature $T_{\mathrm{K}}$, specifies the energy range on which the Kondo screening takes place. As a direct consequence of the Kondo screening the electrical resistivity will increase again below the Kondo temperature. The reason for this increase is the enhanced scattering potentials as magnetic moments become dressed by the conduction electrons.

A heavy-fermion compound consists of a periodic magnetic sublattice and is often referred to as a Kondo-lattice compound [19]. The Kondo effect with magnetic moments placed in a dense lattice can also be understood as a partial reduction of the localized magnetic moments as they start to form mobile excitations at the Fermi surface. These mobile excitations at the Fermi surface drastically enhance the effective mass (therefore the term ,heavy fermion ${ }^{*}$ ) and thus induce profound changes in transport properties. 
Many heavy fermions can be tuned by an external parameter to undergo a magnetic phase transition 20,22 . This groundbreaking discovery has some profound effects on the phase diagram, since the heavy fermion and magnetic ordering regime are disjunct ground states. Hence, the system is expected to be in a singularity state for a critical tuning parameter $13-15$. Close to this singularity, a so-called quantum critical regime emerges. Here, the phase diagram is dominated by this magnetic instability behavior, causing profoundly modified properties of the system, which are generally called non-Fermi-liquid behavior. Often, novel phases emerge in the presence of such magnetic instabilities which makes phase diagrams in the quantum critical regime highly interesting from a very general point of view.

\subsection{The concept of quasiparticles}

Describing an ensemble of strongly interacting particles is one of the most complex problems in modern condensed matter physics. An approach towards solving this problem was performed by the Soviet theoretician Landau, who suggested to treat a system as a ground state accompanied with elementary, quantized excitations. These quantized excitations are called quasiparticles since the coherent part of these excitations follow a well-defined dispersion relation, which pinpoints the particle-like nature (see section 2.6). As the goal is to describe a ground state, only the energetically lowest excited states are taken into account for determining the material properties in the quasiparticle approach. Based on this concept of quasiparticles, Landau developed the so-called Fermi-liquid theory in 1957 [23 25]. This phenomenological theory describes the physical properties of an interacting fermion system, where the interactions amongst the quasiparticles is small. Fermi-liquid theory can be adiabatically derived from the non-interacting fermion systems (so-called Fermi gas). This theory has proven to be a remarkably powerful tool describing a vast amount of phenomena, even if strong electron-electron interactions are present. Fermi-liquid theory allows for a phenomenological understanding and a mathematical description of these complex states of matter.

The theory's main assumption is that the physical properties are mostly determined by the quasiparticle interactions. Fermi-liquid behavior can be found at sufficiently low temperatures, for example, in normal metals as well as in heavy-fermion compounds. In the latter case, the effective electron mass $m^{*}$ has to be strongly renormalized. The characteristic temperature scale in normal metals to observe Fermi-liquid behavior is defined by the Fermi-surface temperature $T_{\mathrm{F}}$. The qualitative behavior of many physical properties in Fermi-liquid theory is equal to the non-interacting Fermi gas. In Landau Fermi-liquid theory the effective mass of the quasiparticles is independent of environmental conditions such as temperature, pressure, magnetic field or other external parameters. Furthermore, the effective mass is the only tuning parameter in the Fermi-liquid description.

Fermi-liquid behavior can only be observed at sufficiently low temperatures because thermal fluctuations should be small in order to fulfill the assumption specified 
above. For too high temperatures, the Fermi-liquid theory is no longer applicable as other scattering processes start to dominate the ,high'-temperature behavior (for example the electron-phonon scattering leads to a $T^{5}$-term in the electrical resistivity). Note that ,low' temperature in this context means that the boundary between occupied and unoccupied states (called the Fermi surface) has to be energetically sharp. In this limit, the energy and momentum conservation is strongly suppressing the scattering processes due to the tiny available phase space (also called Pauli blocking). This reduced scattering probability causes higher-order terms in thermodynamic quantities with respect to non-Fermi-liquid behavior 1 In contrast to a normal metal, in heavy-fermion systems the Kondo effect has to establish first, before eventually a ,heavy'-Fermi-liquid behavior can emerge. Hence, the relevant temperature scale for heavy-fermion behavior is the Kondo temperature $T_{\mathrm{K}}$. Despite the different energy scales of heavy fermions and ordinary metals, the systems show qualitatively a similar behavior.

Despite the huge success of the quasiparticle concept, there are numerous examples of systems which do not comply with Fermi-liquid theory. The limitation of Landau Fermi-liquid theory is specified by the so-called Pomeranchuk stability condition 2 Such a non-Fermi-liquid behavior manifests, for instance, in the scaling behavior of thermodynamic properties, where the experimentally observed critical exponents cannot be explained using the quasiparticle concept. It is therefore believed that the non-Fermi-liquid behavior of matter is closely linked to exotic states, where even the very concept of particles could break down. Non-Fermi-liquid behavior is often observed in the vicinity of magnetic instabilities as they, for example, occur in phase diagrams between a heavy fermion and a magnetically ordered state. In this regime of the phase diagram, the magnetic and electronic degrees of freedom are strongly coupled [10, 13, 28, 30].

\subsection{Kondo effect vs. Kondo-lattice effect}

In 1934 the Kondo effect was experimentally discovered by the Dutch physicist de Haas 31. The goal of his studies was to investigate the low-temperature behavior of various metals. Since his samples had (magnetic) Fe impurities, he discovered an unexpected minimum the temperature-dependent electrical resistivity. It turned out that the magnetic Fe impurities and the cohesive Kondo effect are responsible for the puzzling low-temperature resistivity behavior reported by him. The origin of this extraordinary metallic behavior remained unknown for about 30 years.

In 1964 the Japanese theoretician Kondo described the influence of magnetic impurities on the conductivity of metals in terms of scattering theory, nowadays called the Kondo effect [17]. Kondo derived his theory using the

\footnotetext{
${ }^{1}$ For example electrical resistivity: a $T^{2}$-term is expected for Fermi liquids, whereas non-Fermi liquids typically show linear temperature scaling behavior.

${ }^{2}$ The Pomeranchuk stability condition states that the quasiparticle approach is only valid for positive interaction terms amongst the quasiparticles and as long as they remain above a threshold value [26] 27.
} 
,s-d Hamiltonian' and by taking second-order perturbation terms into account, see section 2.4.2. These second-order perturbations give rise to an additional logarithmic and strongly temperature-dependent term in the resistivity $R$ :

$$
R=R_{\text {Born }} \cdot\left(1+\frac{2 J \rho}{N} \ln \left(\frac{k_{\mathrm{B}} T}{W}\right)\right),
$$

where $R_{\text {Born }}$ is the resistivity obtained using the first Born approximation. $J$ represents the spin-exchange interaction, $\rho$ the density of conduction electrons, $N$ the density of magnetic impurities, $k_{\mathrm{B}}$ the Boltzmann constant, $T$ the temperature and $2 W$ the width of the conduction band.

The logarithmic contribution of the Kondo effect is triggered by the spin-exchange interaction between the localized magnetic moments and the delocalized electrons of the metal. These screened localized magnetic moments act as strong elastic scatterers for the remaining mobile electrons and ultimately leads to an increase in the electrical resistivity. The localized magnetic moments are located well below the Fermi energy, but due to resonant scattering processes 3 some fraction of its spectral weight is transferred to the Fermi surface, forming a resonance peak. As a heavy-fermion system consists of a lattice with magnetic ions, the Kondo effect may be alternatively regarded as mobile excitations of the localized magnetic moments. Such mobile excitation states cause a spectral weight shift of the atomic orbital carrying the magnetic moment towards the Fermi surface. Since the resonance and the conduction band avoid a band crossing, a hybridization gap establishes. This causes a shallow band structure in close proximity to the Fermi surface and therefore most physical properties of Kondo systems have to be strongly renormalized to match the Fermi-liquid behavior. As in Fermi-liquid theory the effective mass $m^{*}$ is the only fitting parameter, heavy fermions show Fermi-liquid behavior with a hugely enhanced $m^{*}$. The details of the Kondo effect and heavy fermions are discussed in chapter 2.

Already in 1961, Anderson theoretically predicted that the periodic arrangement of magnetic impurities in a metal could lead to peculiar ground states [32]. Indeed, the Kondo physics can be tremendously enriched as the magnetic ions form a periodic lattice rather than single, decoupled magnetic impurities. This makes for the difference between Kondo-effect systems and the heavy-fermion compounds. A system with a periodic magnetic sublattice is also called a Kondo lattice, as the interaction amongst the magnetic impurities can no longer be neglected. It was this very perception that lead to the current interest in QPTs. The non-Fermi-liquid behavior in heavy fermions was only experimentally discovered in 1994 by von Löhneysen and co-workers [13. However, heavy-fermion systems attracted a lot of attention already before, triggered by the discovery of unconventional superconductivity in magnetic systems [2, 3, 33].

Despite the numerous experiments and theoretical discussions of Kondo lattices, QPTs and their phase diagrams remain puzzling and challenging until today.

\footnotetext{
${ }^{3}$ Resonance scattering does not ,cost' any energy.
} 
Furthermore and according to the authors knowledge, only little is known about the temporal coherence aspects of Kondo lattices.

\subsection{Quantum phase transitions}

Most heavy-fermion systems can be tuned by an external parameter to undergo a magnetic phase transition [13, 14, 34]. Such an external tuning parameter can be, for instance, pressure, a magnetic field or chemical doping. Since the heavy fermion and the magnetically ordered ground states are strictly separated in the phase diagram, the magnetic ordering temperature can be continuously driven to zero. At this very value of the tuning parameter, the phase diagram shows a singularity, at which the system undergoes a so-called a quantum phase transition (QPT) [10, 35]. For this critical value of the tuning parameter, the metallic behavior of the system is radically changed with respect to the behavior in the neighboring phases, revealing a non-Fermi-liquid behavior. A remarkable feature of the QPT is its influence towards higher temperatures. Despite the actual QPT being at $0 \mathrm{~K}$, its influence can be observed over a wide range of temperatures and well above the actual QPT. Since the very discovery of QPTs in heavy fermions, the question about what happens with the heavy fermions in the quantum critical regime has risen and remains largely unanswered until today. It is clear, though, that as the system enters the magnetically ordered phase, the Kondo screening has to be weakened such that the local magnetic moments at least partially can re-emerge. However, whether the Kondo screening breaks down locally or the breakdown is more spin-density-wave-like is a challenging question, especially since there are experimental examples for the different breakdown scenarios.

a

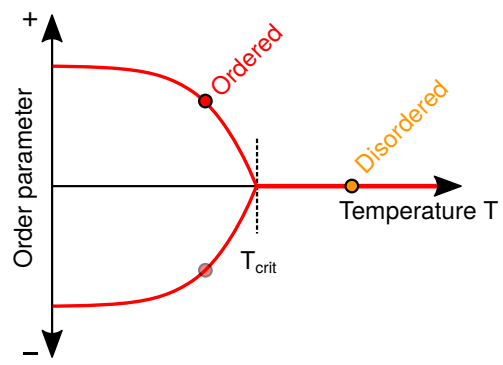

b

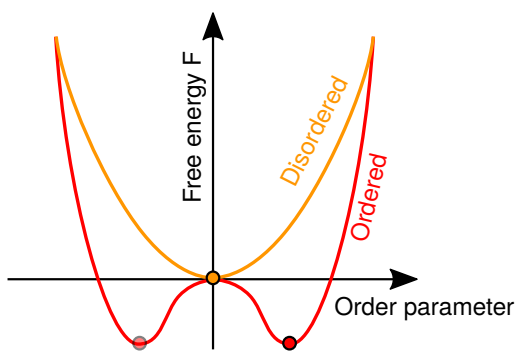

Figure 1.1.: (a) Temperature-dependent growth of an order parameter. Below the transition temperature $T_{\text {crit }}$ of a second-order phase transition, an order parameter grows gradually. (b) In Landau theory, the free energy $F$ changes its shape at the phase transition temperature: In the disordered state $\left(T>T_{\text {crit }}\right)$, the only minimum in $F$ can be found where the order parameter vanishes. In the ordered phase $\left(T<T_{\text {crit }}\right)$, there are two minima for two finite values of the order parameter. 
In contrast to QPTs, phase transitions at finite transition temperatures are driven by the thermal fluctuations. A second-order phase transition manifests in the growth of a so-called order parameter below a critical ordering temperature. Landau theory is a general theory for second-order phase transitions. It describes the system in terms of the thermodynamic free energy. As the system is driven into the ordered phase, thermal fluctuations lead to a divergence of the spatial and temporal correlation of the order parameter. However, the temperature window of the critically large coherence is narrowed down for decreasing transition temperatures. Therefore, a phase transition at $0 \mathrm{~K}$ is purely driven by so-called quantum fluctuations originating from Heisenberg's uncertainty principle (due to absence of thermal fluctuations).

QPTs are of tremendous interest and are subject to intense debates. Despite the inaccessibility of the QPT itself, the augury of the emergence of a QPT can be observed even at temperatures as high as $60 \mathrm{~K}$ in photoemission experiments 36. Furthermore, as a system is about to undergo a QPT, often suddenly a new strongly correlated phase emerges, before the QPT point is actually reached. The discovery of unconventional superconductivity close to the $\mathrm{QPT}$ in $\mathrm{CeCu}_{2} \mathrm{Si}_{2}$ was the first experimental observation of superconductivity close to a magnetic instability [2]. Generally, it is believed that in the vicinity of the QPTs many exotic states might be discovered. A detailed insight into the fascinating field of QPTs is given in chapter 3 .

\subsection{Prospects for this thesis}

The thesis at hand is dedicated to address the temporal coherence phenomena in strongly correlated systems. The experiments presented here will demonstrate that a picosecond far-infrared electromagnetic pulse can be used to drive a Kondo-lattice system into a carefully prepared non-equilibrium state, which allows us to observe the temporal emergence of the strongly correlated Kondo-lattice ground state (for the theory, see chapter 2 and for the experiments, see chapter 6). This approach to Kondo dynamics further gives a different perspective onto the world of QPTs (for the theory, see chapter 3 and for the experiment, see chapter 7].

In order to perform the time-resolved experiments on heavy-fermion compounds and QPTs, the author designed and built a low-temperature terahertz time-domain spectrometer (chapter 5) which operates in reflection geometry. The measurements and results on Kondo-lattice dynamics and quantum criticality shown in this thesis were performed using this self-built scientific instrument.

Generally speaking, using pulsed terahertz sources for investigating temporal coherence phenomena of strongly correlated systems is a promising ansatz [37. It enables a time-resolved point-of-view on strong correlation phenomena on the sub-picosecond time scale. In contrast to photoemission experiments, terahertz radiation is a more gentle probing technique as the electronic structure is only jiggled and the system is not ionized. Hence, experiments using pulsed terahertz 
sources could help to better understand strong correlation mechanisms. This ansatz is not only limited to Kondo systems, but could also help to understand the intrinsic ,revival' mechanisms of other strongly correlated systems such as Mott insulators, topological materials as well as unconventional superconductors. 



\section{Kondo physics - theoretical background}

Metallic compounds with magnetic impurity atoms show a minimum in the temperature-dependent electrical resistivity [17, 18, 31. This minimum in resistivity is caused by the exchange interaction between the conduction electrons and the localized magnetic sites, causing an electron cloud surrounding each impurity and screening its magnetic moment. This mechanism of conduction electrons quenching localized magnetic moments is called the Kondo effect and is strongly temperature dependent. The ,quenched' magnetic moments are commonly called Kondo singlets. The enhanced elastic scattering potential of the ,quenched' magnets manifests in the electrical properties of the system. Hence, and despite the difficulty of directly monitoring the Kondo singlets, their influence on macroscopic quantities is striking and can be barely overlooked. Various experimental techniques are dedicated to investigate different aspects of the Kondo effect. A summary over the most frequently used techniques is given in chapter 4

If the magnetic impurities are arranged on a periodic lattice, the Ruderman-Kittel-Kasuya-Yosida (RKKY) interaction starts to play a crucial role and opens the door to the huge field of quantum critical phenomena. The RKKY interaction is responsible for a long-range magnetic ordering, causing a ferromagnetic or antiferromagnetic arrangement of the localized magnetic moments. It describes the indirect exchange interaction between localized magnetic moments mediated by the spin polarization of the conduction electrons. Since the Kondo effect is locally screening the localized magnetic moments, the two mechanisms are in direct competition with each other, see section 2.4. This fine balance between the RKKY interaction and the Kondo effect allows for a continuous adjustment of the phase transition temperature, eventually culminating in a quantum phase transition (QPT), which occurs at $0 \mathrm{~K}$. The quantum critical aspects of QPTs are discussed in chapter 3 .

This chapter is dedicated to give an overview over the comprehensive theory of Kondo physics. Furthermore, a detailed understanding of the Kondo effect is required to understand its temporal coherence as experimentally demonstrated in chapter 6. More details about the Kondo effect, as well as about periodic Kondo lattices can be found, for example, in [27, 38 42], as they were the inspiration source for this chapter. 


\subsection{The difference: weak vs. strong correlation}

A solid structure can be understood as an ensemble of $N$ individual particles, which form a rigid body. In mathematical terms, this $N$-particle picture poses the problem of solving $N$ coupled partial differential equations 11 Since $N$ is of the order of $10^{23}$, this approach is not feasible. By neglecting the interactions amongst individual particles completely all scattering processes are ignored. Even such a simplified picture often correctly depicts the properties of solids, for example whether a crystal is metallic or insulating. One of the most prominent examples is the so-called Fermi-gas ansatz of the band structure model. The electron bands in a solid describe the eigenstates of electrons in a crystal which must have well-defined energy-momentum relations. In the case of a non-interacting Fermi gas, the band structure of a periodic lattice is independent of individual electron motions.

When a weak interaction is assumed amongst the particles, the system immediately becomes a lot more complex. Weak interactions amongst the particles can often be projected onto an effective interaction of one particle with the entire ensemble. Hence, the interaction term causes an additional static potential term, known as mean field [27, 43, 44]. The result is a single-particle description, similar to the non-interacting case, but the effective potential takes the interaction terms into account. Systems which can be described using this perspective are referred to as weakly correlated. Prominent examples are properties of ordinary metals. In fact, this picture of weak correlations captures the complete temperature-dependent behavior of transport properties, such as the specific heat capacity, the electrical resistivity and the magnetic susceptibility.

In contrast, there are plenty of phenomena which cannot be explained assuming weak interactions. To make matters worse, some phenomena only emerge due to a strong interaction amongst all the particles, allowing the entire ensemble to form a so-called correlated ground state. Generally, these strongly correlated states can no longer be depicted by a single particle in an effective field. The Thomas-Fermi approach is one ab-initio method to incorporate collective phenomena of solids to predict their macroscopic properties. A complimentary ansatz to understand many-body systems was introduced by Landau's ,quasiparticle' concept in 1957 [23. 24 and is called Landau Fermi-liquid theory.

Landau Fermi-liquid theory led to the phenomenological description of many phenomena, for example the electrical resistivity or the optical response of heavy fermions [19]. The physical quantities of Fermi liquids behave qualitatively very similar to Fermi gases, however sometimes their behavior has to be renormalized 45 .

Some systems show a ,strange behavior that cannot be understood in the framework of the phenomenological Fermi-liquid theory. They are often referred to as non-Fermi liquids or ,strange metals' 46 48. The most basic case of non-Fermi liquids are one-dimensional metallic systems. The Fermi-liquid description of

${ }^{1}$ The differential equations describing the motion of particles in the phase space are called equations of motion. 
these systems results in the Peierls instability [49]. Therefore, the so-called Luttinger-liquid description is more appropriate to describe this non-Fermi-liquid behavior, which is caused by the reduced dimensionality [50,52]. Other examples of systems with non-Fermi-liquid behavior are systems close to QPTs as they can be found in heavy-fermion materials [13, 53, 54].

\subsection{The Fermi-gas approach towards weak correlation}

In weakly correlated systems, the interaction amongst the particles can often be treated as a perturbation. A conventional metal consists of a rigid lattice of positively charged ions and mobile, negatively charged electrons. It turns out that the mobile conduction electrons are the main contributors for most transport properties [38, 39]. A common way of describing matter in condensed matter physics is the Hamilton formalism. It describes how the particles are evolving in the phase space, while taking the interaction as well as the kinetic energy of the particles into account. Finding the eigenstates of the Hamilton operator is in general a challenging task. In this section, the eigenstates for a system consisting of non-interacting fermions are described. Because of the negligible interactions amongst the particles, such a system is called a free Fermi gas. However, the interactions are subsequently introduced in a perturbative manner. The following Hamiltonian describes a weakly interacting Fermi gas, where the ionic lattice is treated as a homogeneously charged background:

$$
\mathcal{H}_{\text {Fermi gas }}=\underbrace{\sum_{i} \frac{\hbar^{2} \vec{k}_{i}^{2}}{2 m}}_{\text {kin. energy }}+\underbrace{\frac{1}{2} \sum_{i \neq j} \frac{e^{2}}{\left|r_{i}-r_{j}\right|}}_{\text {Coulomb repulsion }}
$$

where $i$ and $j$ subscript the electrons, $\vec{k}_{i}$ is the momentum vector of the $i$-th electron, $m$ the bare electron mass and $e$ the electric charge of an electron. At first, only the kinetic energy term is discussed. The Coulomb repulsion term can later be taken into account in a perturbative manner.

\subsubsection{The non-interacting Fermi gas}

By neglecting the Coulomb repulsion between electrons, the system is simplified to $N$ non-interacting electrons. A periodic lattice with lattice constant $a$ is assumed. The electronic states can be described using plane waves:

$$
\phi_{\vec{k}}=\frac{1}{\sqrt{\Omega}} e^{i\left(k_{x} x+k_{y} y+k_{z} z\right)}=\frac{1}{\sqrt{\Omega}} e^{i \vec{k} \vec{r}}
$$


where $\vec{k}$ is the indexing vector which corresponds to the momentum vector of the plane wave. The volume of the electron gas is labeled as $\Omega$ and $\vec{r}$ depicts the spatial coordinates. If periodic boundary conditions are assumed, the momentum vector components are given by the following equation:

$$
k_{i}=\frac{2 \pi}{\sqrt[3]{\Omega}} n_{i}, i \in x, y, z
$$

where $n_{i}$ are integers. Therefore, the electric states of a periodic lattice are represented in the momentum space (which is mathematically equivalent to the Fourier space) by a periodic lattice with lattice constant $\frac{2 \pi}{\sqrt[3]{\Omega}}$. The energy for a given state with a wave vector $\vec{k}$ is given by the eigenvalue of the kinetic part of the Hamiltonian 2.1.

$$
E_{\vec{k}}=\frac{\hbar^{2} \vec{k}^{2}}{2 m}=\frac{\hbar^{2}}{2 m}\left(\frac{2 \pi}{\sqrt[3]{\Omega}}\right)^{2}\left(n_{x}^{2}+n_{y}^{2}+n_{z}^{2}\right)
$$

which are discrete points located on a paraboloid. The larger the volume $\Omega$, the denser the electronic states become in the momentum space.

a

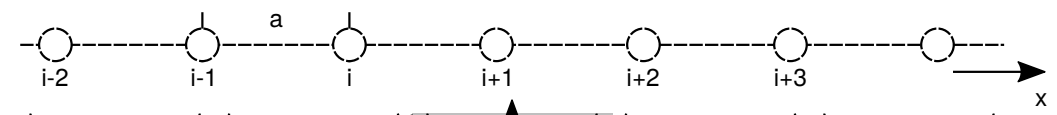

b

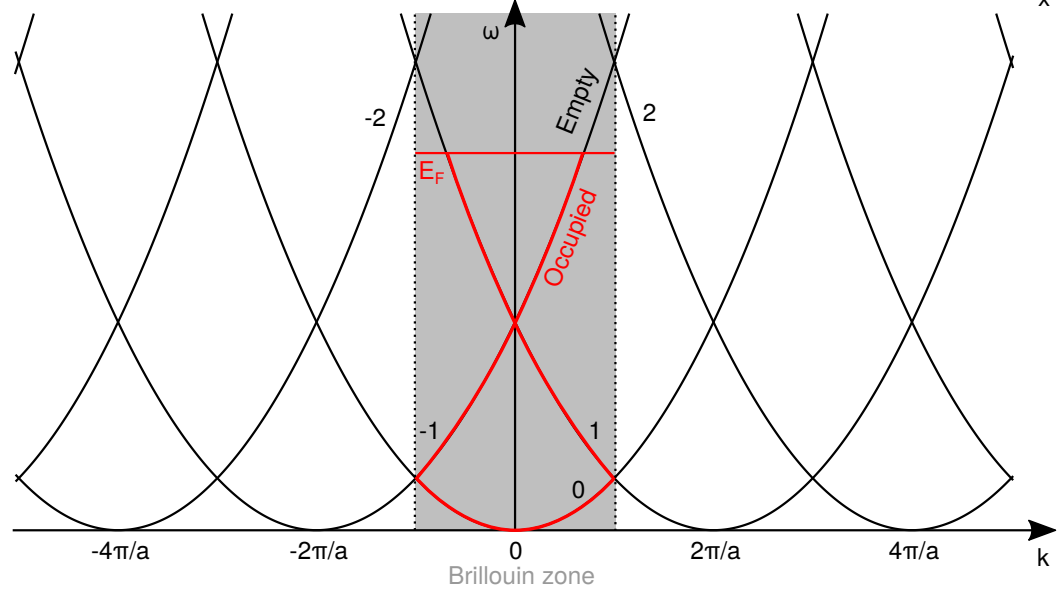

Figure 2.1.: (a) The electronic eigenstates of a one-dimensional chain of atoms with a lattice constant $a$ shall be described in the momentum space. (b) The eigenstates (at $0 \mathrm{~K})$ are described by a periodic arrangement of parabolas in the momentum space (see Eq. 2.4). Due to the periodicity, the band structure can be limited to the so-called Brillouin zone (gray shaded), without losing any information. At $T=0 \mathrm{~K}$, all eigenstates up to a certain energy level $E_{\mathrm{F}}$ are occupied (indicated by the red line). Above this energy threshold, the states remain empty. 
Eventually, they are assumed to be continuous as the volume $\Omega$ increases. Because of the aforementioned periodicity, the reciprocal space can be restricted to a minimum volume required to fully describe the entire lattice. The minimally required reciprocal space is called the Brillouin zone. The well-defined dispersion relation pinpoints the particle nature and the allowed states form the so-called band structure, which describes the mobile electrons in a solid. In a one-dimensional lattice with a single type of atoms, this minimal unit corresponds to the interval $k \in\left(-\frac{\pi}{a}, \frac{\pi}{a}\right)$ and leads to the band structure illustrated in Fig. 2.1.

According to the Pauli exclusion principle, each electronic state (indexed by $\vec{k}$ ) can be empty, partially filled with a single electron, or fully occupied with two electrons forming a spin-singlet state. To minimize the overall energy, the bands are filled bottom to top. The boundary between the highest occupied and the lowest unoccupied state defines the Fermi energy $E_{\mathrm{F}}$ and the corresponding Fermi wave vector $\vec{k}_{\mathrm{F}}$. In the case of a free electron gas in three dimensions, the Fermi surface is a sphere with radius $\left|\vec{k}_{\mathrm{F}}\right|$. In complex structures, the Fermi surface can have a complex shape, giving rise to some exquisite ground states such as for example spinor charge-density waves. These exquisite states are energetically favorable if great portions of the Fermi surface are well-nested ${ }^{2}$ [55]. The nesting properties of the Fermi surface play a crucial role in the conventional quantum critical scenario, see chapter 3. For the transport properties of metals, the number of electrons in close proximity of the Fermi surface plays a major role. Therefore, the energy-dependent density of states (DoS) $\rho(E)$ is defined, which specifies the number of electronic states in the energy interval $\mathrm{d} E$ around the energy $E$.

$$
\rho(E):=\frac{\mathrm{d} N(k)}{\mathrm{d} k} \frac{\mathrm{d} k}{\mathrm{~d} E}
$$

The DoS $\rho(E)$ furthermore depends on the dimensionality of the space. In case of a three-dimensional free electron gas, the DoS reads as follows:

$$
\rho_{3 \mathrm{D}}(E)=\frac{\mathrm{d} N(k)}{\mathrm{d} k} \frac{\mathrm{d} k}{\mathrm{~d} E}=\frac{2 \Omega}{(2 \pi)^{3}} 4 \pi k^{2} \frac{m}{\hbar^{2} k}=\frac{\Omega m}{\pi^{2} \hbar^{3}} \sqrt{2 m E} \propto \sqrt{E} .
$$

In weakly correlated conventional metals the Fermi temperature $T_{\mathrm{F}}$ is of the order of $10^{4} \mathrm{~K}$, see table 2.1 .

\begin{tabular}{c|c|c|c} 
Element & Fermi temp. $(\mathrm{K})$ & Fermi velocity $(\mathrm{m} / \mathrm{s})$ & Fermi vector $(\mathrm{kg} \mathrm{m} / \mathrm{s})$ \\
\hline $\mathrm{Cu}$ & $8.16 \cdot 10^{4}$ & $1.57 \cdot 10^{6}$ & $1.43 \cdot 10^{-24}$ \\
$\mathrm{Au}$ & $6.42 \cdot 10^{4}$ & $1.4 \cdot 10^{6}$ & $1.28 \cdot 10^{-24}$
\end{tabular}

Table 2.1.: Typical Fermi-surface values for ordinary metals.

\footnotetext{
${ }^{2} \mathrm{~A}$ Fermi surface is well-nested if great portions of the Fermi surface are parallel. These sections can be spanned by a constant vector $\vec{q}$, which is called the nesting vector. Chromium is a famous example which has a well-nested Fermi surface.
} 


\subsubsection{Perturbative treatment of Coulomb interaction in a Fermi gas}

Often, the interaction amongst the electrons are the driving force of the observed phenomena and, hence, the free Fermi-gas model has to be modified. However, discussing the Hamiltonian $\mathcal{H}_{\text {Fermi gas }}$ turns quickly into a complex debate. Since the 1950s, many theoreticians were working on the many-body problem and trying to find an appropriate description for interacting systems. One possibility is to take the Coulomb repulsion term as an ab-initio perturbation ${ }^{3}$ into account, see e.g. [40].

Treating the Coulomb repulsion term in a perturbational manner leads to a renormalized energy of the ground state with respect to the free electron gas described in section 2.2.1. By treating the Coulomb repulsion as a perturbation, the ground state is described by an additional energy term. This additional energy shift is also called the self-energy of the system [40]. The physical meaning of the self-energy can be understood as follows: The self-energy describes the effective potential felt by a single electron due to Coulomb repulsion with the other electrons. Hence, every electron modifies its surrounding and therefore increases the systems' overall energy.

The perturbational ansatz of treating the Coulomb interaction is expected to become problematic, as soon as the interaction amongst the fermions is no longer small. Nevertheless, many systems with a strong interaction amongst the fermions still behave like a Fermi gas at sufficiently low temperatures. In 1957, Landau gave a phenomenological explanation for this remarkable observation [23, 24, as it will be discussed in section 2.3 .

\subsection{Fermi-liquid theory}

Landau explained phenomenologically why strongly interacting fermion systems can show a low-temperature behavior which is similar to a Fermi gas. He assumed that an interacting fermion system can be described by so-called ,quasiparticles', which only weakly interact amongst each other 4 [55. Landau's theory is nowadays known as Fermi-liquid theory. Because of the interaction term, these quasiparticles (i.e. the excited states of the system) have a finite lifetime, see section 2.6.

One characteristic difference between a Fermi liquid and a Fermi gas can be found in the occupation number at $0 \mathrm{~K}$. In a Fermi gas, the occupation number is described by the Fermi-Dirac distribution, which abruptly jumps from ,fully occupied' to ,empty ${ }^{6}$ at the Fermi surface for $T=0 \mathrm{~K}$ [55]. However, for a Fermi liquid the

\footnotetext{
${ }^{3} \mathrm{~A}$ well-known ab-initio perturbational approach is the Hartree-Fock approximation [43, 44]. In the Hartree-Fock approximation, the interaction amongst the same particles is treated as a mean field, allowing to estimate the wave functions as well as the energy of the system.

${ }^{4}$ In the asymptotic limit (i.e. $k_{\mathrm{B}} T \ll E_{\mathrm{F}}$ ), they can be even regarded as free fermions with an effective mass $m^{*}$.
} 
occupation number starts to decrease below the Fermi surface and is not zero above the Fermi surface, even at $T=0 \mathrm{~K}$. The reason is the interaction amongst the fermions, which is always present. Consequently, the ,jump' of the occupation number at the Fermi surface is 1 for a Fermi gas, whereas the ,jump' for a Fermi liquid is reduced to a value $Z$ which is smaller than $1 . Z$ is called the quasiparticle residue [55, 56]. The quasiparticle residue $Z$ is a direct consequence of the existence of quasiparticles, see also section 2.6 .

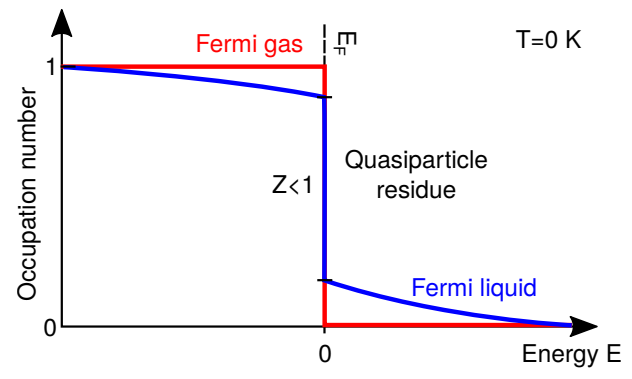

Figure 2.2: The difference between a free Fermi gas and a Fermi liquid manifests in occupation behavior at $T=0 \mathrm{~K}$. The Fermi gas is described by the Fermi-Dirac distribution, which jumps between 1 and 0 at $E_{\mathrm{F}}$ (red line). For a Fermi liquid, this jump at the Fermi surface is reduced to $Z<$ 1 (blue curve). The jump height defines the quasiparticle residue $Z<1$ of a Fermi liquid.

However, this Fermi-liquid picture is only valid for sufficiently low temperatures as only then the system is close enough to its ground state. The temperature range, in which the quasiparticle picture is applicable, is strongly depending on the system itself.

As the ground state of an ordinary metal is a fully occupied Fermi sea, the thermal fluctuations should only excite a limited number of electrons above the Fermi energy. This criterion is fulfilled for temperatures well below the Fermi temperature $T_{\mathrm{F}}$, which for typical metals is in the order of $10^{4} \mathrm{~K}$, see also Table 2.1 . Hence, Fermi-liquid behavior for normal metals is already expected at room temperature.

A heavy-fermion Fermi-liquid behavior on the other hand can only establish once the heavy band has formed. The heavy band (which is caused by a spectral weight shift, see section 2.5.1) is a direct consequence of the strong correlation, which only sets in at sufficiently low temperatures. In this case, the energy scale is given by the Kondo temperature $T_{\mathrm{K}}$. In order to describe the observed behavior with Fermi-liquid theory, the response has to be strongly renormalized. This renormalization can be interpreted as an increase of the effective electron mass $m^{*}$, as this is the only degree of freedom in Fermi-liquid theory. This increase of the effective electron mass $m^{*}$ explains the term ,heavy fermion'. 


\subsection{The Kondo-impurity effect}

The behavior of a magnetic impurity in a metallic environment is captured by

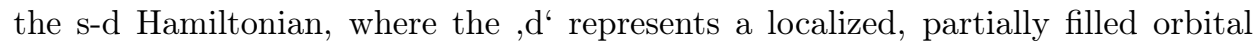
and, $\mathrm{s}^{6}$ the spatially extended, delocalized conduction orbitals. Therefore, the s-d Hamiltonian describes two effects: the Kondo screening of a single magnetic moment as well as the spin polarization induced into the conduction electrons in close proximity of the magnetic moment [40. This second effect can ultimately lead to macroscopic magnetic ordering, if the magnetic impurities are arranged on a sufficiently dense periodic lattice, see section 2.5. However, the s-d Hamiltonian only describes the Kondo-impurity case.

\subsubsection{Induced spin-polarization in conduction electrons}

The following Hamiltonian describes the system of a single magnetic impurity embedded into a sea of conduction electrons, while neglecting the interaction amongst the conduction electrons themselves [39]:

$$
\begin{aligned}
\mathcal{H}_{\text {impurity }}= & \mathcal{H}_{0}+\mathcal{H}_{\mathrm{s}-\mathrm{d}} \\
= & \sum_{\vec{k}, \sigma} E_{\vec{k}} c_{\vec{k}, \sigma}^{\dagger} c_{\vec{k}, \sigma}+\frac{J}{2 N} \sum_{\vec{k}_{1}, \vec{k}_{2}}\left(c_{\vec{k}_{2}, \uparrow}^{\dagger} c_{\vec{k}_{1}, \uparrow}-c_{\vec{k}_{2}, \downarrow}^{\dagger} c_{\vec{k}_{1}, \downarrow}\right) S_{z} \\
& -c_{\vec{k}_{2}, \downarrow}^{\dagger} c_{\vec{k}_{1}, \uparrow} S_{+}-c_{\vec{k}_{2}, \uparrow}^{\dagger} c_{\vec{k}_{1}, \downarrow} S_{-},
\end{aligned}
$$

where $\mathcal{H}_{0}$ describes the conduction band and $\mathcal{H}_{\mathrm{s}-\mathrm{d}}$ characterizes the exchange coupling between the magnetic impurity $S_{z}$ and the conduction electrons. $c_{\vec{k}, \sigma}^{\dagger}$ $\left(c_{\vec{k}, \sigma}\right)$ are the creation (annihilation) operators of conduction electrons with momentum $\vec{k}$ and spin $\sigma$, while the $S_{+}\left(S_{-}\right)$operator increases (decreases) the spin state of the impurity. Assuming that the localized orbital is filled by a single electron, $S_{\mathrm{z}}$ can only take the two values: $\pm 1 / 2$. Furthermore, $S_{+}$and $S_{-}$can be expressed by the Pauli matrices $\sigma_{x, y}$ [40]:

$$
\begin{aligned}
& S_{+}=\left(\begin{array}{ll}
0 & 1 \\
0 & 0
\end{array}\right)=\frac{1}{2}\left[\sigma_{x}+i \sigma_{y}\right]=\frac{1}{2}\left[\left(\begin{array}{ll}
0 & 1 \\
1 & 0
\end{array}\right)+i\left(\begin{array}{cc}
0 & -i \\
i & 0
\end{array}\right)\right], \\
& S_{-}=\left(\begin{array}{ll}
0 & 0 \\
1 & 0
\end{array}\right)=\frac{1}{2}\left[\sigma_{x}-i \sigma_{y}\right]=\frac{1}{2}\left[\left(\begin{array}{ll}
0 & 1 \\
1 & 0
\end{array}\right)-i\left(\begin{array}{cc}
0 & -i \\
i & 0
\end{array}\right)\right] .
\end{aligned}
$$

$N$ labels the number of atomic sites and $J$ is the coupling constant, which is typically positive for rare-earth compounds. The temperature-dependent, but strictly positive coupling constant $J$ leads to an antiparallel arrangement of the magnetic moments at sufficiently low temperatures. The $\mathcal{H}_{\mathrm{s}-\mathrm{d}}$ Hamiltonian has four different terms which can be understood as the following scattering processes: The first two describe the scattering process without any spin-flip of the conduction 
electron and the second two terms describe scatting with an additional spin exchange. These four possible scattering cases are illustrated in Fig. 2.3. The sign in the Hamiltonian indicates whether the force is attractive or repulsive. If a spin-up conduction electron scatters with the magnetic impurity with a parallel magnetic moment, the conduction electron is being repelled as the two magnetic moments form a triplet state, see Fig. 2.3 (a). In the case of a spin-down electron, the conduction electron and the magnetic impurity form a singlet state and the electron is attracted, see Fig. 2.3 (b). As the conduction electron passes the magnetic impurity, also magnetic moment between the conduction electron and the impurity can be exchanged. Hence, the impurity as well as the conduction electron have a different magnetic configuration before $\left(t=t_{1}\right)$ and after $\left(t=t_{2}\right)$ the Kondo scattering, see Fig. 2.3 (c,d).
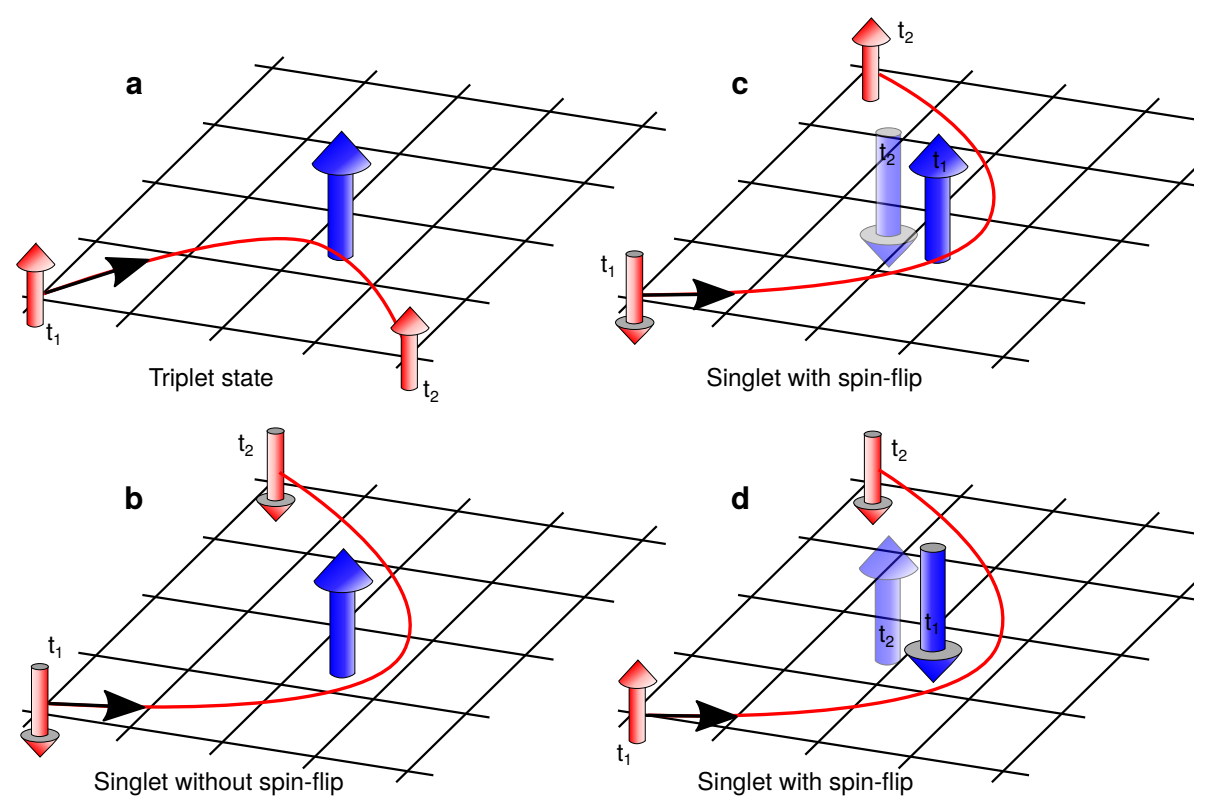

Figure 2.3.: The Kondo scattering can illustrated as follows, where $t_{1}$ describes the situation before the scattering process and $t_{2}$ the final situation: (a) A spin-up conduction electron (red) approaches a magnetic impurity (blue). Because the two spins are parallel (triplet state), the conduction electron is repelled. (b) A spin-down conduction electron approaches a magnetic impurity $\left(t=t_{1}\right)$. Because the two spins are antiparallel (singlet state), the conduction electron is deflected. The spins of the magnetic impurity and the conduction electron remains unaffected throughout the scattering process. (c,d) The same scenario as described in (b), but with an additional spin exchange. The conduction electron as well as the magnetic impurity have a different spin polarization before $\left(t=t_{1}\right)$ and after $\left(t=t_{2}\right)$ the scattering process. 
The upper discussion shows that the magnetization of the impurity is fluctuating as the magnetic moment can be transferred between the conduction electrons and the localized magnetic impurity. This locally leads to a ,quenching' of the magnetic moment, which is called Kondo screening. As the local magnetic moment is affected by the magnetic moment of the conduction electrons, in return also the conduction electrons get spin polarized by an impurity with (fixed) magnetization $\langle S\rangle$, as they feel an effective magnetic field $H_{\mathrm{q}}$ [39]:

$$
H_{\mathrm{q}}=\frac{\frac{J}{N}\langle S\rangle}{\frac{1}{2} g \mu_{\mathrm{B}}}
$$

where $g$ is the gyromagnetic ratio and $\mu_{\mathrm{B}}$ is the Bohr magneton. Hence, the corresponding spin polarization $\sigma_{\mathrm{RKY}}$ of the conduction electrons reads as 39, 40]:

$$
\sigma_{\mathrm{RKY}}(r) \propto \frac{-2 k_{\mathrm{F}} r \cos 2 k_{\mathrm{F}} r+\sin 2 k_{\mathrm{F}} r}{\left(2 k_{\mathrm{F}} r\right)^{4}}
$$

Eq. 2.11illustrates the induced spin polarization of the conduction electrons, which is strongly damped with increasing distance from the impurity. Furthermore, it oscillates with twice the frequency specified by the Fermi vector $k_{\mathrm{F}}$. The spin-polarization described in Eq. 2.11 is called Ruderman-Kittel-Yosida (RKY) oscillation.

So far, only the effect of a magnetic impurity on the conduction electrons was considered. However, if a second magnetic impurity is introduced at a distance $R$ from the original magnetic impurity, the magnetic moments of the two impurities are coupled via the spin-polarization of conduction electrons. This coupling is described by the Ruderman-Kittel-Kasuya-Yosida (RKKY) interaction term [57, 58]:

$$
\mathcal{H}_{\mathrm{RKKY}} \propto \frac{-2 k_{\mathrm{F}} R \cos 2 k_{\mathrm{F}} R+\sin 2 k_{\mathrm{F}} R}{\left(2 k_{\mathrm{F}} R\right)^{4}} \vec{S}_{1} \cdot \vec{S}_{2}=: J_{\mathrm{RKKY}} \vec{S}_{1} \cdot \vec{S}_{2}
$$

If the impurities are located on a dense periodic lattice, the RKKY interaction causes a magnetic ordering via the spin channels of the conduction electrons. The RKKY interaction plays an important role in realizing magnetic instabilities as they occur in QPTs, see chapter 3

\subsubsection{The Kondo effect in dilute alloys}

In an ordinary metal, the temperature-dependent electrical resistivity can be calculated using the first Born approximation, which takes first-order perturbations into account. Kondo additionally discussed the second-order perturbation terms, which allowed him to explain the Kondo effect [17. His solution led to a minimum 
in the electrical resistivity, but simultaneously to a divergence at $0 \mathrm{~K}$, which is physically not plausible. However, taking even higher-order perturbation terms into account, this divergence at $0 \mathrm{~K}$ again disappears. A detailed discussion can be found in various textbooks, e.g. in [38, 40, 42].

Descriptive derivation The $\mathcal{H}_{\mathrm{s}-\mathrm{d}}$ Hamiltonian describes the interaction between free conduction electrons and a single localized magnetic moment. The electrical conductivity $\sigma$ can be described using the Fermi velocity of the electron $v_{\vec{k}}$ and a mean-free scattering time $\tau$ [40, 42]:

$$
\sigma=-\frac{2 e^{2}}{3 \Omega} \int \tau v_{\vec{k}}^{2} \rho\left(E_{\vec{k}}\right) \frac{\partial f}{\partial E_{\vec{k}}} \mathrm{~d} E_{\vec{k}}
$$

where $\rho\left(E_{\vec{k}}\right)$ is the DoS and $f$ is the Fermi-Dirac distribution. Hence, the electrical conductivity is proportional to the squared Fermi velocity as well as to the DoS. The expression $\frac{\partial f}{\partial E_{\vec{k}}}$ ensures that only the electrons in close vicinity of the Fermi surface $\left(E_{\mathrm{F}} \pm k_{\mathrm{B}} T\right)$ contribute. The scattering mechanisms can be described by a single the constant $\tau$, which is calculated using Fermi's golden rule [40, 41, 59, 61]:

$$
\frac{1}{\tau}=\frac{2 \pi}{\hbar} \sum_{f}\left|\left\langle\vec{k}_{2}, \sigma_{2}|T(E)| \vec{k}_{1}, \sigma_{1}\right\rangle\right|^{2} \delta\left(E-E_{\vec{k}_{2}}+E_{\vec{k}_{1}}\right)
$$

where $T(E)$ is the transition matrix element and the delta function $\delta$ guarantees for the energy conservation. Hence, the Kondo effect emerges due to higher order terms of the transition matrix element. In order to calculate the conductivity described by Eq. 2.13 the transition matrix $T$ is Taylor expanded. The first-order term expansion $T^{(1)}$ leads to the electrical resistivity found by Born [40, 62]:

$$
R_{\mathrm{Born}}=\frac{3}{2} \frac{m \pi}{e^{2} \hbar} \frac{\Omega}{E_{\mathrm{F}}}\left(\frac{J}{2 N}\right)^{2} S(S+1)
$$

where $S$ is the localized spin. The Born approximation is a first-order approximation as multiple scattering processes are neglected. This approximation is only reasonable if the energy of the incident particle is much larger than the scattering potential. Therefore, the Born approximation is valid at high temperatures. It becomes apparent that the Born approximation is not valid in the Kondo screening regime, since the conduction electrons are strongly affected by the magnetic scattering centers [63]. The resistivity minimum and therefore the Kondo effect can only be found by taking the second- (or even higher-) order terms of the transition matrix element $T$ into account. The first- and second-order terms together lead to the following electrical resistivity [17, 64]:

$$
R_{\text {Kondo }}=R_{\text {Born }}\left[1+\frac{2 J \rho}{N} \ln \frac{k_{\mathrm{B}} T}{W}\right] .
$$


For the analytical derivation of Eq. 2.16, the conduction band is assumed to be between $-W$ and $+W$ and the Fermi surface is set to zero [40. For simplicity, the DoS of the conduction band is assumed to be constant:

$$
\begin{aligned}
& \rho(E)=\rho=\text { const., for }-W<E<W \text { and } \\
& \rho(E)=0, \text { for } E<-W \text { or } E>W .
\end{aligned}
$$

At high temperatures, the Kondo resistivity $R_{\text {Kondo in Eq. } 2.16 \text { predicts }}$ qualitatively the same temperature-dependent resistivity as calculated in the Born approximation, see Eq. 2.15. However, as the temperature scale becomes comparable to the band width, the sign of the second term changes. Hence, a logarithmic divergence in the resistivity emerges, see Fig. 2.4. For temperatures well below the band width $2 W$, the logarithmic divergence dominates. The relevant temperature scale, below which the logarithmic divergence sets in, defines the Kondo temperature $T_{\mathrm{K}}$ 40]:

$$
T_{\mathrm{K}}:=\frac{W}{k_{\mathrm{B}}} e^{\frac{-N}{|J \rho|}}
$$

With this definition of $T_{\mathrm{K}}$ the temperature-dependent electrical resistivity can be rewritten as:

$$
R_{\text {Kondo }}=R_{\text {Born }}\left[1+\frac{\log k_{\mathrm{B}} T-\log W}{\log k_{\mathrm{B}} T_{\mathrm{K}}-\log W}\right] .
$$

In a descriptive picture, the Kondo temperature describes energy scale at which the magnetic impurity moments are fully screened by the conduction electrons. In fact, because these ,dressed' magnetic impurities have greatly enhanced scattering cross section compared to non-magnetic impurities, the electrical resistivity increases as the system is further cooled down [30].

The explanation of the increasing electrical resistivity with decreasing temperature is one of the earliest theoretical explanations of a transport phenomenon caused by strong correlation 5 However, the electrical resistivity found by Kondo diverges in the limit of $T \rightarrow 0 \mathrm{~K}$, which is unphysical. This divergence is an artifact of the second-order approximation as it can be suppressed by taking higher-order terms into account, as demonstrated by Abrikosov [66].

The formation of Kondo singlets has a profound effect on the local spectral weight. At temperatures well above the Kondo temperature $T_{\mathrm{K}}$, the Kondo screening is strongly reduced. Hence, the entire local spectral weight of the orbital is located at the orbital energy, which is far below the Fermi energy $E_{\mathrm{F}}$. However, at temperatures comparable to $T_{\mathrm{K}}$, the magnetic orbital starts to form Kondo singlets with its surrounding conduction electrons. This resonance scattering process causes

\footnotetext{
${ }^{5}$ BCS theory for conventional superconductivity was the first theory, which explained a strongly correlated transport phenomenon 65.
} 


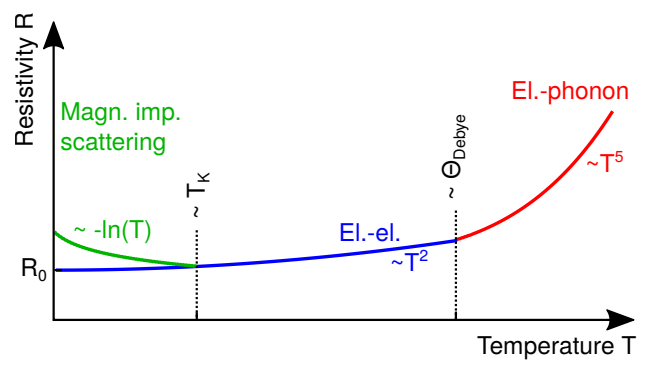

Figure 2.4.: Illustration of the effect of magnetic impurities on the temperature-dependent electrical resistivity. In an ordinary metal, the resistivity at high temperatures $\left(T>\Theta_{\text {Debye }}\right)$ is governed by electron-phonon scattering, which leads to a $T^{5}$-dependent resistivity. Below this temperature, the electron-electron scattering is dominant, leading to a $T^{2}$ dependence of the resistivity (Fermi-liquid behavior). Magnetic impurities dominate the resistivity behavior only below the characteristic Kondo temperature $T_{\mathrm{K}}$, causing a logarithmic divergence. For more details, see text.

a small fraction of the spectral weight to be locally transferred to the Fermi surface. This shifted spectral weight has a Lorentz peak-like structure, which is characteristic for resonance scattering. This tiny fraction of spectral weight accumulated for each impurity site at the Fermi surface is called Kondo spectral weight. Because the Kondo spectral weight resonance is very sharp, the lifetime is extremely long, as it is almost an eigenstate of the system ${ }^{6}$ The width of the Kondo resonance at the Fermi surface defines an effective Kondo temperature $T_{\mathrm{K}}^{*}$. Hence, the lifetime of the Kondo state is governed by this inverse effective Kondo temperature $T_{\mathrm{K}}^{*}$. More details about the Kondo resonance and its lifetime are given in the sections 2.5 and 2.6 .

\subsection{The Anderson Hamiltonian}

If the magnetic impurities are arranged on a dense, periodic lattice throughout the carrier metal, the RKKY interaction has to be taken into account, see section 2.4.1. This additional effect ultimately leads to the thrilling field of quantum criticality and non-Fermi-liquid behavior, which cannot be observed in dilute magnetic alloys.

In 1961 Anderson proposed a Hamiltonian describing a magnetic sublattice embedded in a metallic environment. If the RKKY interaction is dominant, the system orders magnetically, which means that the magnetic moments are not fully screened. In this case, no heavy-fermion behavior is observed. On the other hand, if the magnetic moments are fully Kondo screened, the system is in a heavy-fermion

${ }^{6}$ Eigenstates have an infinitely sharp energy level since the width of the state is inversely proportional to the lifetime of the state. 
state and does not show any macroscopic magnetic ordering, see section 2.5.1. A metallic system with a magnetic sublattice is described by the so-called periodic Anderson Hamiltonian, which is an on-site description of the interaction between magnetic impurities and conduction electrons [39, 40].

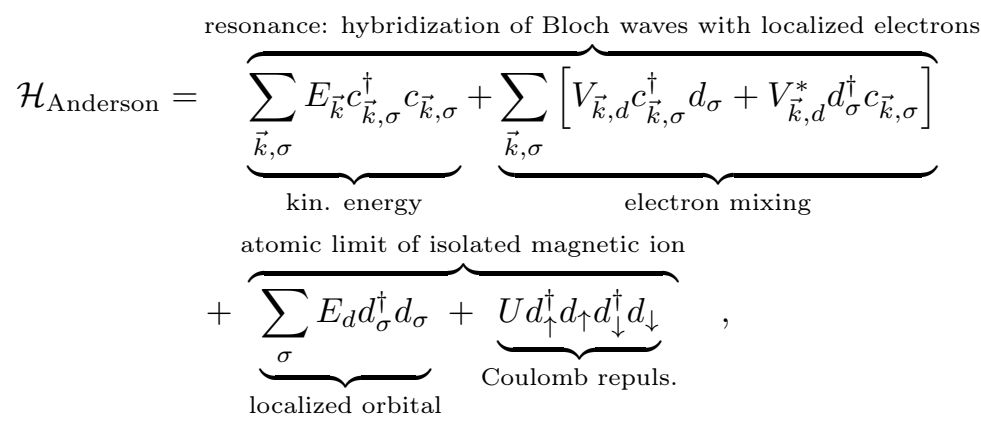

where the first term describes the dispersion relation of the conduction electrons, analogously to the Kondo-impurity case, see Eq. 2.7. The second term expresses the mixed electron state between the localized magnetic moment $d_{\sigma}$ and the conduction electrons, where either an electron is excited into the conduction band or absorbed into a localized orbital. These two processes are proportional to the electron-mixing integral $V_{\vec{k} d}$. It describes the resonant scattering between the delocalized conduction electrons (Bloch waves) and the localized electrons, as also found in the Kondo-impurity scenario. However, only in the case of a periodic arrangement a ,heavy' band forms and as a result an avoided crossing (i.e. a lifting of the degeneracy) at the Fermi surface can establish [30]. The last two terms of the Hamiltonian express the energies of the spatially confined orbital, where the penultimate describes the orbital and the last term the Coulomb repulsion in the case of double occupancy. Hence, the Anderson Hamiltonian fully describes the Kondo-lattice problem. In the following section, the appearance of a resonance at the Fermi surface is discussed.

\subsubsection{The Kondo resonance emerging at the Fermi surface}

One of the most prominent features of the Kondo effect is the massive increase of the DoS at the Fermi surface. With a simplified Anderson Hamiltonian, where the Coulomb repulsion $U$ in the localized orbital term is neglected, the huge DoS can be understood. From the discussion of the Kondo impurity, see section 2.4.2, it is known that locally spectral weight is transferred to the Fermi surface. In a lattice configuration, this corresponds to a very shallow Kondo band. The electron-mixing term in Eq.2.20 ultimately leads to an avoided crossing between the conduction band and the shallow Kondo band at the Fermi surface, causing a large increase in the DoS. Because its origin is resonance scattering, a Lorentz-shaped resonance is expected. The width of the resonance peak is proportional to the 
squared electron-mixing integral $V_{\vec{k}, d}$, which for simplicity is here assumed to be independent of $\vec{k}$ and the spin $\sigma$. Hence, it can be labeled as $V$. The electron-mixing integral is furthermore also in the range of the Kondo temperature $T_{\mathrm{K}}$, which was introduced in section 2.4 .2 for the impurity case:

$$
\Delta \propto|V|^{2} \int \rho_{c}(E) \delta\left(E_{\mathrm{d}}-E\right) \mathrm{d} E=|V|^{2} \rho_{\mathrm{c}}\left(E_{\mathrm{d}}\right)
$$

where $\rho_{\mathrm{c}}$ is the DoS of conduction electrons. $\Delta$ is the half width at half maximum of the Lorentzian-shaped resonance at the Fermi energy. The hybridization gap depends quadratically on the electron-mixing integral $V$ and linearly on the DoS at $E_{\mathrm{d}}$. The resonance width of an excited state scales inversely with the lifetime of the excitation, see section 2.6 .

As the physical properties are mainly depending on the electrons in the vicinity of the Fermi surface, see chapter 4 , knowing the location of the chemical potential $\mu$ is crucial $~^{7}$ The chemical potential $\mu$ is defined as the threshold energy, at which exactly $1 / 2$ of all available states are occupied. Therefore, the chemical potential $\mu$ can be calculated as follows [40]:

$$
n_{\mathrm{d} \sigma}=\int_{-\infty}^{\mu} \rho_{\mathrm{d}}(E) \mathrm{d} E=\int_{-\infty}^{\mu} \frac{\Delta / \pi}{\left(E-E_{\mathrm{d}}\right)^{2}+\Delta^{2}} \mathrm{~d} E=\frac{1}{2}+\frac{1}{\pi} \arctan \frac{\mu-E_{\mathrm{d}}}{\Delta},
$$

where $\rho_{\mathrm{d}}$ corresponds to the DoS of the localized magnetic moments. If the chemical potential $\mu$ is at the hybridization energy $\left(\mu=E_{\mathrm{d}}\right)$, the occupation number $n_{\mathrm{d} \sigma}$ is exactly $1 / 2$. Thus, the resonance appears at the Fermi surface.

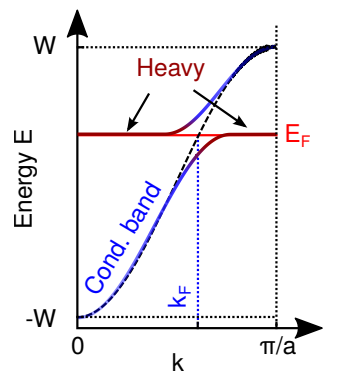

Figure 2.5: The Kondo effect causes locally a spectral weight accumulation of the localized orbitals at the Fermi surface. In the case of a dense lattice, this spectral weight forms a shallow band at the Fermi energy. In general, this band hybridizes with the conduction band (dashed line). The resulting band structure has shallow parts with a large effective mass $m^{*}$ (reddish part). Far from the Fermi surface, the conduction band remains unaffected (blue part).

\footnotetext{
${ }^{7}$ Strictly speaking, the Fermi energy $E_{\mathrm{F}}$ is only defined at $T=0 \mathrm{~K}$. For $T>0 \mathrm{~K}$, the chemical potential $\mu$ is defined as written in the text. At $T=0 \mathrm{~K}, \mu$ equals $E_{\mathrm{F}}$.
} 


\subsection{Quasiparticle lifetimes and spectral weight}

As described in section 2.3. Landau Fermi-liquid theory approaches strong correlations by introducing quasiparticles as excitations of a complex ground state. The lifetime of the quasiparticles can be illustrated as follows: A completely filled Fermi sea is assumed at $0 \mathrm{~K}$, which means each electron state with momentum $|\vec{k}| \leq k_{\mathrm{F}}$ is occupied. Thus, an additional electron with spin $\sigma_{1}$ can only be injected into the system if its energy is larger than the Fermi energy. As this single electron will interact with the Fermi sea, scattering processes with other electrons have to be considered. However, the phase-space volume greatly reduces if the excess energy of the injected electron is small $\left(E-E_{\mathrm{F}} \approx 0\right)$ [1]:

$$
\mathcal{H}_{\text {el. }- \text { el. }}=\sum_{\vec{k}_{1}, \vec{k}_{2}, \vec{q}, \sigma_{1}, \sigma_{2}} V(\vec{q}) c_{\vec{k}_{1}-\vec{q}, \sigma_{1}}^{\dagger} c_{\vec{k}_{2}+\vec{q}, \sigma_{2}}^{\dagger} c_{\vec{k}_{2}, \sigma_{2}} c_{\vec{k}_{1}, \sigma_{1}}
$$

where the $c_{\vec{k}, \sigma}^{\dagger}\left(c_{\vec{k}, \sigma}\right)$ are the creation (annihilation) operator for the $|\vec{k}, \sigma\rangle$ electronic state and $\vec{q}$ labels the momentum transfer between two electrons. $V(\vec{q})$ describes the short-ranged Yukawa potential. The electron-electron scattering processes are limited by three criteria: energy and momentum conservation as well as the Pauli principle. The lifetime can be calculated by Fermi's golden rule [41, 55, 59, 61]:

$$
\begin{array}{r}
\frac{1}{\tau_{\vec{k}}}:=\frac{1}{\tau_{\vec{k}_{1}}}=\frac{2 \pi}{\hbar \Omega^{2}} \sum_{\vec{k}_{2}, \vec{q}, \sigma_{2}}|V(\vec{q})|^{2} n_{\vec{k}_{2}}\left(1-n_{\vec{k}_{1}-\vec{q}}\right)\left(1-n_{\vec{k}_{2}+\vec{q}}\right) \\
\cdot \delta\left(E_{\vec{k}_{1}-\vec{q}}-E_{\vec{k}_{1}}-\left(E_{\vec{k}_{2}}-E_{\vec{k}_{2}+\vec{q}}\right)\right),
\end{array}
$$

where $n_{\vec{k}}$ is the occupation number of state $\vec{k}$. The $\delta$ function guarantees for the energy conservation whereas the first terms are required to fulfill the Pauli principle. Hence, the lifetime $\tau_{k}$ is inversely proportional to the excess energy squared, when assuming $T=0 \mathrm{~K}$ [41, 55]:

$$
\frac{1}{\tau_{\vec{k}}} \propto\left(E_{\vec{k}}-E_{\mathrm{F}}\right)^{2}
$$

Eq. 2.25illustrates that excited electrons which are close to the Fermi surface have a very long lifetime $\tau_{\vec{k}}$. The long lifetime is caused by the phase space available for the scattering processes. Due to energy conservation, the accessible phase space is limited to a shell of thickness $\left|E_{\vec{k}}-E_{\mathrm{F}}\right|$. Hence, the lifetime of an electron at the Fermi surface is infinite [55]. Furthermore, this result implicates that the spectral width of the resonance of the excited state is $\frac{\hbar}{\tau_{\vec{k}}}$. Hence, the coherent part of the 
excited states, i.e. the quasiparticles, can be illustrated in the momentum space as shown in Fig. 2.6

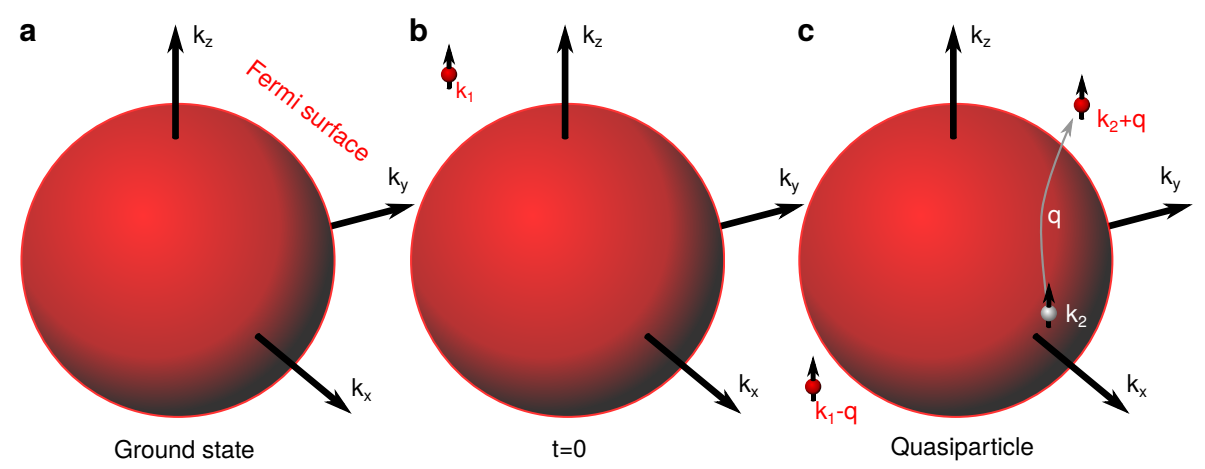

Figure 2.6.: (a) A fully occupied Fermi surface illustrates the unperturbed ground state. (b) At time $t=0$ an additional electron with momentum $k_{1}$ is introduced into the system at an energy $E>E_{\mathrm{F}}$. The introduced electron will interact with the electrons in the Fermi sea due to electron-electron scattering. (c) Due to a scattering process, the injected electron $k_{1}$ will release some of its energy and will eject a second electron $k_{2}$ out of the Fermi sea, creating a particle-hole excitation. Because the states $k_{2}+q$ and $k_{1}-q$ must be empty, the scattering rate is strongly reduced for $E \approx E_{\mathrm{F}}$. This behavior illustrates what is described by Eq. 2.25 . Adopted with permission from [41].

The spectral weight function $A(\vec{k}, \omega)$ of the resonance is given by the following expression [41]:

$$
A(\vec{k}, \omega) \propto \sum_{n}\left|\left\langle\psi_{n}\left|c_{\vec{k}, \sigma}\right| \psi_{0}\right\rangle\right|^{2} \delta\left(\hbar \omega-\hbar \omega_{n}\right)
$$

where $\left|\psi_{0}\right\rangle$ labels the ground state and $\left|\psi_{n}\right\rangle$ the excited state. The spectral weight function consists of a coherent, Lorentz-shaped part with a width which is inversely proportional to the lifetime of the excited state. Because the coherent part has a well-defined dispersion relation, the excitation has a ,particle-like' nature and can be considered to be a quasiparticle:

$$
A_{\text {coherent }}(\vec{k}, \omega)=\frac{Z_{\vec{k}} \hbar}{\pi \tau_{\vec{k}}} \frac{1}{\left(\hbar \omega-E_{\vec{k}}\right)^{2}+\left(\frac{\hbar}{\tau_{\vec{k}}}\right)^{2}}
$$

where $Z_{\vec{k}}$ is the quasiparticle residue, as introduced in section 2.3 . Because the quasiparticle residue $Z_{\vec{k}}$ is smaller than 1 , the remaining spectral weight has to be shifted towards higher energies, see Fig. 2.7. Since this remaining spectral weight does not have any dispersion relation, it is also called the incoherent part of spectral 
weight. Due to the lack of a dispersion relation, this part of the spectral weight is not ,particle-like ${ }^{6}$ and therefore no quasiparticle.

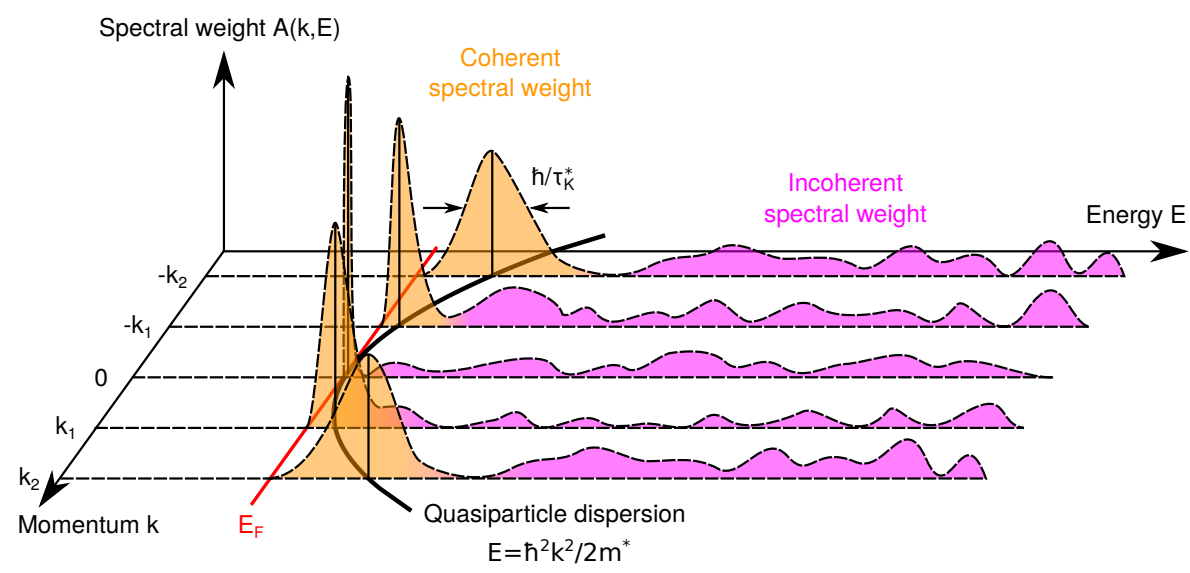

Figure 2.7.: Illustration of quasiparticle spectral weight $A(k, \omega)$ as a function of energy and momentum in a one-dimensional case. The quasiparticle residue $Z_{k}<$ 1 specifies the fraction of the spectral weight, which is captured in the coherent part (yellow). The coherent part of the spectral weight is a Lorentz-shaped curve (see Eq. 2.27), where the width is given by the inverse lifetime of the excited state. Because the coherent part has a well-defined dispersion relation (black line), the excitation can be considered to be a quasiparticle. The dispersion relation further defines the effective mass $m^{*}$ of the quasiparticles. Because the quasiparticle residue $Z_{k}$ is smaller than one, the missing spectral weight has to be shifted towards higher energies (purple). Because the remaining spectral weight does not show any well-defined dispersion relation, it is called the incoherent part of the spectral weight. Adopted with permission from [41]. 


\subsection{Crystal-field satellites and high-temperature Kondo effect}

Magnetic moments in atoms originate from partially filled shells, as uncompensated spins of the electrons are leading to a non-zero net magnetic moment. Partially filled shells are usually found in strongly confined orbitals. The most confined orbitals are the $\mathrm{d}$ and $\mathrm{f}$ shells. The energetically most favorable configuration is described by the three empirical Hund's rules [55]:

\begin{tabular}{c|c||c|c|c} 
element & shell & total $S$ & total $L$ & total $J$ \\
\hline $\mathrm{Fe}$ & $3 \mathrm{~d}^{6}$ & $4 \cdot 1 / 2$ & 2 & 4 \\
$\mathrm{Co}$ & $3 \mathrm{~d}^{7}$ & $3 \cdot 1 / 2$ & 3 & $9 / 2$ \\
$\mathrm{Ni}$ & $3 \mathrm{~d}^{8}$ & $2 \cdot 1 / 2$ & 3 & 4 \\
\hline $\mathrm{Ce}$ & $4 \mathrm{f}^{1}$ & $1 \cdot 1 / 2$ & 3 & $5 / 2$ \\
$\mathrm{Gd}$ & $4 \mathrm{f}^{7}$ & $7 \cdot 1 / 2$ & 0 & $7 / 2$ \\
$\mathrm{~Tb}$ & $4 \mathrm{f}^{9}$ & $5 \cdot 1 / 2$ & 5 & $15 / 2$ \\
$\mathrm{Dy}$ & $4 \mathrm{f}^{10}$ & $4 \cdot 1 / 2$ & 6 & 8
\end{tabular}

Table 2.2: $\quad$ Overview over the most common magnetic elements and their magnetic properties. For more details, see text.

In an isotropic environment the energy levels labeled by the quantum number $m_{l}$ are degenerate. As soon as the symmetry is broken though, for instance, by an external magnetic or electric field some of the $m_{l}$ degeneracy is lifted 8 The lifting of degeneracies is called Zeeman effect (for magnetic fields) respectively Stark effect (for electric fields). If the orbital is embedded in a crystal, the spatial symmetry is also broken by the crystal structure. The crystal structure induced lifting of the degeneracy is called crystal-field $(\mathrm{CF})$ splitting.

\footnotetext{
${ }^{8}$ An accidental degeneracy might still occur.
} 


\subsubsection{Crystal-field splitting}

In Ce, the $4 \mathrm{f}$ shell is partially filled and has, according to Hund's rules, a total angular momentum of $J=|L-S|=5 / 2$, see Table 2.2. In the case of $\mathrm{CeCu}_{6}$, a famous heavy-fermion system (see section 4.1), the energy level is split into three Kramers doublets because of the orthorhombic crystal structure. The energy splitting between the doublets is typically of the order of a few meV. However, the spectral width of the $4 \mathrm{f}$ band itself is, despite its strong spatial confinement, in the order of $100 \mathrm{meV}$. Hence, observing the CF splitting directly at the core level is not possible, see Fig. 2.8

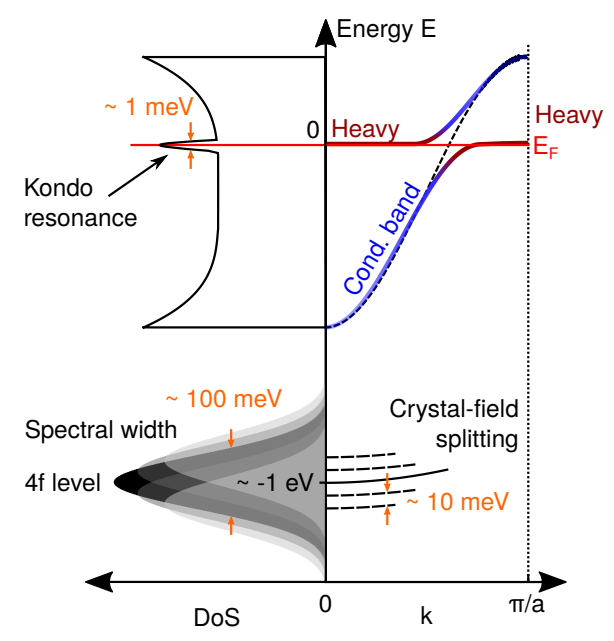

Figure 2.8.: Effects of the CF splitting on the DoS (left) and the band structure (right). Left: The DoS shows a peak at the Fermi surface due to the Kondo effect. The width of the Kondo resonance is given by $T_{\mathrm{K}}^{*}$, which is typically in the order of $1 \mathrm{meV}$ [67]. The $4 \mathrm{f}$ level, which is approximately $1 \mathrm{eV}$ below the Fermi energy, has a spectral width in the order of $100 \mathrm{meV}$. Right: At the Fermi surface, the Kondo effect causes a hybridization between the Kondo band and conduction band. The crystal symmetry leads to a partial lifting of the degeneracy of the $4 \mathrm{f}$ core level, well below the Fermi energy. The splitting is in the order of $10 \mathrm{meV}$. Because the splitting is much less than the spectral width of the $4 \mathrm{f}$ level, it cannot be observed at the orbital energy itself. However, due to the Kondo effect, the splitting is also transferred towards the Fermi surface, where it can be investigated, see Fig. 2.9.

However, as a result of the Kondo effect, some spectral weight of the $4 \mathrm{f}$ core level is transferred to the Fermi surface, leading to a sharp Lorentz-shaped resonance, see section 2.4. The spectral width of this resonance at the Fermi surface is narrow (given by the effective Kondo temperature $T_{\mathrm{K}}^{*}$ ), and is typically in the order of $1 \mathrm{meV}$ [67]. If the electron levels of the localized $4 \mathrm{f}$ orbital is split, this ultimately leads to satellite peaks surrounding the actual Kondo resonance, see Fig. 2.9. 
For $\mathrm{CeCu}_{6}$, the $\mathrm{CF}$ splitting was measured using inelastic neutron scattering. It is reported to be $\Delta_{1}=7 \mathrm{meV}$ and $\Delta_{2}=13.8 \mathrm{meV}$ at $10 \mathrm{~K} \mathrm{[68].} \mathrm{The} \mathrm{CF} \mathrm{satellites} \mathrm{are}$ symmetrically arranged around the Kondo resonance, with an energy gap given by the CF-splitting energy.
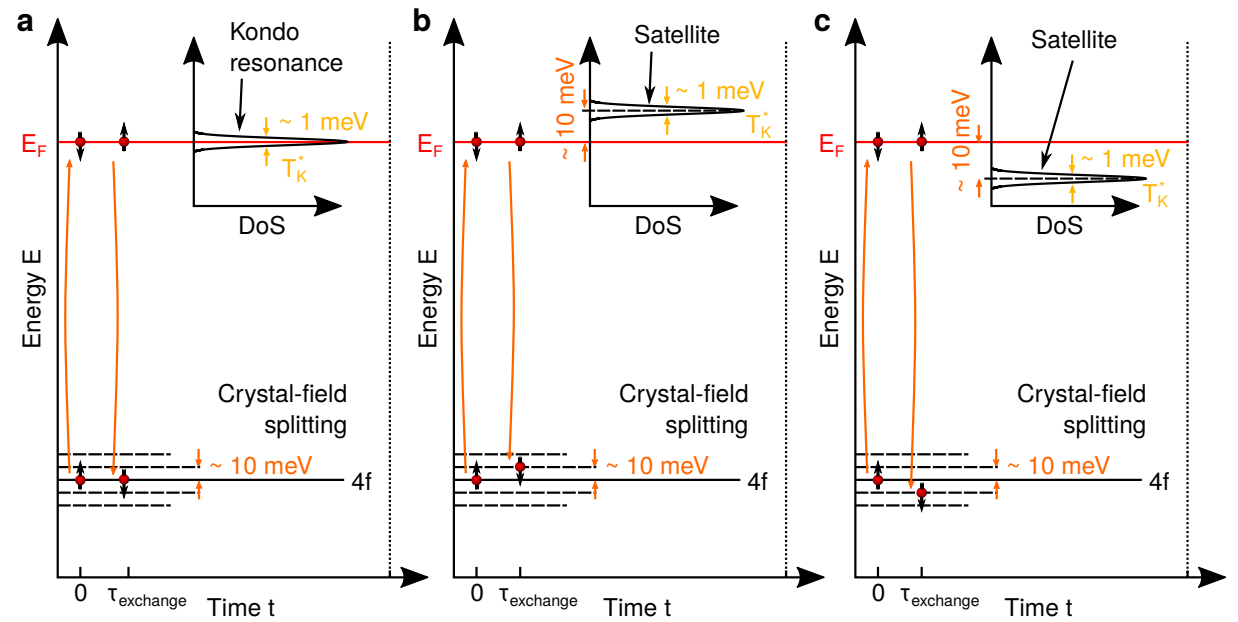

Figure 2.9.: Illustration of the exchange interaction between the localized $4 \mathrm{f}$ electrons and the conduction electrons: (a) The Kondo resonance appears due to the exchange interaction between electrons at the Fermi surface and $4 \mathrm{f}$ electrons (indicated by the orange arrows). As a direct consequence of this resonance scattering is a resonance peak at the Fermi surface. (b) Due to the CF splitting of the core level, the initial and final occupation of the $4 \mathrm{f}$ level can be in a different subband, which leads to an additional, energetically shifted resonance close to the Fermi surface. Because the resonance width is still given by $T_{\mathrm{K}}^{*}$, this resonance is clearly observed as a distinct satellite resonance, which is shifted towards higher energies, see inset. (c) Analogously, the final occupation of the uf level can be in an energetically lower state. Consequently, satellite resonances are also expected below the Fermi surface. 


\subsubsection{High-temperature Kondo effect}

The additional energy levels, due to the CF splitting, have a profound effect on the resonance at intermediate temperatures. With increasing temperatures, the Kondo spectral weight is reduced but simultaneously the energy levels are thermally broadened. At intermediate temperatures, the Kondo spectral weight is still present, but the individual CF levels will merge into one spectrally broad resonance. This new resonance is much broader and therefore has a larger effective Kondo temperature $T_{\mathrm{K}}^{*}$. This high-temperature Kondo state has been experimentally verified by transport measurements 69 75]. This change in the effective Kondo temperature $T_{\mathrm{K}}^{*}$ for intermediate temperatures provokes the definition of an effective ,high-temperature ${ }^{6}$ Kondo temperature $T_{\mathrm{K}}^{*,>}$.

Figure 2.10: The Kondo resonance at the

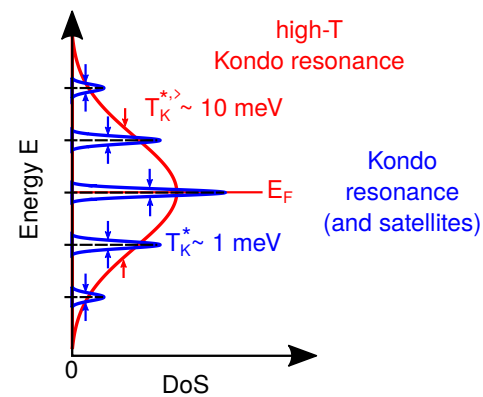
Fermi surface is surrounded by CF satellite resonances (blue). The width of the resonances is given by the Kondo temperature $T_{\mathrm{K}}^{*}$ and the spacing by the $\mathrm{CF}$ splitting, as discussed in section 2.7.1. At intermediate temperatures, these resonances broaden thermally and will turn into one, energetically much wider, resonance (red). The width of the spectrally broad resonance defines the high-temperature Kondo temperature $T_{\mathrm{K}}^{*,>}$, which is in the order of $100 \mathrm{~K}$.

Since the dynamics of the strongly correlated system is solely determined by the inverse, effective Kondo temperature, see section 2.6. two completely different time scales are expected for $T_{\mathrm{K}}^{*}$ and $T_{\mathrm{K}}^{*,>}$. Indeed, these different time scales have been experimentally verified by the author's experiment, see section 6.7 


\section{Quantum phase transitions - theoretical background}

Following the Ehrenfest classification, phase transitions are grouped into firstand second-order phase transitions [76]. First-order phase transitions show a discontinuity in the entropy, which correspondingly involve latent heat. For second-order phase transitions, the discontinuity is only observed for secondand higher-order derivatives. Because second-order partial derivatives of thermodynamic potentials correspond to response functions, the order of the phase transition can be determined by measuring thermodynamic quantities such as specific heat or magnetic susceptibility. Furthermore, second-order phase transitions are closely linked to spontaneous symmetry breaking, which come along with a continuous growth of an order parameter within the low-symmetry phase.

Most chemical phase transitions are first-order phase transitions as latent heat is needed to conduct the phase transition. In condensed matter physics on the other hand, many phase transitions obey a continuous growth of an order parameter, the distinct signature of second-order phase transitions. Well-known examples for second-order phase transitions are magnetic phase transitions as well as the transitions into the superconducting phases. This chapter is dedicated to second-order phase transitions which occur at $0 \mathrm{~K}$. If the transition temperature is suppressed to $0 \mathrm{~K}$, the phase transition is no longer driven by thermal fluctuations, but purely by quantum fluctuations. Hence, these transitions are called quantum phase transitions (QPTs). QPTs are particularly interesting as they manifest a kind of singularity in the phase diagram.

\subsection{Thermally-driven second-order phase transitions and universality classes}

Second-order phase transitions obey a universal scaling behavior. The critical order-parameter fluctuations diverge in time and space as the transition temperature is reached. Close to the phase transition temperature, the physical quantities follow a power-law behavior. To express these power laws the reduced temperature $\tau$ is defined as follows:

$$
\tau:=1-\frac{T}{T_{\text {crit }}}
$$


The specific heat $\mathrm{C}_{\mathrm{V}}$ and the magnetic susceptibility $\chi_{\mathrm{m}}$, for instance, are described by the following power-law behavior:

$$
C_{\mathrm{V}}(T) \propto|\tau|^{-\alpha}, \quad \text { and } \chi_{\mathrm{m}}(T) \propto|\tau|^{-\gamma},
$$

where $\alpha$ and $\gamma$ are called critical exponents. Critical exponents originate from a specific critical behavior in the vicinity of the phase transition. They depend on the dimensionality of the system as well as the symmetry of the coupling on which the ordering is based. Hence, many systems show identical scaling behavior in terms of critical exponents, even if the underlying processes have a completely different physical origin.

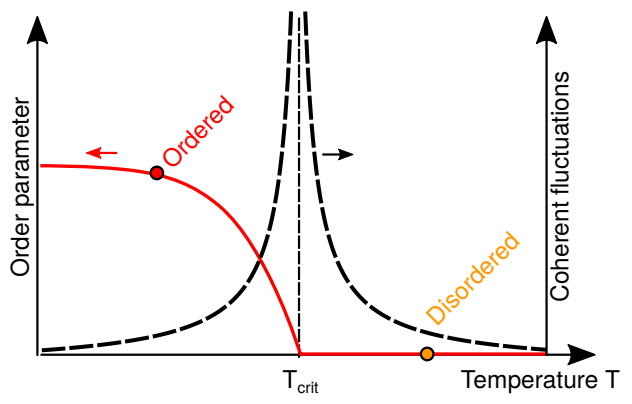

Figure 3.1: A second-order phase transition manifests in the growth of an order parameter below a temperature $T_{\text {crit }}$ (red line). As the temperature approaches $T_{\text {crit }}$, the coherent fluctuations of the order parameter are growing (dashed line). Hence, the second-order phase transitions at finite temperatures are driven by classical critical fluctuations. For more details, see text.

Critical exponents convey the universal behavior of phase transitions. At the transition temperature $\tau=0$, coherent fluctuations of the order parameter play a crucial role. These fluctuations grow and eventually diverge on the time and length scale as the phase transition temperature is reached.

As illustrated in Fig. 3.2, the region where these classical fluctuations dominate, shrinks as the transition temperature $T_{\text {crit }}$ is reduced. As $T_{\text {crit }}$ reaches $0 \mathrm{~K}$, classical thermal fluctuations are no longer driving the phase transition. This point in the phase diagram is called the quantum critical point (QCP). Even at finite temperatures, the quantum critical fluctuations (i.e. the uncertainty principle) are dominating the behavior of the system. In this quantum critical region of the phase diagram, Fermi-liquid behavior is typically no longer observed.

\subsection{Quantum phase transitions in heavy-fermions}

QPTs often occur in strongly correlated heavy-fermion systems. In the heavy-fermion compound $\mathrm{CeCu}_{6}$, von Löhneysen and co-workers discovered that by tuning an external parameter a magnetically ordered ground state establishes [10]. This tuning parameter can be chemical doping, magnetic field or pressure [10, 14]. Since the source of the heavy-fermion behavior is the Kondo effect, which is 


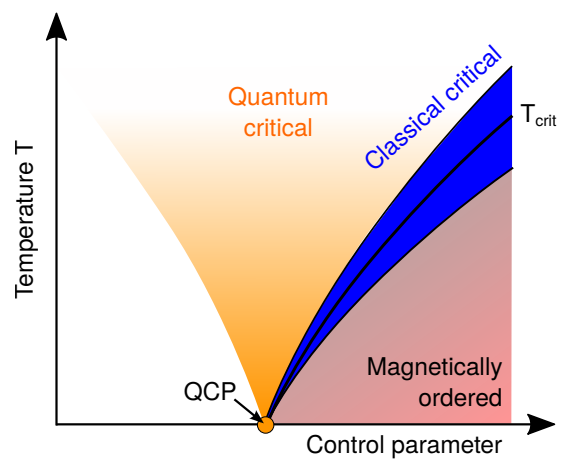

Figure 3.2: As the transition temperature $T_{\text {crit }}$ is reduced by tuning a control parameter (black line), the temperature region in which the classical critical fluctuations dominate is also reduced (blue area). As $T_{\text {crit }}$ reaches $0 \mathrm{~K}$ quantum fluctuations are dominating the system even at finite temperatures (yellow area). In this quantum critical part of the phase diagram, often non-Fermi-liquid behavior is observed. For more details, see text.

screening the magnetic moments (see chapter 2), these two distinctively different ground states are in a direct competition. At the QCP, right in between the two competing ground states, the system becomes magnetically unstable which manifests in an exceptionally high entropy. Experimentally, high entropies are directly observed in specific heat measurements, see section 4.3. A further remarkable feature of QPTs is the augury of their emergence at temperatures as high as $60 \mathrm{~K}$, far from the actual singularity (QCP) in the phase diagram [36]. This quantum critical regime provides an excellent environment for the emergence of novel ground states. Indeed, the interest in QPTs was massively boosted in by the discovery of unconventional superconductivity [2, 3, 15, 33, in magnetic systems as well as the non-Fermi-liquid behavior in close proximity of these magnetic instabilities [13].

Systems in the heavy-fermion phase show Fermi-liquid behavior, but with an unusually large effective mass $m^{*}$, see section 2.5. As the system is driven into a magnetically ordered ground state, the effective mass is greatly reduced to be again in the order of the bare electron mass, see section 2.2 The behavior at the QCP as well as above the actual QCP is controversially debated. Experimental transport measurements, for example, unravel a non-Fermi-liquid behavior by showing atypical power-law behaviors, see chapter 4 In the quantum critical regime an exquisite quantum-coherent ground state might emerge, but its details are intensely debated.

In the context of Kondo-lattice systems, the following question arises: What happens to the heavy quasiparticles in the quantum critical regime [35]? Currently, there are two types of theoretical scenarios, which are debated in the community. The task of solving these intriguing questions about QPTs is further hampered by the fact that both types of quantum criticality have been experimentally confirmed in different quantum critical systems. An overview over the two quantum critical scenarios is given in the following section 3.3 . 


\subsection{Different quantum critical scenarios}

Currently, there are two types of theoretical models for the quantum critical regime. Both scenarios predict a non-Fermi-liquid behavior, but there are some fundamental differences between the two models [12, 77]. Aggravating circumstances are the experimental evidence for both types of the suggested quantum critical scenarios. The first model is called the spin-density-wave (SDW) scenario, which was promoted by Hertz, Millis, Continentino, Moriya and Takimoto [78 81. It is also referred to as the conventional quantum critical (or Hertz-Millis) scenario.

An alternative picture of the quantum criticality was promoted by Si, Coleman, Senthil and Paul and is called the unconventional quantum criticality 35, 8284]. Based on experimental observations which are not in agreement with the Hertz-Millis scenario, they predicted an entire Fermi-surface collapse, in contrast to the partial Fermi-surface collapse caused by a SDW ground state.

\subsubsection{Conventional quantum criticality}

The conventional quantum critical scenario predicts a partial disintegration of the Fermi surface as the SDW ground state induces a hybridization gap at the Fermi surface [85. The conventional quantum criticality model forecasts scattering between the heavy quasiparticles and the emerging SDW, leading to non-Fermi-liquid behavior. Next, it should be clarified under which circumstances such SDW ground states are emerging.

A SDW ground state is generally an incommensurate alteration in the density of the spins of conduction electrons. This periodic alteration of spins is described by the so-called nesting vector $\vec{Q}$. Due to this change of periodicity, a hybridization gap $\Delta$ at the Fermi surface is formed. An illustration of Fermi surface nesting is given in Fig. 3.3. A two-dimensional free Fermi gas, for instance, has a badly nested Fermi surface, as maximally four points on the Fermi surface can be connected with a constant nesting vector $\vec{Q}$, see Fig. 3.3 (a). In the case that the Fermi surface has a more complex shape, see e.g. Fig. 3.3 (b), great portions of the Fermi surface might be connectable with a constant nesting vector $\vec{Q}$. For energetic reasons, the SDW ground state is preferential, see Eq. 3.3 and the following discussion. 

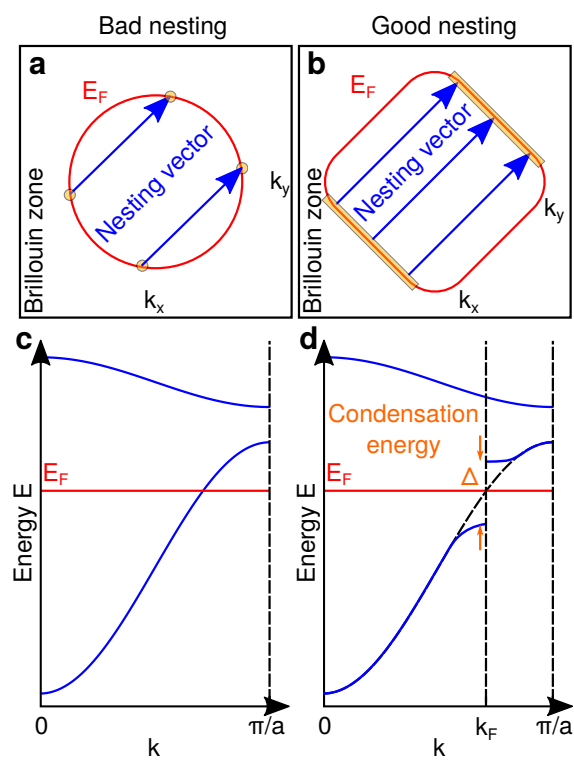

Figure 3.3: Illustration of nesting: (a) In a circularly shaped Fermi surface (two-dimensional), maximally four points (yellow) can be connected by a constant nesting vector $\vec{Q}$ (blue). (b) The shape of the Fermi surface has a great influence on the nesting properties. For this sketched Fermi surface, a great portion of the surface can be interconnected by a constant vector $\vec{Q}$. Hence, this Fermi surface is well-nested. (c) If only minor parts of the Fermi surface are nested, the formation of a hybridization gap at the Fermi surface is energetically unfavorable. (d) If the portion of the nested areas is large, the formation of a hybridization gap is energetically favorable as the condensation energy increases proportionally to $\rho\left(E_{\mathrm{F}}\right)$ of the nested areas. In this case the ground state is a SDW and the spin structure is periodically modulated with a periodicity $\vec{Q}$.

With the formation of a hybridization gap at $E_{\mathrm{F}}$ the overall energy of system can be reduced by the so-called condensation energy, which is proportional to the DoS of the nested Fermi-surface parts. Hence, larger nested areas will ultimately favor a SDW ground state:

$$
\mathcal{E}_{\text {condensation }} \propto \rho\left(E_{\mathrm{F}}\right) \Delta^{2}
$$

This explains why complex Fermi surfaces are more likely to have a SDW ground state ${ }^{1}$ However, establishing a SDW and therefore distorting a magnetic structure costs energy. Hence, a system only shows a SDW ground state if the gained condensation energy exceeds the energy expenses required for the distortion.

Due to the high DoS at $E_{\mathrm{F}}$ in Kondo-lattice systems (see section 2.5.1), they are predestined to show SDW ground states. Since only parts of the Fermi surface are vanishing due to the formation of a hybridization gap, not all quasiparticles are necessarily affected by the partial Fermi-surface destruction. However, the strongly enhanced SDW-quasiparticle scattering cross section is sufficient to

\footnotetext{
${ }^{1}$ Furthermore, also the Peierls instability can be understood in terms of nesting, since a one-dimensional metallic chain consists of two parallel planes as its Fermi surface. Hence, this perfect nesting causes a hybridization gap at the entire Fermi surface which interferes the system's metallic property (Fermi-surface collapse) [86].
} 
induce non-Fermi-liquid behavior. Examples of heavy-fermion systems where predictions from the SDW scenario are in excellent agreement with experimental observations are, for instance, $\mathrm{CeCu}_{2} \mathrm{Si}_{2}, \mathrm{CeRu}_{2} \mathrm{Si}_{2}$ and $\mathrm{Ce}_{1-x} \mathrm{La}_{x} \mathrm{Ru}_{2} \mathrm{Si}_{2}$ [12]. The explanation of how a SDW ground state manifests in the experiments is explained in chapter 4 .

\subsubsection{Unconventional quantum criticality}

Despite the experimental evidence of the SDW scenario in some heavy-fermion compounds, there are also systems which do not comply with the predictions of the SDW scenario. Famous examples are the quantum critical systems $\mathrm{CeCu}_{5.9} \mathrm{Au}_{0.1}$ and $\mathrm{YbRh}_{2}\left(\mathrm{Si}_{0.95} \mathrm{Ge}_{0.05}\right)_{2}$ [13, 87]. The experimentally observed behavior for these systems is not in agreement with the Hertz-Millis scenario. Because of this mismatch of the experiments and the theory these systems are often called unconventional quantum critical.

Neutron scattering experiments allow to determine the magnetic structure as function of the momentum vector $\vec{k}$ [88. As explained before, a SDW ground state affects only the nested areas of the Fermi surface and therefore a strong anisotropy in the spin fluctuations (in the momentum space) is expected [12]. However, in unconventional quantum critical systems the correlated spin fluctuations are not only confined to a single nesting vector $\vec{Q}$, but they are observed along multiple directions. This is direct experimental evidence that these systems cannot have a SDW ground state. In fact, these neutron experiments suggest a full destruction of the Fermi surface as only then an isotropic response (in momentum space) is expected. The neutron scattering experiments on $\mathrm{CeCu}_{5.9} \mathrm{Au}_{0.1}$ further revealed that the correlated spin fluctuations are mainly confined to the $a^{*} c^{*}$ plane in momentum space [88, 89].

The driving mechanisms for such processes are intensely debated. However, a destruction of heavy fermions might be explained by the separation of spins and charges, which would ultimately lead to a break down of the well-established particle concept. Because the entire Fermi surface collapses, the unconventional quantum criticality is also called localized breakdown scenario ${ }^{2}$ A localized destruction of the heavy-fermion state means that in the quantum critical regime the Kondo screening is fully destroyed. This model furthermore suggests that the existence of quasiparticles and the unconventional quantum critical ground state are mutually exclusive, which is the major difference with respect to the conventional quantum critical scenario.

\footnotetext{
${ }^{2}$ Note that an isotropic response in the momentum space corresponds to a delta-peak in real space. Therefore, the isotropic neutron scattering response suggests a local break down of every screened magnetic moment.
} 
Summary and Perspective. $\mathrm{YbRh}_{2} \mathrm{Si}_{2}$ and $\mathrm{CeCu}_{6}$ are two well-known and extensively discussed heavy-fermion systems which show unconventional quantum criticality under adequate conditions. The time-resolved terahertz experiments presented in this thesis provide a new perspective which could help understanding better quantum critical behavior. Unconventional quantum critical behavior has been demonstrated by many different techniques, such as transport measurements, inelastic neutron scattering and scanning tunneling microscopy (see chapter (4). Typically, a collapse of the Kondo spectral weight at sufficiently low temperatures is observed, which indicates unconventional quantum criticality. In contrast to these established methods, the time-resolved terahertz ansatz allows us to monitor the picosecond dynamics of the system. Because of the low Kondo temperature in $\mathrm{CeCu}_{6}$ (see section 4.1.2), the characteristic time scale of the strong correlation dynamics is expected to be in the order of picoseconds (see section 6.4. The picosecond dynamics furthermore allows us to investigate the Kondo spectral weight, while simultaneously and directly monitoring the effective Kondo temperature of the system, see chapters 6 and 7 



\section{Heavy-fermion systems and experimental approaches}

Generally, there has been a increasing interest in strong correlation physics over the last decades. As a result many different experimental ways of measuring and accessing strongly correlated ground states have been developed. This chapter is dedicated to give an overlook over the different experimental techniques, which are suitable for investigating strongly correlated heavy-fermion systems as well as the associated quantum critical regime. Furthermore, the two systems $\mathrm{CeCu}_{6-x} \mathrm{Au}_{x}$ and $\mathrm{YbRh}_{2} \mathrm{Si}_{2}$ will be explained as they were used to conduct the experiments presented in this thesis, see chapters 6 and 7 . In the thesis at hand an additional method is introduced which allows for a different and complementary perspective onto quantum criticality.

\section{1. $\mathrm{CeCu}_{6-x} \mathrm{Au}_{x}$}

The first $\mathrm{CeCu}_{6}$ single crystals were grown by Stewart and co-workers in 1984 [5]. They observed a temperature-dependent magnetic susceptibility and specific heat, which is only in good agreement with Fermi-liquid theory if a massively increased effective electron mass $m^{*}$ is assumed. This, together with the same qualitative observations in $\mathrm{CeAl}_{3}$ [1 and in $\mathrm{UBe}_{13}$ [3], led to the term ,heavy-fermion'. Germann and co-workers noticed in 1988 that by chemically doping $\mathrm{CeCu}_{6}$ with $\mathrm{Au}$, the system can be driven into an antiferromagnetic ground state at sufficiently low temperatures. Hence, they created the $\mathrm{CeCu}_{6-\mathrm{x}} \mathrm{Au}_{\mathrm{x}}$ heavy-fermion family [20]. Since the Kondo-lattice effect is driving the heavy-fermion behavior, magnetic ordering cannot emerge simultaneously. This means that for a critical doping the appearance of a QPT and the associated non-Fermi-liquid behavior is expected, see chapter 3 . The critical doping threshold was identified to be $x \approx 0.1$ [13, 89]. $\mathrm{CeCu}_{5.9} \mathrm{Au}_{0.1}$ is known to be an unconventional quantum critical system [88.

\subsubsection{The basic alloy: $\mathrm{CeCu}_{6}$}

$\mathrm{CeCu}_{6}$ shows no long-range magnetic ordering down to $20 \mathrm{mK}$ 90. It was only in the year 2000 when a magnetic phase transition at $2 \mathrm{mK}$ was proven with AC magnetic susceptibility and thermal expansion measurements [91. However, the heavy-fermion behavior sets in well above the $\mathrm{mK}$ temperature range and is assumed to be unaffected by this magnetic transition 36, 92. 
$\mathrm{CeCu}_{6}$ has an orthorhombic Pnma structure (space group number 62) at room temperature. As the crystal is cooled, the system undergoes a weak orthorhombic-to-monoclinic distortion, which takes place at approximately $220 \mathrm{~K}$, leading to a magnetic anisotropy [93. The low-temperature space group is $\mathrm{P} 21 / \mathrm{c}$ (space group number 14). This crystallographic distortion plays only a minor role in the formation process of heavy fermions and the subsequent quantum critical behavior. The spacing between the magnetic Ce atoms is the most relevant parameter for deciding whether the system shows a heavy fermion or a magnetically ordered ground state. However, the crystal symmetry is relevant for the CF splitting, as the crystal symmetry causes a partial lifting of the degeneracy of the core levels, see section 2.7. In $\mathrm{CeCu}_{6}$ the energy level of the partially filled $4 \mathrm{f}$ core of the Ce atoms is lifted into three Kramers doublets.

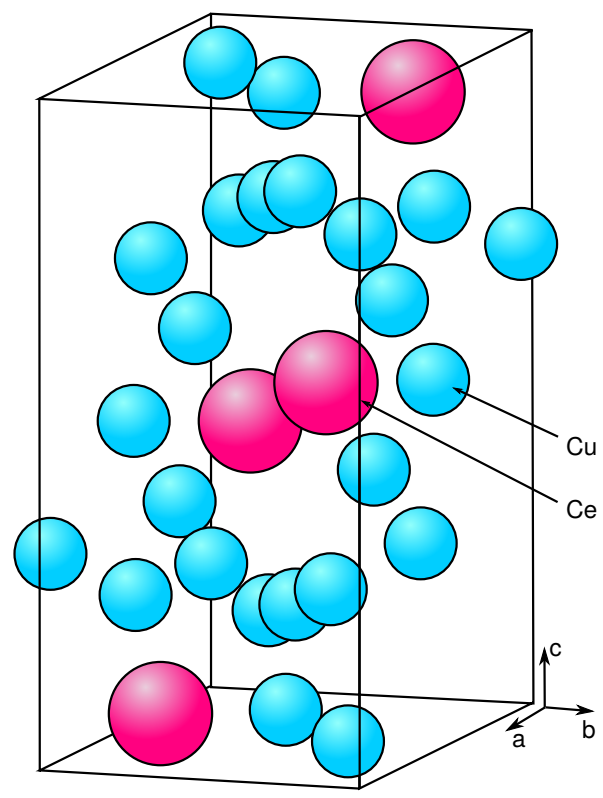

Figure 4.1: Crystal structure of $\mathrm{CeCu}_{6}$ at room temperature is the orthorhombic Pnma phase (space group number 62) 94. For temperatures below approximately $220 \mathrm{~K}$, a slight distortion takes place and the orthorhombic crystal structure turns into a monoclinic P21/c structure (space group number $14)$.

\subsubsection{Au doping: $\mathrm{CeCu}_{6-x} \mathrm{Au}_{x}$}

Doping the $\mathrm{CeCu}_{6}$ single crystal with slightly larger $\mathrm{Au}$ atoms on the $\mathrm{Cu}$ sites has two effects: The Pnma crystal structure of $\mathrm{CeCu}_{6}$ is stabilized for lower temperatures and the fine balance between RKKY coupling and the Kondo effect is tuned [95]. In fact, by exchanging some $\mathrm{Cu}$ atoms with larger $\mathrm{Au}$ atoms an antiferromagnetic ground state establishes at sufficiently low temperatures [13. The distortion caused by the chemical doping induces a reduction of the hybridization between the $4 \mathrm{f}$ orbitals and the conduction electrons, triggered by a decreasing spin-exchange constant $J$. This weakened spin exchange leads to an improper Kondo screening of localized magnetic moments, which suddenly 
enables the emergence of an antiferromagnetic ordering via the RKKY interaction, section 2.4.1.

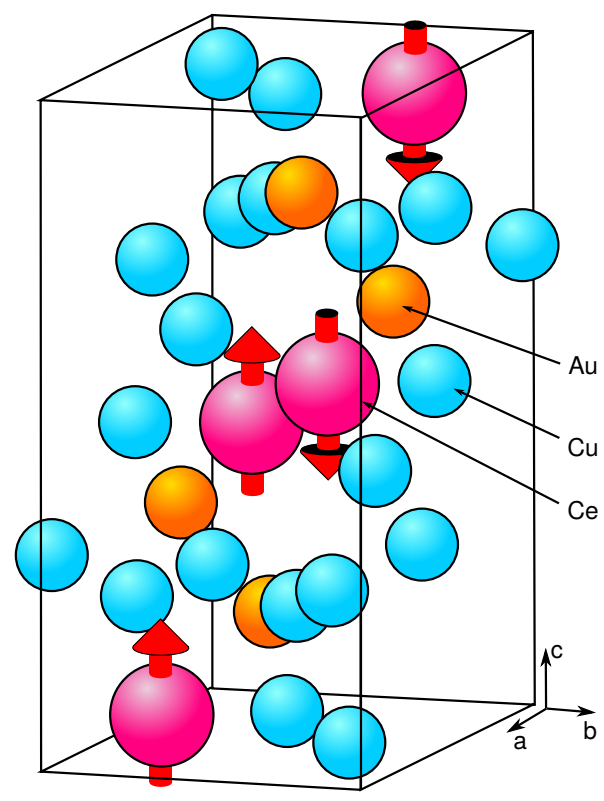

Figure 4.2: The crystal structure of $\mathrm{CeCu}_{5.0} \mathrm{Au}_{1.0}$ is the orthorhombic Pnma phase (space group number 62) 96. At sufficiently low temperatures, an incommensurate antiferromagnetic phase emerges, indicated by the red arrows. Note that the magnetization on each lattice site is different, indicated by the arrow lengths.

Figure 4.3: Doping-dependent Néel

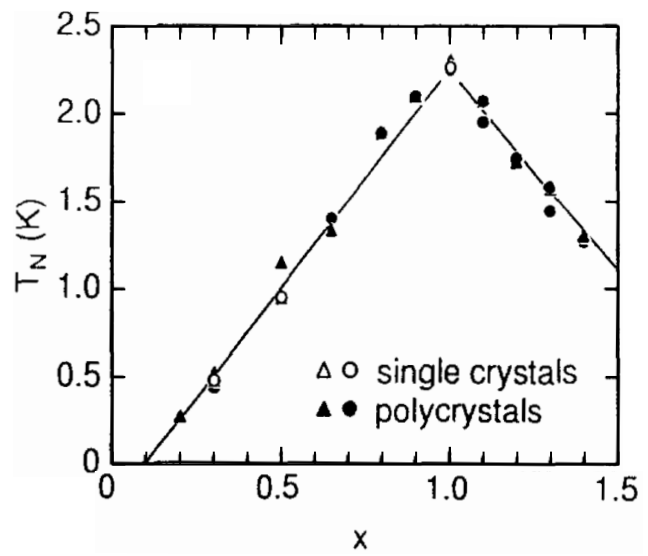

temperature for $\mathrm{CeCu}_{6-x} \mathrm{Au}_{x}$ compounds. For dopings larger than $x=0.1$, an incommensurate antiferromagnetic phase establishes. At low doping concentrations a heavy-fermion state deploys. At the interface between the two regions $(x=0.1)$, a peculiar quantum critical regime is formed. The system in this range does no longer behave like a Fermi liquid, see section 3.3.2 Reprinted with permission from [95].

For $\mathrm{Au}$ dopings larger than $x>0.1$ an incommensurate magnetic ordering is observed. This magnetic ordering leads to a sharp maximum in the specific heat $C_{\mathrm{V}}$ at the phase transition [20]. The suspected incommensurate ordering was later confirmed by neutron scattering experiments [97. For Au dopings $x<1.0$ only one specific $\mathrm{Cu}$ site is partially occupied by the Au atom, see Fig. 4.2 . In this range of chemical doping, the Néel temperature $T_{\mathrm{N}}$ linearly changes with doping: 


$$
T_{\mathrm{N}} \approx 2.2 \mathrm{~K} \cdot x-0.22 \mathrm{~K}, \quad x \in[0.1,1.0]
$$

For Au concentrations $x>1.0$, the Néel temperature again decreases due to subtle changes in the orthorhombic crystal structure, as a second $\mathrm{Cu}$ site starts to be substituted by the $\mathrm{Au}$ atoms 95. The following table illustrates the qualitative change of the lattice parameters as the level of chemical doping is increased $(\uparrow$ : increase and $\downarrow$ : decrease):

\begin{tabular}{c||c|c|c}
$\mathrm{x}$ & $\mathrm{a}$ & $\mathrm{b}$ & $\mathrm{c}$ \\
\hline $0.1 \rightarrow 1.0$ & $\uparrow$ & $\uparrow$ & $\downarrow$ \\
$1.0 \rightarrow 2.0$ & $\uparrow$ & $\uparrow$ & $\uparrow$
\end{tabular}

Note that the orthorhombic-to-monoclinic structural phase transition only appears for dopings $x<0.1498$. On the other hand, by applying hydrostatic pressure, the effective doping concentration can be reduced. Hence, applying hydrostatic pressure to $\mathrm{CeCu}_{6-\mathrm{x}} \mathrm{Au}_{x}$ single crystals is equivalent to reducing the effective chemical doping.

The Kondo temperature $T_{\mathrm{K}}^{*}$ for $\mathrm{CeCu}_{6}$ is reported to be between $6 \mathrm{~K}$ and $7 \mathrm{~K}$ [7, 14, 36, 99-102]. Note that the value of this effective Kondo temperature slightly changes, depending on the experimental method used to determine it. The Kondo temperature for any heavy-fermion system is expected to be in the lower Kelvin range $\left(T_{\mathrm{K}}^{*} \sim 10 \mathrm{~K}\right)$. The reason is to be found in the characteristic short-range spatial correlation of the Kondo disorder, which was experimentally observed in muon spin rotation experiments on $\mathrm{CeCu}_{5.9} \mathrm{Au}_{0.1}$ as well as on $\mathrm{UCu}_{5-x} \mathrm{Pd}_{x}$ samples [67.

\section{2. $\mathrm{YbRh}_{2} \mathrm{Si}_{2}$}

The heavy-fermion nature of $\mathrm{YbRh}_{2} \mathrm{Si}_{2}$ was only discovered in 2000. It is the first Yb-based compound showing non-Fermi-liquid behavior [54]. $\mathrm{YbRh}_{2} \mathrm{Si}_{2}$ orders magnetically below $70 \mathrm{mK}$, as it was demonstrated by magnetic susceptibility measurements as well as neutron scattering experiments [54, 103]. Features indicating phase transitions are also observed in the specific heat as well as in the electrical resistivity [34. Above $200 \mathrm{~K}$ the system follows the Curie-Weiss behavior: $\chi_{\mathrm{m}} \propto \Theta /\left(T+T_{\mathrm{N}}\right)$. At $70 \mathrm{mK}$ the Curie-Weiss temperature $\Theta$ is anisotropic ${ }^{1}$ which indicates an easy-plane magnetic ordering. In contrast, Ce-based heavy-fermion compounds are easy-axis systems. $\mathrm{YbRh}_{2} \mathrm{Si}_{2}$ crystallizes in a tetragonal I 4/mmm crystal structure (space group number 139 ) and does not show any structural phase transitions.

${ }^{1}$ The Curie-Weiss temperature $\Theta_{\|}$along c-axis is orders of magnitude larger than its in-plane value $\Theta_{\perp}$. 

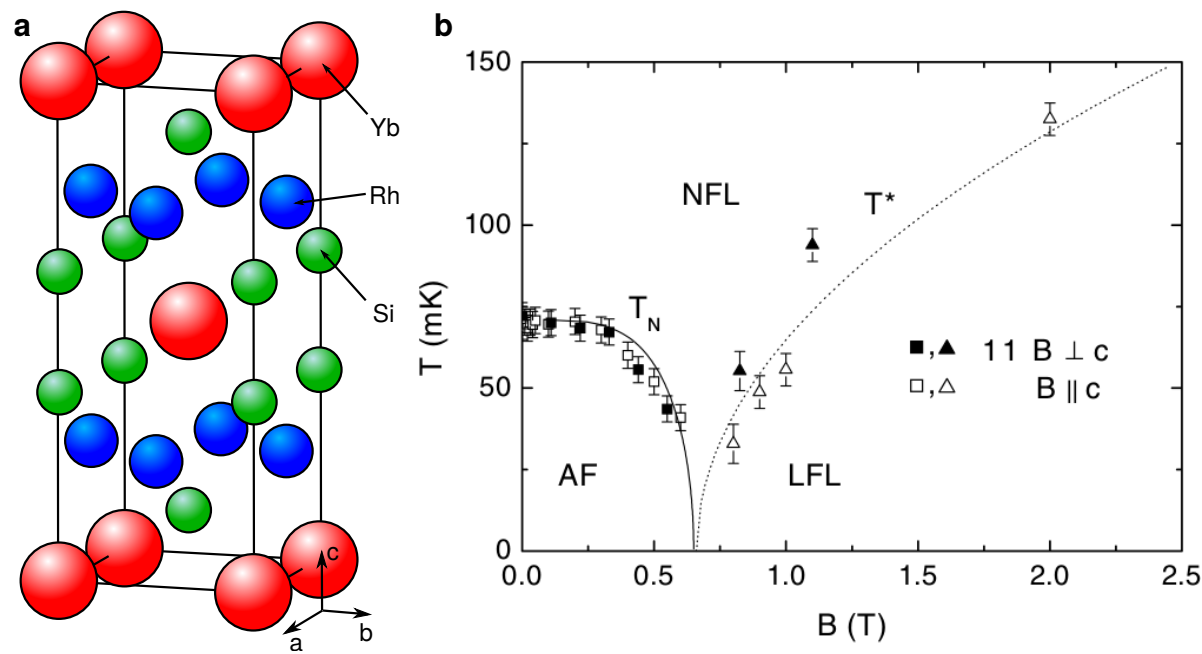

Figure 4.4.: (a) Tetragonal unit cell of $\mathrm{YbRh}_{2} \mathrm{Si}_{2}$ [104. (b) Corresponding phase diagram at function of an external control parameter, which can be either Ge-doping or magnetic field. Fig. (b) reprinted with permission from [34].

Undoped $\mathrm{YbRh}_{2} \mathrm{Si}_{2}$ does not show quantum critical behavior. However, by applying a magnetic field of $0.7 \mathrm{~T}$ [105] or by Ge doping, the system becomes quantum critical. In $\mathrm{YbRh}_{2}\left(\mathrm{Si}_{1-x} \mathrm{Ge}_{x}\right)_{2}$, the critical doping to suppress the magnetic ordering is reported to be $x=0.05$ [106].

\subsection{Experimental techniques}

As explained in section 2.5.1 the Kondo effect leads to a spectral weight shift of the localized $4 \mathrm{f}$ magnetic moments to the Fermi surface. The appearance of a Kondo spectral weight ultimately results in a hugely increased DoS at the Fermi energy. Already in the simplified non-interacting Fermi-gas picture of metals, it becomes obvious that the electronic transport quantities such as electrical conductivity, magnetic susceptibility or specific heat measurements are directly linked to the DoS at the Fermi surface. Hence, using transport measurements to investigate heavy-fermion systems becomes self-evident. Also $\mathrm{CeCu}_{6}$ and $\mathrm{YbRh}_{2} \mathrm{Si}_{2}$ were discovered and identified as heavy-fermion compounds using transport measurements [5, 54. In the heavy-fermion regime of the compounds, though, the electronic properties resemble a Fermi-liquid behavior, if the effective electron mass $m^{*}$ is strongly rescaled.

As the heavy-fermion systems are driven into the quantum critical regime, the Fermi-liquid behavior is no longer observed. In transport experiments non-Fermi-liquid behavior manifests in anomalies such as a different temperature 
dependences. However, it is worth mentioning that transport experiments are rather indirect methods and more direct experimental tools would be beneficial and desirable. Here, a brief overview over the most relevant and established experimental techniques is given.

\subsubsection{Transport measurements}

Specific heat measurements. The specific heat of a metal has two major contributions: the electronic degrees of freedom as well as the lattice vibrations. For a metal at sufficiently low temperatures (i.e. $T \ll T_{\mathrm{F}}$ ), only the electronic degrees of freedom contribute to the specific heat $C_{\mathrm{V}}$. Hence, $C_{\mathrm{V}}$ is expected to be linear in temperature for Fermi liquids. The proportionality factor is called the Sommerfeld constant $\gamma$, which scales linearly with the DoS at the Fermi surface [55]:

$$
C_{\mathrm{V}}=\gamma T, \quad \text { where } \gamma=\frac{\pi^{2}}{3} k_{\mathrm{B}}^{2} \rho\left(E_{\mathrm{F}}\right)
$$

In heavy-fermion systems this linearity persists, but has to be strongly renormalized. This renormalization factor gives access to the effective mass $m^{*}$ of the heavy fermion as the Sommerfeld constant is proportional to the DoS at $E_{\mathrm{F}}$ [55]. However, as a heavy fermion is driven into the quantum critical regime, the Fermi-liquid behavior is no longer observed. The experimentally observed temperature-dependent specific heat in the quantum critical regime shows a logarithmically diverging behavior ${ }^{2}$ [13, 89, 105]:

$$
C_{\mathrm{V}}(T)=T \ln \left(\frac{T_{0}}{T}\right)
$$

Hence, $C_{\mathrm{V}}$ can be used to distinguish Fermi liquids from non-Fermi liquids as well as for determining the effective mass $m^{*}$. Measuring the specific heat $C_{\mathrm{V}}$ is particularly interesting, since it is directly coupled to the entropy $S$ of the system. By integrating the specific heat $C_{\mathrm{V}}$ over the temperature $T$, the entropy $S$ can be directly determined [55]:

$$
S(T)=\int_{0}^{T} C_{\mathrm{V}}(\tau) \mathrm{d} \tau
$$

Hence, the unusually high specific heat $C_{\mathrm{V}}$ translates into a high entropy, which corresponds to a large disorder. Intuitively it is clear that disordered phases are prone to undergo a disordered-ordered phase transition as the system is cooled. From this point of view, the emergence of exquisite phases in close proximity of the QCP can be anticipated.

${ }^{2}$ Three-dimensional antiferromagnetic structures with two-dimensional fluctuations show a logarithmic temperature dependence in $C_{\mathrm{V}} / T$. The emergence of two-dimensional fluctuations (in three-dimensional structures) is far from fully clarified [12, 107, 108. 


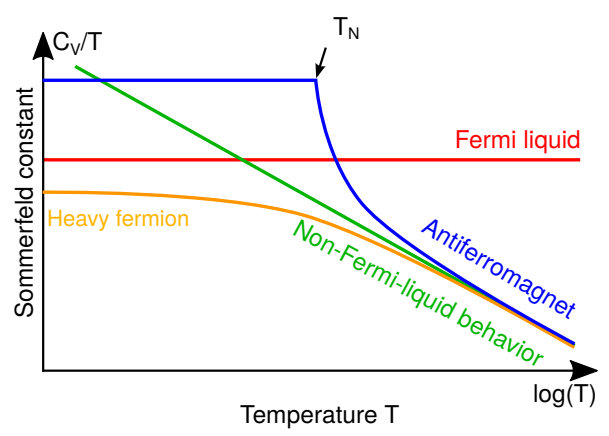

Figure 4.5: Qualitative illustration of the temperature-dependent Sommerfeld constant $\gamma=C_{\mathrm{V}} / T$. For a Fermi liquid, the Sommerfeld constant is independent of temperature (red). For quantum critical systems, a logarithmic divergence of the specific heat is expected (green), see Eq. 4.3. If the system undergoes an antiferromagnetic phase transition, $C_{\mathrm{V}} / T$ shows a sharp kink at the Néel temperature $T_{\mathrm{N}}$ (blue). On the other hand, if the system shows a heavy-fermion behavior, the well-known Fermi-liquid behavior establishes as the temperature approaches $0 \mathrm{~K}$ (yellow) [12, 14].

Magnetization measurements. Since the Kondo effect is based on the spin-exchange interaction between the localized magnetic moments and the free conduction electrons, the magnetic-field dependence is also interesting. The linear magnetic susceptibility $\chi$ is defined as the field normalized magnetization change. In a Fermi liquid the magnetic susceptibility is proportional to the DoS at the Fermi surface:

$$
\chi_{\mathrm{m}}(T):=\frac{\Delta M(T)}{H}=\mu_{\mathrm{B}}^{2} \rho\left(E_{\mathrm{F}}\right) .
$$

For the quantum critical $\mathrm{CeCu}_{5.9} \mathrm{Au}_{0.1}$ though, the experimentally observed temperature-dependent magnetic susceptibility has the following functional behavior [13]:

$$
\chi_{\mathrm{m}}(T)=\chi_{0}-\alpha \sqrt{T},
$$

where $\alpha$ is an experimentally determined system parameter. The experimentally determined parameter $\alpha$ for $\mathrm{CeCu}_{5.9} \mathrm{Au}_{0.1}$ is 0.26 [13]. As a direct consequence, the magnetic susceptibility can also be used to verify the Fermi-liquid behavior. Furthermore, the magnetic susceptibility shows anomalies at any magnetic phase transition which are useful features to detect the antiferromagnetic phase boundaries as well as heavy-fermion behavior, see Fig. 4.6. 


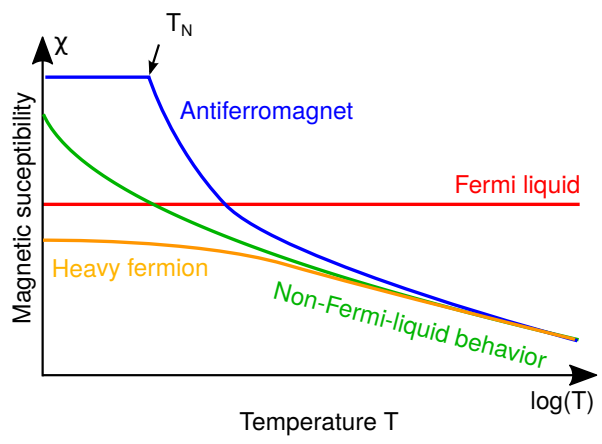

Figure 4.6: Fermi liquids show a temperature-independent magnetic susceptibility (red). In the case of a non-Fermi-liquid behavior in quantum critical systems (green), the magnetic susceptibility rises with decreasing temperature [13. If the system is tuned to undergo a magnetic phase transition, the magnetic susceptibility shows a sharp kink at the Néel temperature $T_{\mathrm{N}}$ (blue). If the system is a heavy fermion, the well-known Fermi-liquid behavior establishes as the temperature approaches $0 \mathrm{~K}[14,101]$.

Electrical resistivity measurements. As the Kondo screening sets in, the number of free electrons slightly decreases. Additionally and even ore importantly, the scattering cross section between the quenched localized magnetic moments (Kondo singlets) and the conduction electrons is greatly enhanced, causing an increase in the electrical resistivity with decreasing temperature. This scattering increase as the sample is cooled causes a minimum in the temperature-dependent resistivity, the key signature of the Kondo effect. For Fermi liquids, the temperature-dependent resistivity is expected to scale quadratically with temperature [109]:

$$
R(T)=R_{0}+A T^{2},
$$

where $R_{0}$ is the remnant resistivity at $0 \mathrm{~K}$ and $A$ scales with the Sommerfeld constant squared [13]. The remnant resistivity results from the quasiparticle-quasiparticle scattering of the Fermi liquid. In contrast to the heavy Fermi-liquid behavior of $\mathrm{CeCu}_{6}$, the quantum critical system $\mathrm{CeCu}_{5.9} \mathrm{Au}_{0.1}$ shows a linear temperature dependence in the electrical resistivity, an unambiguous signature of non-Fermi-liquid behavior. 


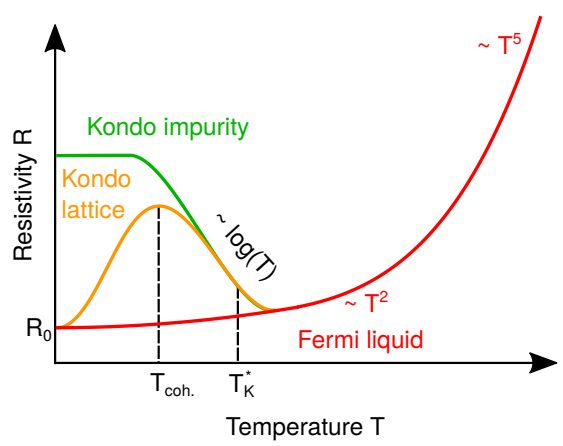

Figure 4.7: The resistivity $R$ of an ordinary metal scales with $T^{2}$ at sufficiently low temperatures (red), at which the electron-electron scattering dominates $\left(T<\Theta_{\text {Debye }}\right)$. If the system contains dilute magnetic impurities, at sufficiently low temperatures the Kondo effect manifests in a resistivity minimum (green). As the temperature approaches $0 \mathrm{~K}$, the resistivity becomes constant. If the magnetic impurities are on a dense lattice (yellow), at temperatures below $T_{\text {coh. }}$ the lattice coherence sets in, causing again a reduction in the electrical resistivity [11, 110, 111].

\subsubsection{Elastic neutron scattering}

An established method for investigating magnetic structures is elastic neutron scattering. The spin fluctuations can be analyzed as function of the wave vector $\vec{q}$, the energy transfer $E$ and the sample temperature $T$. Neutron scattering experiments allow to extract the wave vector and energy-dependent magnetic response $S$ [12, 88].

If the magnetic response $S^{\alpha}$ of the quantum critical $\mathrm{CeCu}_{5.9} \mathrm{Au}_{0.1}$ compound is plotted as function of $E / T$, the data collapse onto a single curve, see Fig. 4.8. Note that the exponent $\alpha$ for $\mathrm{CeCu}_{5.9} \mathrm{Au}_{0.1}$ is 0.75 , which manifests once again the peculiar behavior of this compound. This so-called $E / T$ scaling behavior is only expected for unconventional quantum critical systems, but not for the Hertz-Millis scenario 88 .

Elastic neutron scattering is a powerful method to distinguish the fundamentally different quantum critical scenarios as it gives direct access to the momentum-resolved spin fluctuations. Furthermore, a wave vector independent magnetic response $S$ hardens the suspected localized breakdown scenario (see section 3.3.2. A wave vector independence is equivalent to strongly confined local effect in real space, which means that all Kondo singlets are locally destroyed, as the real and the momentum space are connected via the Fourier transform [12, 88]. 


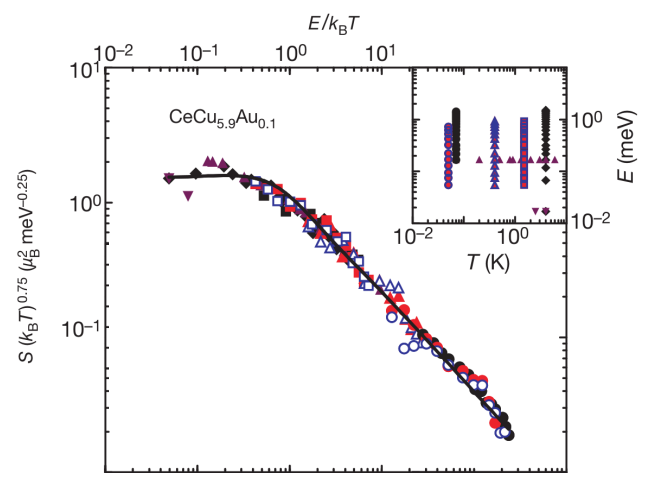

Figure 4.8: Measurements of the spin fluctuations $S$ for quantum critical $\mathrm{CeCu}_{5.9} \mathrm{Au}_{0.1}$. Due to the data collapse by plotting $S^{\alpha}$ vs. the temperature normalized energy transfer $E / T$, a SDW ground state can be excluded. The temperature $T$ as well as the transfer energy $E$ have been tuned over more than one order of magnitude (see inset), and the $E / T$ scaling is still valid. Note that the scaling collapse is only realized for $\alpha=0.75$, a direct consequence of the unusual behavior of the quantum critical $\mathrm{CeCu}_{5.9} \mathrm{Au}_{0.1}$. Reprinted with permission from 88 .

\subsubsection{Scanning tunneling microscopy}

Scanning tunneling spectroscopy (STS) is a spectroscopic imaging technique with atomic spatial resolution and it is also used to investigate Kondo physics. STS is further able to energetically resolve the local DoS of the electronic states in the vicinity of the Fermi surface. A bias voltage between the sample and the tip determine the relative probing energy with respect to the Fermi surface $E_{\mathrm{F}}$. For performing STS, an additional oscillating voltage is applied between the STM tip and the sample. Using a lock-in amplifier, the so-called differential conductance $\mathrm{d} I / \mathrm{d} V$ can be extracted as a function of the constant bias voltage and the tip position. The differential conductance is proportional to the DoS at the energy defined by the bias voltage $V_{\text {bias }}$ [112]

$$
\frac{\mathrm{d} I}{\mathrm{~d} V} \propto-\rho\left(E_{\mathrm{F}}+|e| V_{\mathrm{bias}}\right)
$$

Thanks to an atomically sharp spatial resolution and the ability to apply bias voltages in the sub-mV range, STS allows for a detailed analysis of the DoS in the energy range relevant for Kondo physics.

STS was used to investigate Kondo impurities as well as Kondo lattices. Furthermore, also the most basic physical system obeying quantum criticality was realized using a scanning tunneling microscope (STM): A magnetic atom on a metallic surface can be scanned using a magnetic tip. Three milestone STM experiments in the context of Kondo physics are subsequently presented. 


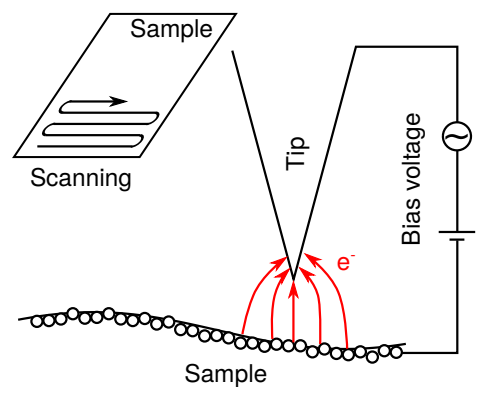

Figure 4.9: In scanning tunneling microscopy an atomically sharp tip is used to measure the tunneling current (indicated by red arrows). To perform spectroscopy, an oscillating voltage with a DC offset is applied between tip and sample. By measuring the differential conductance $\mathrm{d} I / \mathrm{d} V$ as function of the bias voltage $V, \rho\left(E_{\mathrm{F}}+|e| V\right)$ is directly accessed. Hence, STS is a spatially resolved spectroscopy tool with sub-meV energy resolution.

Observation of Kondo-impurity effect. The first STS experiment on a Kondo-impurity system was performed by Madhavan and co-workers in 1998 [11]. They used a low-temperature STM to investigate magnetic Co atoms placed on an $\mathrm{Au}(111)$ surface, the prototypical Kondo-impurity system. They spatially imaged the Co atoms and performed STS studies at various lateral tip positions relative to a Co impurity. At the position of a Co atom a clear resonance at the Fermi surface is observed which corresponds to the Kondo spectral weight allocated in close proximity of the Fermi surface. The resonance shape is asymmetric with respect to the bias voltage, see Fig. 4.10. The asymmetry originates from the fact that the tunneling current is not only limited to the Co atom. Parts of the tunneling current are due to the surrounding conduction band which causes additional quantum-interference effects leading to a so-called asymmetric Fano resonance [113, 114.

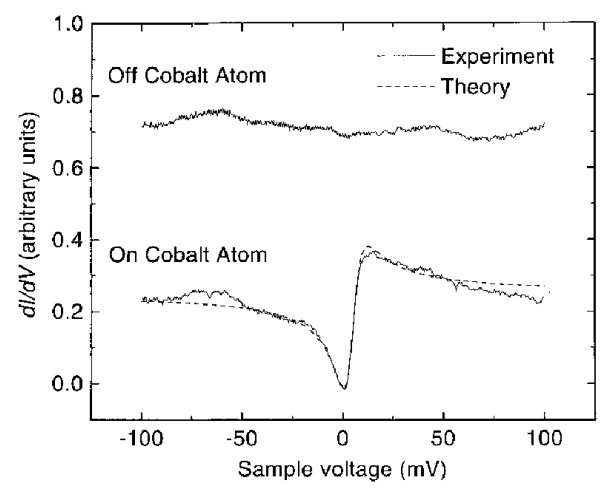

Figure 4.10: Differential conductance signals $\mathrm{d} I / \mathrm{d} V$ of an Au tip measuring a on $\mathrm{Au}(111)$ surface. If the tip is located above a magnetic Co atom, which is located on the $\mathrm{Au}(111)$ surface, a Fano resonance emerges. If the tip is far from any Co atom, no resonance appears. Reprinted with permission from [113.

A positive bias voltage $V_{\text {bias }}>0$ refers to a tunneling of electrons from the sample into the tip and therefore monitoring the electronic structure below the Fermi surface. By applying a negative bias voltage $V_{\text {bias }}<0$, the electrons are tunneling from the tip into the sample monitoring the hole-like structure. 
Observation of a Kondo lattice and crystal-field satellites. In 2011, the Kondo-lattice system $\mathrm{YbRh}_{2} \mathrm{Si}_{2}$ was investigated using STS. In contrast to Kondo-impurity systems, the Kondo resonance is expected to be surrounded by CF satellites originating from the symmetry of the lattice (see section 2.7), which was experimentally confirmed by Ernst and co-workers, see Fig. 4.11 and [115.

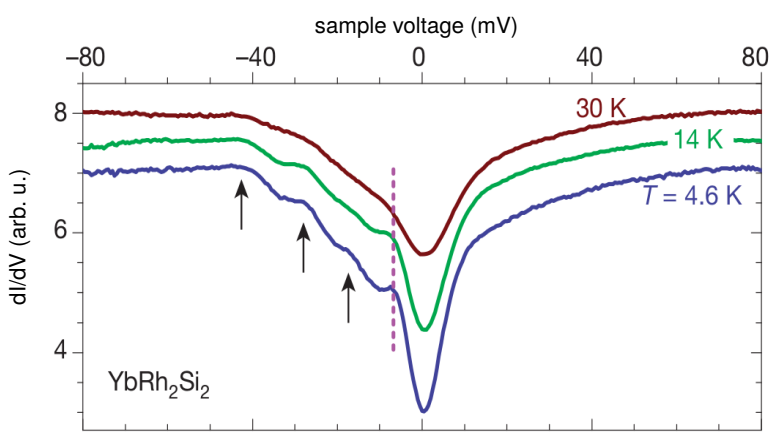

Figure 4.11.: Temperature-dependent differential conductance measurement on an $\mathrm{Yb}$ atom in $\mathrm{YbRh}_{2} \mathrm{Si}_{2}$. The clear and strong dip around $0 \mathrm{~V}$ corresponds to the Kondo resonance at $E_{\mathrm{F}}$ and is already visible at $30 \mathrm{~K}$. As the sample temperature is lowered, small resonances emerge which correspond to the CF satellites (arrows). The spectra have been normalized at $V=-80 \mathrm{meV}$, which is indicated by the purple dashed line. Reprinted with permission from [115].

The temperature-dependent STS data show the emergence of a substructure at sufficiently low temperatures. The resonances surrounding the Fermi surface are the CF satellites of the Kondo resonance.

Realizing a two-atom quantum critical scenario. In 2011, STS was also used to investigate quantum criticality for Co atoms located on an $\mathrm{Au}(111)$ surface [116]. The most fundamental system obeying quantum criticality contains two magnetic impurities, where the distance between the magnetic moments is the tuning parameter. By attaching one magnetic Co atom to the STM tip and measuring STS on another Co atom placed on the $\mathrm{Au}(111)$ surface, the most fundamental, yet tunable quantum critical system is realized, see Fig. 4.12. By changing the vertical distance between the two atoms, the coupling strength $\Delta$ is smoothly tuned. If the coupling is weak (large vertical distance), a Fano resonance is observed, similar to the measurements shown in Fig. 4.10. In a two-atom system CF satellites are not expected because the point group symmetry remains unchanged with respect to the impurity case. By tuning the coupling strength, the system can be pushed into a quantum critical regime, which leads to a reduction of the resonance amplitude, a signature of unconventional quantum criticality. 

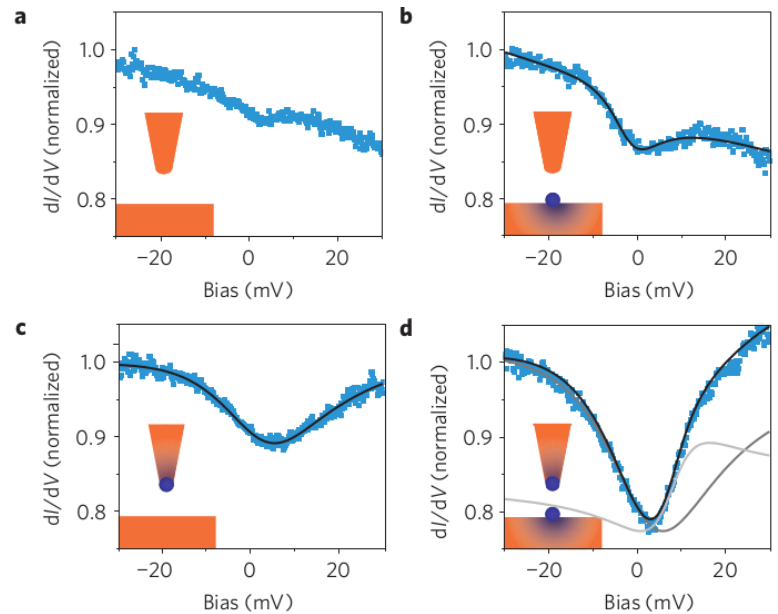

Figure 4.12.: Differential conductance data for different configurations with magnetic moments is displayed. (a) A spectrum of an $\mathrm{Au}$ tip on a clean $\mathrm{Au}(111)$ surface is displayed, as it shows no resonance (blue dots). (b) A spectrum taken on a Co atom located on the $\mathrm{Au}(111)$ surface induces a Kondo resonance, which is visible in the $\mathrm{d} I / \mathrm{d} V$ curve. The width of the resonance determines the effective Kondo temperature, which in this case is approximately $80 \mathrm{~K}$. (c) A similar $\mathrm{d} I / \mathrm{d} V$ curve is observed if a Co atom is attached to the tip instead of to the $\mathrm{Au}(111)$ surface. (d) If a Co atom is located on the tip as well as on the $\mathrm{Au}(111)$ surface, the resonance at $E_{\mathrm{F}}$ is strongly enhanced. The dark gray line indicates the resonance of the tip atom, displayed in (c) and the light gray curve is a fitted Fano resonance. The two contributions add up to the experimental observation (black line). Reprinted with permission from [116]. 


\subsubsection{Photoemission spectroscopy}

A commonly used ansatz to investigate band structures in solids are photoemission experiments. An intense UV or X-ray light pulse is used to photoexcite electrons into the surrounding vacuum. The photon energy $h \nu$ sets a hard limit to the energetically lowest lying bands accessible. For an electron to be ejected into the vacuum, the binding energy $E_{4 \mathrm{f}}$ (e.g. of an electron in a $4 \mathrm{f}$ shell) as well as the work function $\Phi$ have to be overcome. The kinetic energy $E_{\text {kin }}$ of the ejected electrons is given by the excess energy:

$$
E_{\text {kin }}=h \nu-E_{4 \mathrm{f}}-\Phi
$$

By detecting the photoexcited electrons, the DoS is directly monitored as function of the binding energy $E_{4 \mathrm{f}}$. In angle-resolved photoemission spectroscopy the full band structure can be reconstructed by measuring the angle as well as the energy of the ejected electrons [117]. Photoemission experiments are especially powerful thanks to the compatibility with femtosecond time resolution. However, some severe experimental restrictions in photoemission experiments hinder the investigation of strong correlation effects and other Fermi-surface instability phenomena. For instance, photoemission experiments have to be carried out in ultra-high vacuum and they are furthermore not accomplishable with magnetic bias fields. Furthermore, the electronic structure of the observed systems are heavily perturbed by the ionizing radiation, which is not optimal for investigating fragile Fermi-surface instabilities. Furthermore, pulsed light sources (e.g. from synchrotrons, lasers, free-electron lasers) are often used to reach sufficiently high fluences to extract as many electrons as possible. However, the pulse duration intrinsically limits the energy resolution as time and frequency are interconnected, see also chapter 5. In fact, it is a challenging task to reach meV energy resolution in photoemission experiments. Furthermore, this time-resolved method does not really allow us to investigate recombination processes of the excited electrons as they are extracted and removed from the ionized system. The high flux has the further disadvantage that the sample is strongly heated by the light pulse, which makes low temperature measurements (e.g. $T<15 \mathrm{~K}$ ) extremely challenging [36].

Despite the numerous experimental challenges, $\mathrm{CeCu}_{6}$ was investigated using high-resolution photoemission spectroscopy [118]. The temperature-dependent Kondo resonance at the Fermi energy was resolved as well as the onset of the first CF peak [36, 92. Remarkably, the Kondo spectral weight was observed for temperatures as large as $60 \mathrm{~K}$. Compared to the temperatures used to investigate Kondo physics in transport experiments, $60 \mathrm{~K}$ is a huge temperature and indeed well above the actual Kondo temperature of the system. This remarkable temperature behavior indicates that experiments involving photons are extremely sensitive to Kondo physics. Furthermore, systems even far from the QCP exhibit a ,Kondo-like‘ spin correlation, which contains information about the effective Kondo 
temperature $T_{\mathrm{K}}^{*}$, but it is unrelated to the heavy-fermion quasiparticle weight [36]. This aspect is further discussed and experimentally confirmed in section 7.1.2.

In contrast to photoemission experiments, with $\mathrm{THz}$ radiation the electrons are only stirred up slightly and remain within the system. For sufficiently low photon energies the electrons trapped in the Kondo singlets are excited just above the Fermi surface. As discussed in section 2.6, a small phase space strongly suppresses scattering processes, which potentially cause a loss of coherence. Because the phase space is kept sufficiently small with $\mathrm{THz}$ radiation (low photon energy), coherence is expected to be preserved for several picoseconds. Indeed, the intrinsic and coherent relaxation process of a heavy-fermion ground state can be phase-coherently detected on the picosecond time scale, as demonstrated in chapter 6.

Over the past three decades, many experiments in the $\mathrm{THz}$ range have been carried out on heavy-fermion systems [37, 109]. Their aim is mainly to characterize the equilibrium heavy-fermion state in the $\mathrm{GHz}$ and $\mathrm{THz}$ range. High-resolution electrodynamic response revealed that the Drude-Lorentz model seems to be also valid for heavy-fermion compounds 119 124]. The Drude-Lorentz model predicts that the width of the Drude conductivity peak scales inversely with the effective mass $m^{*}$, see section 5.8. Hence, for heavy-fermion systems this width of the Drude conductivity is expected to be in the $\mathrm{THz}$ or even $\mathrm{GHz}$ range [125. Because of the high energy resolution required, most experiments are carried out with narrow-band continuous-wave light sources. However, in these types of experiments no information about the intrinsic time scale can be gained. Only very recently, first time-resolved experiments were carried out on various heavy-fermion and Kondo-lattice systems [126-130]. The experiments shown in this thesis reveal the phase-coherent dynamics of heavy fermions (see chapter 6) and addresses the question, whether the fundamental concept of quasiparticles is still valid near unconventional QCP (see chapter 7). 



\section{THz time-domain spectroscopy}

Terahertz time-domain spectroscopy (THz-TDS) was developed in the 1980s and has strongly evolved since then. The key idea of THz-TDS is to acquire the signal temporally resolved and phase-sensitively [131-133. Hence, by Fourier transforming the $\mathrm{THz}$ pulse, also the spectral information can be accessed. $\mathrm{THz}$ radiation (T-rays) has a characteristic photon energy of just a few meV which is approximately three orders of magnitude lower than the photon energy of visible light. In the electromagnetic spectrum photons with meV energies can be found in the far-infrared regime. The corresponding frequency range is defined to be between 0.1 and $10 \mathrm{THz}$ which corresponds to photon energies between 0.4 and $41 \mathrm{meV}$.

With THz-TDS, not only the spectral information (with decent energy resolution) but also the time-resolved signal is acquired, which is often beneficial for the understanding of fundamental processes in condensed matter physics. The applicability of $\mathrm{THz}$ radiation ranges from detecting low-energy excitations in solids (phonons, magnons, excitons), to the detection of donor and acceptor levels in doped semiconductors. T-rays can be further used as a molecular characterization tool, since most molecules have eigenmodes in the lower $\mathrm{THz}$ range and hence strongly absorb well-defined $\mathrm{THz}$ frequencies. Already the slightest chemical modifications lead to drastic changes of its characteristic fingerprint. Due to the low photon energy of the radiation, also biological systems can be investigated with this non-ionizing radiation. For examples cells affected by cancer have an entirely different $\mathrm{THz}$ fingerprint compared to healthy cells. The non-ionizing nature of T-rays is the major reason why this technology is also widely used in security applications, e.g. at airports, as it is not considered be harmful to health.

\subsection{Overview}

Recent technological advances allow for strong $\mathrm{THz}$ sources even as table-top setups. Continuous $\mathrm{THz}$ wave sources have a narrow bandwidth as well as average power outputs as high as several $100 \mathrm{~mW} 132$. In contrast, pulsed $\mathrm{THz}$ sources are spectrally broad and have a much larger peak power, while their average output power remains low. High-intensity electromagnetic pulses can not only be used to perform spectroscopy measurements (magnons, electromagnons, phonons, excitons etc.), but can also be used to induce phase transitions by $\mathrm{THz}$ photodoping 134 139. THz radiation was also used in picosecond switching experiments in solids 140. 
The $\mathrm{THz}$ frequency range is located in the electromagnetic spectrum between the optical frequencies $\left(\sim 10^{15} \mathrm{~Hz}\right)$ and the electronically accessible frequencies (up to $\sim 10^{11} \mathrm{~Hz}$ ). This ,special ${ }^{6}$ location leads to some particular properties which are characteristic for T-rays. The most conspicuous features concerning metals are the following:

- Conducting surfaces are highly reflective in the $\mathrm{THz}$ range.

- The penetration depth $\delta$ for $\mathrm{THz}$ radiation into a conducting medium is of the order of a few nanometers.

These two facts make the applicability of $\mathrm{THz}$ radiation to metallic systems a lot more complex, see section 5.3. The skin depth $\delta$, i.e. the length scale of the exponential damping of an electromagnetic transient in a medium, can be directly derived from the Maxwell's equations [132]:

$$
\delta=\sqrt{\frac{2}{\omega \mu \sigma}},
$$

where $\omega$ is the frequency of the light transient, $\mu$ denotes the magnetic permeability and $\sigma$ the electrical DC conductivity. In good insulators, the electrical conductivity $\sigma$ is close to 0 , leading to a diverging $\delta$ and to a virtually undamped light transient. However, the penetration depth $\delta$ for light transients with frequency $1 \mathrm{TH}_{2}^{1}$ into a metal (e.g. $\mathrm{Cu}$ ) is of the order of $70 \mathrm{~nm}$, a vanishingly small distance compared to the wavelength of the transient itself ${ }^{2}$ Consequently, only metallic thin films with a thickness of the order of $\delta$ or less can be accessed in transmission geometry.

In order to investigate metallic single crystals, experiments in the $\mathrm{THz}$ range have to be performed in the experimentally more challenging reflection geometry. Note the fact that the response signal of metals is typically strongly dominated by the vast amount of conduction electrons. Hence, monitoring effects that only involve a minority of electrons can be particularly challenging. An example of such a system is the Kondo effect, where the major interest is in the small fraction of electrons involved in the Kondo screening. Hence, only a minor alteration of the dominating conduction electron signal is expected at first glimpse.

However, the author demonstrated the fundamentally different dynamics of excited strongly correlated states with respect to the (weakly correlated) metallic response in the Kondo-lattice systems, which allows us to temporally separate the two contributions. The tiny width of the Kondo spectral weight (given by the effective Kondo temperature $T_{\mathrm{K}}^{*}$ ) leads to immensely long lifetimes of several ps, compared to optical excitations which last only for a few fs or less. These long lifetimes of the excited ground state makes Kondo-lattice systems, despite their complexity, appealing for time-resolved studies. It turns out that the time delay for many Kondo-lattice systems $\left(T_{\mathrm{K}}^{*} \sim 10 \mathrm{~K}\right.$, see [67]) is large enough to temporally separate the instantaneous conduction electron response from the Kondo response signal,

${ }^{1} \omega=2 \pi \nu=2 \pi \cdot 1 \mathrm{THz}$

${ }^{2} \lambda \approx 0.3 \mathrm{~mm}$ for $1 \mathrm{THz}$ in free space 
see chapter 6. This fact of strong correlation dynamics helps to improve also the sensitivity drastically since the strong correlation features are accessed virtually background-free in the time domain.

This chapter is dedicated to giving an overview over the broad field of T-ray optics, their properties as well as an insight into the experimental challenges faced by the author while performing the THz-TDS experiments on Kondo lattices. Thanks to the phase-sensitive detection scheme in THz-TDS, the information gained in measurements is more exhaustive and more revealing than e.g. in $\mathrm{THz}$ frequency-domain spectroscopy (THz-FDS) [132. However, processing the raw data acquired in the time domain requires a more demanding analysis which is explained in this chapter as well [131, 133.

\subsection{Coherent terahertz radiation and their outstanding properties}

In order to generate temporally short $\mathrm{THz}$ pulses (few cycles or even less), often strong and pulsed light source $\$^{3}$ emitting in the near-infrared (NIR) are converted. These NIR pulses can be converted to T-rays by using nonlinear conversion processes. In a descriptive manner, these processes are called frequency-down conversions 132 . Because the $\mathrm{THz}$ range remains more challenging to access than other parts of the electromagnetic spectrum, it is also often referred to as the $\mathrm{THz}$ gap. Recent technological developments, though, are responsible for an impressive progress in the field and enabled powerful table-top $\mathrm{THz}$ sources, even with the capability of pulse tailoring. However, the experimental work to build and operate these devices remains challenging and requires lots of experience. Furthermore, most known optical elements do not exist yet for the $\mathrm{THz}$ range or they are currently being developed.

An omnipresent source for (incoherent) THz radiation is black-body radiation of room temperature objects. The emitted radiation of a black body at a temperature $T$ is given by the Planck law. The black-body spectrum has a maximum at the photon frequency specified by:

$$
\nu_{\max }=58.8 \mathrm{GHz} / \mathrm{K} \cdot T .
$$

Therefore, room temperature objects are good THz emitters. However, the emitted light is temporally and spatially incoherent. In order to generate a coherent $\mathrm{THz}$ signal, either a $\mathrm{THz}$ cavity has to be built (quantum-cascade lasers [141]) or alternatively an ultrashort excitation pulse (much shorter than the periodicity of the $\mathrm{THz}$ pulse itself) can be used to stimulate a $\mathrm{THz}$ emitter. For instance in photoconductive switches, a femtosecond laser pulse is used to

\footnotetext{
${ }^{3}$ e.g.: Ti:Sapphire lasers are emitting at $\lambda=800 \mathrm{~nm}$. The pulse duration of commercially available lasers can be as short as a few femtoseconds.
} 
generate photoexcited charge carriers which then hot-wire a biased antenna [132]. Alternatively, high-intensity pulses can be used to trigger a nonlinear material response, which subsequently emit pulsed T-ray transients. Commonly used $\mathrm{THz}$ emitters are non-centrosymmetric crystals which are pumped with high-intensity femtosecond laser pulses. The coherent $\mathrm{THz}$ pulses are generated by a nonlinear wave-mixing progress within the crystal, see section 5.4. Due to the complexity of the THz-TDS setups, most $\mathrm{THz}$ experiments are restricted to room temperature and transmission geometry.

Due to the short duration of coherent $\mathrm{THz}$ pulses with respect to the wavelength (a few-cycle pulse), the spectrum of such pulses spans a wide range. Because of the multichromatic property of the T-ray bursts, lenses are not suitable for focusing the beam due to dispersion and aberration effects. Hence, focusing T-rays is often realized by curved reflection optics such as parabolic mirrors, even though they introduce aberration. Nevertheless, focusing few-cycle $\mathrm{THz}$ pulses remains difficult due to paraxial approximation issues 4 In THz-TDS the electric field is directly monitored as function of time. In order to be able do this, a fast and phase-sensitive detection scheme has to be implemented, see section 5.5. To extract spectral information from the time domain, the complex Fourier transform has to be applied.

\subsection{Experimental setup}

A major part of the author's doctoral thesis was to design, build and develop a low-temperature terahertz time-domain spectrometer which can be used either in transmission mode (for semiconductors and insulators) as well as in reflection mode (for metallic systems such as Kondo lattices). The author used a $1.6 \mathrm{~W}$, $130 \mathrm{fs}, 800 \mathrm{~nm}$ beam from a Ti:Sapphire laser which has a repetition rate of $1 \mathrm{kHz}$. Furthermore, a Janis SVT-400 flow cryostat was used in order to tune the sample temperature between room temperature $(300 \mathrm{~K})$ and the superfluid liquid helium temperature $(1.8 \mathrm{~K})$. In order to sample the terahertz pulse in the time domain, a mechanical Physik Instrumente M-414.3PD translation stage is used as a delay line. It has a unidirectional repeatability precision of $1 \mu \mathrm{m}$ which corresponds to a precision of $3 \mathrm{fs}$. The delay line has a working distance of $30 \mathrm{~cm}$. When using the stage as a four-way delay line, a maximum light path difference of $1.2 \mathrm{~m}$ can be achieved. This corresponds to a maximum time-trace length of $4 \mathrm{ns,} \mathrm{which}$ corresponds to a theoretically achievable frequency resolution of below $1 \mathrm{GHz}$ or $4 \mu \mathrm{eV}$. However, trivial etalon effects have to be taken into account for long time traces, see also appendix D.2.

The THz-TDS setup shown in Fig. 5.1 allows us to measure $\mathrm{THz}$ pulses time-resolved. Hence, a single $\mathrm{THz}$ pulse reflected (or transmitted) by a sample allows us to investigate the properties of the specimen in the $\mathrm{THz}$ range. THz-TDS

\footnotetext{
${ }^{4}$ For example, avoiding astigmatism (i.e. the different focusing properties along different spatial axes) in the $\mathrm{THz}$ regime is challenging.
} 
allows us to investigate specific absorptions of samples, which might change as external parameters are altered, such as the temperature or the magnetic field applied.

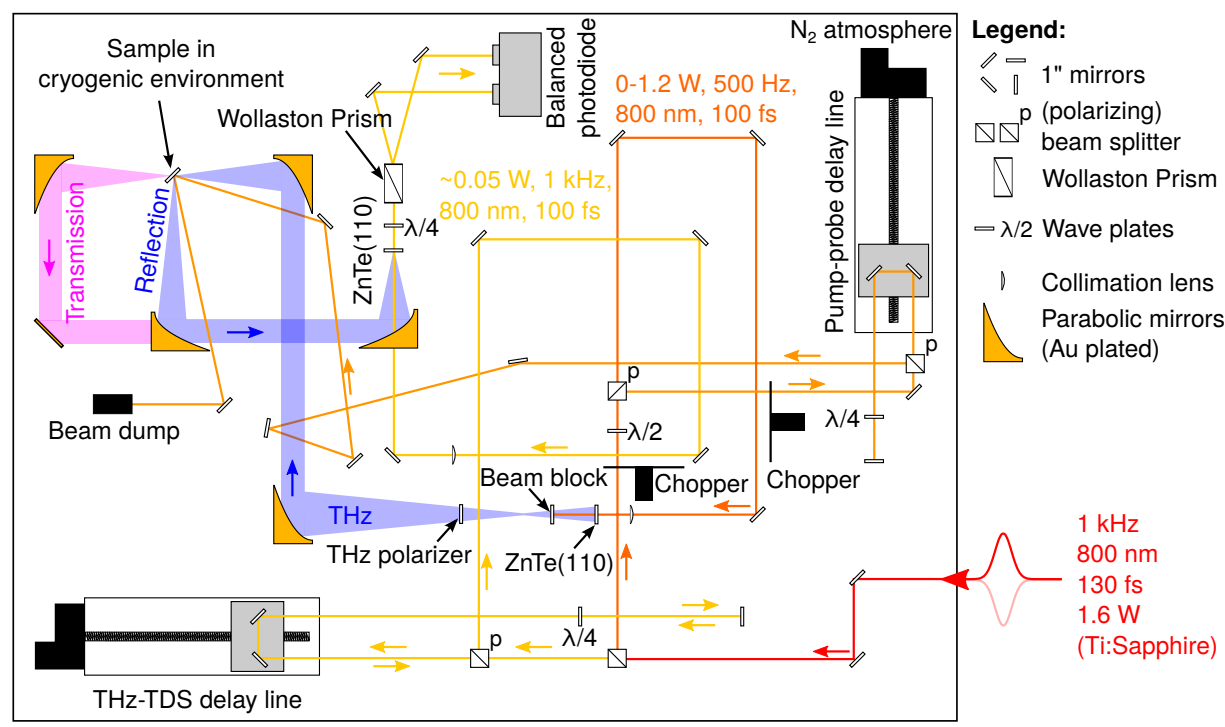

Figure 5.1.: Sketch of the experimental setup. The incident beam is split three times. The two most intense parts are used to pump the sample (either with $\mathrm{THz}$ or with optical frequencies) as well as to generate T-rays in a $\mathrm{ZnTe}(110)$ single crystal. The weakest NIR pulse is used to probe the T-rays in an electro-optical sampling crystal. By tuning the half-wave plate (located behind the first chopper), the power ratio between the first and second NIR pulse can be selected. Hence, the device can run in three modes: ,pump-probe' (two pulses, one much stronger than the other), ,double-pulse' (two equally strong pulses) and, $\mathrm{THz}$ spectroscopy (only the $\mathrm{THz}$ spectroscopy branch) mode. For more details, see text.

Alternatively to the changes of the ambient conditions, interesting insight can often be gained when the sample is perturbed only for a short amount of time (on the fs or ps time scale), as the system relaxes from the excited state back into its ground state. An ideal tool to perturb the system are high-intensity laser pulses, which allow to drive the sample out of equilibrium on a femtosecond time scale. The relaxation mechanisms can be monitored by performing THz-TDS measurements as function of the relative delay time $\tau$ between the pump beam and the $\mathrm{THz}$ probe beam.

The author's setup is also designed to perform these types of optical pump- $\mathrm{THz}$ probe experiments. Therefore, the NIR pulse used to generate the T-rays is further split. With the stronger part of this pulse, the system can be either perturbed using a NIR or a second T-ray pulse. The T-rays are generated using the weaker 
part of the NIR pump pulse. In order to perform these double-pulse experiments, a second delay line is required, defining the delay time $\tau$. The author used a Physik Instrumente M-404.42S stepper delay line (with a unidirectional resolution of $1 \mu \mathrm{m}$ ) with a length of $10 \mathrm{~cm}$, which corresponds to a dynamical range of $1.3 \mathrm{~ns}$ (four-way delay line). Note that for these types of experiments a double-chopping scheme has to be employed to enhance sensitivity. The frequency of the second chopper has to be far lower than the first chopper frequency. An upper limit of the second chopper frequency is given by the integration time of the lock-in amplifier used to acquire the $\mathrm{THz}$ pulse. With a second lock-in amplifier, which is locked to the second chopper, the alterations of the $\mathrm{THz}$ pulse caused by the optical perturbation can be extracted.

Furthermore, the entire setup was designed and built on a $120 \times 90 \mathrm{~cm}^{2}$ baseplate. The compactness of the spectrometer guarantees high flexibility: It can be easily transported and, for example, be used in combination with a high repetition rate Ti:Sapphire oscillator. The controller softwares and graphical user interfaces were written by the author, who used LabVIEW for the data acquisition and the MATLAB software environment for the detailed evaluation.

\subsubsection{In detail description of the $\mathrm{THz}$ setup}

In order to do THz-TDS, the pump laser entering the $\mathrm{THz}$ spectrometer has to be split into two beams, a high-intensity part for the $\mathrm{THz}$ optical rectification process (see section 5.4) and a weak intensity part for the electro-optical sampling (see section 5.5). The THz-TDS setup is already a ,pump-probe-like setup, where the pump pulse is guided onto the generation crystal. The probe pulse is guided, simultaneously and parallelly with the T-ray beam onto the detection crystal. By temporally shifting the pump and the probe beam, the $\mathrm{THz}$ pulse can be scanned in the time domain, resolving the phase information.

In order to improve the signal-to-noise ratio, the electro-optical sampling is measured with twice the frequency of the generated $\mathrm{THz}$ pulses. This ,on-off' scheme allows us to subtract the background signal for every T-ray pulse. Subtracting T-ray background is crucial, since the black-body radiation of objects at room temperature is also in the $\mathrm{THz}$ range. A highly improved sensitivity is achieved using a digital SRS-830 lock-in amplifier to subtract the reference pulse by pulse. Fig. 5.2 illustrates a time interval of the signal acquisition. 


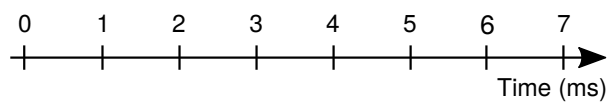

a

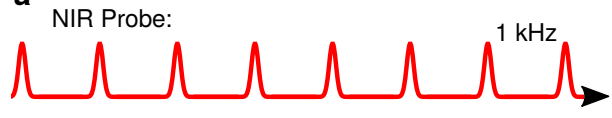

b

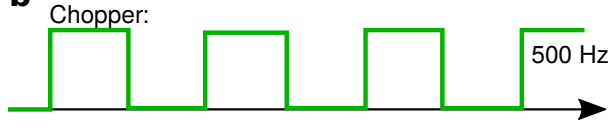

C NIR Pump/ THz generation:

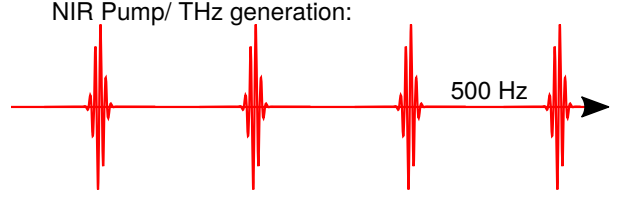

d

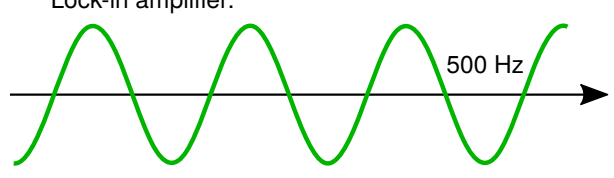

Figure 5.2: Time sequence of $\mathrm{THz}-\mathrm{TDS}$ setup. (a) The $1 \mathrm{kHz}$ Ti:Sapphire laser emits every ms a $130 \mathrm{fs}$ NIR pulse, which can be used for sampling the $\mathrm{THz}$ beam. (b) A mechanical chopper is used to block every second pulse in order to generate $\mathrm{THz}$ with a repetition rate of $500 \mathrm{~Hz}$. (c) The $\mathrm{THz}$ radiation is generated with half the sampling frequency (which is $1 \mathrm{kHz}$ ) to improve the setup sensitivity by subtracting the background. (d) A digital SRS-830 lock-in amplifier is operated at $500 \mathrm{~Hz}$, in order to subtract the background for every T-ray pulse. This time sequence is repeated for every delay time between the $\mathrm{THz}$ and the sampling beam in order to scan the pulse in the time domain.

Pump path. The high-intensity part of the laser beam is guided onto a $\mathrm{ZnTe}(110)$ single crystal for the $\mathrm{THz}$ generation, see section 5.4. Since a few-cycle $\mathrm{THz}$ pulse is generated, the spectral width of the pulse is two orders of magnitude wide: from 3 to $100 \mathrm{~cm}^{-1}$ or between 0.1 and $3 \mathrm{THz}$, see Fig. 5.4. Hence, the spherical and spatial aberrations of ordinary lenses are too large and therefore parabolic reflective optics (Au coated parabolic mirrors) are used to collimate and focus the $\mathrm{THz}$ pulse. One has to further take into account that the Rayleigh length $z_{\text {Rayleigh }}$ scales inversely proportional to the wavelength:

$$
z_{\text {Rayleigh }}=\frac{n \pi w_{0}^{2}}{\lambda^{0}}
$$

where $n$ is the refractive index, $w_{0}$ the beam waist and $\lambda_{0}$ the wavelength. The Rayleigh length specifies the divergence of the Gaussian beam. Furthermore, the opening angle $\theta_{\text {div }}$ of the Gaussian is given by Eq. 5.4 .

$$
\theta_{\text {div }} \approx \frac{2 w_{0}}{z_{\text {Rayleigh }}}
$$

Because the wavelength of $\mathrm{THz}$ radiation is in the order of $\mathrm{mm}$, it is a lot more divergent than NIR radiation with the same beam waist.

In order to generate $\mathrm{THz}$, the NIR pump beam is focused onto the focus of the first parabolic mirror, see also Fig. 5.2. In order not to damage the optical rectification 
crystal and not to have any plasma generation inside the setup, the generation crystal as well as the high-density polyethylene (HDPE) beam blocker for the NIR pump beam are placed well in front of the focus [142]. To collect and collimate the $\mathrm{THz}$ radiation, a two-inch gold-plated parabolic mirror is used. The collimated T-ray beam has a diameter of $D_{\mathrm{THz}} \approx 25 \mathrm{~mm}$. A second parabolic mirror is used to focus the collimated beam onto the sample located inside the cryostat, in order to reach higher electromagnetic field strengths on the specimen, see section 5.10 .

In reflection mode, the sample has to be aligned to reflect the incident $\mathrm{THz}$ radiation into the third parabolic mirror, which is located at 90 degrees relative to the incoming $\mathrm{T}$-ray light path. It collects the reflected $\mathrm{THz}$ radiation from the sample and sends it re-collimated to the fourth and last parabolic mirror. The third parabolic mirror has to be removed in order to perform transmission experiments. In transmission mode, the transmitted $\mathrm{THz}$ radiation is being collected and re-collimated by the parabolic mirror located behind the sample. With a planar two-inch gold mirror the parallel beam is guided onto the fourth parabolic mirror.

The fourth and last parabolic mirror focuses the $\mathrm{THz}$ radiation onto the electro-optical detection crystal. This last parabolic mirror has a pin hole (pin-hole diameter: $D_{\text {pinhole }}=1.8 \mathrm{~mm}$ ) which is used to inject the parallelly propagating probe beam. Due to the pin hole, only a small amount of $\mathrm{THz}$ signal is lost 5

$$
\frac{E_{\mathrm{THz}}^{\text {pin hole }}}{E_{\mathrm{THz}}^{0}}=\frac{\pi\left(\frac{D_{\text {pin hole }}}{2}\right)^{2}}{\pi\left(\frac{D_{\mathrm{THz}}}{2}\right)^{2}} \approx 14 \% .
$$

In order to align the sample in reflection geometry, a second alignment diode path is used, hitting the sample under a smaller angle of incidence. With two distant alignment irises the sample can be oriented an positioned very sensitively. However, this alignment method is less precise than an interferometric positioner. Nevertheless, precisions of the order of $10 \mu \mathrm{m}$, which corresponds to $30 \mathrm{fs}$, can be achieved.

Probe path. The low-intensity part of the NIR pulse is guided onto the four-way mechanical delay stage M-414.3PD from Physik Instrumente. The step size defines the temporal resolution, and therefore the highest detectable frequency. The upper limit for highest possible $\mathrm{THz}$ frequency generated is given by the transverse-optical (TO) phonon mode of the generation crystal. In the case of ZnTe, the TO phonon mode is reported to be at $5.3 \mathrm{THz}$ [143, 144. To detect frequencies of up to $5 \mathrm{THz}$, the stepsize in probing light path has to be $60 \mu \mathrm{m}$ or less, see section 5.9 . However, time traces are typically oversampled with a temporal resolution of $6 \mu \mathrm{m}$, which corresponds to a time step of $20 \mathrm{fs}$. After the mechanical delay line, the probe beam is then guided through the pin hole in the fourth parabolic mirror to collinearly propagate with the T-rays onto the detection crystal. The collinear incidence of the

\footnotetext{
${ }^{5}$ For this rough estimation, a flat-top $\mathrm{THz}$ beam profile is assumed.
} 
two beams is crucial in order to fulfill the phase-matching condition in $\mathrm{ZnTe}(110)$, see section 5.4 .

In the electro-optical sampling crystal, the electric field strength of T-ray pulse induces a birefringence, which is measured by this NIR sampling beam. The $\mathrm{THz}$-induced birefringence causes a change in the ellipticity of the probe beam, which can be measured using a quarter-wave plate and a Wollaston prism, which spatially separates s- and p-polarized light. Because of the quarter-wave plate, the difference between the two beams corresponds to the ellipticity of the the probe beam, which can be measured using a balanced photodiode. Further details of the detection are given in section 5.5

\subsection{Generation of $\mathrm{THz}$ pulses}

A wide range of $\mathrm{THz}$ sources is nowadays available. Depending on the specific application, one or the other property is preferred. For THz-TDS a broadband source is favorable, as a wide spectral range can be captured by a single time trace of the $\mathrm{THz}$ pulse. A narrow-band source on the other hand can be used to very selectively excite a specimen. A brief overview over the most common T-ray sources is given in Table 5.1.

\begin{tabular}{c|l|l|c} 
& Source & Generation mechanism & \\
\hline 1 & $\begin{array}{l}\text { Photoconductive } \\
\text { antenna }\end{array}$ & $\begin{array}{l}\text { fs laser pulse induces free charge carriers } \\
\text { and short-circuits the biased antenna }\end{array}$ & $\mathrm{B}$ \\
\hline 2 & Optical rectification* & $\begin{array}{l}\text { fs laser pulse induces nonlinear response } \\
\text { (difference frequency) }\end{array}$ & $\mathrm{B}$ \\
\hline 3 & Plasma generation & $\begin{array}{l}\text { fs laser pulse generates plasma, } \\
\text { which breaks inversion symmetry and } \\
\text { allows the difference frequency process }\end{array}$ & $\mathrm{B}$ \\
\hline 4 & $\begin{array}{l}\text { Synchrotrons } \\
\text { and FELs }\end{array}$ & $\begin{array}{l}\text { Deflected, relativistic electrons } \\
\text { emit bremsstrahlung }\end{array}$ & $\mathrm{B}$ \\
\hline \hline 5 & $\begin{array}{l}\text { Quasi-phase-matching } \\
\text { crystals }\end{array}$ & $\begin{array}{l}\text { Phase matching over long distance } \\
\text { achieved by periodically poling }\end{array}$ & $\mathrm{N}$ \\
\hline 6 & Quantum-cascade lasers & Intraband transition in solids & $\mathrm{N}$ \\
\hline 7 & $\begin{array}{l}\text { Backward-wave } \\
\text { oscillators }\end{array}$ & $\begin{array}{l}\text { continuous-wave THz is generated } \\
\text { using an electron beam }\end{array}$ & $\mathrm{N}$ \\
\hline
\end{tabular}

Table 5.1.: Overview of commonly used $\mathrm{THz}$ sources 132. The sources 1-4 are so-called broadband $\mathrm{THz}$ sources (indicated as , $\mathrm{B}^{*}$ ) used for time-resolved measurements. The spectral width is crucial to be able to temporally compress the pulse. On the other hand, the sources 5-7 are more narrow-band sources (indicated as , $\mathrm{N}^{\natural}$ ), used to perform high energy-resolved spectroscopy. Why narrow-band sources cannot be temporally compressed is explained in section 5.6 . For the experiments presented in this thesis, the optical rectification process $\left(\right.$ marked with ${ }^{*}$ ) was used to generate the $\mathrm{THz}$ pulses, see also section 5.4.1. 
In Table 5.1, the T-ray sources 1 to 4 are broadband sources, whereas 5 to 7 are so-called narrow-band T-ray emitters. The narrow-band sources achieve high average output power, whereas the pulsed source reach much higher peak intensities. The most commonly used methods for generating short $\mathrm{THz}$ pulses in a table-top THz-TDS are 1 and 2, where a frequency down-conversion is realized by a two-wave mixing. Nonlinear crystals with a high damaging threshold are often used (method 2), since they do not have to be biased with a high voltage, in contrast to photoconductive antennas (method 1). For all these reasons, the author used the optical rectification process in $\mathrm{ZnTe}$, in order to generate coherent $\mathrm{THz}$ for his Kondo-lattice experiments, see section 5.4.2. A priori, the generation crystals have to fulfill the following aspects to qualify as good $\mathrm{THz}$ emitters:

Transparency. The crystal has to be sufficiently transparent for T-rays and the pump wavelength, which is typically in the NIR. Note that T-ray transparency is mainly affected by TO phonon modes, which are often strong absorbers in the $\mathrm{THz}$ range.

Broken spatial inversion symmetry. THz radiation is generated by difference frequency generation, which is a two wave-mixing process. However, second-order terms in the susceptibility $\chi$ are only allowed in non-centrosymmetric structures.

Phase matching (velocity matching). A further restriction for effective T-ray generation is the constructive interference of $\mathrm{THz}$ radiation generated in different layers of the crystal. To fulfill this constructive interference condition, the generated $\mathrm{THz}$ source as well as the pump beam must travel with equal velocity through the generation crystal. If the group velocity of the pump beam equals the phase velocity of the $\mathrm{THz}$ pulse, the phase-matching condition is fully satisfied. Phase matching sometimes is also realized by phase-front tilting (e.g. in $\mathrm{LiNbO}_{3}$ ), leading to non-collinear pump and T-ray propagation. This pump scheme is called quasi-phase-matching scheme, as the different propagation speeds can be geometrically corrected [145, 146].

A detailed mathematical explanation for the $\mathrm{THz}$ generation description is given in section 5.4.1. It turns out that ZnTe single crystals oriented along the (110) direction are good $\mathrm{THz}$ emitters. However, the phase-matching condition is only fulfilled for a certain pump wavelength. For $\mathrm{ZnTe}(110)$, the T-rays are phase matched with $800 \mathrm{~nm}$, which is very convenient for generating $\mathrm{THz}$ using Ti:Sapphire lasers [147, 148. ZnTe has a zincblende crystal structure (space group F $\overline{4} 3 \mathrm{~m}$, no. 216), in which a two-photon difference frequency generation is allowed by symmetry. The conversion rate is given by the second-order susceptibility term, which is reported to be $d_{14}=3.9 \mathrm{pm} / \mathrm{V}$ in $\mathrm{ZnTe}(110)$ [147]. Furthermore, the temporal trace of the emitted $\mathrm{THz}$ pulse strongly depends on the spectral width of the pump laser as well as the crystal itself. ZnTe(110) crystals, in combination with a 130 fs Ti:Sapphire laser, are known to emit a few-cycle $\mathrm{THz}$ pulse with a central frequency of approximately $4 \mathrm{meV}$. The lower $\mathrm{THz}$ range is ideal for effectively 
perturbing the weakly bound Kondo singlets as the Kondo temperature is typically in the order of $10 \mathrm{~K}$ [67].

In the late 1990s, organic salts were discovered as promising high-field $\mathrm{THz}$ sources 149 151. Examples of organic THz sources are DAST ${ }^{6}$ DSTMS 7 and OH1 8 These organic crystals are particularly interesting because of their large nonlinear coefficients, which is in the range of $50 \mathrm{pm} / \mathrm{V}$ and therefore approximately one order of magnitude larger than for inorganic crystals. Unfortunately, the phase-matching condition for these organic crystals is typically fulfilled for pump wavelengths between $1.2-1.6 \mu \mathrm{m}$ [150. Hence, these crystals cannot be directly pumped using Ti:Sapphire lasers which emit light with a central wavelength of $800 \mathrm{~nm}$.

\subsubsection{Optical rectification process to generate $\mathrm{THz}$}

The $\mathrm{THz}$ generation process is triggered by the quadratically induced polarization of the generation crystal. Described by Eq. 5.6, the material characteristics are captured by the complex refractive index $\tilde{n}$ :

$$
\vec{P}=\chi \vec{E}(\omega) \quad, \quad \tilde{n}^{2}(\omega)=1+\chi(\omega)
$$

$\vec{P}$ is the light-induced polarization, $\vec{E}$ the incident electric field oscillating at frequency $\omega$ and $\chi$ the susceptibility tensor. The susceptibility tensor can be expanded into a Taylor series:

$$
\begin{aligned}
\chi & =\chi^{(1)}+\chi^{(2)}+\chi^{(3)}+\ldots \\
\text { i.e.: } \quad P_{i} & =\chi_{i j}^{(1)} E_{j}+\chi_{i j k}^{(2)} E_{i} E_{j}+\chi_{i j k l}^{(3)} E_{j} E_{k} E_{l}+\ldots
\end{aligned}
$$

where $\chi^{(n)}$ describes the $n$-th order response. Hence, high-intensity light pulses are required to induce a nonlinear response. An ideal source of high electromagnetic fields are Ti:sapphire lasers, which produce ultrashort light pulses, with up to tens of gigawatts of peak power. In the experimental setup built in the framework of this thesis, $130 \mathrm{fs}$ pulses, each with $1.6 \mathrm{~mJ}$ of energy, are used to illuminate a large-area $\mathrm{ZnTe}(110)$ generation crystal. The electric field intensity of NIR pulse is sufficiently large to induce reasonably higher-order material responses. Eq. 5.7 illustrates that the linearly induced polarization oscillates with the same frequency $\omega$ as the incident electric field. However, the second-order response will cause a light-induced polarization oscillating with twice the frequency of the incident light as well as with the difference of the two incident frequencies. A temporally short pulse is spectrally broad (see section 5.6), which means that a 130 fs laser pulse

\footnotetext{
${ }^{6} 4$-N,N-dimethylamino-4'-N'-methyl-stilbazolium tosylate

${ }^{7} 4$-N,N-dimethylamino-4'-N'-methyl-stilbazolium 2,4,6-trimethylbenzenesulfonate

82-[3-(4-hydroxystyryl-5.5-dimethylcyclohex-2-enylidene]malononitrile
} 
contains multiple frequencies. Hence, the difference frequency generated by a single short laser pulse is not vanishing, see Fig. 5.3 .

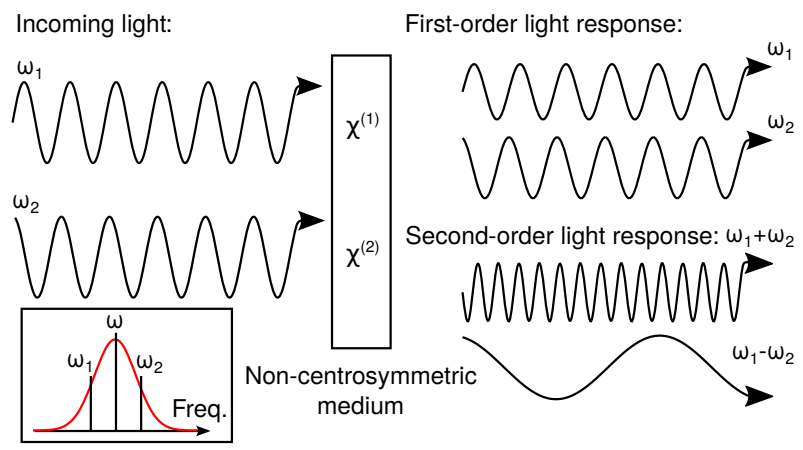

Figure 5.3.: Illustration of second-order wave-mixing processes. Two incident frequencies $\omega_{1}$ and $\omega_{2}$ on a non-centrosymmetric structure (and non-vanishing $\left.\chi^{(2)}\right)$ cause different types of responses. The two frequency components $\omega_{1}$ and $\omega_{2}$ might even originate from a single, spectrally broad pulse centered at $\omega$ (see inset box). The FWHM of the pulse spectrum allows us to estimate $\omega_{1}$ and $\omega_{2}$. In contrast, the first-order response does not affect the frequency of the transmitted wavelength.

The latter process only results in a non-vanishing frequency $\omega_{1}-\omega_{2}$ because of the broad spectrum of the temporally strongly confined pulses, see section 5.6. For a $130 \mathrm{fs}$ laser pulse, the difference frequency generated within a single pulse lies within the $\mathrm{THz}$ range of the electromagnetic spectrum. The T-rays can be separated from the fundamental pump beam and the second-harmonic response of the crystal by using selective filters. This process is also called optical rectification. Since the T-ray pulse is temporally confined to a few cycles, its THz spectrum is also spectrally broad. Hence, the highest possible $\mathrm{THz}$ frequency is given by the FWHM of the NIR pump pulse spectrum [152]. As a result, the highest possible $\mathrm{THz}$ frequency generated by a $130 \mathrm{fs}$ laser pulse is:

$$
\nu_{\max } \approx 3.4 \mathrm{THz}
$$

An analytical solution of the generated $\mathrm{THz}$ pulse was calculated by Schneider and co-workers [153. Their results are discussed in appendix A.1. Inorganic crystals are known for their high laser damage thresholds. Additionally, they are often perfectly phase matched with the output wavelength of Ti:Sapphire lasers $(800 \mathrm{~nm})$ and therefore, inorganic crystals are preferably used to generate pulses in the lower $\mathrm{THz}$ range (between 0.2 and $4 \mathrm{THz}$ ). Since the characteristic energy scales of most Kondo systems and heavy fermions is also in the lower $\mathrm{THz}$ range (see chapters 2 and 4, the author decided to perform the THz-TDS experiments using inorganic ZnTe(110) crystals. 


\subsubsection{Orientation-dependent optical rectification process in ZnTe}

ZnTe is an inorganic single crystal with a zincblende crystal structure (space group: $\mathrm{F} \overline{4} 3 \mathrm{~m}$ ). By exciting the correctly oriented ZnTe crystal with a $130 \mathrm{fs}$ laser pulse at $800 \mathrm{~nm}$, it emits a few cycle $\mathrm{THz}$ pulse with a central frequency of approximately $0.95 \mathrm{THz}$. The emitted T-ray pulse of the author's setup and its corresponding spectrum looks as depicted in Fig. 5.4 .

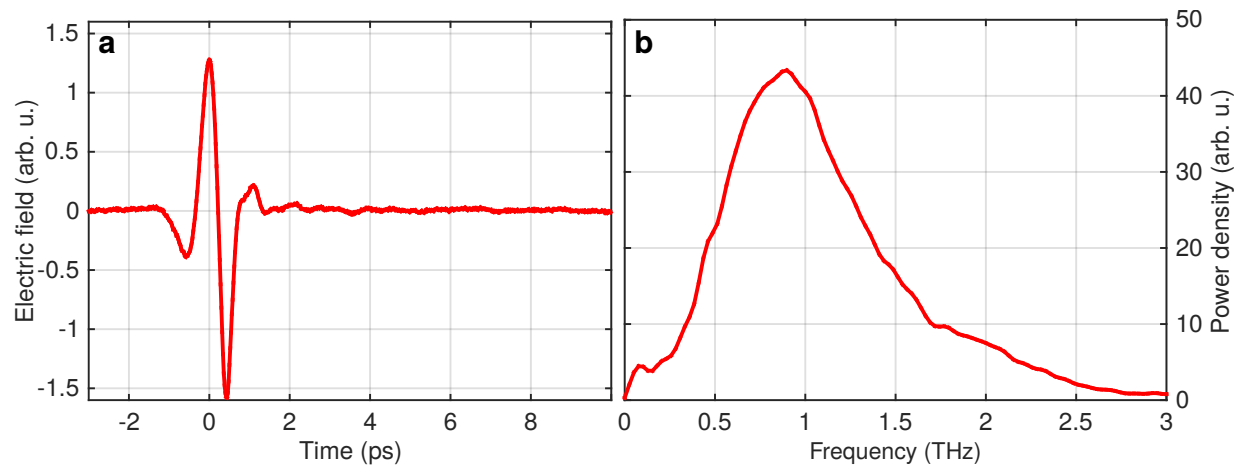

Figure 5.4.: (a) Time-domain signal emitted by ZnTe(110). A platinum mirror was placed inside the cryostat in order to reflect the incident $\mathrm{THz}$ radiation. (b) The corresponding power density of the time trace. The central frequency is approximately $0.95 \mathrm{THz}$ and the FWHM is $0.9 \mathrm{THz}$.

The correct orientation of the generation crystal is vital. The second-order term $\chi^{(2)}$ is a third-rank tensor, for which the following relation holds [132]:

$$
\chi_{i j k}^{(2)}=\chi_{i k j}^{(2)}
$$

Hence, $\chi^{(2)}$ can be projected onto the 3-by-6 matrix $d$, which is defined as follows [144:

$$
\left(\begin{array}{c}
P_{x} \\
P_{y} \\
P_{z}
\end{array}\right):=2 \epsilon_{0} d\left(\begin{array}{c}
E_{x}^{2} \\
E_{y}^{2} \\
E_{z}^{2} \\
2 E_{y} E_{z} \\
2 E_{z} E_{x} \\
2 E_{x} E_{y}
\end{array}\right) \quad, \quad d_{i l}=\frac{1}{2} \chi_{i j k}^{(2)}
$$

$$
i, j, k \in\{1,2,3\} \text { and } l \in\{1,2,3,4,5,6\} \text {, }
$$

where indices $l=1,2,3$ equal $(j, k)=(1,1),(2,2),(3,3), l=4$ corresponds to $(j, k)=(2,3),(3,2), l=5$ to $(j, k)=(1,3),(3,1)$ and $l=6$ to $(j, k)=(1,2),(2,1)$. 
For systems with the zincblende structure, the $d$ matrix has only three non-zero entries, which are furthermore identical $\left(d_{14}=d_{25}=d_{36}\right)$ :

$$
d=\left(\begin{array}{cccccc}
0 & 0 & 0 & d_{14} & 0 & 0 \\
0 & 0 & 0 & 0 & d_{14} & 0 \\
0 & 0 & 0 & 0 & 0 & d_{14}
\end{array}\right)
$$

The angular dependence of the generation process can be described by expressing the incident electric field in spherical coordinates:

$$
\begin{aligned}
& \left(\begin{array}{l}
P_{x} \\
P_{y} \\
P_{z}
\end{array}\right)=2 \epsilon_{0} d_{14} E_{0}^{2}\left(\begin{array}{llllll}
0 & 0 & 0 & 1 & 0 & 0 \\
0 & 0 & 0 & 0 & 1 & 0 \\
0 & 0 & 0 & 0 & 0 & 1
\end{array}\right)\left(\begin{array}{c}
\sin ^{2} \theta \cos ^{2} \phi \\
\sin ^{2} \theta \sin ^{2} \phi \\
\cos ^{2} \theta \\
2 \sin \theta \cos \theta \sin \phi \\
2 \sin \theta \cos \theta \cos \phi \\
2 \sin ^{2} \theta \sin \phi \cos \phi
\end{array}\right) \\
& \text { with } \vec{E}=E_{0}\left(\begin{array}{c}
\sin \theta \cos \phi \\
\sin \theta \sin \phi \\
\cos \theta
\end{array}\right)
\end{aligned}
$$

The emitted T-ray intensity is proportional to the quadratically light-induced polarization $|\vec{P}|^{2}$ :

$$
\begin{aligned}
I \propto|\vec{P}|^{2} & =\left(2 \epsilon_{0} d_{14} E_{0}^{2} \sin \theta\right)^{2}\left|\left(\begin{array}{c}
2 \sin \theta \cos \theta \sin \phi \\
2 \sin \theta \cos \theta \cos \phi \\
2 \sin ^{2} \theta \sin \phi \cos \phi
\end{array}\right)\right|^{2} \\
& =\left(2 \epsilon_{0} d_{14} E_{0}^{2} \sin \theta\right)^{2}\left(4 \cos ^{2} \theta+\sin ^{2} \theta \sin ^{2} 2 \phi\right)
\end{aligned}
$$

Fig. 5.5 shows the orientation-dependent $\mathrm{THz}$ emission of ZnTe. Hence, in optical rectification crystals with $\overline{4} 3 m$ symmetry, the (110) crystal orientation is the most efficient. 
a

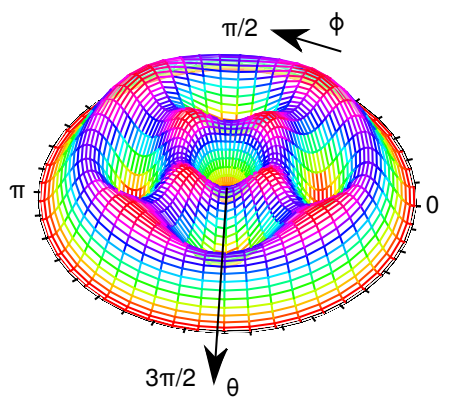

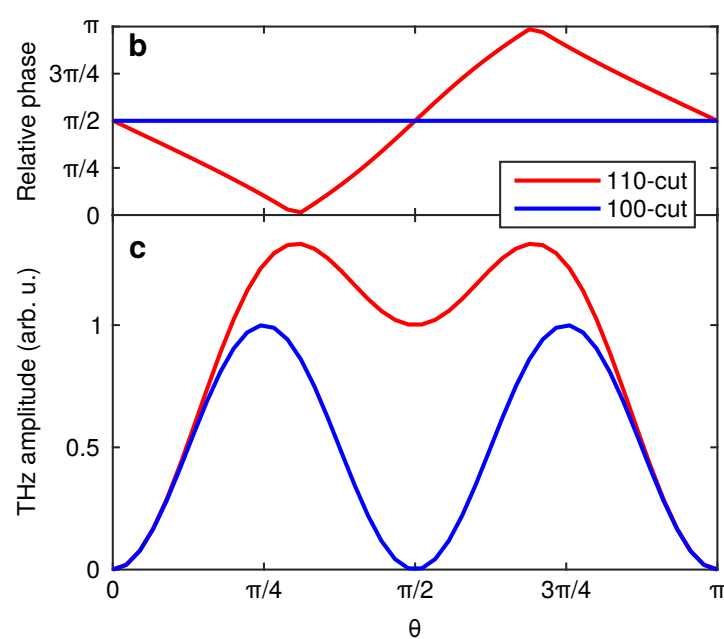

Figure 5.5.: (a) Emitted THz intensity as function of the crystal orientation. The radius corresponds to the azimuthal angle $\theta$ and the polar angle $\phi$ is the rotation angle, as defined in Eq. 5.12 The maximum T-ray intensity (red) are found for the polar angles $\phi \in\{\pi / 4,3 \pi / 4,5 \pi / 4,7 \pi / 4\}$. Therefore, (110)-cut crystals are chosen to generate THz. (b,c) shows a radial cut of the surface plot along $\phi=0$ and $\phi=\pi / 4$, which corresponds to normal incidence on a (100)-cut and a (110)-cut, respectively.

By cutting the surface plot accordingly, the azimuthal angular dependence $\theta$ is shown in Fig. 5.5. The (110) crystal orientation contains two global maxima located at $\theta \approx 54^{\circ}$ and at $\theta \approx 126^{\circ}$. In a (100)-cut crystal, the two local maxima are at $\theta \approx 45^{\circ}$ and $\theta \approx 135^{\circ}$. Note that in the maxima the generated $\mathrm{THz}$ pulse polarization is parallel to the incident light-pulse polarization. However, at the local minima $\left(\theta=90^{\circ}\right)$ the generated T-ray pulse is vertically polarized with respect to the incident light pulse polarization. The predicted behavior was experimentally confirmed by the author, see appendix A.2

\subsection{Phase-sensitive detection of coherent $\mathrm{THz}$ radiation}

Similar to the $\mathrm{THz}$ generation, there are lots of ways of how to detect $\mathrm{THz}$ radiation. High-sensitivity bolometers or pyroelectric detectors can be used to monitor the incident photon energy. In combination with narrow-band T-ray sources, they can be used to perform spectrally highly resolved T-ray experiments (e.g. in THz-FDS). In these setups, the frequency resolution is given by the bandwidth of the T-ray source. Since bolometers and pyroelectric detectors are only detecting the irradiated power, any phase information of the $\mathrm{THz}$ wave is lost. 
In a more complex $\mathrm{THz}$ time-domain spectroscopic approach, the information is recorded time-resolved. By temporally and spatially overlapping the $\mathrm{THz}$ pulse with a second NIR probe pulse and by tuning the relative delay time, the $\mathrm{THz}$ signal can be scanned in the time domain with a sub-picosecond resolution. As a result, a fast detector is realized by using a femtosecond probe beam. Since two photons can only interact via an exchange medium, a material with a strong coupling has to be found. Materials which show an electro-optical effect can be used as phase-sensitive detector crystals. Their refractive index $n$ is modified linearly by an externally applied electric field $E_{\text {ext }}$, making them birefringent while the field is applied [132, 154]:

$$
\Delta n=n_{0}^{3} r^{\mathrm{EO}} E_{\mathrm{ext}}
$$

where $n_{0}$ is the unmodified refractive index and $r^{\mathrm{EO}}$ is the electro-optical tensor. The electro-optical tensor is only allowed to be non-zero in non-centrosymmetric structures. The underlying effect is the Pockels effect [154, 155]. The THz-induced birefringence $\Delta n$ can be measured using a second transient light pulse. The linearly polarized probe beam will become elliptically polarized, with the ellipticity being proportional to the transient $\mathrm{THz}$ electric field.
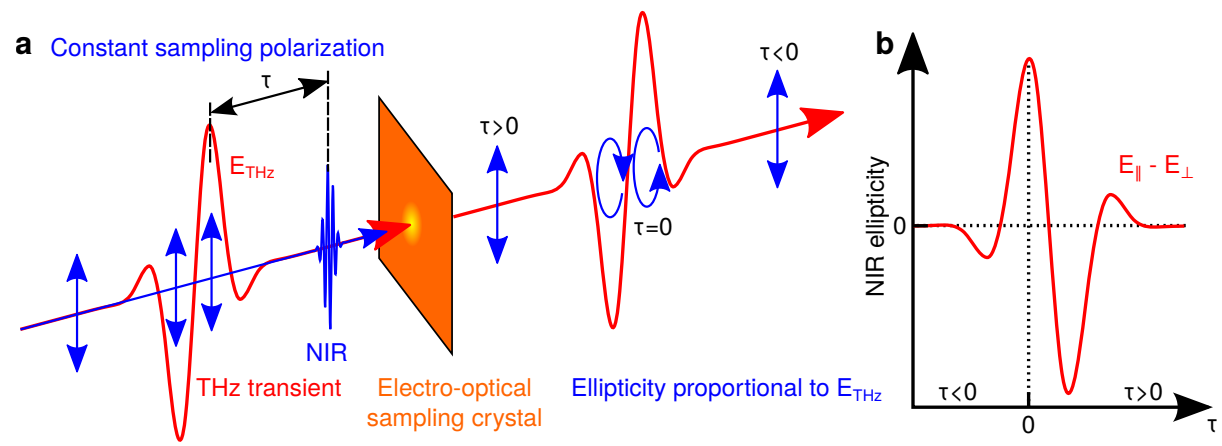

Figure 5.6.: Illustration of the Pockels effect. (a) The electric field of the T-ray transient (red) modifies the birefringence of the electro-optical sampling crystal (orange). Hence, depending on the temporal time delay $\tau$ between the $\mathrm{THz}$ transient (red) and the NIR probe beam (blue), the ellipticity of the probe beam is changing (indicated in blue after the electro-optical sampling crystal). (b) By monitoring the ellipticity of the NIR sampling beam as function of delay time $\tau$, the electric field of the T-ray transient is monitored phase-sensitively. The detection of the ellipticity is discussed in section 5.5.1.

For the duration of the $130 \mathrm{fs}$ sampling pulse, the THz-induced birefringence is assumed to be constant and the NIR sampling pulse becomes elliptically polarized according to Eq. 5.14. With a mechanical delay line, the electric field of the $\mathrm{THz}$ transient can be scanned by tuning the delay time $\tau$ of the sampling pulse. 
The detailed mathematical description of the electro-optical sampling process is presented in appendix B.1.

\subsubsection{Measuring the birefringence of the electro-optic sampling crystal}

In order to measure the electric field of the $\mathrm{THz}$ transient, the birefringence of the electro-optical sampling crystal has to be recorded [132. Birefringence is measured by sending a linearly polarized sampling beam through the electro-optical sampling crystal, as illustrated in Fig. 5.6.

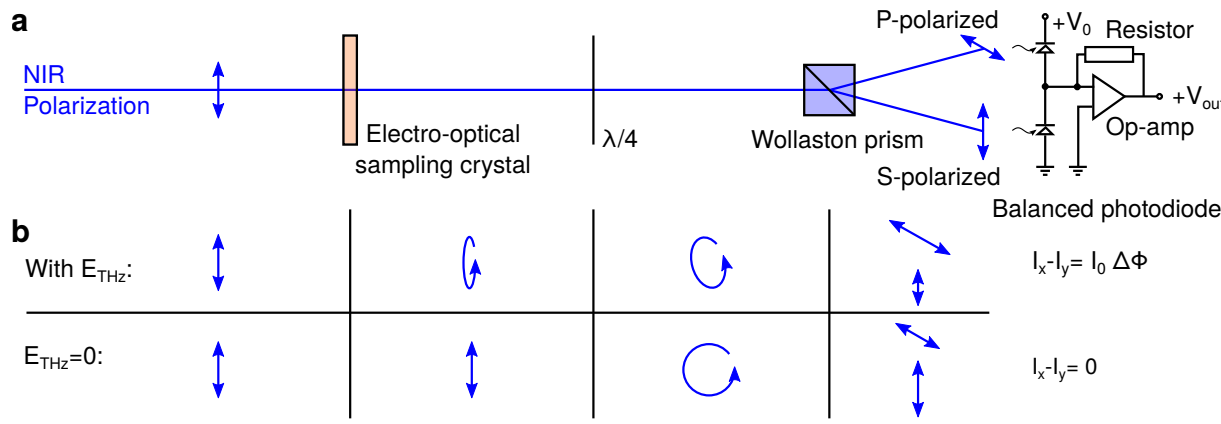

Figure 5.7.: Schematic diagram of a free-space electro-optical sampling as it was implemented by the author. (a) The NIR sampling pulse is sent linearly polarized onto the electro-optical sampling crystal, before passing a quarter-wave plate. The Wollaston prism spatially splits two perpendicular light polarizations. A balanced photodiode detects the difference between the two signals, which corresponds to the ellipticity of the NIR pulse after the electro-optical sampling crystal. (b) The NIR polarization is sketched in the case of a $\mathrm{THz}$ transient, as well as in absence of $\mathrm{THz}$ radiation. The signal measured by the balanced photodiode corresponds to ellipticity of the sampling pulse. $I_{\mathrm{x}}$ and $I_{\mathrm{y}}$ indicate the light intensity for s- and p-polarized light. By temporally shifting the sampling pulse, the electric field of the $\mathrm{THz}$ pulse can be tracked. Adopted with permission from 132 .

To measure the ellipticity of a NIR pulse, the beam is guided through a quarter-wave plate. It has to be set such that in absence of birefringence (in this case no $\mathrm{THz}$ electric field) the linearly polarized probe beam becomes circularly polarized. If a $\mathrm{THz}$ transient is inducing birefringence, the linearly polarized sampling beam will evolve into an elliptically polarized beam. A Wollaston prism is used to split the elliptically polarized light into two perpendicularly polarized light components. Using a balanced photodiode, the difference between the two optical branches is measured which is, according to Eq. 5.14 proportional to the electric field of the $\mathrm{THz}$ pulse. Fig. 5.7 illustrates the free-space electro-optical sampling. This detection scheme can be mathematically described using the so-called Jones calculus, see appendix B.2. 


\subsection{Carrier-envelope phases and transform limitations in wave packets}

A simple wave packet is characterized by its carrier frequency $\omega$ as well as the group velocity $\vec{v}_{\mathrm{g}}$ of its envelope. Furthermore, in a wave packet a carrier-envelope phase $\phi_{\mathrm{CE}}$ can be defined, as illustrated in Fig. 5.8. The carrier-envelope phase is only independent of time, if the following relation holds:

$$
\left|\vec{v}_{\mathrm{g}}\right|=\left|\vec{v}_{\mathrm{ph}}\right|=\frac{\omega}{k}
$$

where $\vec{v}_{\mathrm{ph}}$ is the phase velocity and $k$ the magnitude of the wave vector. A wave packet propagating along $x$ is described by the following equation:

$$
E(x, t)=G(x, t) \cdot e^{i(\omega t-k x+\phi)}=\frac{1}{\sqrt{2 \pi} \sigma} e^{-\frac{1}{2}\left(-\frac{x-v_{\mathrm{g}} t}{\sigma}\right)^{2}} \cdot e^{i(\omega t-k x+\phi)}
$$

where $G(x, t)$ is the envelope function. Here, a Gaussian envelope with a standard deviation $\sigma$ is assumed. Hence, under the assumption specified by Eq. 5.15. the wave packtet reads as:

$$
E(x, t)=\frac{1}{\sqrt{2 \pi} \sigma} e^{-\frac{1}{2}\left(-\frac{k x-\omega t}{\sigma k}\right)^{2}} \cdot e^{i\left(\omega t-k x+\phi_{\mathrm{CE}}\right)}
$$

where the phase $\phi$ becomes the time-independent carrier-envelope phase $\phi_{\mathrm{CE}}$. An illustration of different carrier-envelope phases $\phi_{\mathrm{CE}}$ is given in Fig. 5.8.

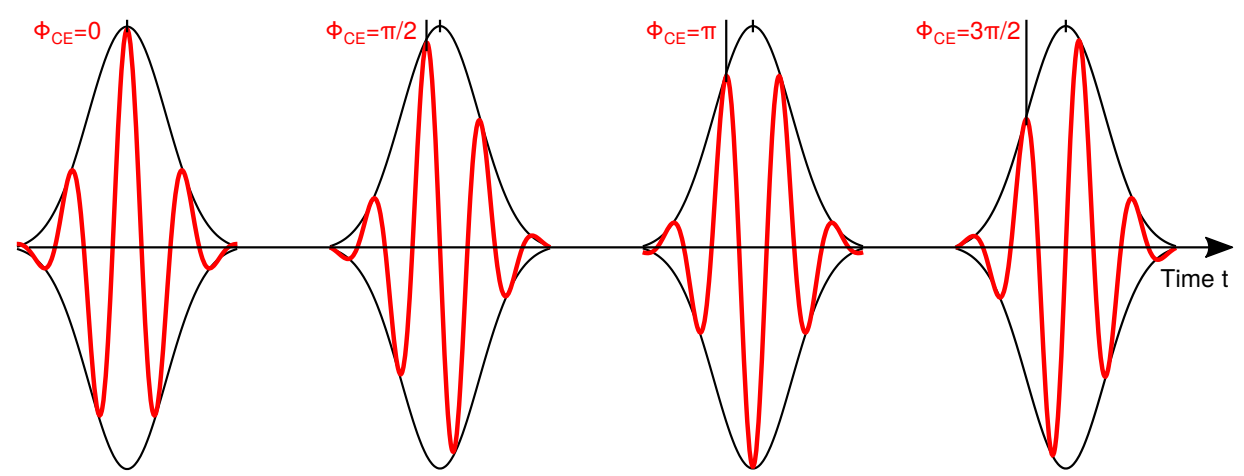

Figure 5.8.: Illustration of different carrier-envelope phases. Two signals with a relative carrier-envelope phase of $\pi$ are the same signals, but with reversed sign.

Note that the carrier-envelope phase does not have any effect on the spectrum of the pulse. However, the standard deviation $\sigma$ of the envelope has a major 
effect on the spectrum. In fact, a pulse with a standard deviation $\sigma_{\nu}$ in the spectrum has a lower limit of how small $\sigma$ can be, i.e. how strong a pulse can be compressed temporally. The restriction is purely due to the nature of the Fourier transform. The spectral width of any temporally confined laser pulse always fulfills the following inequality 152]:

$$
\Delta \nu \Delta t \geq K
$$

where $K$ depends on the envelope shape. $\Delta \nu$ and $\Delta t$ label the FWHM of the spectrum and the time envelope of the pulse $]^{9}$ For Gaussian envelopes, the numerical value of $\mathrm{K}$ is 0.441 . However, only so-called transform-limited pulses fulfill the equality in Eq. 5.18 and are therefore perfectly temporally compressed. This furthermore explains why ultrabroad light sources have to be used to achieve ultrashort light pulses. The aim of the author was to achieve transform-limited $\mathrm{THz}$ pulses in his setup, in order to have the shortest possible perturbation to investigate the intrinsic Kondo-lattice dynamics.

\subsection{Extraction of the non-equilibrium response}

In THz-TDS, the electric field is measured phase-sensitively in the time domain. Hence, a complex Fourier transform can be applied without any loss of information. As every $\mathrm{THz}$ transient has a characteristic spectrum, every sample spectrum has to be normalized to the original $\mathrm{THz}$ spectrum. Therefore, each acquired signal as to be compared to a reference measurement, in order to correct for the incident spectrum. In transmission geometry, the reference measurement is conducted simply by removing the sample. However, in reflection geometry, a good T-ray mirror (without any absorption) has to replace the sample. For this purpose, a platinum layer was used by the author to measure the incident T-ray spectrum, see Fig. 5.4. If the sample is investigated as function of an external parameter (e.g. temperature, magnetic field, etc.), the reference spectrum could also be collected at a reference value of the external parameter. By normalizing the measurement with the second, alternative method only the relative changes can be extracted.

In the time domain, the induced polarization $P(t)$ is described by a convolution between the linear response function $\chi(t)$ and the incident $\mathrm{THz}$ pulse $E^{\mathrm{THz}}(t)$ :

$$
P(t)=\int_{-\infty}^{\infty} \chi(\tau-t) E^{\mathrm{THz}}(t) \mathrm{d} \tau
$$

\footnotetext{
${ }^{9}$ For a Gaussian, the following equalities hold: $\Delta \nu=2 \sqrt{2 \ln 2} \sigma_{\nu}$ and $\Delta t=2 \sqrt{2 \ln 2} \sigma$
} 
The response function $\chi(t)$ can be retrieved by a deconvolution algorithm. However, in the frequency domain the response function is accessible by dividing the two spectra:

$$
\chi(\omega)=\frac{P(\omega)}{E^{\mathrm{THz}}(\omega)} .
$$

In other words, the complex linear response function $\chi(\omega)$ corresponds to the normalized complex spectrum. The complex dielectric function $\epsilon(\omega)$ as well as the complex conductivity $\sigma(\omega)$ are directly related, see section 5.8 . The major advantage of THz-TDS over THz-FDS is the direct access of the dielectric function, as the phase information is also recorded. Additionally, THz-TDS does not rely on any Kramers-Kronig modeling algorithms [156]. As a test, the evaluation scheme presented here can be used to measure the vibrational modes of molecules in the $\mathrm{THz}$ range. The measured eigenmodes of the $\mathrm{H}_{2} \mathrm{O}$ molecules are in excellent agreement with literature values. The experimental findings are presented in appendix C.1.

\subsection{Investigating metallic systems - reflection geometry}

As the aim of this thesis is to investigate heavy fermions and Kondo lattices using THz-TDS, the general electromagnetic response of metallic systems shall be briefly addressed. One of the most basic models describing the electromagnetic response of metals is the Drude-Lorentz model. Even though this model was derived with a conventional metal in mind, it seems to be also valid for heavy-fermion systems [122, 157]. Note that the Drude-Lorentz model describes the equilibrium response of a heavy fermion as it is measured using THz-FDS. However, the dynamical response of heavy fermions is expected to be different, since its band structure is time dependent due to the strong correlation. These aspects of the dynamical response of Kondo lattices is addressed in chapter 6 .

\subsubsection{Drude-Lorentz model for metals}

The Drude-Lorentz model is one of the most simple, yet intuitive models describing the frequency-dependent conductivity in a metal. Not only is it valid for a Fermi gas, but the same formalism can be applied to Fermi liquids. The key idea is that an external electromagnetic wave accelerates the mobile charge carriers. However, these charge carriers are scattering as described in chapter 2. The average time between two scattering processes is captured with the time constant $\tau$, which depending on the medium. It was shown that the relaxation rate $\tau$ is extremely slow in correlated electron systems [158. Nevertheless the Drude-Lorentz model 
seems to be still valid [122, 157] and the resulting equation of motion for the mobile electrons reads as follows [132]:

$$
\frac{\mathrm{d}}{\mathrm{d} t}\langle\vec{p}(t)\rangle=e\left(\vec{E}+\frac{\langle\vec{p}(t)\rangle \times \vec{B}}{m}\right)-\frac{\langle\vec{p}(t)\rangle}{\tau}
$$

where $\langle\vec{p}(t)\rangle$ is the average momentum vector of the mobile charge carriers, $e$ is the charge of the free carriers and $\vec{E}, \vec{B}$ describe the electric field and magnetic flux, respectively. Starting from this equation of motion, the complex frequency-dependent conductivity $\sigma(\omega)$ can be directly extracted [158]:

$$
\sigma(\omega)=\frac{\sigma_{0}}{1-i \omega \tau}
$$

where $\sigma_{0}$ is the DC conductivity. The real part of the complex conductivity $\sigma(\omega)$ describes the attenuation and the imaginary part the phase shift caused by the medium [158]. A relatively sharp drop of the real part of the complex conductivity emerges, as the frequency reaches the so-called Plasma frequency $\omega_{\mathrm{p}}$ [132]:

$$
\omega_{\mathrm{p}}^{2}:=\frac{N e^{2}}{\epsilon_{0} m^{*}}
$$

where $N$ is the number of free charge carriers, $\epsilon_{0}$ the vacuum permittivity and $m^{*}$ effective mass of the free charge carriers. Hence, for heavy fermions and their massively increased effective mass one would expect a very sharp Drude peak. However, this ultrasharp Drude peak has never been observed so far [125]. The dielectric permittivity $\epsilon(\omega)$ is directly linked to the conductivity [132]:

$$
\epsilon(\omega)=1+\frac{i \sigma(\omega)}{\omega \epsilon_{0}}
$$

as well as the complex linear response function $\chi$ :

$$
1+\chi=\frac{\epsilon(\omega)}{\epsilon_{0}}=\epsilon_{r}
$$

According to the author's knowledge, all optical experiments used to investigate Kondo lattices in the $\mathrm{THz}$ range were used to extract the optical conductivity $\sigma(\omega)$ and did not aim at gaining any temporal information [108, 111, 125, 159]. In contrast, the aim of this thesis is to use THz-TDS to investigate the heavy-fermion dynamics. The time resolution in THz-TDS allows us to observe the long, phase-coherent lifetime expected for $\mathrm{THz}$ excitations, see section 2.6 . 


\subsection{Experimental limitations in the time domain}

The evaluation discussed so far assumed analytical and therefore infinitely long time traces of the $\mathrm{THz}$ pulse. However, the experimentally acquired time traces have a finite interval length as well as a finite sampling rate, both of which impose restrictions with respect to the spectrum. This section is dedicated to address some issues resulting from the discretization of a continuous signal.

\subsubsection{Temporal sampling rate}

In an experiment, the time-domain signal has to be sampled with a finite sampling rate. Unfortunately, the step size $\Delta$ has some profound influence on the detected spectrum. In fact, the highest detectable frequency $\nu_{\max }$ strongly depends on $\Delta$ :

$$
\nu_{\max }=\frac{1}{2 \Delta}
$$

Eq. 5.26 is called Nyquist-Shannon theorem [160, 161. It states that the sampling rate has to be at least twice the maximum frequency in order to be deterministic. The Nyquist-Shannon sampling theorem can be illustrated as shown in Fig. 5.9.

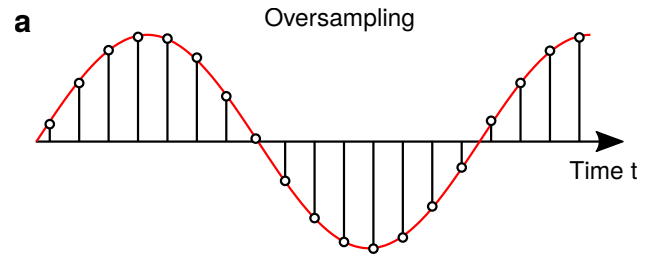

b

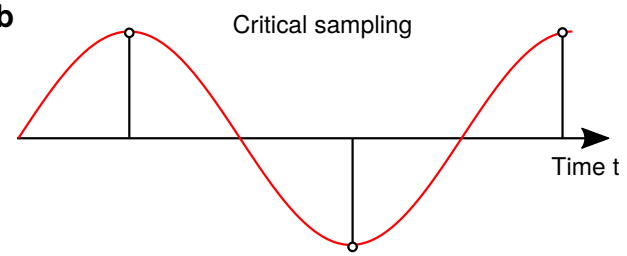

c

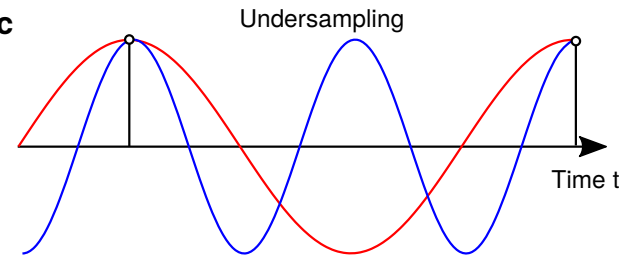

Figure 5.9: Illustration of over- and undersampling of a signal. (a) If the sampling rate is larger than specified by Eq. 5.26, the signal is oversampled. (b) If Eq. 5.26 is fulfilled, the signal is critically sampled. However, achieving critical sampling is difficult. (c) If the sampling frequency is less than what is specified by the Nyquist-Shannon theorem, the signal is no longer deterministic, as illustrated by the blue and red curve. The two curves cannot be distinguished unless the sampling rate is larger than $\nu_{\max }$, see Eq. 5.26 .

In the case of a discrete data set, the analytical Fourier transform has to be replaced by the discrete Fourier transform, as only $N$ sampling points $a_{j}$ are available: 


$$
\hat{a}_{k}:=\frac{1}{N} \sum_{j=1}^{\infty} e^{i \frac{2 \pi \cdot k j}{N}} a_{j}
$$

\subsubsection{Finite trace effects}

A typical time trace in THz-TDS is only a few ps long. Hence, some special treatment of the experimentally acquired data has to be done in order to avoid artifacts. The Fourier transform for finite time traces is defined as follows:

$$
f(\omega):=\frac{1}{T} \int_{T_{\text {start }}}^{T_{\text {end }}} f(t) e^{i \omega t} \mathrm{~d} t
$$

where $T$ is the time interval length of the measurement. However, by strictly applying Eq. 5.28, some artifacts are introduced. First, the resulting Fourier signal implies a periodic time trace with a periodicity $T$. These, reflectivity ${ }^{6}$ effects of the Fourier transform can be circumvented by artificially increasing the interval length. For a single pulse, as it is acquired in THz-TDS, the signal before the pulse is expected be zero, which can be either measured or artificially added. This procedure is called zero-padding [162]. However, the length of the time interval $T$ has a direct effect on the spectral resolution. If a frequency resolution $\Delta \nu$ is wanted, the time trace has to be at least of length $T_{\min }$ [162]:

$$
2 \cdot T_{\min }=\frac{1}{\Delta \nu}
$$

Increasing the length of the interval by measuring or artificially adding zeros increases the spectral resolution. However and obviously, the added interval does not contain any information and the higher spectral resolution is an artifact. In fact, this zero-padding technique has the same effect on the spectrum as an interpolation. An exemplification of this is presented in Fig. 5.10 


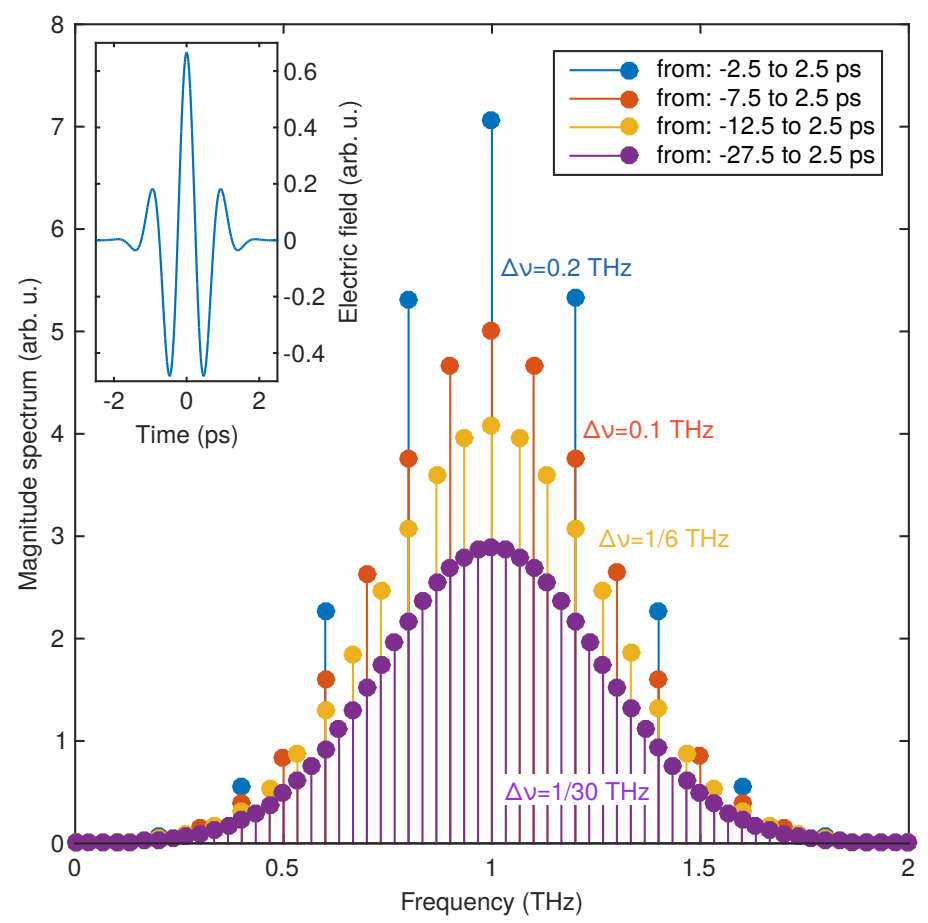

Figure 5.10.: Illustration of the effects of the interval length. The time trace is assumed to be $\pm 2.5 \mathrm{ps}$ (see inset). Note that the function of the inset is symmetric. Therefore, the frequency resolution of the spectrum is limited to $\Delta \nu=0.2 \mathrm{THz}$ (blue points). By zero-padding the signal, the overall array length and therefore the frequency resolution increases. By expanding the time signal from -7.5 to $2.5 \mathrm{ps}$, the frequency resolution is doubled: $\Delta \nu=0.1 \mathrm{THz}$ (red points). If the interval of the zero-padding is further expanded, the spectrum gets better resolved: $2 \mathrm{x}$ zero-padding equals $\Delta \nu=\frac{1}{6} \mathrm{THz}$ and $5 \mathrm{x}$ zero-padding corresponds to $\Delta \nu=\frac{1}{30} \mathrm{THz}$.

Alternatively, the time signal can also be multiplied with a function, which converges to zero towards the end of the measured time interval. Functions with these properties are called window functions [162]. Window functions are mainly used to analyze periodic signals which are recorded over a short time interval. Multiplying the signal with the window function guarantees that the signal becomes zero towards the boundaries of the interval. This is how abrupt jumps at the edges of the interval are avoided, which introduce noise to the spectrum [162]. For a single pulse signal as observed in THz-TDS, which is already zero at the interval edges, using a window function will have only minor effects. 


\subsection{Efficiency aspects and estimation of the electric field strengths}

As illustrated in section 5.4 the optical rectification process is a powerful tool to convert NIR laser pulses into T-ray pulses. For large-area ZnTe(110) emitters, as used by the author, the energy conversion efficiency is reported to be $1.5 \cdot 10^{-6}[163]$. However, the photon conversion efficiency is three orders of magnitude better: With each NIR pulse approximately $4 \cdot 10^{15}$ photons are converted into approximately $2 \cdot 10^{12}$ photons in the $\mathrm{THz}$ range.

The energy density flow in electromagnetic waves is described by the Poynting vector $\vec{S}$, which is defined as [164]:

$$
\vec{S}:=\vec{E} \times \vec{H}
$$

where $\vec{E}$ and $\vec{H}$ are the electric and magnetic field vectors 10 In the case of a pulsed light source, the pulse energy $\mathcal{E}_{\text {pulse }}$ can be measured by integrating the absolute value of the Poynting vector across the spatial cross section as well as time. Assuming a Gaussian profile in space and time, the pulse energy reads as follows:

$$
\begin{aligned}
\mathcal{E}_{\text {pulse }} & =\iiint \mathrm{d} x \mathrm{~d} y \mathrm{~d} t|\vec{S}(x, y, t)| \\
& =\iiint \mathrm{d} x \mathrm{~d} y \mathrm{~d} t \frac{1}{(2 \pi)^{\frac{3}{2}} \sigma_{\mathrm{x}} \sigma_{\mathrm{y}} \sigma_{\mathrm{t}}} \sqrt{\frac{\epsilon_{0}}{\mu_{0}}} e^{-\frac{x^{2}}{2 \sigma_{\mathrm{x}}^{2}}} e^{-\frac{y^{2}}{2 \sigma_{\mathrm{y}}^{2}}} e^{-\frac{t^{2}}{2 \sigma_{\mathrm{t}}^{2}}} E_{0}^{2} \cos ^{2}(\omega t) \\
E_{0}^{2} & =\sqrt{\frac{\mu_{0}}{\epsilon_{0}}} \frac{\mathcal{E}_{\text {pulse }}}{\iiint \mathrm{d} x \mathrm{~d} y \mathrm{~d} t \frac{1}{(2 \pi)^{\frac{3}{2}} \sigma_{\mathrm{x}} \sigma_{\mathrm{y}} \sigma_{\mathrm{t}}} e^{-\frac{x^{2}}{2 \sigma_{\mathrm{x}}^{2}}} e^{-\frac{y^{2}}{2 \sigma_{\mathrm{y}}^{2}}} e^{-\frac{t^{2}}{2 \sigma_{\mathrm{t}}^{2}} \cos ^{2}(\omega t)}} \\
= & \sqrt{\frac{\mu_{\text {pulse }}}{2}} \\
& \underbrace{\frac{\mathcal{E}_{\text {pulse }}}{2}}_{\epsilon_{0}},
\end{aligned}
$$

where $\sigma_{\mathrm{x}, \mathrm{y}, \mathrm{t}}$ are the spatial and temporal standard deviations of the Gaussian beam profile. Relevant and restrictive for many experiments is the maximal electric field strength $E_{\max }$. By decreasing the spatial cross section $\sigma_{\mathrm{x}, \mathrm{y}}, E_{\max }$ can be greatly enhanced, as shown in Eq. 5.32.

\footnotetext{
${ }^{10}$ In vacuum, the magnetic field $\vec{H}$ is proportional to the magnetic flux density $\vec{B}$, with a prefactor $1 / \mu_{0}$, where $\mu_{0}$ is the vacuum permeability. In vacuum, the additional relation holds: $|\vec{B}|=\frac{|\vec{E}|}{c}=\sqrt{\epsilon_{0} \mu_{0}}|\vec{E}|$, where $c$ corresponds to the speed of light and $\epsilon_{0}$ is the vacuum permittivity.
} 
Furthermore, by temporally compressing the pulse higher electric field strengths are reached:

$$
\begin{aligned}
E_{\max } & =\left|\vec{E}_{\max }\right|=\sqrt{|\vec{S}(0,0,0)|}=\sqrt{\frac{E_{0}^{2}}{(2 \pi)^{\frac{3}{2}} \sigma_{\mathrm{x}} \sigma_{\mathrm{y}} \sigma_{\mathrm{t}}}} \\
& =\sqrt{\frac{1}{(2 \pi)^{\frac{3}{2}} \sigma_{\mathrm{x}} \sigma_{\mathrm{y}} \sigma_{\mathrm{t}}} \sqrt{\frac{\mu_{0}}{\epsilon_{0}} \frac{E_{\mathrm{pulse}}}{2}}},
\end{aligned}
$$

In order not to damage the $\mathrm{THz}$ generation crystal, the NIR pump beam diameter is large on the $\mathrm{ZnTe}(110)$ generation crystal $(\sim 1 \mathrm{~cm})$. Hence, the generated T-ray beam profile at the large-area generation crystal corresponds to a spatial standard deviation of $\sigma_{\mathrm{x}, \mathrm{y}} \approx 4.2 \mathrm{~mm}$. However, the sample is typically located in a focus of the beam where $\sigma_{\mathrm{x}}^{\text {focus }}=\sigma_{\mathrm{y}}^{\text {focus }}$ is only approximately $0.42 \mathrm{~mm}$. Furthermore, the T-ray pulse is also a Gaussian in the time domain. Its FWHM is approximately $2 \mathrm{ps}$ (see Fig. 5.4), which corresponds to a standard deviation of $\sigma_{\mathrm{t}}=0.84 \mathrm{ps}$. Hence, a T-ray pulse with an energy of $1.5 \mathrm{~nJ}$ corresponds to the following maximum electric fields:

$$
\begin{array}{l|l}
\text { at the optical rectification crystal } & E_{\max } \approx 3.5 \cdot 10^{4} \mathrm{~V} / \mathrm{m}=0.35 \cdot \mathrm{kV} / \mathrm{cm} \\
\hline \text { at the focus } & E_{\max } \approx 3.5 \cdot 10^{5} \mathrm{~V} / \mathrm{m}=3.5 \mathrm{kV} / \mathrm{cm}
\end{array}
$$

The electric field strengths reached in THz-TDS are considerably larger than the electric field strengths generated by continuous-wave sources [156]. By further optimizing T-ray sources and the focusing properties of the T-rays, electric field strengths in the range of $\sim 100 \frac{\mathrm{MV}}{\mathrm{cm}}$ can be generated [165 169]. These electric fields are sufficiently large to induce nonlinear effects using $\mathrm{THz}$ radiation 170,172$]$. By tuning the carrier-envelope phase, asymmetric pulse shapes are achievable which can be used for THz-switching experiments 140. 


\section{Heavy-fermion dynamics in $\mathrm{CeCu}_{6-\mathrm{x}} \mathrm{Au}_{\mathrm{x}}$ and $\mathrm{YbRh}_{2} \mathrm{Si}_{2}$}

As explained and discussed in chapter 2, heavy-fermion systems show many physical phenomena which are characteristic for strong correlations. A detailed discussion of the various aspects involves complex many-body physics. Despite its complexity, a reasonably good understanding of heavy fermions was developed over the last decades [27, 38, 40, 42]. However, only a very little is known about the dynamical aspects of strongly correlated systems [173-175].

The major experimental limitation for investigating dynamics is set by the temporal resolution of the techniques used. In order to resolve processes on a picosecond time scale or below, optical techniques are the method of choice. Since the very beginning of the $\mathrm{THz}$ revolution, it was obvious that strongly correlated systems are the ideal candidates to be investigated using T-rays [37. However, most T-ray spectroscopy experiments use continuous-wave sources to investigate superconductors, ferromagnetics, multiferroics, Mott transitions as well as topological insulators and metamaterials [176 187]. Also heavy-fermion systems have been tackled using continuous-wave sources in THz-FDS setups [123, 124, 128, 188]. For THz-FDS tunable narrow-band T-ray sources (e.g. backward-wave oscillators) are used, as they can be gradually tuned over a long spectral range [188]. This type of measurements give access to the electromagnetic properties of heavy-fermion systems in equilibrium. However, in this chapter time-resolved terahertz spectroscopy (THz-TDS) is applied to explore the formation dynamics of strongly correlated Kondo lattices. Furthermore, this novel approach to strong correlation dynamics of Kondo-lattice systems also reveals new insights about the quasiparticle behavior in the quantum critical regime, see chapter 7 .

\subsection{Goals for time-resolved measurements of heavy fermions}

In order to investigate the dynamics of heavy fermions and the extraordinarily long lifetime of their heavy quasiparticles, only a very limited number of techniques are available. High-resolution photoemission experiments could be one possibility to access the picosecond time scale with sufficient energy resolution [36, 189, 190]. However, the fragile strongly correlated states are likely to be strongly modified 
while exposed to this ionizing radiation. The ionizing nature of photoemission experiments further forbids to investigate the intrinsic formation dynamics. In order to excite resonantly the heavy fermions, the photon energy should match the binding energy of the quasiparticles, which is in the meV range. Only like this, the strongly correlated state can be resonantly disintegrated. The experiments presented here revealed that the relaxation dynamics follows a remarkable behavior which turns out to be characteristic for heavy fermions, see section 6.3. This characteristic relaxation mechanism can be described by a rate-equation ansatz and was further confirmed by dynamical mean-field theory, see section 6.4

The low photon flux in $\mathrm{THz}$ experiments, together with the high reflectivity of metals, further guarantee for no heating effects. Furthermore, the experimental setup is limited to temperatures above $1.8 \mathrm{~K}$, where the effect of radiation heat is less crucial than for experiments in the $\mathrm{mK}$ range. In heavy fermions not only the dynamics of the quasiparticles, but also quantum critical aspects are illuminated by this new, temporally resolved approach. In terms of the effective mass of the quasiparticle, the $\mathrm{THz}$ radiation induces a momentary loss of the large effective electron mass $m^{*}$. From the upper perspective, an ideal Kondo-lattice system is $\mathrm{CeCu}_{6-\mathrm{x}} \mathrm{Au}_{\mathrm{x}}$, as its Kondo temperature is reported to be between $6 \mathrm{~K}$ and $7 \mathrm{~K}$, which implies slow quasiparticle dynamics [7, 14, 99, 102]. Because Kondo temperature characterizes the width of the coherent spectral weight, the time scale on which the strongly correlated ground state is formed scales with its inverse, see section 2.6. Remarkably, the delayed response does not show an instantaneous exponential decay, as one would expect for weakly correlated single-electron relaxation, see section 6.4.1. The heavy-fermion response is a compact pulse, which is well separated from the instantaneous reflex, caused by the light conduction electrons. This, dark time ${ }^{6}$ between the two pulses corresponds to the inverse effective Kondo temperature, as this is the time it takes to recreate the spectral weight at the Fermi surface 1 see section 6.2 .

\subsection{Heavy-fermion signature in $\mathrm{THz}$ time traces in $\mathrm{CeCu}_{6-\mathrm{x}} \mathrm{A} \mathbf{u}_{\mathrm{x}}$}

In order to investigate the dynamics of Kondo-lattice systems, the author designed and developed a THz-TDS which allows us to investigate metallic bulk samples (in reflection geometry) at low temperatures. The experimental setup is explained in great detail in chapter 5. The experiments were performed on samples with two chemical dopings: the magnetically ordered $\mathrm{CeCu}_{5.0} \mathrm{Au}_{1.0}$ reference compound as well as the quantum critical $\mathrm{CeCu}_{5.9} \mathrm{Au}_{0.1}$ sample. These two systems show distinctly different ground states, see section 4.1. The author demonstrates that the two systems show profoundly different $\mathrm{THz}$ time traces, see Fig. 6.1.

\footnotetext{
${ }^{1}$ The accumulation of spectral weight at the Fermi surface is the key signature of a strongly correlated Kondo state.
} 

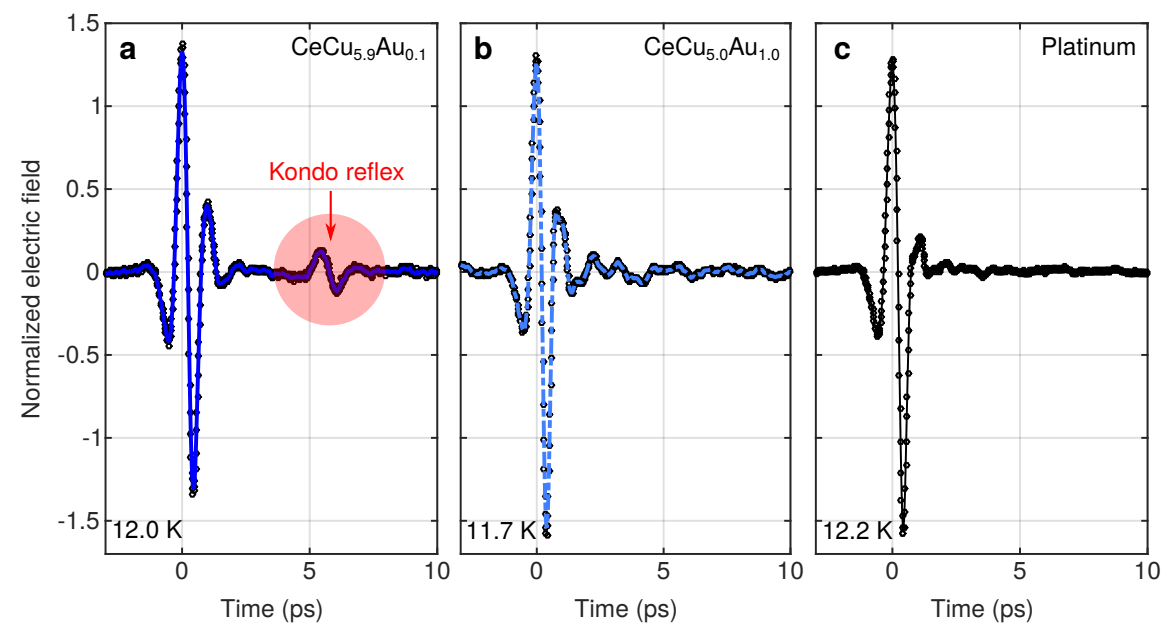

Figure 6.1.: Low-temperature, normalized time trace of various metallic systems. All curves have a normalized intensity between -3 and 9 ps. (a) The time trace of the reflected $\mathrm{THz}$ transient on a heavy fermion $\mathrm{CeCu}_{5.9} \mathrm{Au}_{0.1}$ sample. The data shows two pronounced features, a peak at $0 \mathrm{ps}$ and a signature after approximately $6 \mathrm{ps}$. The instantaneous pulse (at $0 \mathrm{ps}$ ) is caused by the light conduction electrons and is expected to appear in any metallic system. The temporally delayed, phase-coherent echo appears as the strongly correlated ground state is recreated (labeled in (a) as Kondo reflex). The $\mathrm{CeCu}_{5.0} \mathrm{Au}_{1.0}$ compound shows an antiferromagnetic ground state and is far from the heavy-fermion phase, see section 4.1.2. The time trace shows an instantaneous signal, which originates from the conduction electrons, but no time-delayed echo. The weak background is discussed in more detail in section 7.1.2. (c) Also the platinum mirror shows only an instantaneous reflex caused by the conduction electrons. Note that the Kondo reflex in (a) is not to be confused with trivial reflexes from the optical setup, see appendix D.

The quantum critical system $\mathrm{CeCu}_{5.9} \mathrm{Au}_{0.9}$ as well as two reference systems, the antiferromagnetic $\mathrm{CeCu}_{5.0} \mathrm{Au}_{1.0}$ and the platinum mirror, show a pronounced peak centered at $t=0$. This instantaneous response is expected for any kind of metallic system as it is the reflectivity caused by the light conduction electrons. Because the irradiated $\mathrm{THz}$ frequency is well below the plasma frequency $\omega_{\mathrm{p}}$ (see section 5.8) all three systems are highly reflective. Therefore, most of the irradiated $\mathrm{THz}$ transient is instantaneously reflected.

However, an additional and highly remarkable feature is observed in the $\mathrm{CeCu}_{5.9} \mathrm{Au}_{0.1}$ compound: At $t \approx 6 \mathrm{ps}$ a pronounced echo signal appears, well separated from the incident $\mathrm{THz}$ pulse. This eye-catching, delayed signature only appears for the heavy-fermion compound and is furthermore strongly temperature dependent. Hence, a direct link between the echo signature and the heavy-fermion property of $\mathrm{CeCu}_{5.9} \mathrm{Au}_{0.1}$ compound is self-evident. However, the emergence of a coherent echo signature after several picoseconds is remarkable, since the coherence 
time in metals is typically expected to be on a femtosecond time scale [191]. Note that this echo is not to be confused with trivial reflex pulses from the setup. These artifacts were all identified at different delay times, see appendix D.

\subsection{Phenomenological understanding of heavy-fermion signature}

In a first step, the time delay can be analyzed. From Fig. 6.2 the echo time $\tau_{\text {reflex }}$ can be estimated to be $5.8 \pm 0.1$ ps. This time scale can be converted to an energy scale, which for the delay time of the echo coincides remarkably well with the Kondo energy scale $T_{\mathrm{K}}^{*}$ of the heavy-fermion system:

$$
\frac{h}{k_{\mathrm{B}} \tau_{\text {reflex }}}=T \approx 8.4 \mathrm{~K} \approx T_{\mathrm{K}}^{*},
$$

where $h$ is the Planck constant and $k_{\mathrm{B}}$ the Boltzmann constant. A delay time scaling with the inverse Kondo temperature means that for heavy-fermion systems with sufficiently low $T_{\mathrm{K}}^{*}$, the Kondo echo is well separated from the conduction electron response. Hence, a well-separated echo signature enables a virtually background-free analysis of the Kondo signature.

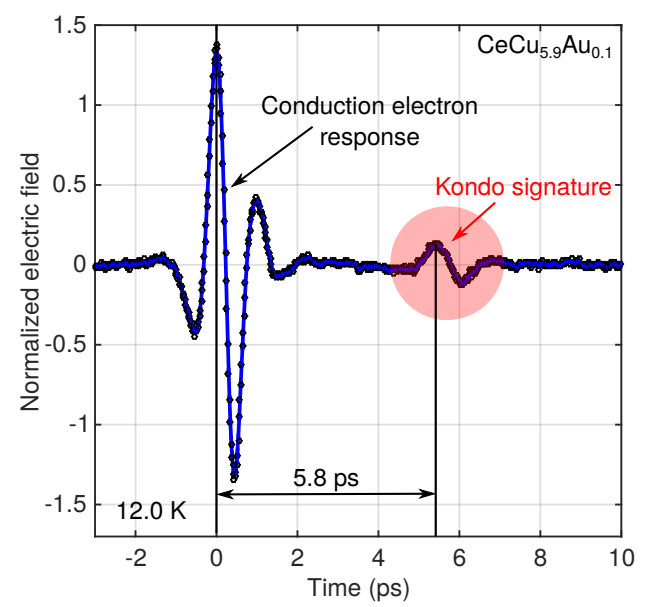

Figure 6.2: Low-temperature, normalized $\mathrm{THz}$ electric field reflected from the surface of a quantum critical $\mathrm{CeCu}_{5.9} \mathrm{Au}_{0.1}$ crystal. The intensity of the $\mathrm{THz}$ pulse is normalized to 1 (interval: -3 and $9 \mathrm{ps}$ ). The instantaneous response is due to the conduction electrons. The temporally delayed echo signature is emitted as the Kondo-lattice ground state re-emerges. The delay time of the Kondo signature is $5.8 \pm 0.1$ ps. This time scale corresponds to the inverse effective Kondo energy scale $T_{\mathrm{K}}^{*}$ of the system, see Eq. 6.1.

An upper limit for the delay time for the echo signature might be given by dephasing processes in the system, caused by scattering processes. However, this limitation is strongly depending on the system and making accurate predictions about scattering processes is very involving. So far, a first plausibility check for verifying the relation between the echo signal and the heavy-fermion state is made. However, 
this does not explain why an echo signal emerges in the first place. However, this can be understood by a simple rate-equation ansatz. Furthermore, the effects of an irradiated $\mathrm{THz}$ pulse on the band structure of a heavy-fermion system can be calculated using dynamical mean-field theory, see section 6.4.2. Hence, the ultimate step would be to calculate the full time-dependent band structure, which is an elaborate task, but not required for a general understanding of strong correlation dynamics.

\subsection{Characteristic strong correlation dynamics}

\subsubsection{Phenomenological rate equation ${ }^{2}$}

Rate equations are generally used tools to describe macroscopic relaxation relaxation behavior. They have the advantage of phenomenologically describing these relaxation processes without requiring a microscopic description of the underlying mechanisms. A rate equation can also be formulated for the relaxation dynamics of weakly and strongly correlated systems. From the discussion in chapter 2 it is known that in Kondo-lattice systems the Kondo spectral weight hybridizes with the conduction band, forming the shallow heavy-fermion band at the Fermi energy. This avoided crossing at $E_{\mathrm{F}}$ pinpoints the strong correlation effect in a Kondo-lattice system. If the Kondo state (i.e. the strong correlation) is disintegrated by a disruption at $t=0$, the band structure instantaneously changes. Since the strong correlation is ripped apart, the Kondo spectral weight at the Fermi surface disappears. In fact it is shifted back down to the magnetic core level. In other words, the effective mass of the electron gets instantaneously diminished by the incident $\mathrm{THz}$ transient.

Because the pulse energy of the generated $\mathrm{THz}$ radiation is only in the order of a few nJ (see section 5.10 and because a high percentage is instantaneously reflected by the conduction electrons (approximately 99\%, see section 6.5.2), the $\mathrm{THz}$ perturbation can be assumed to be of non-thermal nature. Hence, the intrinsic reformation time scale of the strong correlation ground state is observed. The time scale of this complex process is given by the inverse effective Kondo temperature, see section 2.6.

Weak correlation. The pinpoint of weakly correlated systems is the general robustness of the band structure against any perturbations. For example in a semiconductor the electronic structure remains unchanged if electrons are photoexcited. A simplified semiconductor band structure has only two energy levels in the vicinity of the Fermi surface, where the lower energy level $E_{\mathrm{v}}$ (valence band) is fully occupied with $N_{0}$ electrons and the upper band $E_{\text {c }}$ (conduction

${ }^{2}$ The concepts presented in this section were mainly developed in collaboration with J. Kroha and his research team from the Physikalisches Institut and Bethe Center for Theoretical Physics of the University of Bonn. 
band) is completely empty in the case of $T=0 \mathrm{~K}$. If electrons are photoexcited at $t=0, N$ electrons are transferred into the conduction band under the absorption of $N$ photons. The number of photoexcited electrons is time dependent, as the system will relax back into its ground state for $t>0$. This time dependence of the occupation number is captured by the following rate equation:

$$
\frac{\mathrm{d} N(t)}{\mathrm{d} t}=-\Gamma_{\mathrm{c} \rightarrow \mathrm{v}} N(t)
$$

where $N(t)$ is the time-dependent number of photoexcited electrons and $\Gamma_{\mathrm{c} \rightarrow \mathrm{v}}$ labels the transition rate. Fermi's golden rule allows us to calculate the transition rate $\Gamma_{\mathrm{c} \rightarrow \mathrm{v}}$, which defines the inverse lifetime of the excited state:

$$
\Gamma_{\mathrm{c} \rightarrow \mathrm{v}}=\frac{2 \pi}{\hbar} \rho\left(E_{\mathrm{v}}\right)\left|\left\langle\phi_{\mathrm{c}}\left|\mathcal{H}^{\prime}\right| \phi_{\mathrm{v}}\right\rangle\right|^{2}=: \frac{1}{\tau}=\text { const. },
$$

where $\rho\left(E_{\mathrm{v}}\right)$ is the DoS of the valence band and $\left|\left\langle\phi_{\mathrm{c}}\left|\mathcal{H}^{\prime}\right| \phi_{\mathrm{v}}\right\rangle\right|$ depicts the transition matrix element between the two bands. Hence, weakly correlated systems have a time-independent transition rate $\Gamma_{\mathrm{c} \rightarrow \mathrm{v}}$, leading to the linear differential rate equation:

$$
\frac{\mathrm{d} N(t)}{\mathrm{d} t}=-\frac{1}{\tau} N(t)
$$

Therefore, the weakly correlated system will relax exponentially on the time scale given by $\tau$ :

$$
N(t)=N_{0} e^{-\frac{t}{\tau}}
$$

While the system relaxes back into the equilibrium, the previously absorbed energy has to be released. Assuming that the energy is released under the re-emission of photons, the envelope of the radiation $\bar{E}(t)$ is proportional to the time derivative of $N(t)$. Since $N(t)$ is an exponential function, the envelope of the re-emitted radiation is also exponential. 

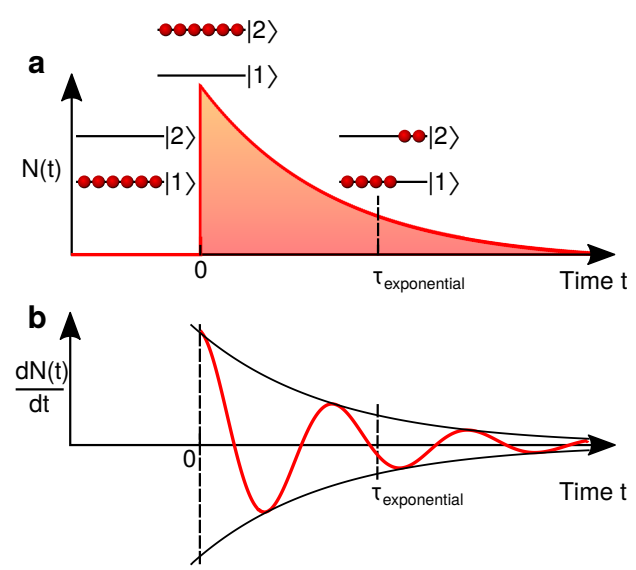

Figure 6.3: (a) Illustration of the time evolution of the number of photoexcited electrons $N(t)$ in a weakly correlated system. For $t<0$, the system is in equilibrium and all electrons are in the ground state. At $t=0$, the electrons get photoexcited and occupy the excited state, while the lower energy level remains empty. For $t>0$, the number of photoexcited electrons $N(t)$ reduces exponentially as the electrons repopulate the lower energy level. (b) The electric field envelope is given by the temporal derivative of $N(t)$. Hence, the amplitude of the emitted electric field is also exponential. For more details, see text.

Strong correlation. The relaxation of a heavy-fermion system can be described using an analogous rate-equation ansatz:

$$
\frac{\mathrm{d} N(t)}{\mathrm{d} t}=-\Gamma_{\mathrm{c} \rightarrow \mathrm{hf}} N(t)
$$

However in contrast, the DoS of the final state is now no longer constant. The reason has to be found in the strong correlation itself, which states that the heavy band only exists if the strong correlation is present. $N_{0}$ strongly correlated electrons are assumed to be bound in the heavy-fermion band. At $t=0, N$ of these electrons are excited and therefore breaking up the strongly correlated ground state. This loss of strong correlation greatly changes the DoS of the heavy-fermion ground state. As a result, the transition rate is now time dependent:

$$
\Gamma_{\mathrm{c} \rightarrow \mathrm{hf}}=\frac{2 \pi}{\hbar} \rho\left(E_{\mathrm{hf}}\right)\left|\left\langle\phi_{c}\left|\mathcal{H}^{\prime}\right| \phi_{\mathrm{hf}}\right\rangle\right|^{2}=\frac{4 \pi}{\tau_{\mathrm{K}}^{*}} \cdot \frac{N_{0}-N(t)}{N_{0}}, \quad \tau_{\mathrm{K}}^{*}=\frac{h}{k_{\mathrm{B}} T_{K}^{*}} .
$$

With a time-dependent transition rate, the rate equation turns into a quadratic first-order differential equation:

$$
\frac{\mathrm{d} n}{\mathrm{~d} t}=-\frac{4 \pi}{\tau_{\mathrm{K}}^{*}}(1-n) \cdot n
$$


where $n(t):=N(t) / N_{0}$ is the normalized number of photoexcited electrons. Quadratic first-order differential equations can be analytically solved, see appendix E. The solution reads as follows:

$$
n(t)=-\frac{1}{2} \tanh \left(\frac{2 \pi t}{\tau_{\mathrm{K}}^{*}}-1\right)+\frac{1}{2} .
$$

Assuming a re-emission of the photons, the envelope of the system response $\bar{E}(t)$ is the time derivative of the occupation number $N(t)$. Hence, the expected strong correlation response function looks as follows:

$$
\bar{E}(t)=\frac{1}{\cosh ^{2}\left(2 \pi\left(\frac{t}{\tau_{\mathrm{K}}^{*}}-1\right)\right)} .
$$

This solution of the rate equation is illustrated by Fig. 6.4.

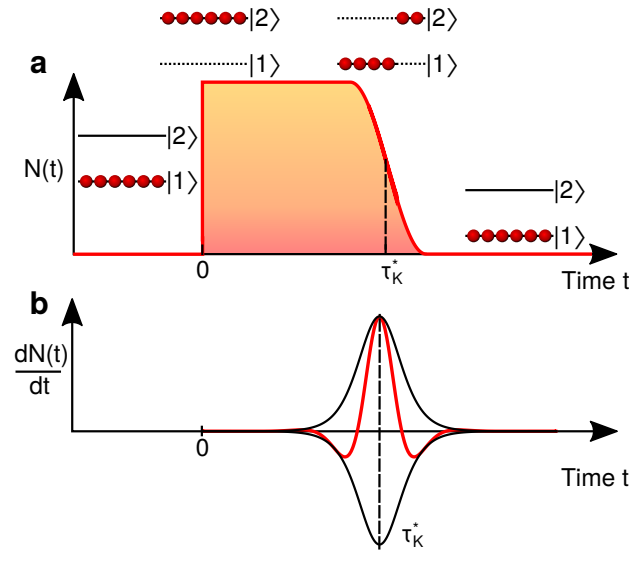

Figure 6.4: (a) Illustration of the time evolution of the number of photoexcited electrons $N(t)$ in a strongly correlated system. For $t<0$, the system is in its ground state. At $t=0$, electrons get photoexcited and populate the excited state. Due to the strong correlation, the lower energy state is destroyed as the electrons get excited (indicated by dotted line). Hence, the photoexcited electrons are trapped in the energetically unfavorable state and $N(t)$ remains constant. Only after a characteristic time $\tau_{\mathrm{K}}^{*}$, the strong correlation state re-establishes and allows a relaxation of the electrons into their ground state. $N(t)$ reduces accordingly. (b) The electric field envelope is described by the temporal derivative of the number of photoexcited electrons. This resembles an ,avalanche-like' behavior. For more details, see text. 
Summary. The strong correlation response looks distinctively different from the weak correlation dynamics. In a striking exemplification, this can be understood as follows:

At the disruption $t=0$, the heavy electrons are excited from the heavy-fermion band into the light part of the conduction band. This photoexcitation process breaks the Kondo singlets and correspondingly erases the the Kondo spectral weight (associated with the heavy band) at the Fermi surface. Therefore, the photoexcited electrons are trapped in the conduction band, as the time $\tau_{\mathrm{K}}^{*}=h / k_{\mathrm{B}} T_{\mathrm{K}}^{*}$ is required to recreate the spectral weight. Only after the recreation of the heavy band, the photoexcited electrons relax under the re-emission of the previously absorbed $\mathrm{THz}$ pulse. This relaxation mechanism boosts the strong correlation, which causes this ,avalanche-like' response shown in Fig. 6.4 The coherence is preserved since the phase space for the scattering of these photoexcited electrons is tiny, especially if compared with optical excitation experiments. The reason is to be found in the photon energy of the T-rays, which is three orders of magnitude less and therefore the coherence time is increased by several orders of magnitude.

Note that the $\mathrm{THz}$ echo pulse provides a direct measure of the spectral weight and of the effective Kondo temperature. The time scale $\tau_{\mathrm{K}}^{*}$ is governed by the inverse Kondo temperature and the Kondo spectral weight describes the probability for quasiparticle formation. With THz-TDS, both quantities can be observed within a single experiment. This description of strongly correlated relaxation dynamics is in excellent agreement with the observed relaxation dynamics shown in Figs. 6.1 and 6.2 .

\subsubsection{Dynamical mean-field theory}

Solving a Hamiltonian describing a strongly correlated electron system is highly demanding, even simplified versions of them (see section 2.5. Furthermore, it is difficult to tell whether a simplified Hamiltonian still captures the essence of the strong correlation. These circumstances asked for the development of new approaches to treat these types of Hamiltonians. In the late 1980s, pioneering work was done for the developments of the so-called dynamical mean-field theory (DMFT) 192.

The essential idea of DMFT is to describe a strongly correlated ground state (e.g. a Kondo lattice) using a single-site quantum impurity Hamiltonian. To capture the interaction amongst the individual sites, the single-site impurity is embedded self-consistently into an effective medium. Hence, the self-consistency takes care of the coherence phenomena as well as the periodicity of the structure. Such an impurity problem description has the advantage that many mathematical tools are available. From this perspective, DMFT can be also called a local impurity self-consistent approximation. In DMFT calculations the spatial fluctuations are frozen (single impurity description) while the local fluctuation of the different quantum states are fully taken into account. This allowance of local fluctuations is the major difference between mean-field theory in the 
sense of Hartree-Fock, where all fluctuations are frozen. Hence, this locally allowed ,dynamics' makes the local impurity self-consistency approximation a ,dynamical' mean-field theory [193]. Over the past twenty years, DMFT has proven to be a powerful approximation method allowing for a precise description of strongly correlated electron systems. Also Kondo-lattice systems are extensively calculated and analyzed using DMFT [194, 195]. Furthermore, various reviews about DMFT have been published [193, 196].

Our collaborator ${ }^{3}$ are applying DMFT to predict physical phenomena in strongly correlated systems. In particular, they calculated the band structures describing the time-resolved experiment presented in this thesis, where a Kondo-lattice system is perturbed at $t=0$. They calculated the Kondo-lattice band structure for two scenarios: in the equilibrium case at $t<0$ and for $t \rightarrow \infty$, as well as at the very instance of perturbation by the non-ionizing light pulse $(t=0)$ [11]. The resulting band structures are shown in Fig. 6.5.

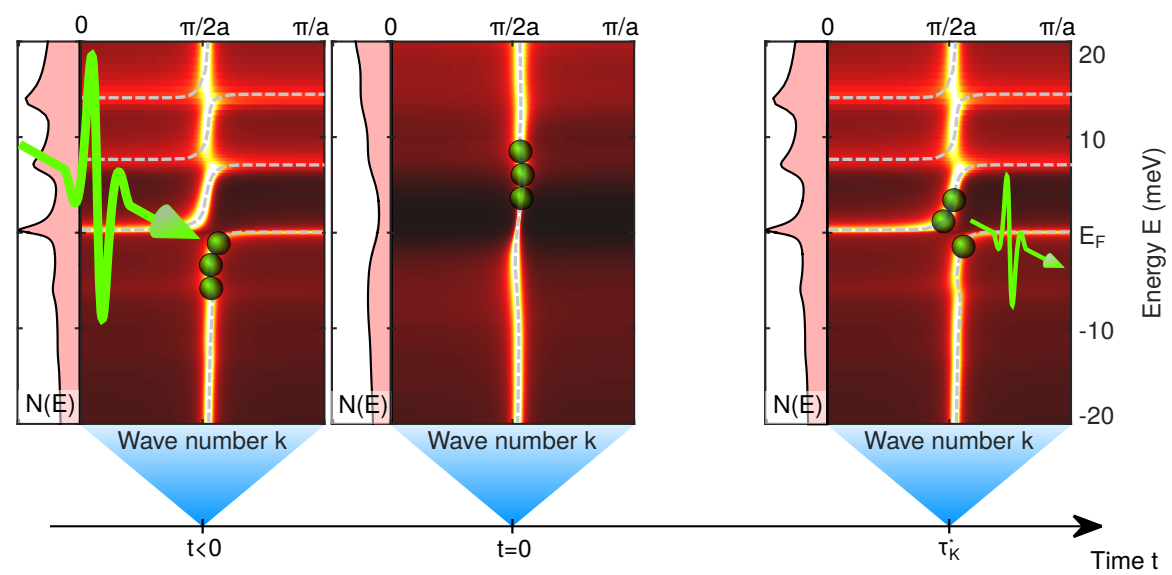

Figure 6.5.: DMFT calculations show the time-resolved solution of the Anderson Hamiltonian for a perturbed Kondo-lattice system at $t=0$. For $t<0$, the Kondo band as well as its satellites have fully emerged. $N(E)$ displays the $k$-integrated DoS. At the actual disruption time $t=0$, the band structure is perturbed. In fact, the perturbation , burns ${ }^{*}$ a hole in the band structure and the corresponding DoS. Also the CF satellites are destroyed as the DoS shows some dents. The author refers to this feature as the Kondo hole. For the simulation, the literature values of the quantum critical compound $\mathrm{CeCu}_{5.9} \mathrm{Au}_{0.1}$ were used. The perturbation is assumed to have a Gaussian spectrum centered at $1 \mathrm{THz}$. For more details, see text.

The DMFT calculations were performed using the multi-orbital Anderson-lattice model, by employing a multi-orbital non-crossing approximation impurity solver [11]. Calculating the full time evolution of the band structure is very

\footnotetext{
${ }^{3}$ The DMFT calculations were performed by the research group headed by J. Kroha from the Physikalisches Institut and Bethe Center for Theoretical Physics of the University of Bonn.
} 
demanding and thus subject of current investigations. However, the already performed DMFT band structure calculations confirm the THz-induced destruction of the strong correlation. In fact, they even show a drastic reduction of the DoS at the Fermi surface at $t=0$. The actual reduction of the 4 f spectral weight at $E_{\mathrm{F}}$ is best described as Kondo hole, which is induced by the $\mathrm{THz}$ radiation. The lifetime of this Kondo hole is inversely proportional to the effective Kondo temperature $T_{\mathrm{K}}^{*}$, as argued in section 6.4 .1

The $\mathrm{THz}$ perturbation in the DMFT calculations is assumed to have a Gaussian energy profile, centered at $4.1 \mathrm{meV}(1 \mathrm{THz})$ and a corresponding FWHM of $4.1 \mathrm{meV}$. The reduction of the CF satellites in the band structure is a particularly interesting feature revealed by the DMFT calculation, since the perturbation energy is well below the actual CF satellite energies. With the destruction of the Kondo singlets, the entire strong correlation collapses which also includes all the CF satellites surrounding the Kondo resonance. The DMFT calculations show that the avoided crossings, also for the CF satellites, disappear.

The emergence of the strongly correlated state as well as the time-dependent $4 \mathrm{f}$ spectral weight are the key to this complex dynamics. DMFT calculations are in excellent agreement with the author's experimental observation, as well as with the previously derived phenomenological rate-equation ansatz. Furthermore, the author observed experimental signatures which most likely originate from this very first $\mathrm{CF}$ satellite peak, see section 6.6.3. However, the intrinsic energy resolution in THz-TDS is a limiting factor for the observation of the CF satellites.

\subsection{Conformity of experiment and rate-equation ansatz}

According to the rate-equation approach showed in section 6.4.1, the delay time $\tau_{\mathrm{K}}^{*}$ is directly linked to the inverse effective Kondo temperature $T_{\mathrm{K}}^{*}$ :

$$
\tau_{\mathrm{K}}^{*}=\frac{h}{k_{\mathrm{B}} T_{\mathrm{K}}^{*}} .
$$

The time delay of the reflex pulse in $\mathrm{CeCu}_{5.9} \mathrm{Au}_{0.1}$ is $5.8 \mathrm{ps}$ (peak-to-peak, see Fig. 6.2, which corresponds to an effective Kondo temperature of $8.3 \mathrm{~K}$. As the reflectivity of the metallic $\mathrm{CeCu}_{6-\mathrm{x}} \mathrm{Au}_{\mathrm{x}}$ samples remain very close to 1 throughout the experiment, the normalized time-resolved signals are analyzed, i.e. the integrated pulse intensity on an interval between -3 and 9 ps is normalized [197]. The intensity of the echo pulse contains information about the Kondo spectral weight accumulated at the Fermi surface, see section 7.1. The proposed envelope function has only one single parameter $\tau_{\mathrm{K}}^{*}$, see Eq. 6.10. Note further that the resulting envelope is in excellent agreement with the experimental observations, see Fig. 6.6. 

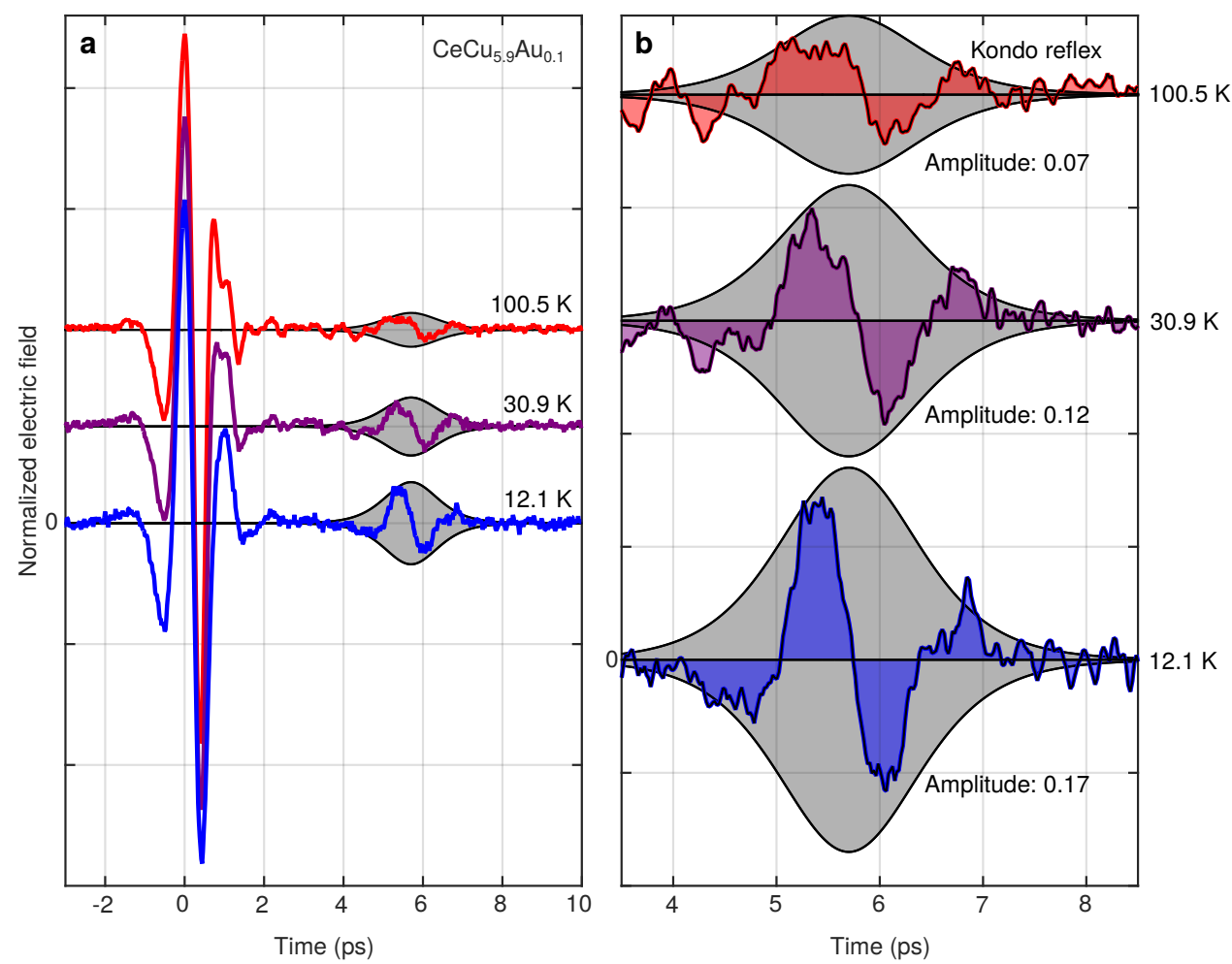

Figure 6.6.: (a) The temperature-dependent and normalized $\mathrm{THz}$ electric field reflected from a $\mathrm{CeCu}_{5.9} \mathrm{Au}_{0.1}$, with the envelope function (gray) derived in section 6.4.1 and an effective Kondo temperature of $8.3 \mathrm{~K}$. The intensity of all time traces are normalized on an interval between -3 and 9 ps. With decreasing temperature, only the amplitude of the $1 / \cosh ^{2}$ envelope function has to be rescaled to match the Kondo resonance signal. (b) The zoomed image of the Kondo reflex reveals how the amplitude changes with decreasing temperature.

This proposed interpretation of the reflex pulse allows us to monitor simultaneously the Kondo temperature as well as the accumulated Kondo spectral weight at the Fermi surface. Since the heavy-fermion response is fully determined by the effective Kondo temperature (also the width of the envelope scales with $T_{\mathrm{K}}^{*}$ ), the delayed pulse is spectrally shifted towards lower frequencies. This spectral shift is expected to grow linearly with the Kondo delay time $\tau_{\mathrm{K}}^{*}$, see Fig. 6.7. 


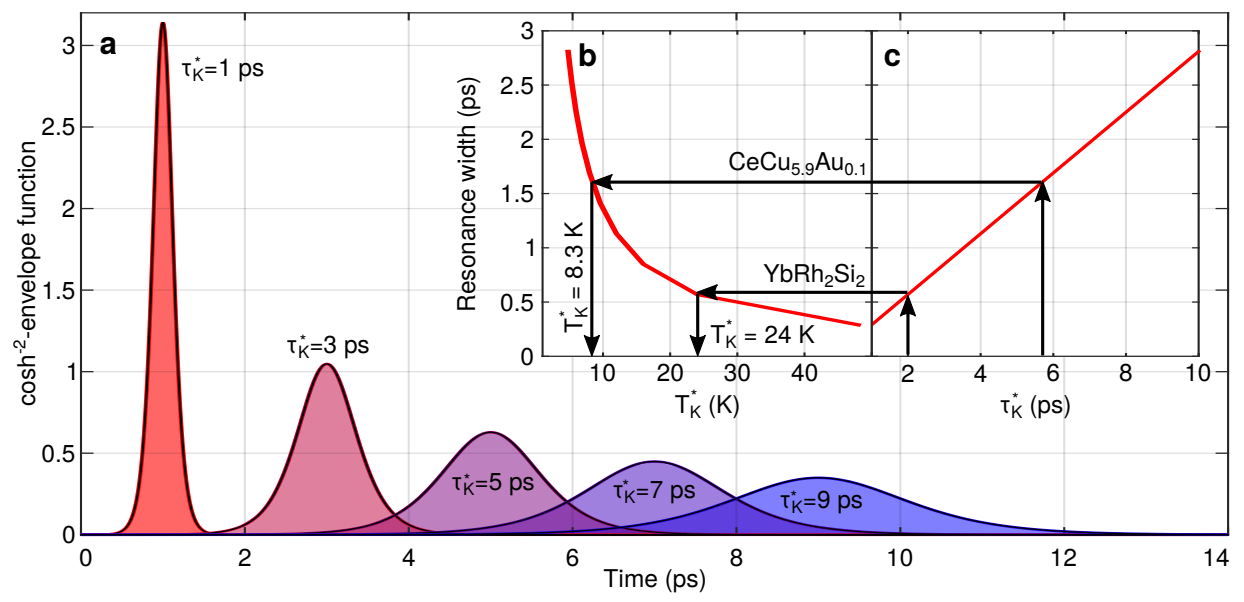

Figure 6.7.: (a) Simulated $\cosh ^{-2}$ envelope behavior as function of the Kondo delay time $\tau_{\mathrm{K}}^{*}$, as depicted by Eq. 6.10. Furthermore, the resonance width (FWHM of the envelope function) scales with $\tau_{\mathrm{K}}^{*}$, as shown in inset (c). (b) The Kondo delay time $\tau_{\mathrm{K}}^{*}$, but also the resonance width, are directly connected to the Kondo temperature. By measuring one of the two quantities, the Kondo temperature can be extracted, as indicated by the arrows for two investigated Kondo systems.

Fig. 6.7 implies that for large Kondo delay times $\left(\tau_{\mathrm{K}}^{*} \geq 10 \mathrm{ps}\right)$ the echo pulse will be strongly dispersed. This temporal spreading of the heavy-fermion response has to be taken into account, when selecting an appropriate system for the experiment.

\subsubsection{Proof of phase coherence}

Phase-coherent echo signals, as observed for heavy fermions, have characteristic properties. For instance, if the incident pulse shape is modified, the echo will be modified accordingly. The author demonstrates this by tuning the $\mathrm{THz}$ transient, which will affect the shape of the echo as well, see Fig. 6.8

Furthermore, by rotating the $\mathrm{THz}$ generation crystal by $\pi$, the $\mathrm{THz}$ transient can be inverted, see section 5.4. As expected for a phase-coherent process, the echo signature is also inverted, see Fig. 6.9. 


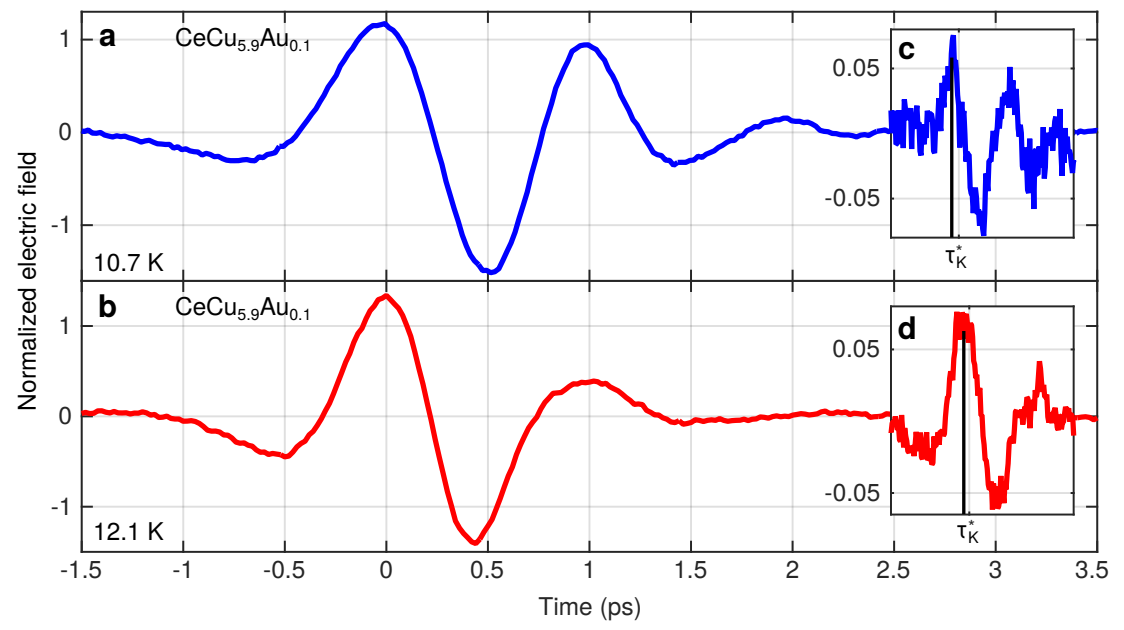

Figure 6.8.: Observation of the Kondo echo by tuning the THz transient. (a,b) Two different $\mathrm{THz}$ transients are reflected of a $\mathrm{CeCu}_{5.9} \mathrm{Au}_{0.1}$ surface. At sufficiently low temperatures, the Kondo signal emerges. (c,d) The insets show the Kondo echo for the corresponding $\mathrm{THz}$ transients. The shape of the echo pulse strongly resembles the incident $\mathrm{THz}$ transient. This proves the coherence-conserving property of the Kondo dynamics. The THz intensity in both cases was normalized on the interval from -3 to 8 ps. The experiment was conducted between 11 and $12 \mathrm{~K}$.

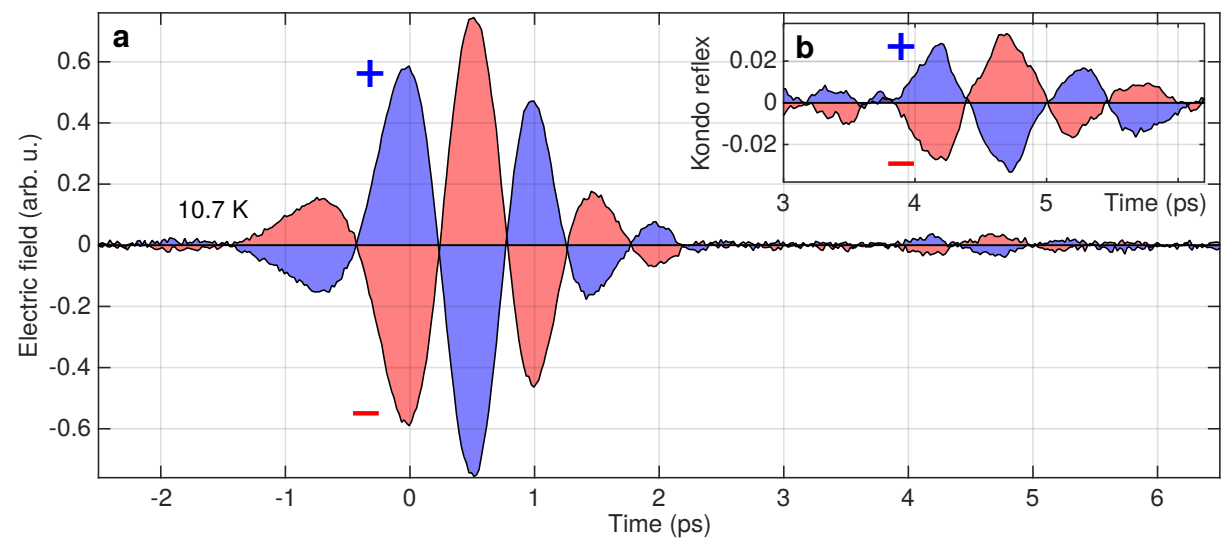

Figure 6.9.: Observation of the Kondo echo for an inverted $\mathrm{THz}$ transient. (a) Flipping the $\mathrm{THz}$ transient (indicated by,$+^{6}$ and,$-^{-}$) causes a flip in the echo signal. Inset (b) shows the Kondo echo, which is inverted accordingly. This measurement was conducted on a $\mathrm{CeCu}_{5.9} \mathrm{Au}_{0.1}$ sample at a temperature of $10.7 \mathrm{~K}$. 


\subsubsection{Kondo-echo intensity versus $\mathrm{THz}$ pump intensity}

If $\mathrm{THz}$ radiation is irradiated onto a $\mathrm{CeCu}_{5.9} \mathrm{Au}_{0.1}$ single crystal, approximately $99.8 \%$ of the intensity is instantaneously reflected by the conduction electrons. The reflectivity of the conduction electrons can be extracted by measuring the intensity of the conduction electron response, between -3 and 3 ps. Integrating the $\mathrm{THz}$ pulse intensity between 3 and 8 ps allows us to specify the intensity captured in the Kondo reflex. It turns out that only a mere $1.6 \%$ of the T-ray intensity is involved in the heavy-fermion dynamics, see Fig. 6.10. Furthermore, the Kondo-echo intensity scales linearly with the incident $\mathrm{THz}$ intensity.
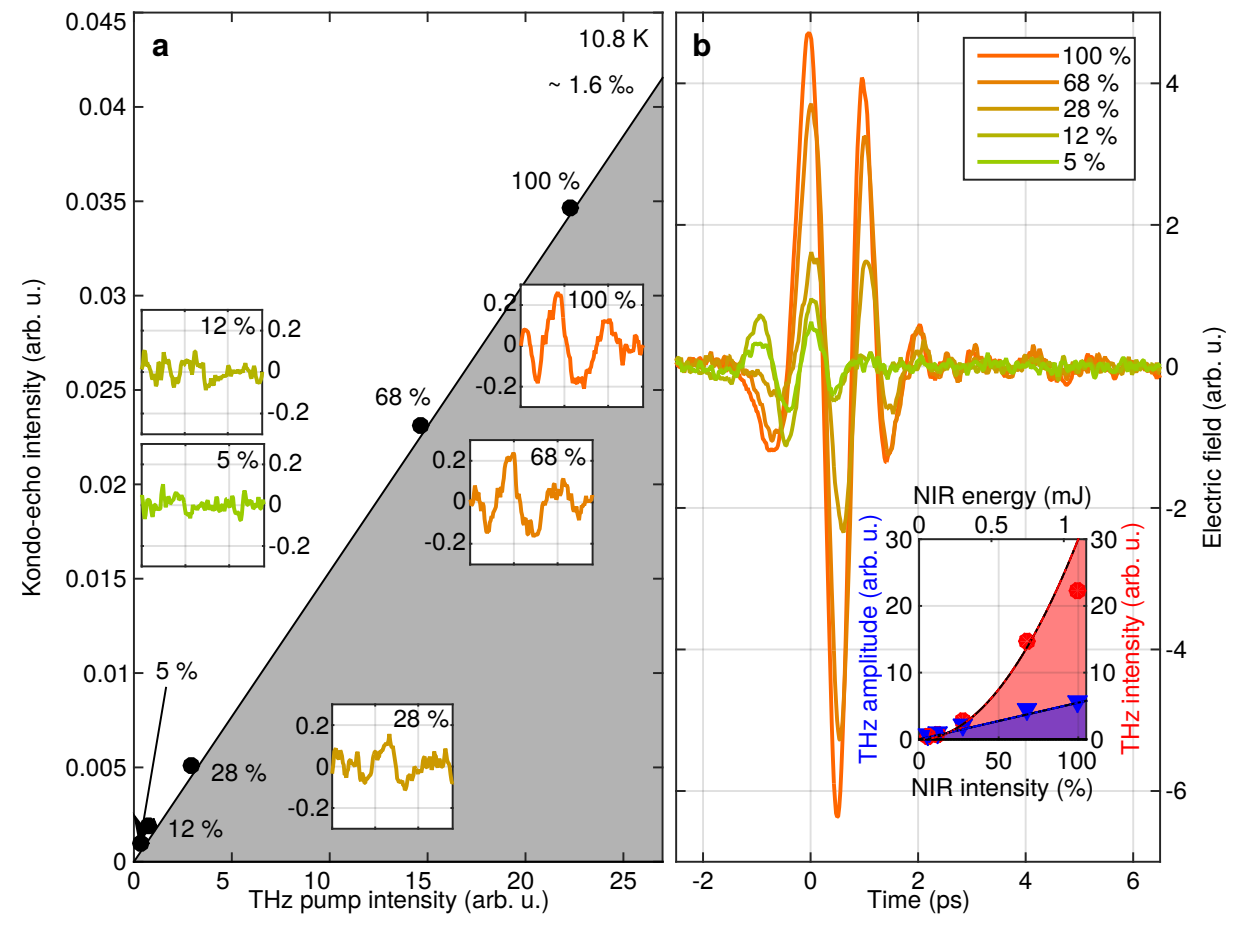

Figure 6.10.: (a) Kondo-echo intensity as function of the incident $\mathrm{THz}$ pump intensity. The intensity integral between -3 and $3 \mathrm{ps}$ and between 3 and 8 ps directly reveal the ratio between radiation reflected by the conduction electrons and the heavy-fermion process, respectively. The Kondo-echo intensity scales linearly with the $\mathrm{THz}$ pump intensity (black dots). The insets show the raw data of the Kondo echo as function of the relative $\mathrm{THz}$ pump intensity. The $\mathrm{THz}$ pump intensity is varied by reducing the NIR pump laser used to generate the $\mathrm{THz}$ radiation. (b) shows the collected raw data, where the percentage shows the relative NIR pump intensity. The inset shows that the $\mathrm{THz}$ amplitude scales linearly with the NIR pump intensity, whereas the $\mathrm{THz}$ intensity scales quadratically. The maximum NIR pump power available was $1.1 \mathrm{~mJ}$. For more details about the $\mathrm{THz}$ generation, see section 5.4 


\subsection{Frequency response in $\mathrm{CeCu}_{5.9} \mathrm{Au}_{0.1}$}

\subsubsection{Spectrum of full time trace}

The coherent dynamics in the quantum critical $\mathrm{CeCu}_{5.9} \mathrm{Au}_{0.1}$ compound has a distinctive signature in the time domain, see section 6.2. However, this unique heavy-fermion echo can be also investigated in the frequency domain, where some further features are revealed. Similar to the characteristic time delay $\tau_{\mathrm{K}}^{*}$, here a single frequency describes the spectral features related to the echo signature. This characteristic frequency again corresponds to the inverse effective Kondo temperature.

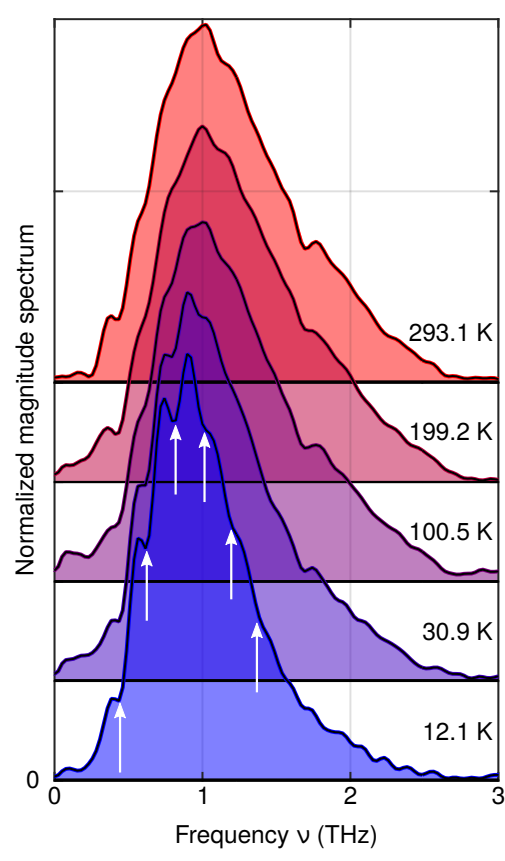

Figure 6.11: Fourier transformation of the intensity-normalized electric field time traces between -3 and $9 \mathrm{ps}$. These time traces include the conduction electron response as well as the temporally delayed heavy-fermion response. Hence, at sufficiently low temperatures the spectrum shows fringes (minima indicated by arrows). The periodicity of the fringes is $0.17 \mathrm{THz}$, which corresponds to the effective Kondo temperature, see Eq. 6.12.

The temperature-dependent Fourier transform of the full time trace (between -3 and $9 \mathrm{ps}$ ) includes the heavy-fermion echo (at $5.8 \mathrm{ps)}$ as well as the conduction response (at $0 \mathrm{ps}$ ). As a result, the spectrum shows interference fringes at sufficiently low temperatures, see Fig. 6.11. More details about how these fringes emerge are presented in appendix D.2. The amplitude of the fringes is temperature dependent as the Kondo echo increases with decreasing temperature. The periodicity of the fringes allow to directly extract the Kondo temperature:

$$
T_{\mathrm{K}}^{*}=\frac{h \nu}{k_{\mathrm{B}}} .
$$


However, for extracting the Kondo spectral weight the interference pattern has to be analyzed. Fig. 6.11 illustrates the difficulty of extracting a small signal from a large background, as this analysis method does not take advantage of the temporal shift between the conduction electron response and the heavy-fermion response. In a figurative sense, this illustrates the difficulty of characterizing Kondo systems using THz-FDS as also there the separation of the conduction electron response and heavy-fermion response is not given.

\subsubsection{Spectrum of the Kondo echo}

Due to the Kondo delay time, the Kondo echo of the $\mathrm{CeCu}_{5.9} \mathrm{Au}_{0.1}$ system is observed virtually background-free, see Fig. 6.2. Hence, by restricting the frequency analysis to the echo signal, the Kondo spectral weight and therefore the probability of the quasiparticle formation can be directly accessed. The intensity of the Kondo echo contains the Kondo spectral weight, see section 7.1.2. The echo, which emerging after a characteristic time scale $\tau_{\mathrm{K}}^{*}$, monitors the resurgence of the Kondo state, see Fig. 6.12.

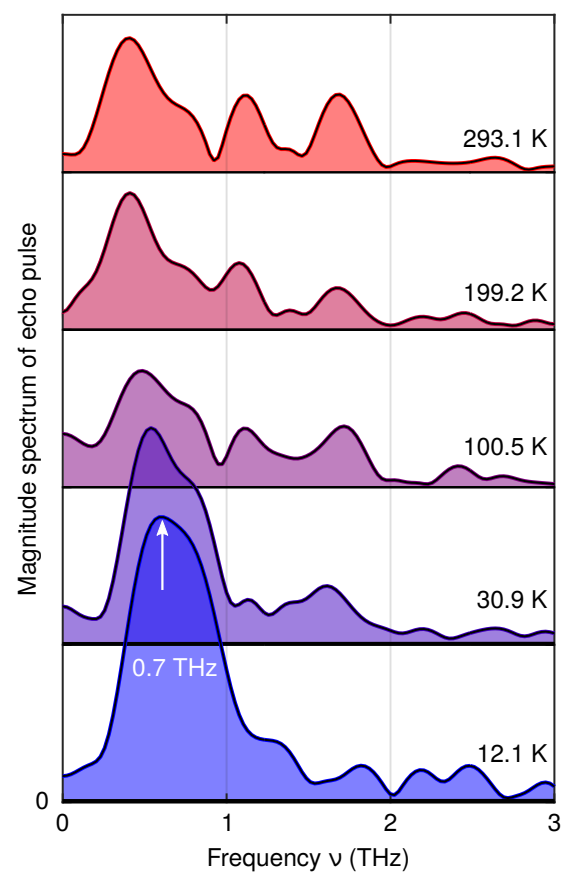

Figure 6.12: Temperature-dependent magnitude spectrum of the Kondo echo. The magnitude spectrum of the Kondo echo is calculated from the Kondo electric field pulse (time interval between 3 and $9 \mathrm{ps}$ ). The intensity of the electric field was a priori normalized on an interval between -3 and 9 ps. With decreasing temperature, the spectrum shows an increasing resonance centered at $0.7 \mathrm{THz}$ (indicated by the arrow). The central frequency of the echo signal is lowered compared to the incident $\mathrm{THz}$ pulse, since the envelope of the echo signal scales with the delay time $\tau_{\mathrm{K}}^{*}$, see Fig. 6.7. This leads to a lowering of the central frequency.

The ratio between the heavy and light electrons in the heavy-fermion system is directly accessed by the intensity ratio between the conduction electron and the echo pulse. As shown in Figs. 6.6 and 6.12 the intensity of the Kondo echo (and therefore the Kondo spectral weight) is temperature dependent. For a heavy-fermion system the spectral weight is expected to approach a constant value at sufficiently low temperatures. However, as the QCP is approached deviations 
from the anticipated behavior are expected. The low-temperature behavior of the echo signal and hence the quantum critical behavior is discussed in chapter 7 .

\subsubsection{Kondo response function}

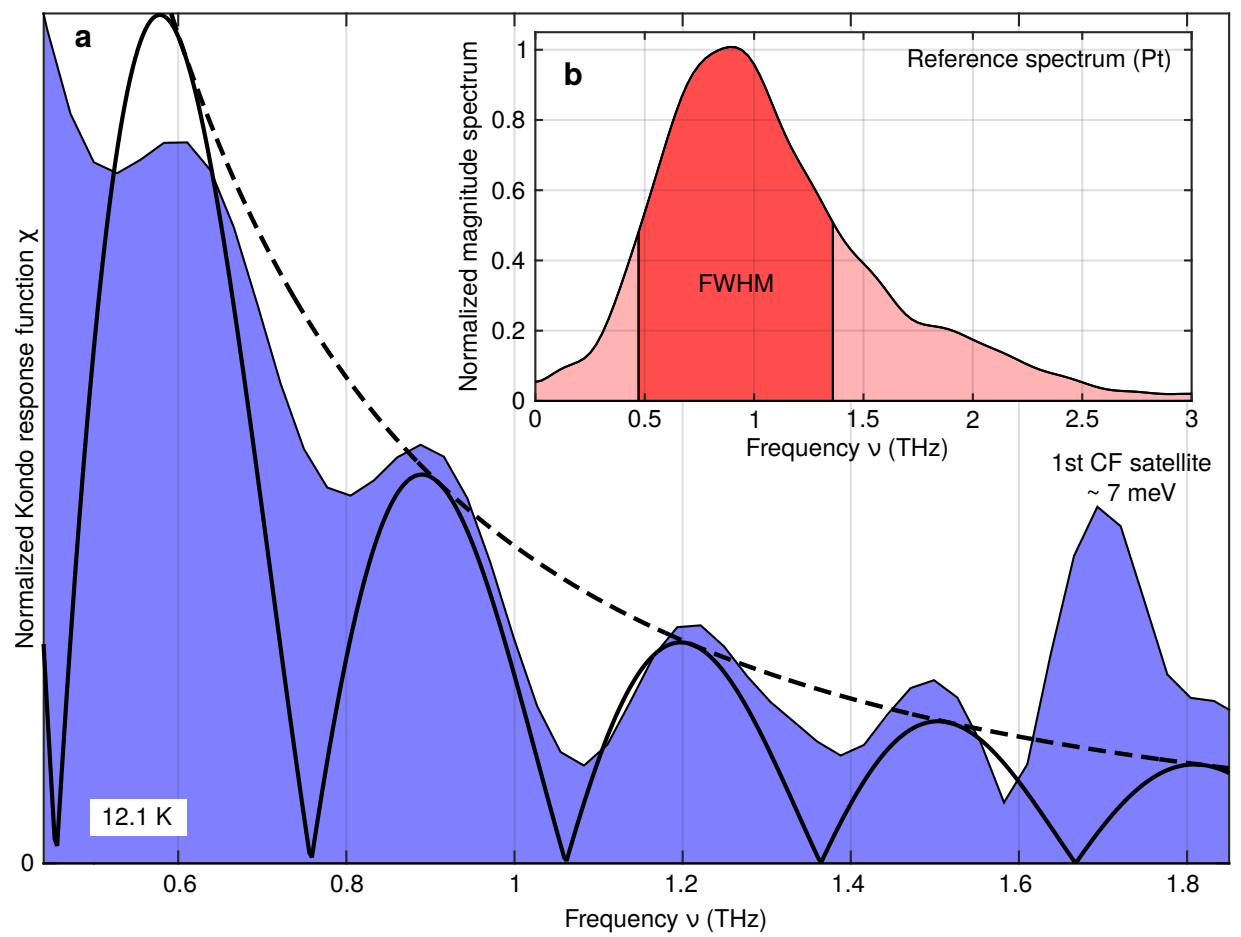

Figure 6.13.: The response function can be extracted by dividing the complex spectrum of the system response (between 3 and $9 \mathrm{ps}$ ) with the complex reference spectrum (platinum mirror between -3 and $3 \mathrm{ps}$ ). Inset (b) shows the reference magnitude spectrum of the platinum mirror. (a) The Kondo response function $\chi(\nu)$ (blue) shows periodic oscillations, with a Lorentzian envelope (dashed line). The periodic oscillations emerge due to the time delay of the echo signal. The black line illustrates the Lorentzian model function, described in Eq. 6.13. The only fitting parameter of this model is $\Gamma=0.193 \mathrm{THz}$, which corresponds to $T_{\mathrm{K}}^{*}=9 \mathrm{~K}$. Again, this is in good agreement with the Kondo temperature of $\mathrm{CeCu}_{5.9} \mathrm{Au}_{0.1}$. A deviation from the model function can be found at $1.7 \mathrm{THz}$. This signature energetically coincides with the first CF satellite of the measured compound 68, as described in section 2.7.1.

As illustrated in Fig. 6.8, the heavy-fermion response is dominated by the incident T-ray pulse shape. However, the main interest is in the bare material property and therefore the electromagnetic response function $\chi$ of the material, see section 5.7 . 
The resulting Kondo response function is illustrated in Fig. 6.13, where the frequency interval is restricted to a region of high $\mathrm{THz}$ intensities, as this reduces statistical errors.

The overall behavior of the measured electromagnetic response function, as shown in Fig. 6.13, can be represented by the following basic model function:

$$
\chi_{\text {model }}(\nu)=\frac{\Gamma^{2}}{\nu^{2}+\Gamma^{2}}\left|\cos \left(\frac{2 \nu}{\Gamma}\right)\right|
$$

This model function consists of a Lorentzian envelope as well as periodic oscillations. The model function has only one fitting parameter $\Gamma$. The measured electromagnetic response function is well represented by the model for a $\Gamma$ value of $0.193 \mathrm{THz}$ (error: $\pm 5 \mathrm{GHz}$ ), see black line in Fig. 6.13. This fitting parameter, which corresponds to $T_{\mathrm{K}}^{*}=9 \mathrm{~K}$, is again in good agreement with the Kondo temperature of the system investigated.

Note that deviations from the Lorentzian envelope are observed in the high-frequency range. A peak-like feature appears centered at a frequency of 1.7 THz which corresponds to $7 \mathrm{meV}$. The central frequency of the peak is energetically in excellent agreement with the first $\mathrm{CF}$ satellite of $\mathrm{CeCu}_{5.9} \mathrm{Au}_{0.1}$, which is reported to be around $8 \mathrm{meV}$ [68]. In other words, the temporally delayed pulse not only contains the heavy-fermion breakdown information but also information about the energetically shifted CF satellite peaks. This high-frequency signature is also theoretically confirmed by the DMFT calculations, see section 6.4.2.

\subsection{High-temperature Kondo effect in $\mathrm{CeCu}_{5.9} \mathrm{Au}_{0.1}$}

As explained in section 2.7.2 at intermediate temperatures a so-called high-temperature Kondo signature with a largely increased Kondo temperature $T_{\mathrm{K}}^{*,>}$ is expected to emerge. Due to thermal broadening of the Kondo resonance and its CF satellites, all the resonances broaden and eventually turn into a single, spectrally broad resonance, see section 2.7

The spectral width of this resonance defines the high-temperature effective Kondo temperature $T_{\mathrm{K}}^{*,>}$, which is in the order of the highest $\mathrm{CF}$ satellite level, see Fig. 2.10, In $\mathrm{CeCu}_{5.9} \mathrm{Au}_{0.1}$, the highest $\mathrm{CF}$ satellite is located $13 \mathrm{meV}$ above the Kondo resonance [68]. Correspondingly, the high-temperature Kondo temperature $T_{\mathrm{K}}^{*,>}$ is expected to be approximately $150 \mathrm{~K}$.

In terms of time delay, $T_{\mathrm{K}}^{*,>}$ corresponds to a $\tau_{\mathrm{K}}^{*,>}$ of approximately $320 \mathrm{fs}$. Hence, in the time domain a high-frequency oscillation is expected to emerge after $\tau_{\mathrm{K}}^{*,>}$ for intermediate temperatures. Indeed, the experiments are in good agreement with 
the scenario described above, see Fig.6.14. Because of the short time delay, a major overlap between the conduction response and the Kondo signature is expected.
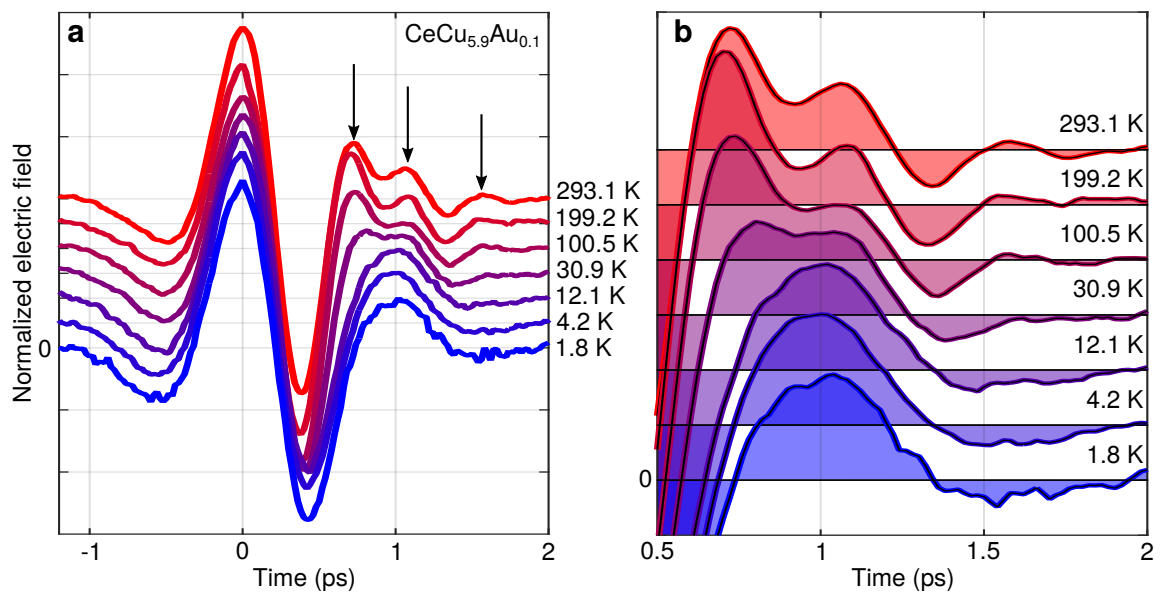

Figure 6.14.: (a) At high temperatures, a high-frequency oscillation with a short delay time is observed in the normalized electric field data (indicated by the arrows). The pulse intensities for all time traces were normalized on an interval between -3 and 9 ps. Since the delay time $\tau_{\mathrm{K}}^{*,>} \approx 320 \mathrm{fs}$ is shorter than the incident T-ray pulse, the high-temperature Kondo signature temporally overlaps with the conduction electron response. This high-frequency oscillation is dying as the sample is cooled and the echo pulse at $\tau_{\mathrm{K}}^{*} \approx 5.8 \mathrm{ps}$ is emerging, see Fig. 6.6. This feature reflects the emergence of the Kondo signature and its CF satellites into a single, spectrally broad resonance. The data imply a high-temperature Kondo scale in the range of $150 \mathrm{~K}$. (b) shows the electric field signal between 0.5 and $2 \mathrm{ps}$, where the high-frequency modulation at high temperatures (red) is prominent. At low temperatures (blue), the high-frequency oscillation disappears.

The values of the measurements shown in Fig. 6.14 are in excellent agreement with the expected values. Furthermore, the high-frequency oscillation is reduced as the sample is cooled down and eventually completely vanishes. At sufficiently low temperatures, the heavy-fermion bands (Kondo resonance and its satellites) are well separated, see section 2.7. The observation of $\tau_{\mathrm{K}}^{*,>}$ is yet another confirmation of the unique dynamics of heavy-fermion systems and a demonstration of the capability of THz-TDS. By Fourier analyzing the conduction electron response, which contains the high-temperature Kondo signature, it becomes obvious that the high-frequency part is reduced as the sample temperature is decreased.

The high-temperature Kondo spectral weight can be extracted in the frequency domain as a peak-like feature centered at approximately $2 \mathrm{THz}$. The temperature-dependent magnitudes of the measured spectra are shown in Fig. 6.15 . 

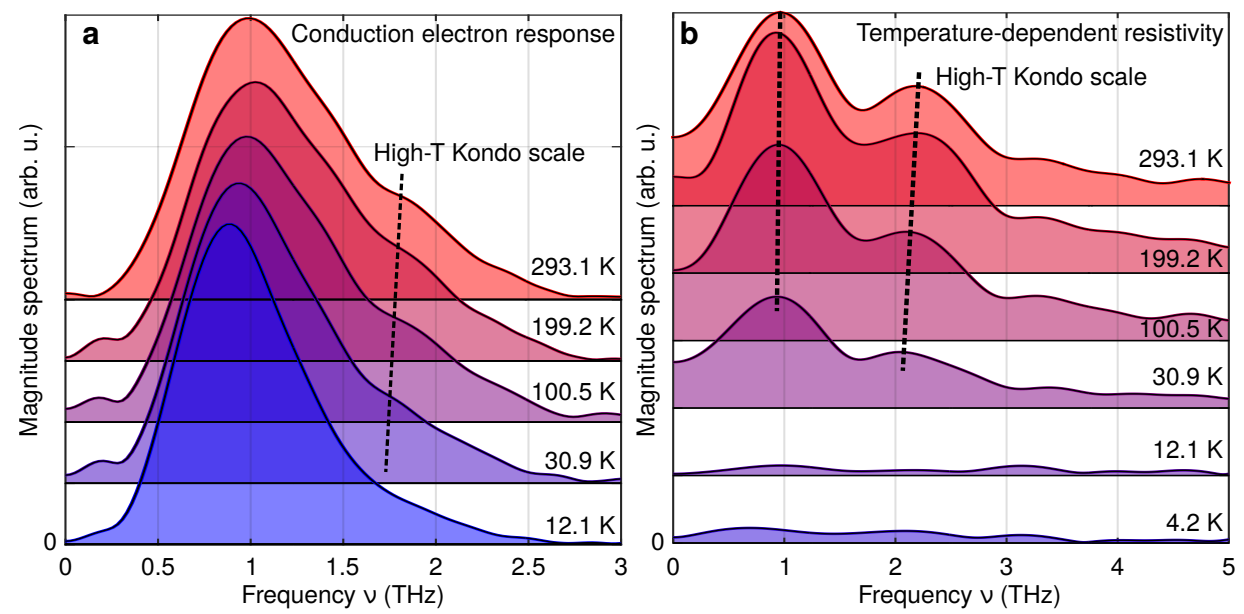

Figure 6.15.: The high-frequency oscillations shown in Fig. 6.14 also manifest in the magnitude spectra. (a) The spectrum of the normalized electric field signal (between -3 and $3 \mathrm{ps}$ ) shows a peak centered at $1 \mathrm{THz}$, which is caused by the conduction electrons. The high-frequency oscillations are seen as a weak side peak located at approximately $1.7 \mathrm{THz}$ (indicated by dashed line). (b) To reduce the conduction electron response, the normalized electric field of a low-temperature measurement is subtracted. The magnitude spectra of the corrected electric fields shows two pronounced peaks. The peak centered at $1 \mathrm{THz}$ corresponds to the temperature-dependent resistivity and the peak centered around $2 \mathrm{THz}$ depicts the high-frequency oscillation. The dashed lines are indicating the temperature-dependent peak position. Also here, the interval between -3 and 3 ps was analyzed.

Since the high-frequency modulation in the time traces disappears at low temperatures (see Fig. 6.14, the high-temperature Kondo effect signal can be extracted by taking the low-temperature measurement in the time domain as the conduction electron response, see Fig.6.14(a). As a consequence of this assumption the low-temperature time trace has to be subtracted from each time trace in order to reduce the influence of the conduction electrons. The resulting time trace can be converted to the frequency domain, see Fig. 6.15 (b). 


\subsection{Coherent heavy-fermion behavior in $\mathrm{YbRh}_{2} \mathrm{Si}_{2}$}

$\mathrm{YbRh}_{2} \mathrm{Si}_{2}$ is another well-studied heavy-fermion compound which can be driven with a small magnetic field or with Ge doping into the quantum critical regime, see section 4.2. The Kondo temperature for $\mathrm{YbRh}_{2} \mathrm{Si}_{2}$ is reported to be approximately $25 \mathrm{~K}$ [54]. Hence, a corresponding delay time of $\tau_{\mathrm{K}}^{*}=1.9 \mathrm{ps}$ is expected. Hence, the Kondo echo will overlap with the conduction electron response. Indeed, a temperature-dependent feature is observed see Fig. 6.16.
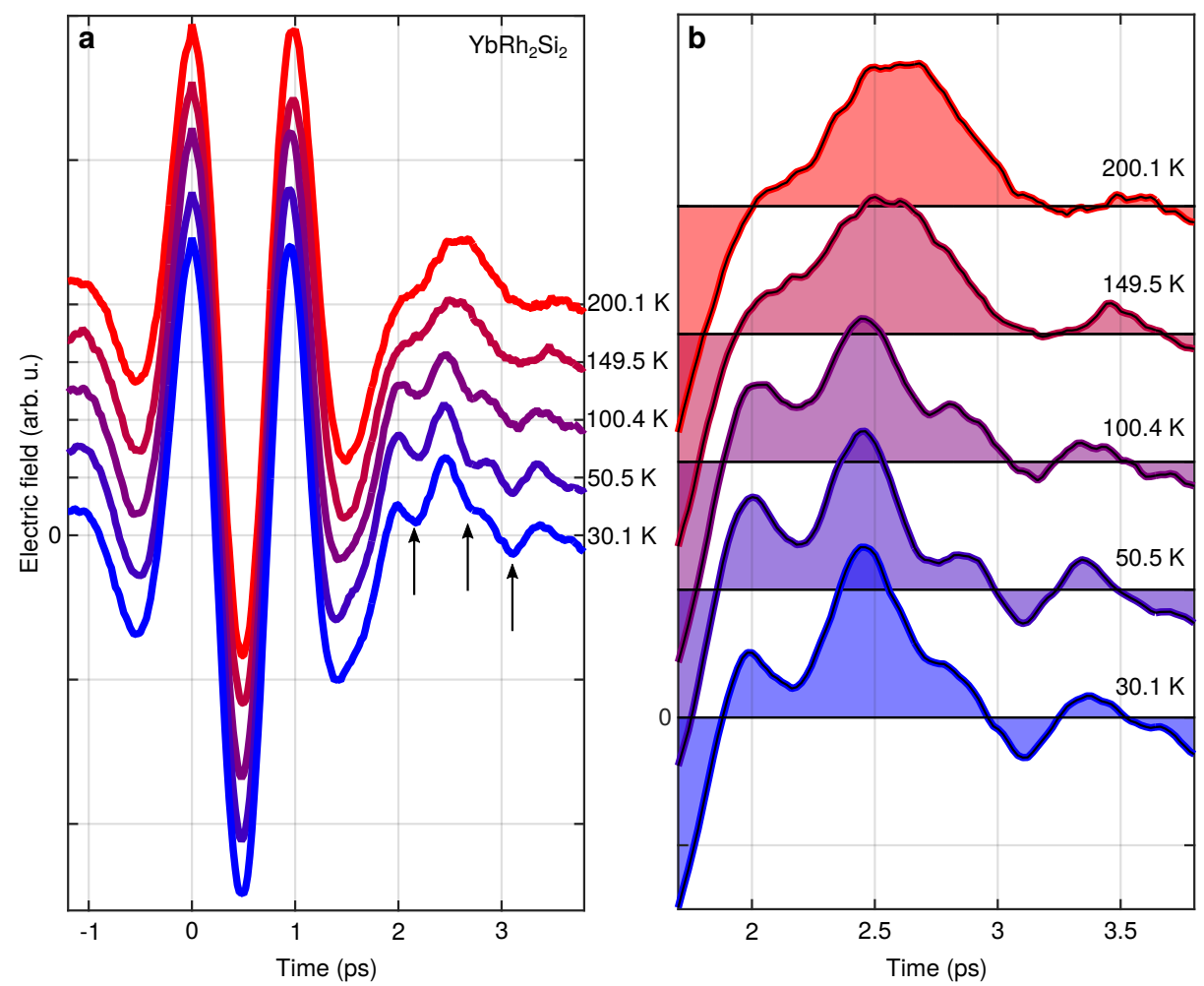

Figure 6.16.: (a) Temperature-dependent electric field traces reflected on a $\mathrm{YbRh}_{2} \mathrm{Si}_{2}$ single crystal. For each data set, the pulse intensity is normalized on an interval between -3 and 6 ps. As the sample temperature is decreased, an additional oscillation between $2-3$ ps emerges (indicated by the arrows). From this observation, a Kondo response time $\tau_{\mathrm{K}}^{*} \approx 2 \mathrm{ps}$ is extracted, which corresponds to an effective Kondo temperature $T_{\mathrm{K}}^{*}=24 \mathrm{~K}$. It is in good agreement with the reported Kondo temperature of the system [54]. (b) shows the same data between 1.7 and $3.7 \mathrm{ps}$. Oscillations emerge as the temperature is decreased. 
Because of the relatively high Kondo temperature $T_{\mathrm{K}}^{*}$, the heavy-fermion signal is no longer background-free, making the analysis a lot more complex and the feature less striking than for the $\mathrm{CeCu}_{5.9} \mathrm{Au}_{0.1}$ sample, see the previous discussion. Nevertheless, the fact that a very similar echo response is also observed in this heavy-fermion compound is a substantial confirmation of this novel approach towards heavy-fermion dynamics. This confirmation is not only qualitatively correct but also numerically all the experimental observations coincide well with literature values as well as the suggested models. The conduction electron background also has some profound effects on the frequency domain. In Fig. 6.17 the frequency domain is calculated for the electric field time trace of the $\mathrm{THz}$ response between 1.5 and $4.5 \mathrm{ps}$.

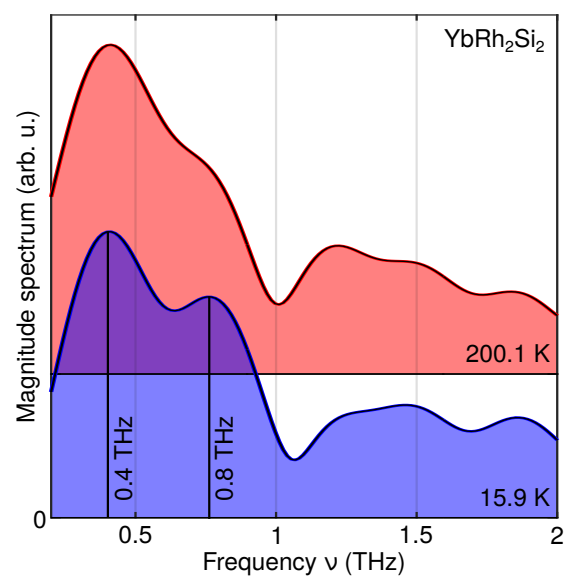

Figure 6.17: The emerging oscillation also manifests in the magnitude spectrum. The normalized electric field between 1.5 and $4.5 \mathrm{ps}$ (see Fig. 6.16 is used for the spectral analysis. As the sample temperature is reduced (blue), the emerging Kondo signal interferes with the background signal. Similar to the linear response analysis in $\mathrm{CeCu}_{5.9} \mathrm{Au}_{0.1}$, the fringe pattern allows us to conclude on the Kondo temperature. The peak-to-peak distance at low temperatures corresponds to $0.4 \mathrm{THz}$, which corresponds to a Kondo temperature of $T_{\mathrm{K}}^{*}=20 \mathrm{~K}$.

In contrast to the $\mathrm{CeCu}_{5.9} \mathrm{Au}_{0.1}$ compound, the background signal at high temperatures is not negligible due to the conduction electron background. However, as the temperature is further reduced, a signature with a periodicity of approximately $0.4 \mathrm{THz}$ emerges, which corresponds to a $2.5 \mathrm{ps}$ time-delayed echo, as estimated from Fig. 6.16. Hence, also the frequency-domain analysis suggests an effective Kondo temperature $T_{\mathrm{K}}^{*}=20 \mathrm{~K}$, which is also in good agreement with the literature value of $25 \mathrm{~K}$ for undoped $\mathrm{YbRh}_{2} \mathrm{Si}_{2}$ [54. Therefore, the two quantities delay time $\tau_{\mathrm{K}}^{*}$ and the oscillation in frequency correctly indicate the Kondo temperature of the system.

For investigating quantum critical behavior an external magnetic field of $0.7 \mathrm{~T}$ has to be applied [105]. Already at this point, the fact that the echo signals are observed for two distinctively different heavy-fermion systems $\left(\mathrm{CeCu}_{5.9} \mathrm{Au}_{0.1}\right.$ and $\mathrm{YbRh}_{2} \mathrm{Si}_{2}$ ), one of which is quantum critical and the other one being well within the heavy-fermion phase, constitute a strong confirmation that the explanations presented here correctly describe the correlation dynamics of heavy-fermion systems. 



\section{Quantum phase transition in heavy fermion $\mathrm{CeCu}_{5.9} \mathrm{Au}_{0.1}$}

Quantum phase transitions (QPTs) are highly interesting as they appear at $0 \mathrm{~K}$ and are purely driven by quantum-mechanical fluctuations 1 In fact, the interest in QPTs was boosted by the discovery of a non-Fermi-liquid behavior [10, 13, as well as the trailblazing discovery of superconductivity in magnetic systems in its immediate vicinity [2, 3, 15, 33].

The ability to observe the strong correlation dynamics turns out to be also very useful to investigate the quantum critical regime, as it depicts an effective way of monitoring heavy-fermion states. T-rays are well suited for time-resolved studies of heavy-fermion behavior and consequently also to directly observe non-Fermi-liquid behavior near QPTs. As shown in chapter 6, a short T-ray pulse can be used to coherently excite the electrons in a heavy-fermion ground state. The $\mathrm{THz}$ radiation efficiently disintegrates the Kondo singlets. Since the large effective mass is a direct consequence of strong correlation, the reformation of the heavy band is time delayed by the unique dynamics of heavy fermions, see section 6.4 . This allows us to perform a background-free analysis of the heavy-fermion state, which makes THz-TDS an excellent tool for investigating quantum criticality.

As described in chapter 3 the two existing quantum critical scenarios have some distinct differences concerning the behavior of Kondo singlets as the QPT is approached [12]. In the conventional quantum critical scenario, the non-Fermi-liquid behavior is caused by a spin-density wave (SDW) ground state, which causes a partial collapse of the Fermi surface (hybridization gap at $E_{\mathrm{F}}$ ). However, since the the Fermi surface only collapses along the nesting vector of the SDW, Kondo singlets can still coexist on other regions of the Fermi surface, leading to a finite Kondo weight, even at vanishingly small temperatures. However, $\mathrm{CeCu}_{5.9} \mathrm{Au}_{0.1}$ is known to be an unconventional quantum critical system (see chapter 4) where an entire Fermi-surface collapse is predicted. This, also called localized quantum critical scenario, leads to a destruction of all Kondo singlets which is commonly interpreted to conform with a vanishing Kondo temperature as the QPT is approached [12, 35, 82,84. However, the work at hand demonstrates that the Kondo weight collapse is completely decoupled from the effective Kondo temperature. In fact, the Kondo spectral weight fully collapses (below $\sim 5 \mathrm{~K}$ ), while the Kondo temperature remains finite to the lowest accessible temperature, which

${ }^{1}$ In contrast, phase transitions with finite transition temperatures are always dominated by thermal fluctuations. 
is $1.8 \mathrm{~K}$. These results could change our established view of quantum criticality and allow for first insights into its dynamical aspects.

\subsection{Kondo-echo analysis}

The temperature-dependent T-ray time traces measured on an unconventional quantum critical $\mathrm{CeCu}_{5.9} \mathrm{Au}_{0.1}$ single crystal reveal two distinct features of the heavy-fermion ground state: the Kondo spectral weight as well as the effective Kondo temperature. The delay time corresponds to the inverse Kondo temperature $T_{\mathrm{K}}^{*}$ and the intensity of the echo signature contains information about the amount of Kondo singlets involved in the observed dynamical process. The great advantage of the time-resolved $\mathrm{THz}$ ansatz is the simultaneous monitoring of both quantities.

It should be mentioned that an echo pulse can be observed throughout the temperature range investigated. However, the intensity is strongly temperature dependent. Also the Kondo energy scale shows a temperature dependence. This fact, in combination with the models and reference measurements, is a solid proof that the delayed echo pulse is caused by the coherent strong correlation dynamics. In fact, the high-temperature measurements can be used to estimate incoherent background when extracting the Kondo spectral weight, as illustrated in section 7.1 .2

\subsubsection{Temperature dependence of the Kondo scale $T_{\mathrm{K}}^{*}$}

For monitoring the temperature-dependent Kondo scale $T_{\mathrm{K}}^{*}$, the echo position $\tau_{\mathrm{K}}^{*}$ has to be measured as function of the sample temperature. The position of the echo signal is always approximately at $6 \mathrm{ps}$. However, an increase in the temporal gap between the conduction electron response and the echo signal can be observed. The drift can be illustrated by normalizing the Kondo reflex intensity 2 Then, Fig. 7.1 reveals a drift in $\tau_{\mathrm{K}}^{*}$ towards larger values as the sample is cooled. Hence, this corresponds to a decrease of the Kondo temperature $T_{\mathrm{K}}^{*}$. Furthermore, this dilatation of the echo pulse corresponds to a decreasing width of the heavy-fermion spectral weight, as the system approaches the QCP.

The temperature dependence of the Kondo energy scale revealed by Fig. 7.1 is striking. In fact, in the established unconventional quantum critical model the Kondo temperature $T_{\mathrm{K}}^{*}$ is expected to vanish as the QCP is approached. However, only a minor drift of the echo is observed. The temperature-dependent delay time $\tau_{\mathrm{K}}^{*}$ can be translated into a temperature-dependent Kondo scale $T_{\mathrm{K}}^{*}$, see Eq. 6.11 and Fig. 7.3 .

The relative time between conduction electron response and the strong correlation Kondo response can be measured with a temporal precision of less than $100 \mathrm{fs}$.

${ }^{2}$ The Kondo reflex is rescaled such that the integrated Kondo-echo intensity between 3 and 8 ps is 1 . 


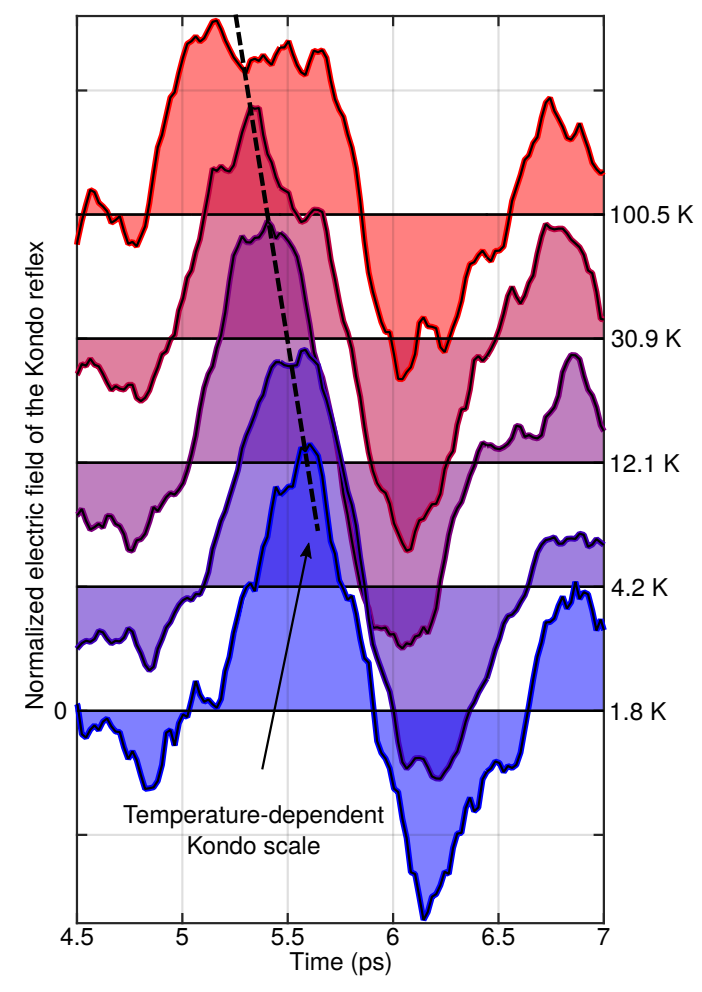

Figure 7.1: The normalized Kondo echo illustrates that the Kondo time delay $\tau_{\mathrm{K}}^{*}$ is actually slightly temperature dependent. All displayed curves are normalized to the same integrated intensity between 3 and 8 ps. Hence, this data reveals how $T_{\mathrm{K}}^{*}$ changes with temperature as its inverse specifies the time scale $\tau_{\mathrm{K}}^{*}$, on which the quasiparticles are formed. Here, the spectral weight (i.e. the probability of quasiparticle formation) is normalized to 1 .

The scattering of the data points in Fig. 7.3 (b) is of the order of $0.1 \mathrm{~K}$, which corresponds to a frequency resolution of just $2 \mathrm{GHz}$, which is on the edge of detectability with the setup built by the author. Nevertheless, the trend at lower temperatures is obvious and clearly distinguishable from the noise level. In fact, a decrease of the Kondo temperature is expected for unconventional quantum criticality. However, the quantum critical behavior also suggests a complete extinction of the Kondo temperature scale $T_{\mathrm{K}}^{*}$ as the QPT is approached and Kondo spectral weight collapses. Since the experiment is limited to temperatures above $1.8 \mathrm{~K}$, extracting the $0 \mathrm{~K}$ behavior is ambiguous. Nevertheless, Fig. 7.3 shows a reasonable linear extension from the higher temperatures.

\subsubsection{Temperature-dependent Kondo spectral weight}

As mentioned, THz-TDS allows us to separately measure the Kondo spectral weight as well as the Kondo temperature. As the quantum critical regime is reached for sufficiently low temperatures, the Kondo spectral weight is drastically reduced, see Fig. 7.3. This fate of the quasiparticles is of major interest since it reveals the effects of quantum criticality. 


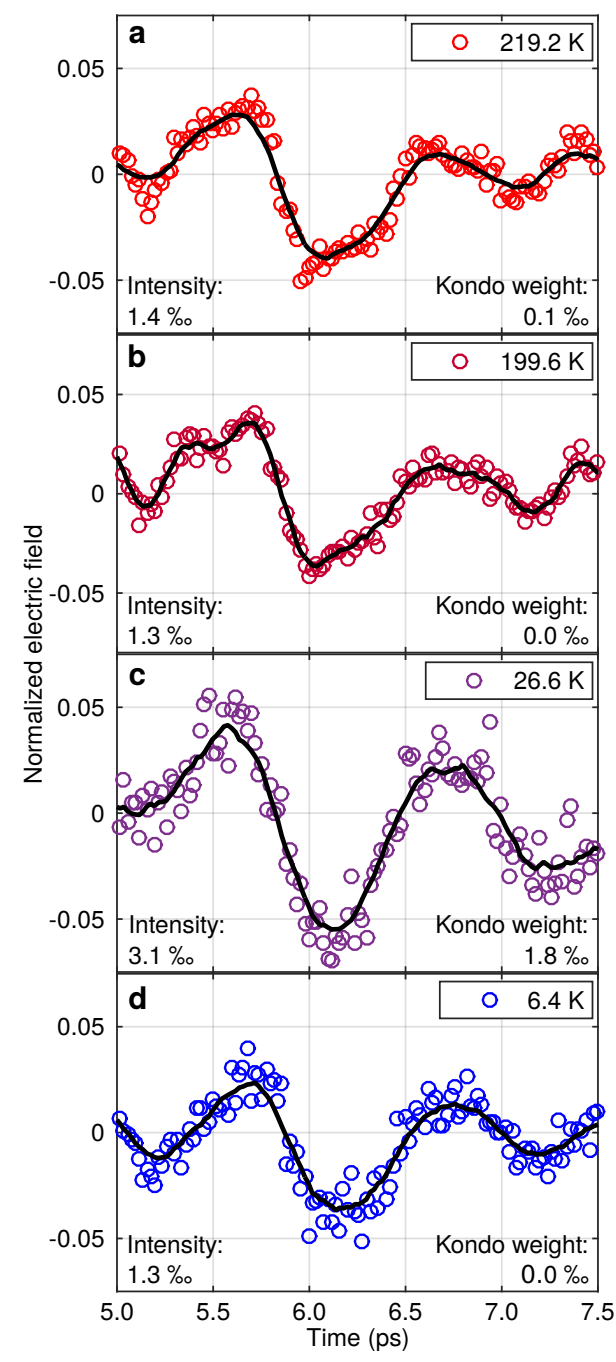

Figure 7.2: Temperature-dependent echo signal in the normalized electric field data $(\mathrm{THz}$ intensity normalized between -2 and $8 \mathrm{ps}$ ). The echo intensity (integrated between 3 and $7.5 \mathrm{ps}$ ) at $T=219.2 \mathrm{~K}$ (a), $T=199.6 \mathrm{~K}(\mathrm{~b})$ and $T=6.4 \mathrm{~K}$ (d) are almost identical. This value characterizes the spin-correlation background, which is not related to the heavy-fermion quasiparticle weight. Hence, the Kondo weight corresponds to the integrated echo intensity, after the subtraction of this spin-correlation background. (c) shows the echo pulse at $T=26.6 \mathrm{~K}$, which contains a larger intensity $\left(3.1 \cdot 10^{-3}\right)$. After the subtraction of background, a ,pure ${ }^{6}$ Kondo weight of $1.8 \cdot 10^{-3}$ remains. The full temperature dependence is shown in Fig. 7.3. The resulting Kondo weight peaks around $25 \mathrm{~K}$. The black line illustrates the data after it was smoothened by a moving average over 5 data points. For more details, see text.

Note that the electric field signals of the $\mathrm{CeCu}_{5.9} \mathrm{Au}_{0.1}$ show a small echo pulse throughout the temperature range (between 1.8 and $300 \mathrm{~K}$ ), even outside of the heavy Fermi-liquid phase. This small echo signature is caused by the ,Kondo-like' spin correlations, which are not related to the heavy-fermion quasiparticle weight. Nevertheless, these spin correlations still contain the Kondo scale $T_{\mathrm{K}}^{*}$ 36. In the time-resolved data, these ,Kondo-like' spin correlations appear as time-delayed background features, which might be even faintly visible in the $\mathrm{CeCu}_{5.0} \mathrm{Au}_{1.0}$ compound in Fig. 6.1. Due to the existence of these spin correlations, the observation of the Kondo energy scale $T_{\mathrm{K}}^{*}$ is completely decoupled from the observation of the Kondo weight. In order to extract the correct Kondo weight, the ,Kondo-like' spin correlations have to be subtracted. Hence, the information of the Kondo spectral weight is hidden in temperature dependence of the intensity of the echo signal, see Fig. 7.2 . 
The Kondo spectral weight corresponds to the probability for the very existence of heavy quasiparticles. The Kondo temperature on the other hand characterizes the time scale, on which the heavy quasiparticles are formed. In fact, the unconventional quantum criticality causes the Kondo weight to eventually fully collapse, see Fig. 7.3. The T-ray experiment reveals that the Kondo spectral weight has a maximum around $25 \mathrm{~K}$. Below this threshold, the Kondo spectral weight quickly drops to the same level it has for sample temperatures above $200 \mathrm{~K}$, where heavy-fermion quasiparticles do not exist anymore. Hence, below $5 \mathrm{~K}$ the Kondo spectral weight is zero and remains constant towards even lower temperatures. These two aspects mark the complete spectral weight collapse of the Kondo effect. However, the simultaneously measured Kondo temperature $T_{\mathrm{K}}^{*}$ remains finite throughout the entire measurements, see Fig. 7.3 .

Fig. 7.3 reveals that while the quasiparticle spectral weight collapses due to unconventional quantum criticality, the Kondo temperature remains nearly constant. This observation is contrary to common belief, where also a Kondo temperature collapse is expected as the QCP is approached. These experimental results are unambiguously demonstrating that the two aspects are not contradictory as both quantities are recorded in a single $\mathrm{THz}$ experiment. Furthermore, they are consistent with the $E / T$ scaling behavior, a strong indicator of unconventional quantum criticality, as discussed in section 4.3.2. Hence, the data presented here calls for a revised view on heavy fermions near QCPs. More generally, the concept of quasiparticles in the quantum critical regime ought to be also revised. 


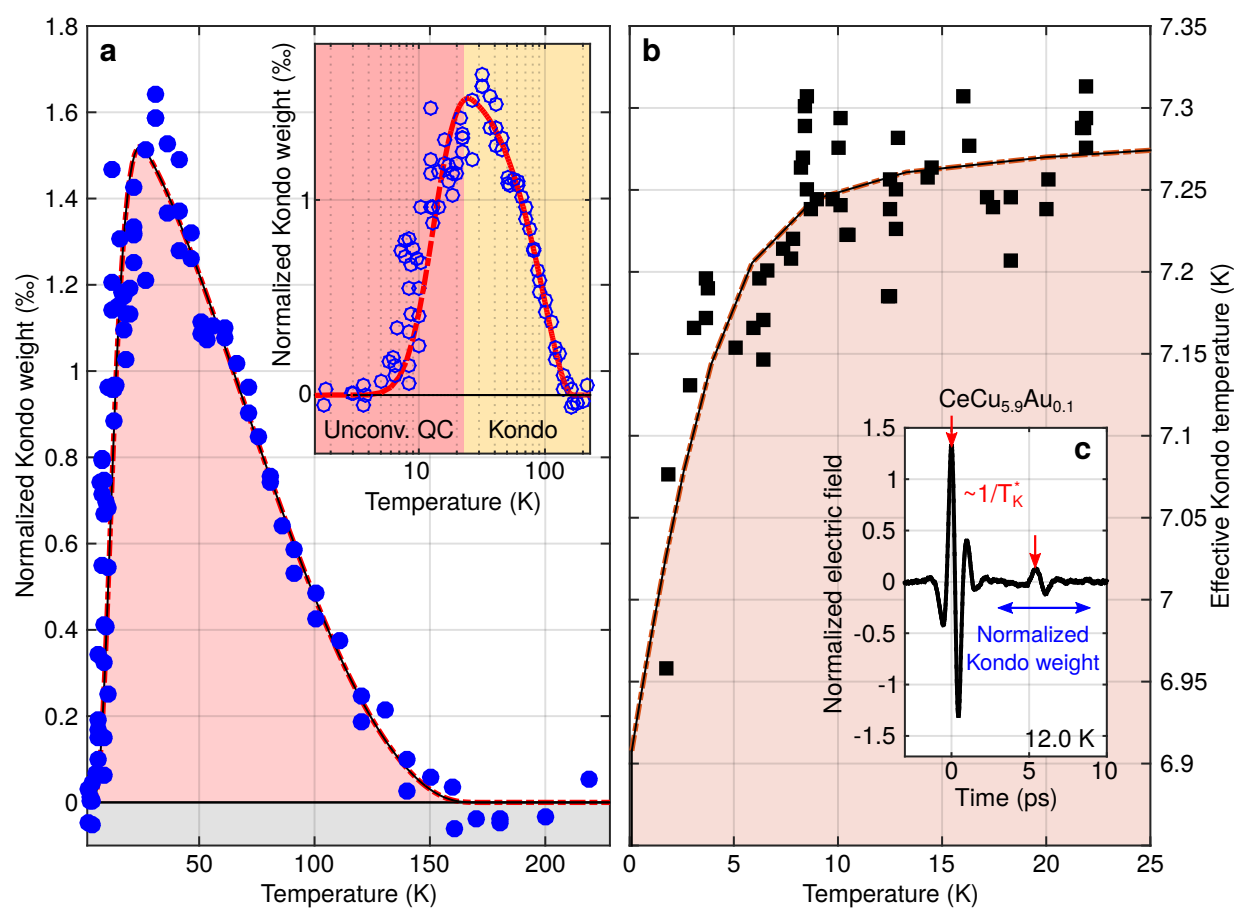

Figure 7.3.: (a) The Kondo spectral weight as function of sample temperature. With decreasing temperature, the spectral weight grows as expected for a Kondo system, before eventually fully collapsing again due to the proximity of the QCP. The Kondo weight below $\sim 5 \mathrm{~K}$ is equal to the Kondo weight above $200 \mathrm{~K}$ (for details on the offset correction, see text). The inset shows the same set of data points on a logarithmic scale. (b) The corresponding Kondo temperature $T_{\mathrm{K}}^{*}$ remains almost unchanged throughout the experiment. Only for temperatures below approximately $10 \mathrm{~K}$, the Kondo temperature starts to systematically decrease. Remarkably, the Kondo temperature remains finite, even when the Kondo spectral weight fully collapsed. Inset (c) shows a typical $\mathrm{THz}$ electric field pulse, where the pulse intensity between -3 and $8 \mathrm{ps}$ is normalized. The Kondo temperature is gained from the time delay of the maximum between the conduction electron response and the Kondo echo (indicated by the red arrows). The Kondo weight is gained from the intensity integral between 3 and 8 ps (indicated by the blue arrow). Remark: To improve the signal-to-noise ratio, the displayed data has been smoothened by a moving average over 5 data points. 


\section{Conclusion and outlook}

The development of powerful table-top terahertz sources over the last decades opened up the vast field of optical spectroscopy in the meV photon range [132, 169]. $\mathrm{THz}$ radiation is broadly used to investigate semiconductor structures, metallic thin films as well as strongly correlated systems. The optical properties of these systems have been intensively investigated and used to characterize complex structures, even on a sub-meV energy scale [37, 174, 175]. However, most optical experiments carried out on strongly correlated systems are performed using tunable continuous-wave sources such as backward-wave oscillators. This high-resolution optical spectroscopy allows for a very precise description of the equilibrium situation. However, no insights are gained about the non-equilibrium dynamics of strong correlation systems [156.

The thesis at hand is addressing the time resolution in strong correlation systems. THz-TDS allows us not only to gain spectroscopic information but also to observe the real-time dynamics. Heavy fermions are an excellent test system for time-resolved investigations, since the relevant energy scales of the underlying Kondo effect are reported to be in the order of $10 \mathrm{~K}$ [67]. This energetically coincides with the lower $\mathrm{THz}$ range of the electromagnetic spectrum. The intrinsic energy scale of the Kondo effect is called Kondo temperature $T_{\mathrm{K}}$. Since the inverse effective Kondo temperature $1 / T_{\mathrm{K}}^{*}$ defines the lifetime of the quasiparticles as well as the time scale on which strong correlation emerges, $\mathrm{CeCu}_{6-\mathrm{x}} \mathrm{Au}_{\mathrm{x}}$ compounds are expected to have exceptionally long-lived quasiparticles. Furthermore, $\mathrm{CeCu}_{6-\mathrm{x}} \mathrm{Au}_{\mathrm{x}}$ single crystals can be tuned to undergo a quantum phase transition (QPT) for a critical Au concentration. The QPT in this system is reported to be of unconventional nature [12]. The unconventional QCP makes the system particularly interesting for time-resolved $\mathrm{THz}$ experiments since an entirely new perspective towards quantum criticality is provided by this approach. This novel ansatz gives also direct access to the Kondo spectral weight as well as the effective Kondo temperature. Therefore, THz-TDS is also a viable approach to investigate heavy-fermion quasiparticles near the QCP. The totally different time scales of strongly and weakly correlated systems allow us to effectively unravel the two contributions giving a virtually background-free point of view on strong correlation dynamics. Furthermore, $\mathrm{THz}$ reflectivity measurements are not only compatible with low temperatures but also with externally applied magnetic fields.

The key results of this thesis can be summarized as follows: The time-resolved $\mathrm{THz}$ spectroscopy approach allows us to monitor temporally resolved the formation process of the strongly correlated Kondo ground state, while the system is tuned 
toward the quantum critical regime. The T-ray pulse is used to break up the strongly correlated ground state and therefore reduces the Kondo spectral weight at the Fermi surface. While the strong correlation re-establishes, the electrons are emitting a coherent echo signal in the $\mathrm{THz}$ range. Remarkably, the excited electrons are not dephasing on the time scale of picoseconds and hence a well preserved echo is observed. The temporal separation of the Kondo signature and the conduction electron response reveals not only the Kondo spectral weight accumulated at the Fermi surface, but simultaneously gives a direct access to the effective Kondo temperature (via the time delay of the response). The direct observation of both quantities, the Kondo spectral weight and the Kondo temperature, gives an unrivaled insight into heavy-fermion systems. Furthermore, it can be used to investigate non-Fermi-liquid behavior in the vicinity of QPTs. The experiment presented in this thesis demonstrated that with the onset of the unconventional quantum critical scenario the Kondo spectral weight is suppressed and vanishes below a critical temperature. However, in contrast to prevalent models, the Kondo temperature remains finite and nearly unchanged, even when the Kondo spectral weight is fully suppressed. The $\mathrm{THz}$ experiment further indicates the onset of Kondo-lattice coherence at temperatures far lower than the temperature range, where the spectral weight has fully collapsed due to the unconventional quantum criticality.

From an experimental point of view, the major difficulty was the fact that single crystals (rather than thin films on a substrate) were investigated. Due to the high reflectivity of metallic systems, the experimental data presented in this thesis were exclusively collected in reflection geometry.

The thesis successfully demonstrated that THz-TDS is a powerful tool to investigate Kondo-lattice dynamics and to get unrivaled insights into QPTs. Since time-resolved $\mathrm{THz}$ spectroscopy is an accurate method which most likely allows us to uncover many new aspects of heavy-fermion systems, the following aspects could be experimentally further addressed: Since the Kondo-lattice effect is of magnetic origin, externally applied magnetic field experiments could unravel new aspects of heavy fermions as well as the quantum criticality. Magnetic fields could not only be used to carefully tune systems into a quantum critical regime, but also to modify the type of the emerging quantum criticality from conventional to unconventional and vice-versa 12 .

Another interesting aspect which should be addressed, are Kondo-lattice coherence effects. The heavy-fermion compound $\mathrm{CeCu}_{6}$ presented here is reported to have a distinctive lattice coherence. However, by La-doping, the lattice coherence can be tuned while the on-site Kondo effect remains unaffected (radii of La and $\mathrm{Ce}$ are very similar). The coherence breakdown in $\mathrm{Ce}_{\mathrm{x}} \mathrm{La}_{1-\mathrm{x}} \mathrm{Cu}_{6}$ compounds is reported to occur for Ce concentrations smaller than $x=0.75$ [8, 198, 199]. Hence, $\mathrm{Ce}_{\mathrm{x}} \mathrm{La}_{1-\mathrm{x}} \mathrm{Cu}_{6}$ would allow us to gradually tune a Kondo lattice into a Kondo-impurity system.

Furthermore, photodoping aspects of heavy-fermion system could and should be addressed with the setup built by the author. This tempting ansatz (for instance 
of two-dimensional spectroscopy) could potentially allow us to transiently change the Kondo ground state [200 202].

Apart from heavy fermions and QPTs, T-rays and especially THz-TDS could play a key role in understanding further, highly complex strong correlation physics found in Mott insulators, high- $T_{\mathrm{c}}$ superconductors, but also in topologically protected states. Indeed, many groups around the globe discovered this promising approach and try to understand strongly correlated effects in solid state physics. Furthermore, experiments have shown that strongly correlated states can be induced using photodoping, which accentuates the relevance of understanding the (ultrafast) dynamics of strongly correlated ground states [134, 203,205]. 



\section{Bibliography}

[1] K. Andres, J. E. Graebner, and H. R. Ott, Phys. Rev. Lett. 35, 1779 (1975).

[2] F. Steglich, J. Aarts, C. D. Bredl, W. Lieke, D. Meschede, W. Franz, and H. Schäfer, Phys. Rev. Lett. 43, 1892 (1979).

[3] H. R. Ott, H. Rudigier, Z. Fisk, and J. L. Smith, Phys. Rev. Lett. 50, 1595 (1983).

[4] H. R. Ott, H. Rudigier, T. M. Rice, K. Ueda, Z. Fisk, and J. L. Sмiтh, Phys. Rev. Lett. 52, 1915 (1984).

[5] G. R. Stewart, Z. Fisk, and M. S. Wire, Phys. Rev. B 30, 482 (1984).

[6] D. E. Maclaughlin, C. Tien, W. G. Clark, M. D. Lan, Z. Fisk, J. L. Smith, and H. R. Oтt, Phys. Rev. Lett. 53, 1833 (1984).

[7] Y. Ōnuki, K. Shibutani, T. Hirai, T. Komatsubara, A. Sumiyama, Y. Oda, H. Nagano, H. Sato, and K. Yonemitsu, J. Phys. Soc. Jpn. 54, 2804 (1985).

[8] Y. Ånuki, Y. Shimizu, M. Nishinara, Y. Machi, and T. Komatsubara, J. Phys. Soc. Jpn. 54, 1964 (1985).

[9] H. R. Ott, H. Rudigier, Z. Fisk, and J. L. Smith, Phys. Rev. B 31, 1651 (1985).

[10] H. v. Löhneysen, A. Rosch, M. Vojta, and P. Wölfle, Rev. Mod. Phys. 79, 1015 (2007).

[11] C. Pfleiderer, Rev. Mod. Phys. 81, 1551 (2009).

[12] O. Stockert and F. Steglich, Annu. Rev. Condens. Matter Phys. 2, 79 (2011).

[13] H. v. Löhneysen, T. Pietrus, G. Portisch, H. G. Schlager, A. Schröder, M. Sieck, and T. Trappmann, Phys. Rev. Lett. 72, 3262 (1994).

[14] H. Löhneysen, M. Sieck, O. Stockert, and M. Waffenschmidt, Physica B 223, 471 (1996).

[15] Mathur N. D., Grosche F. M., Julian S. R., Walker I. R., Freye D. M., Haselwimmer R. K. W., and Lonzarich G. G., Nature 394, 39 (1998). 
[16] J. Kondo, Progr. Theoret. Phys. 28, 846 (1962).

[17] J. Kondo, Progr. Theoret. Phys. 32, 37 (1964).

[18] L. Kouwenhoven and L. Glazman, Physics World 14, 33 (2001).

[19] S. Doniach, Physica B 91, 231 (1977).

[20] A. Germann, A. K. Nigam, J. Dutzi, A. Schröder, and H. v. Löhneysen, J. Phys. Colloq. 49, C8 (1988).

[21] A. Germann and H. v Löhneysen, Europhys. Lett. 9, 367 (1989).

[22] H. Löhneysen, A. Schröder, T. Trappmann, and M. Welsch, J. Magn. Magn. Mater. 108, 45 (1992).

[23] L. D. Landau, Sov. Phys. JETP 3 (1957).

[24] L. D. Landau, Sov. Phys. JETP 5 (1957).

[25] L. D. Landau, Sov. Phys. JETP 8 (1959).

[26] I. Y. Pomeranchuk, Zh. Eksp. Teor. Fiz. 35, 524 (1958).

[27] M. Y. Amusia, K. G. Popov, V. R. Shaginyan, and V. A. Stephanovich, Theory of Heavy-Fermion Compounds, Springer Series, 2015.

[28] B. Andraka and G. R. Stewart, Phys. Rev. B 47, 3208 (1993).

[29] K. Umeo, H. Kadomatsu, and T. Takabatake, J. Phys.: Condens. Matter 8, 9743 (1996).

[30] P. Coleman, Handbook of Magnetism and Advanced Magnetic Materials, John Wiley \& Sons, Ltd, 2007.

[31] W. de HaAs, J. De Boer, and G. VAn DËn Berg, Physica (Utrecht) 1, 1115 (1934).

[32] P. W. Anderson, Phys. Rev. 124, 41 (1961).

[33] G. R. Stewart, Z. Fisk, J. O. Willis, and J. L. Smith, Phys. Rev. Lett. 52, 679 (1984).

[34] P. Gegenwart, J. Custers, C. Geibel, K. Neumaier, T. Tayama, K. Tenya, O. Trovarelli, and F. Steglich, Phys. Rev. Lett. 89, 056402 (2002).

[35] P. Coleman, C. Pépin, Q. Si, and R. Ramazashvili, J. Phys.: Condens. Matter 13, R723 (2001).

[36] M. Klein, A. Nuber, F. Reinert, J. Kroha, O. Stockert, and H. V. Löhneysen, Phys. Rev. Lett. 101, 266404 (2008).

[37] R. D. Averitt and A. J. Taylor, J. Phys.: Condens. Matter 14, R1357 (2002). 
[38] J. M. Ziman, Principles of the Theory of Solids, Cambridge University Press, Cambridge, 2 edition, 1972.

[39] A. C. Hewson, The Kondo Problem to Heavy Fermions, Cambridge University Press, 1993.

[40] K. Yamada, Electron Correlation in Metals, Cambridge University Press, 2004.

[41] M. Sigrist, Lecture on Solid State Theory, ETH Zürich, 2011.

[42] J. Kondo, The Physics of Dilute Magnetic Alloys, Cambridge University Press, 2012.

[43] D. R. Hartree, Math. Proc. Cambridge Philos. Soc. 24, 89 (1928).

[44] D. R. Hartree, Math. Proc. Cambridge Philos. Soc. 24, 111 (1928).

[45] A. A. Abrikosov and I. M. Khalatnikov, Rep. Prog. Phys. 22, 329 (1959).

[46] C. L. Seaman, M. B. Maple, B. W. Lee, S. Ghamaty, M. S. Torikachvili, J.-S. Kang, L. Z. Liu, J. W. Allen, and D. L. Cox, Phys. Rev. Lett. 67, 2882 (1991).

[47] B. Andraka and A. M. Tsvelik, Phys. Rev. Lett. 67, 2886 (1991).

[48] C. Pfleiderer, P. Böni, T. Keller, U. K. Rössler, and A. Rosch, Science 316, 1871 (2007).

[49] J. von Delft and H. Schoeller, Ann. Phys. 7, 225 (1998).

[50] J. M. Luttinger, Phys. Rev. 119, 1153 (1960).

[51] F. D. M. Haldane, J. Phys. C 14, 2585 (1981).

[52] A. Imambekov, T. L. Schmidt, and L. I. Glazman, Rev. Mod. Phys. 84, 1253 (2012).

[53] C. Castellani, C. Di Castro, and M. Grilli, Z. Phys. B 103, 137 (1996).

[54] O. Trovarelli, C. Geibel, S. Mederle, C. Langhammer, F. M. Grosche, P. Gegenwart, M. Lang, G. Sparn, and F. Steglich, Phys. Rev. Lett. 85, 626 (2000).

[55] N. Ashcroft and N. Mermin, Festkörperphysik, Oldenbourg, 2007.

[56] P. Phillips, Advanced Solid State Physics, Cambridge University Press, 2012.

[57] M. A. Ruderman and C. Kittel, Phys. Rev. 96, 99 (1954).

[58] K. Yosida, Phys. Rev. 106, 893 (1957).

[59] P. A. M. Dirac, Proc. Roy. Soc. A114, 1 (1927). 
[60] G. Wentzel, Z. Phys. 43, 524 (1927).

[61] E. Fermi, Nuclear Physics, University of Chicago Press, 1950.

[62] M. Born, Z. Phys. 38, 803 (1926).

[63] N. Straumann, Quantenmechanik, Springer Verlag, 2002.

[64] J. Kondo, Theory of Dilute Magnetic Alloys, Academic Press, 1970.

[65] J. Bardeen, L. N. Cooper, and J. R. Schrieffer, Phys. Rev. 108, 1175 (1957).

[66] A. A. Abrikosov, Physics 2, 5 (1965).

[67] O. O. Bernal, D. E. Maclaughlin, A. Amato, R. Feyerherm, F. N. Gygax, A. Schenck, R. H. Heffner, L. P. Le, G. J. Nieuwenhuys, B. Andraka, H. v. Löhneysen, O. Stockert, and H. R. Ott, Phys. Rev. B 54, 13000 (1996).

[68] B. Stroka, A. Schröder, T. Trappmann, H. V. Löhneysen, M. Loewenhaupt, and A. Severing, Z. Phys. B 90, 155 (1993).

[69] B. Cornut and B. Coqblin, Phys. Rev. B 5, 4541 (1972).

[70] K. Yamada, K. Yosida, and K. Hanzawa, Progr. Theoret. Phys. 71, 450 (1984).

[71] J. D. Thompson and Z. Fisk, Phys. Rev. B 31, 389 (1985).

[72] D. Jaccard, H. Wilhelm, K. Alami-Yadri, and E. Vargoz, Physica B 259-261, 1 (1999).

[73] H. Wilhelm and D. Jaccard, Phys. Rev. B 66, 064428 (2002).

[74] A. Demuer, A. T. Holmes, and D. Jaccard, J. Phys.: Condens. Matter 14, L529 (2002).

[75] A. T. Holmes, D. Jaccard, and K. Miyake, Phys. Rev. B 69, 024508 (2004).

[76] M. E. Fisher, Rev. Mod. Phys. 46, 597 (1974).

[77] M. Vojta, Rep. Prog. Phys. 66, 2069 (2003).

[78] J. A. Hertz, Phys. Rev. B 14, 1165 (1976).

[79] A. J. Millis, Phys. Rev. B 48, 7183 (1993).

[80] M. A. Continentino, Phys. Rev. B 47, 11587 (1993).

[81] T. Moriya and T. Takimoto, J. Phys. Soc. Jpn. 64, 960 (1995).

[82] Q. Si, S. Rabello, K. Ingersent, and J. L. Smith, Nature 413, 804 (2001).

[83] T. Senthil, M. Vojta, and S. Sachdev, Phys. Rev. B 69, 035111 (2004). 
[84] I. Paul, C. Pépin, and M. R. Norman, Phys. Rev. Lett. 98, 026402 (2007).

[85] E. Fawcett, Rev. Mod. Phys. 60, 209 (1988).

[86] R. E. Peierls and L. D. Roberts, Physics Today 9, 29 (1956).

[87] J. Sichelschmidt, J. Ferstl, C. Geibel, and F. Steglich, Physica B 359-361, 17 (2005).

[88] A. Schröder, G. Aeppli, R. Coldea, M. Adams, O. Stockert, H. v. Löhneysen, E. Bucher, R. Ramazashvili, and P. Coleman, Nature 407, 351 (2000).

[89] O. Stockert, H. v. Löhneysen, A. Rosch, N. Pyka, and M. Loewenhaupt, Phys. Rev. Lett. 80, 5627 (1998).

[90] A. Amato, D. Jaccard, J. Flouquet, F. Lapierre, J. L. Tholence, R. A. Fisher, S. E. Lacy, J. A. Olsen, and N. E. Phillips, J. Low Temp. Phys. 68, 371 (1987).

[91] H. Tsujil, E. Tanaka, Y. Ode, T. Katoh, T. Mamiya, S. Araki, R. Settai, and Y. Ōnuki, Phys. Rev. Lett. 84, 5407 (2000).

[92] M. Klein, J. Kroha, H. v. Löhneysen, O. Stockert, and F. Reinert, Phys. Rev. B 79, 075111 (2009).

[93] E. Gratz, E. Bauer, H. Nowotny, H. Mueller, S. Zemirli, and B. Barbara, J. Magn. Magn. Mater. 63-64, 312 (1987).

[94] T. Chattopadhyay, H. v. Löhneysen, T. Trappmann, and M. Loewenhaupt, Z. Phys. B 80, 159 (1990).

[95] T. Pietrus, B. Bogenberger, S. Mock, M. Sieck, and H. v. LÖhneysen, Physica B 206-207, 317 (1995).

[96] M. Ruck, G. Portisch, H. G. Schlager, M. Sieck, and H. v. LÖHNEYSEn, Acta Cryst. B 49, 936 (1993).

[97] A. Schröder, J. W. Lynn, R. W. Erwin, M. Loewenhaupt, and H. v. LÖhNEYSEn, Physica B 199-200, 47 (1994).

[98] K. Grube, W. H. Fietz, U. Tutsch, O. Stockert, and H. V. LÖHnEysen, Phys. Rev. B 60, 11947 (1999).

[99] S. Zemirli and B. Barbara, Solid State Commun. 56, 385 (1985).

[100] G. Aeppli, H. Yoshizawa, Y. Endoh, E. Bucher, J. Hufnagl, Y. Onuki, and T. Komatsubara, Phys. Rev. Lett. 57, 122 (1986).

[101] H. G. Schlager, A. Schröder, M. Welsch, and H. v. Löhneysen, J. Low Temp. Phys. 90, 181 (1993).

[102] H. von Löhneysen, A. Neubert, T. Pietrus, A. Schröder, O. Stockert, U. Tutsch, M. Loewenhaupt, A. Rosch, and P. Wölfle, Eur. Phys. J. B 5, 447 (1998). 
[103] P. Gegenwart, J. Custers, Y. Tokiwa, C. Geibel, and F. Steglich, Phys. Rev. Lett. 94, 076402 (2005).

[104] P. Reiss, P. Rourke, G. Zwicknagl, F. M. Grosche, and S. Friedemann, Phys. Status Solidi b 250, 498 (2013).

[105] N. Oeschler, S. Hartmann, A. Pikul, C. Krellner, C. Geibel, and F. Steglich, Physica B 403, 1254 (2008).

[106] J. Custers, P. Gegenwart, C. Geibel, F. Steglich, P. Coleman, and S. Paschen, Phys. Rev. Lett. 104, 186402 (2010).

[107] A. Rosch, A. Schröder, O. Stockert, and H. V. Löhneysen, Phys. Rev. Lett. 79, 159 (1997).

[108] H. V. LÖHNEYSEN, (2017), private communication.

[109] L. Degiorgi, Rev. Mod. Phys. 71, 687 (1999).

[110] D. L. Cox and N. Grewe, Z. Phys. B 71, 321 (1988).

[111] J. KROHA, (2017), private communication.

[112] W.-T. Pong and C. Durkan, J. Phys. D 38, R329 (2005).

[113] V. Madhavan, W. Chen, T. Jamneala, M. F. Crommie, and N. S. WingreEn, Science 280, 567 (1998).

[114] U. FAno, Phys. Rev. 124, 1866 (1961).

[115] S. Ernst, S. Kirchner, C. Krellner, C. Geibel, G. Zwicknagl, F. Steglich, and S. Wirth, Nature 474, 362 (2011).

[116] J. Bork, Y. Zhang, L. Diekhöner, L. Borda, P. Simon, J. Kroha, P. Wahl, and K. Kern, Nat. Phys. 7, 901 (2011).

[117] A. Damascelli, Z. Hussain, and Z.-X. Shen, Rev. Mod. Phys. 75, 473 (2003).

[118] D. Ehm, S. Hüfner, F. Reinert, J. Kroha, P. Wölfle, O. Stockert, C. Geibel, and H. V. Löhneysen, Phys. Rev. B 76, 045117 (2007).

[119] A. M. Awasthi, L. Degiorgi, G. Grüner, Y. Dalichaouch, and M. B. Maple, Phys. Rev. B 48, 10692 (1993).

[120] F. Marabelli, P. Wachter, and J. Franse, J. Magn. Magn. Mater. 62, 287 (1986).

[121] F. Marabelli, P. Wachter, and E. Walker, Solid State Commun. 67, 931 (1988).

[122] L. Degiorgi, Ann. Phys. 15, 571 (2006).

[123] M. Scheffler, T. Weig, M. Dressel, H. Shishido, Y. Mizukami, T. Terashima, T. Shibauchi, and Y. Matsuda, J. Phys. Soc. Jpn. 82, 043712 (2013). 
[124] V. Guritanu, P. Wissgott, T. Weig, H. Winkler, J. Sichelschmidt, M. Scheffler, A. Prokofiev, S. Kimura, T. Iizuka, A. M. Strydom, M. Dressel, F. Steglich, K. Held, and S. Paschen, Phys. Rev. B 87, 115129 (2013).

[125] A. LoidL, (2017), private communication.

[126] D. N. Basov, R. D. Averitt, D. van Der Marel, M. Dressel, and K. Haule, Rev. Mod. Phys. 83, 471 (2011).

[127] D. L. Maslov and A. V. Chubukov, Rep. Prog. Phys. 80, 026503 (2017).

[128] G. Bossé, L. S. Bilbro, R. V. Aguilar, L. Pan, W. Liu, A. V. Stier, Y. Li, L. H. Greene, J. Eckstein, and N. P. Armitage, Phys. Rev. B 85, 155105 (2012).

[129] G. Bossé, L. Pan, Y. S. Li, L. H. Greene, J. Eckstein, and N. P. Armitage, Phys. Rev. B 93, 085104 (2016).

[130] N. J. Laurita, C. M. Morris, S. M. Koohpayeh, P. F. S. Rosa, W. A. Phelan, Z. Fisk, T. M. McQueen, and N. P. Armitage, Phys. Rev. B 94, 165154 (2016).

[131] S. L. Dexheimer, Terahertz Spectroscopy: Principles and Applications, Taylor \& Francis Group, 2008.

[132] Y. S. LEE, Principles of Terahertz Science and Technology, Springer Verlag, 2009.

[133] M. Perenzoni and D. J. PAul, Physics and Applications of Terahertz Radiation, Springer Verlag, 2014.

[134] D. Fausti, R. I. Tobey, N. Dean, S. Kaiser, A. Dienst, M. C. Hoffmann, S. Pyon, T. Takayama, H. Takagi, and A. Cavalleri, Science 331, 189 (2011).

[135] R. Matsunaga and R. Shimano, Phys. Rev. Lett. 109, 187002 (2012).

[136] R. Matsunaga, Y. I. Hamada, K. Makise, Y. Uzawa, H. Terai, Z. Wang, and R. Shimano, Phys. Rev. Lett. 111, 057002 (2013).

[137] T. Kampfrath, K. Tanaka, and K. A. Nelson, Nat. Photon. 7, 680 (2013).

[138] R. Matsunaga, N. Tsuji, H. Fujita, A. Sugioka, K. Makise, Y. Uzawa, H. Terai, Z. Wang, H. Aoki, and R. Shimano, Science 345, 1145 (2014).

[139] M. Mitrano, A. Cantaluppi, D. Nicoletti, S. Kaiser, A. Perucchi, S. Lupi, P. D. Pietro, D. Pontiroli, M. Ricco, S. R. Clark, D. JAKSCH, and A. CAVAlleri, Nature 530, 461 (2016).

[140] A. Kirilyuk, A. V. Kimel, and T. Rasing, Rev. Mod. Phys. 82, 2731 (2010). 
[141] J. Faist, F. Capasso, D. L. Sivco, C. Sirtori, A. L. Hutchinson, and A. Y. CHo, Science 264, 553 (1994).

[142] S. Ganichev and W. PRetTl, Intense Terahertz Excitation of Semiconductors, Oxford University Press, 2006.

[143] M. Schall, M. Walther, and P. Uhd Jepsen, Phys. Rev. B 64, 094301 (2001).

[144] R. Boyd, Nonlinear Optics, Academic Press, Inc., 2008.

[145] K. Kawase, M. Sato, T. Taniuchi, and H. Ito, Appl. Phys. Lett. 68, 2483 (1996).

[146] H. Hirori, A. Doi, F. Blanchard, and K. Tanaka, Appl. Phys. Lett. 98, 091106 (2011).

[147] P. C. M. Planken, H.-K. Nienhuys, H. J. Bakker, and T. Wenckebach, J. Opt. Soc. Am. B 18, 313 (2001).

[148] F. Blanchard, L. Razzari, H.-C. Bandulet, G. Sharma, R. Morandotti, J.-C. Kieffer, T. Ozaki, M. Reid, H. F. Tiedje, H. K. Haugen, and F. A. Hegmann, Opt. Express 15, 13212 (2007).

[149] F. Pan, G. Knöpfle, C. Bosshard, S. Follonier, R. Spreiter, M. S. Wong, and P. Günter, Appl. Phys. Lett. 69, 13 (1996).

[150] M. Stillhart, A. Schneider, and P. Günter, J. Opt. Soc. Am. B 25, 1914 (2008).

[151] F. D. J. Brunner, O.-P. Kwon, S.-J. Kwon, M. JazbinšEK, A. SchneIder, and P. GÜNTER, Opt. Express 16, 16496 (2008).

[152] C. HiRlimann, Further Methods for the Generation of Ultrashort Optical Pulses, pp. 89-123, Springer New York, 2005.

[153] A. Schneider, M. Neis, M. Stillhart, B. Ruiz, R. U. A. Khan, and P. Günter, J. Opt. Soc. Am. B 23, 1822 (2006).

[154] F. Pockels, Math. Phys. Kl. 39, 1 (1893).

[155] R. W. LEE, Appl. Opt. 8, 1385 (1969).

[156] V. Lucarini, K. E. Peiponen, J. J. SaArinen, and E. M. Vartiainen, Kramers-Kronig Relations in Optical Materials Research, Springer Verlag, 2005.

[157] M. Dressel and M. Scheffler, Ann. Phys. 15, 535 (2006).

[158] M. Scheffler, M. Dressel, M. Jourdan, and H. Adrian, Nature 438, 1135 (2005).

[159] P. Gegenwart, (2017), private communication.

[160] J. M. Whittaker, Proc. Edinb. Math. Soc. 1, 169 (1928). 
[161] M. Unser, Proc. IEEE 88, 569 (2000).

[162] M. Werner, Digitale Signalverarbeitung mit MATLAB, Springer Verlag, 2006.

[163] T. Löffler, T. Hahn, M. Thomson, F. Jacob, and H. G. Roskos, Opt. Express 13, 5353 (2005).

[164] J. D. Jackson, Klassische Elektrodynamik, Walter de Gruyter, 2006.

[165] A. Sell, A. Leitenstorfer, and R. Huber, Opt. Lett. 33, 2767 (2008).

[166] S.-W. Huang, E. Granados, W. R. Huang, K.-H. Hong, L. E. Zapata, and F. X. Kärtner, Opt. Lett. 38, 796 (2013).

[167] W. R. Huang, S.-W. Huang, E. Granados, K. Ravi, K.-H. Hong, L. E. Zapata, and F. X. Kärtner, J. Mod. Opt. 62, 1486 (2015).

[168] M. Shalaby, C. Vicario, and C. P. Hauri, Intense THz source based on BNA organic crystal pumped at conventional Ti:Sapphire wavelength, in $201641 s t$ International Conference on Infrared, Millimeter, and Terahertz waves (IRMMW-THz), pp. 1-1, 2016.

[169] X. C. Zhang, A. Shkurinov, and Y. Zhang, Nat. Photon. 11, 16 (2017).

[170] T. Kampfrath, A. Sell, G. Klatt, A. Pashkin, S. Mährlein, T. Dekorsy, M. Wolf, M. Fiebig, A. Leitenstorfer, and R. Huber, Nat. Photon. 5, 31 (2011).

[171] M. Schultze, E. M. Bothschafter, A. Sommer, S. Holzner, W. Schweinberger, M. Fiess, M. Hofstetter, R. Kienberger, V. Apalkov, V. S. Yakovlev, M. I. Stockman, and F. Krausz, Nature 493, 75 (2013).

[172] O. Schubert, M. Hohenleutner, F. Langer, B. Urbanek, C. Lange, U. Huttner, D. Golde, T. Meier, M. Kira, S. W. Koch, and R. Huber, Nat. Phys. 8, 119 (2014).

[173] J. Corson, J. Orenstein, S. Oh, J. O'Donnell, and J. N. Eckstein, Phys. Rev. Lett. 85, 2569 (2000).

[174] T. Matsuoka, T. Fujimoto, K. Tanaka, S. Miyasaka, S. Tajima, K. Fujir, M. Suzuki, and M. Tonouchi, Physica C 469, 982 (2009).

[175] J. Zhang, J. Yong, I. Takeuchi, R. L. Greene, and R. D. Averitt, ArXiv e-prints (2015).

[176] E. Beaurepaire, G. M. Turner, S. M. Harrel, M. C. Beard, J.-Y. Bigot, and C. A. Schmuttenmaer, Appl. Phys. Lett. 84, 3465 (2004).

[177] N. Kida, H. Murakami, and M. Tonouchi, Terahertz Optics in Strongly Correlated Electron Systems, pp. 271-330, Springer Verlag, 2005.

[178] J. Orenstein, J. Corson, S. Oh, and J. N. Eckstein, Ann. Phys. 15, 596 (2006). 
[179] K. Takahashi, N. Kida, and M. Tonouchi, Phys. Rev. Lett. 96, 117402 (2006).

[180] J. van Tilborg, C. B. Schroeder, C. V. Filip, C. Tóth, C. G. R. Geddes, G. Fubiani, R. Huber, R. A. Kaindl, E. Esarey, and W. P. Leemans, Phys. Rev. Lett. 96, 014801 (2006).

[181] A. Agrawal and A. Nahata, Opt. Express 14, 1973 (2006).

[182] Y. Zhao and D. Grischkowsky, Opt. Lett. 31, 1534 (2006).

[183] W. J. Padilla, A. J. Taylor, C. Highstrete, M. Lee, and R. D. Averitt, Phys. Rev. Lett. 96, 107401 (2006).

[184] M. Liu, H. Hwang, H. Tao, A. Strikwerda, K. Fan, G. Keiser, A. Sternbach, K. West, S. Kittiwatanakul, J. Lu, S. Wolf, F. Omenetto, X. Zhang, K. Nelson, and R. Averitt, Nature 487, 345 (2012).

[185] R. Valdés Aguilar, A. V. Stier, W. Liu, L. S. Bilbro, D. K. George, N. Bansal, L. Wu, J. Cerne, A. G. Markelz, S. Oh, and N. P. Armitage, Phys. Rev. Lett. 108, 087403 (2012).

[186] P. R. Bowlan, J. Bowlan, S. Trugman, R. V. Aguilar, J. Qi, X. Liu, J. Furdyna, A. Taylor, D. Yarotski, and R. Prasankumar, Nonlinear phonon dynamics in the topological insulator Bi2Se3 driven by intense $\mathrm{THz}$ pulses, in International Conference on Ultrafast Phenomena, p. UTu3A.2, Optical Society of America, 2016.

[187] K. Makino, S. Kuromiya, K. Takano, K. Kato, M. Nakajima, H. Iida, M. Kinoshita, Y. Saito, J. Tominaga, and T. Nakano, Multi-layered topological insulator for $\mathrm{THz}$ detection, in Advanced Photonics 2016, p. SeW1E.3, Optical Society of America, 2016.

[188] B. P. Gorshunov, A. S. Prokhorov, I. E. Spektor, A. A. Volkov, M. Dressel, M. Dumm, and T. Matsumura, J. Exp. Theor. Phys. 101, 913 (2005).

[189] M. Z. Hasan, Y.-D. Chuang, D. Qian, Y. W. Li, Y. Kong, A. Kuprin, A. V. Fedorov, R. Kimmerling, E. Rotenberg, K. Rossnagel, Z. Hussain, H. Koh, N. S. Rogado, M. L. Foo, and R. J. Cava, Phys. Rev. Lett. 92, 246402 (2004).

[190] A. Bostwick, T. Ohta, T. Seyller, K. Horn, and E. Rotenberg, Nat. Phys. 3, 36 (2007).

[191] E. Knoesel, A. Hotzel, and M. Wolf, Phys. Rev. B 57, 12812 (1998).

[192] W. Metzner and D. Vollhardt, Phys. Rev. Lett. 62, 324 (1989).

[193] G. Kotliar, S. Y. Savrasov, K. Haule, V. S. Oudovenko, O. Parcollet, and C. A. Marianetti, Rev. Mod. Phys. 78, 865 (2006). 
[194] S. Burdin, A. Georges, and D. R. Grempel, Phys. Rev. Lett. 85, 1048 (2000).

[195] K. Haule and G. Kotliar, Nat. Phys. 5, 796 (2009).

[196] A. Georges, G. Kotliar, W. Krauth, and M. J. Rozenberg, Rev. Mod. Phys. 68, 13 (1996).

[197] F. Marabelli and P. Wachter, Phys. Rev. B 42, 3307 (1990).

[198] A. Sumiyama, Y. Oda, H. Nagano, Y. Ånuki, K. Shibutani, and T. Komatsubara, J. Phys. Soc. Jpn. 55, 1294 (1986).

[199] F. F. Assaad, Phys. Rev. B 65, 115104 (2002).

[200] M. Fleischhauer, A. Imamoglu, and J. P. Marangos, Rev. Mod. Phys. $77,633(2005)$.

[201] H. E. Türeci, M. Hanl, M. Claassen, A. Weichselbaum, T. Hecht, B. Braunecker, A. Govorov, L. Glazman, A. Imamoglu, and J. von Delft, Phys. Rev. Lett. 106, 107402 (2011).

[202] S. Smolka, W. Wuester, F. Haupt, S. Faelt, W. Wegscheider, and A. Imamoglu, Science 346, 332 (2014).

[203] G. Nieva, E. Osquiguil, J. Guimpel, M. Maenhoudt, B. Wuyts, Y. Bruynseraede, M. B. Maple, and I. K. Schuller, Appl. Phys. Lett. 60, 2159 (1992).

[204] R. D. Averitt, G. Rodriguez, A. I. Lobad, J. L. W. Siders, S. A. Trugman, and A. J. Taylor, Phys. Rev. B 63, 140502 (2001).

[205] L. Perfetti, P. A. Loukakos, M. Lisowski, U. Bovensiepen, H. Eisaki, and M. Wolf, Phys. Rev. Lett. 99, 197001 (2007).

[206] M. Fiebig, J. Phys. D 38, R123 (2005).

[207] N. A. Spaldin and M. Fiebig, Science 309, 391 (2005).

[208] R. C. Jones, J. Opt. Soc. Am. 31, 488 (1941).

[209] C. Rullière, T. Amand, and X. Marie, Spectroscopic Methods for Analysis of Sample Dynamics, pp. 223-281, Springer New York, 2005.

[210] R. T. Hall and J. M. Dowling, J. Chem. Phys. 47, 2454 (1967).

[211] M. van Exter, C. Fattinger, and D. Grischkowsky, Opt. Lett. 14, 20 (1989).

[212] W. Lauterborn and T. Kurz, Coherent Optics: Fundamentals and Applications, Springer Science \& Business Media, 2003.

[213] M. Naftaly and R. Miles, Opt. Commun. 280, 291 (2007).

[214] G. R. Stewart, Rev. Mod. Phys. 56, 755 (1984). 
[215] B. Gorshunov, N. Sluchanko, A. Volkov, M. Dressel, G. Knebel, A. LoidL, and S. KuniI, Phys. Rev. B 59, 1808 (1999).

[216] E. J. Singley, D. N. Basov, E. D. Bauer, and M. B. Maple, Phys. Rev. B 65, 161101 (2002).

[217] M. Dressel, N. Kasper, K. Petukhov, D. N. Peligrad, B. Gorshunov, M. Jourdan, M. Huth, and H. Adrian, Phys. Rev. B 66, 035110 (2002).

[218] N. S. Vidhyadhiraja and D. E. Logan, J. Phys.: Condens. Matter 17, 2959 (2005). 


\section{A. $\mathrm{THz}$ generation}

\section{A.1. Analytical calculation of a $\mathrm{THz}$ pulse generated by optical rectification}

The generated T-ray pulse from an optical rectification process can be analytically calculated [153]. A detailed explanation of the optical rectification is presented in section 5.4.1. Here, for a simplified case where the absorption is neglected, the emitted $\mathrm{THz}$ pulse is calculated. Furthermore, a constant refractive index $n_{0}$ in the range of the pump spectrum is assumed. The generated electric field of the $\mathrm{THz}$ pulse depends on the propagation distance $z$ through the generation crystal as well as the refractive index $n$ in the $\mathrm{THz}$ range [153]:

$$
E_{\mathrm{THz}}(\omega, z)=\frac{\mu_{0} \chi^{(2)} \omega I_{0}(\omega)}{i \cdot n_{0}\left[n+n_{\mathrm{g}}\right]} \cdot \frac{e^{-i \frac{\omega \cdot n}{c} z}-e^{-i \frac{\omega \cdot n_{\mathrm{g}}}{c} z}}{i \frac{\omega}{c}\left[n-n_{\mathrm{g}}\right]}
$$

$I_{0}(\omega)$ is the spectral intensity of the pump laser, which is centered at $\omega_{0}$, and $n_{\mathrm{g}}$ expresses the optical group refractive index of the NIR pump beam. Eq. A.1 depicts a linear dependence on $\chi^{(2)}$ of the emitted $\mathrm{THz}$ field, the pump laser intensity as well as the photon energy $\hbar \omega$. The second term in Eq. A.1 is maximized if the optical group refractive index $n_{\mathrm{g}}$ of the pump beam matches with the refractive index $n$, which sets the restrictive condition called phase matching (or velocity matching)

$$
n_{\mathrm{g}} \approx n
$$

In a not perfectly phase-matched crystal, the destructive interference of the T-rays generated in different crystal layers sets in after a critical thickness $z_{\text {crit }}$. The $z_{\text {crit }}$ is given by the beating frequency described in the numerator of the second term as well as the dispersion of the generation crystal ${ }^{2}$

$$
z_{\text {crit }}=\frac{2 \pi}{\frac{\omega}{2 c}\left[n-n_{\mathrm{g}}\right]}
$$

\footnotetext{
${ }^{1} n_{\mathrm{g}}\left(\omega_{0}\right)=n\left(\omega_{0}\right)+\left.\omega_{0} \frac{\partial n}{\partial \omega}\right|_{\omega=\omega_{0}}$

${ }^{2}$ The dispersion of the generation crystal is captured by the term $n-n_{\mathrm{g}}$.
} 
In the limit of perfect phase matching and a dispersion-free generation crystal, the critical thickness $z_{\text {crit }}$ diverges.

\section{A.2. Experimental: optical-rectification process in $\mathrm{ZnTe}(110)$}

Only non-centrosymmetric structure can have a second-order susceptibility term, which is further highly sensitive to the single crystal orientation. This fact makes second-order processes a powerful characterization tool, which allows for deep insights into crystalline materials [206, 207]. Here, the $\mathrm{THz}$ generation process in $\mathrm{ZnTe}(110)$ is experimentally confirming the properties described in section 5.4 .2

Anisotropy measurement. A second-order coherent optical response of a non-centrosymmetric crystals is generally anisotropic. The anisotropy of the generation crystal is measured by rotating it, while the incident light polarization as well as the polarization of the detected $\chi^{(2)}$ response (here T-rays) is kept fixed. There are two commonly used configurations, in which anisotropy measurements are performed: T-rays and the NIR pump beam polarization are parallel ( $\mathrm{p}-\mathrm{p}$ configuration) or perpendicular ( $\mathrm{p}$-s configuration) with respect to each other.

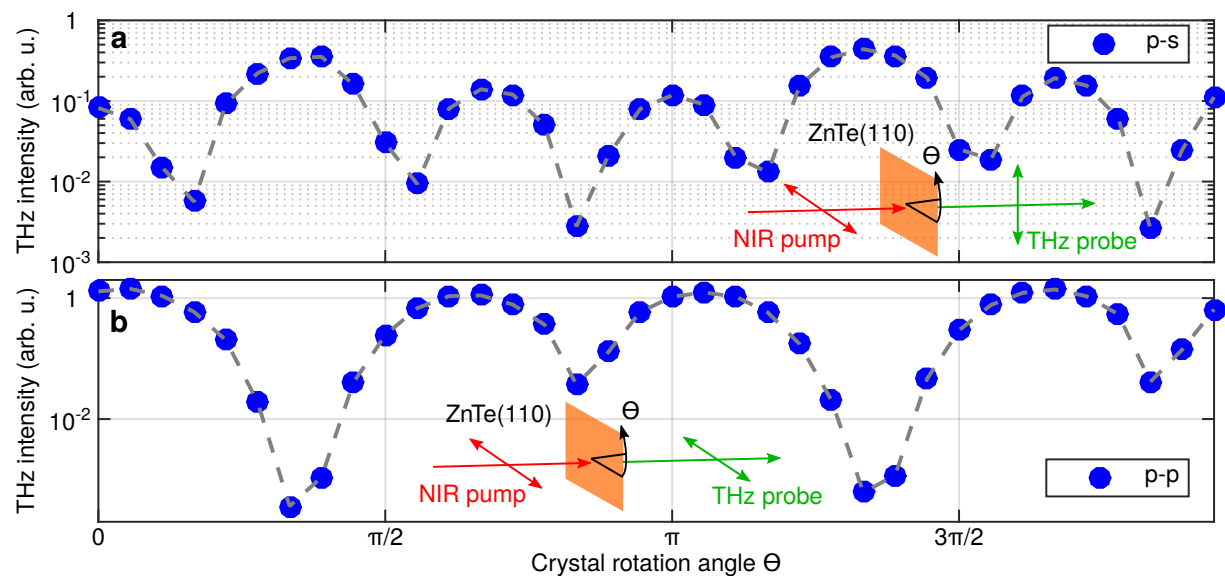

Figure A.1.: Anisotropy measurements of a $0.5 \mathrm{~mm}$ thin $\mathrm{ZnTe}(110)$ crystal under normal incidence. The emitted $\mathrm{THz}$ intensity is monitored as function of the relative rotation angle $\Theta$, as shown in the insets. (a) shows the emitted $\mathrm{THz}$ intensity as function of the rotation angle, while the NIR pump beam is s-polarized and the p-polarized $\mathrm{THz}$ radiation is detected (p-s configuration). (b) shows the anisotropy measurement, where the incident NIR pump beam as well as the detected $\mathrm{THz}$ radiation are p-polarized ( $\mathrm{p}-\mathrm{p}$ configuration). The experimental findings are in excellent agreement with the expected behavior, see Fig. 5.5 
Note that the maximum T-ray intensities for the p-s and the p-p configuration do not occur for the same crystal orientation, see also Fig. 5.5. At the maximum intensity in the p-p configuration, the emitted T-rays are parallel polarized to the NIR pump beam. However, the $\mathrm{THz}$ radiation emitted at the p-s peak is perpendicularly polarized to the pump beam polarization. Since the p-p peak is higher, as expected in Fig. 5.5. the author operates the setup in the most efficient p-p configuration. This experimentally confirms the discussion in section 5.4 .2 . 



\section{B. $\mathrm{THz}$ detection}

\section{B.1. Analytical description of the electro-optical sampling}

With a temporally resolved detection scheme, the electric field is directly measured as function of time, which gives access to the phase information of the $\mathrm{THz}$ pulse. The mathematical description is evocative of the generation process, see also section 5.4 .2 . Therefore, the author used a ZnTe single crysta ${ }^{1}$ also as the detection crystal to do the electro-optical sampling. The electro-optical three-rank tensor is projected onto a 3-by-6 matrix $r^{\mathrm{EO}}$, where for the space group symmetry of ZnTe only three entries are allowed and have to be equal ${ }^{2} r_{14}=r_{25}=r_{36}$. The induced polarization $\vec{P}$ by the electro-optical sampling process is described by the following equation:

$$
\left(\begin{array}{c}
P_{\mathrm{x}} \\
P_{\mathrm{y}} \\
P_{\mathrm{z}}
\end{array}\right):=4 \epsilon_{0} r_{14}\left(\begin{array}{cccccc}
0 & 0 & 0 & 1 & 0 & 0 \\
0 & 0 & 0 & 0 & 1 & 0 \\
0 & 0 & 0 & 0 & 0 & 1
\end{array}\right)\left(\begin{array}{c}
E_{\mathrm{x}} E_{\mathrm{x}}^{\mathrm{THz}} \\
E_{\mathrm{y}} E_{\mathrm{y}}^{\mathrm{THz}} \\
E_{\mathrm{z}} E_{\mathrm{z}}^{\mathrm{THz}} \\
E_{\mathrm{y}} E_{\mathrm{z}}^{\mathrm{THz}}+E_{\mathrm{z}} E_{\mathrm{y}}^{\mathrm{THz}} \\
E_{\mathrm{z}} E_{\mathrm{x}}^{\mathrm{THz}}+E_{\mathrm{x}} E_{\mathrm{z}}^{\mathrm{THz}} \\
E_{\mathrm{x}} E_{\mathrm{y}}^{\mathrm{THz}}+E_{\mathrm{y}} E_{\mathrm{x}}^{\mathrm{THz}}
\end{array}\right)
$$

where $E_{i}$ is the $i$-th component of the probe beam and $E_{i}^{\mathrm{THz}}$ the $i$-th component of the $\mathrm{THz}$ electric field. The crystal properties are best described in spherical coordinates, where $\phi$ is the polar and $\Theta$ the azimuthal angle:

$$
\vec{E}^{\mathrm{THz}}=\left(\begin{array}{c}
\cos \theta^{\mathrm{THz}} \cdot \cos \phi^{\mathrm{THz}} \\
\cos \theta^{\mathrm{THz}} \cdot \sin \phi^{\mathrm{THz}} \\
\sin \theta^{\mathrm{THz}}
\end{array}\right) \quad \vec{E}=\left(\begin{array}{c}
\cos \theta \cdot \cos \phi \\
\cos \theta \cdot \sin \phi \\
\sin \theta
\end{array}\right)
$$

Hence, the THz-induced polarization $\vec{P}$ for the ZnTe crystal reads as follows:

\footnotetext{
${ }^{1}$ The point group symmetry of ZnTe is $\overline{4} 3 \mathrm{~m}$.

${ }^{2} r_{14}=d_{41} \approx 3.9 \frac{\mathrm{pm}}{\mathrm{V}}$, for ZnTe single crystals [147].
} 


$$
\begin{aligned}
\left(\begin{array}{c}
P_{\mathrm{x}} \\
P_{\mathrm{y}} \\
P_{\mathrm{z}}
\end{array}\right)= & 4 \epsilon_{0} r_{14} E_{0} E_{0}^{\mathrm{THz}} \\
& \cdot\left(\begin{array}{c}
\cos \theta \sin \phi \sin \theta^{\mathrm{THz}}+\sin \theta \cos \theta^{\mathrm{THz}} \sin \phi^{\mathrm{THz}} \\
\sin \theta \cos \theta^{\mathrm{THz}} \cos \phi^{\mathrm{THz}}+\cos \theta \cos \phi \sin \theta^{\mathrm{THz}} \\
\cos \theta \cos \phi \cos \theta^{\mathrm{THz}} \sin \phi^{\mathrm{THz}}+\cos \theta \sin \phi \cos \theta^{\mathrm{THz}} \cos \phi^{\mathrm{THz}}
\end{array}\right)
\end{aligned}
$$

Since the electro-optical sampling tensor $r^{\mathrm{EO}}$ is equivalent to the second-order susceptibility tensor $d$ describing the optical rectification process, ZnTe(110)-cut crystals are also desirable detection crystals, see Fig. 5.5. Hence, the polar angles $\phi$ and $\phi^{\mathrm{THz}}$ are fixed to $\frac{\pi}{4}$. In contrast to the generation process, there are now two independent beams involved in the electro-optical detection process: The T-ray pulse as well as the NIR probe beam. In order to have a high sensitivity, the $\mathrm{THz}$ pulse should have a maximum effect on the ellipticity of the probe pulse polarization. Hence, the quadratically induced polarization $\vec{P}$ should be orthogonal to the incident NIR probe pulse polarization $\vec{E}$, causing the optical probe to evolve into an elliptically polarized beam. Hence, a maximum effect on the ellipticity is reached if the following equation is fulfilled:

$$
0=\vec{P} \cdot \vec{E}
$$

where the THz-induced polarization $\vec{P}$ is a function of the NIR-probe polarization as well as the THz-pulse polarization. Fig. B.1 shows the scalar product as function of the azimuthal angles $\Theta$ (radial coordinate) and $\theta^{\mathrm{THz}}$ (polar coordinate), while the polar angles $\phi$ and $\phi^{\mathrm{THz}}$ are fixed to $\frac{\pi}{4}$ (by the crystal cut).

Fig. B.1 illustrates all solutions of Eq. B.4 as the scalar product between $\vec{E}$ and $\vec{P}$ is plotted as function of the $\mathrm{THz}$ polarization and the NIR probe beam polarization. 


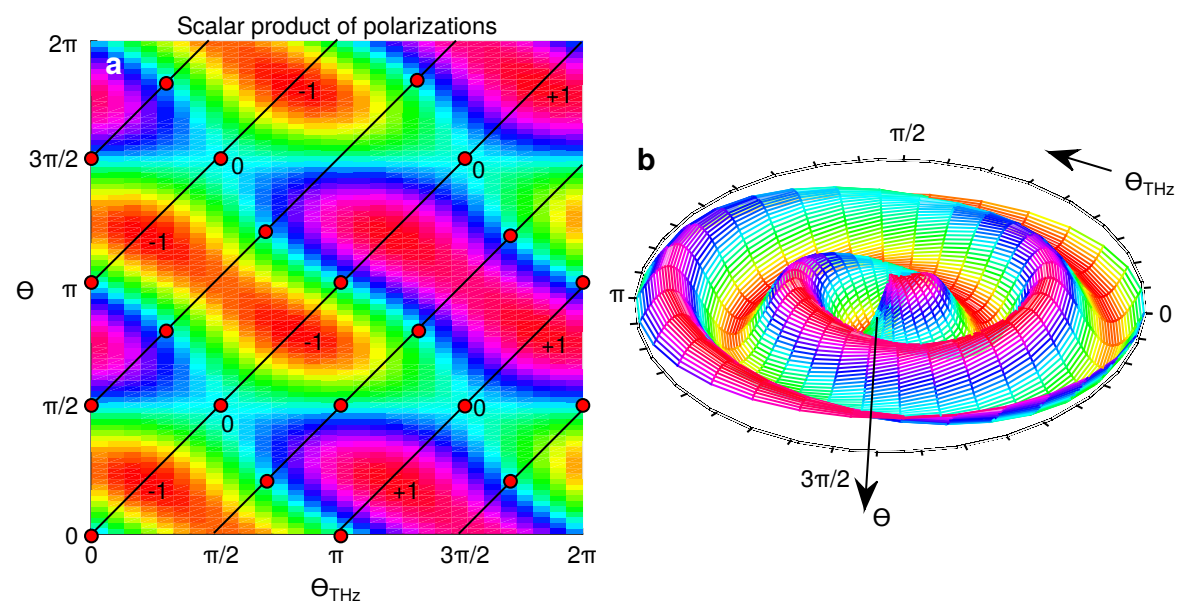

Figure B.1.: (a,b) Illustration of the scalar product between the THz-induced polarization and NIR probe beam polarization. In order to have maximum sensitivity, the induced polarization has to be perpendicular to the probe beam polarization (turquoise color in the plot corresponds to $\vec{P} \cdot \vec{E}=0$ ). The red dots indicate the experimentally relevant solutions, as preferably only s- and p-polarized light is used.

\section{B.2. Analytical description of birefringence measurement}

The detection scheme used to measure the birefringence of the electro-optical sampling crystal is best described using the Jones calculus [208, 209]. It describes the polarization behavior of radiation as it passes optical elements.

In the free-space electro-optical sampling scheme, see also Fig. 5.7, the quarter-wave plate is rotated by $\pi / 4$ with respect to the incident probe beam polarization:

$$
\begin{aligned}
M_{\lambda / 4} & =\left(\begin{array}{cc}
\cos \phi & -\sin \phi \\
\sin \phi & \cos \phi
\end{array}\right) \cdot e^{-i \frac{\pi}{4}}\left(\begin{array}{ll}
1 & 0 \\
0 & i
\end{array}\right) \cdot\left(\begin{array}{cc}
\cos \phi & \sin \phi \\
-\sin \phi & \cos \phi
\end{array}\right) \\
& =\frac{1}{\sqrt{2}}\left(\begin{array}{cc}
1 & -i \\
-i & 1
\end{array}\right) .
\end{aligned}
$$


Hence, the Jones vector of the light reaching the balanced photodiode reads as:

$$
\begin{aligned}
\vec{J} & =\frac{1}{\sqrt{2}}\left(\begin{array}{cc}
1 & -i \\
-i & 1
\end{array}\right) \frac{1}{\sqrt{1+\Delta \phi^{2}}}\left(\begin{array}{cc}
0 & i \Delta \phi \\
0 & 1
\end{array}\right)\left(\begin{array}{l}
0 \\
1
\end{array}\right) \\
& =\frac{1}{\sqrt{2\left(1+\Delta \phi^{2}\right)}}\left(\begin{array}{c}
i(\Delta \phi-1) \\
1+\Delta \phi
\end{array}\right),
\end{aligned}
$$

where $\Delta \phi$ is the differential phase retardation. The differential phase retardation is given by the following equation [144]:

$$
\Delta \phi=\Delta n \frac{|\vec{k}| D}{2 \pi}=\frac{|\vec{k}|}{2 \pi} D n_{O}^{3} r_{41} E^{\mathrm{THz}}
$$

where $|\vec{k}|$ is the wave vector, $D$ the thickness of the detection crystal, $n_{O}$ the refractive index and $r_{41}$ the nonlinear coefficient. Hence, the maximum differential phase retardation $\Delta \phi$ between the two optical axes can estimated to be in the range of $\sim 6 \%$, using the following characteristic numbers of the author's setup:

\begin{tabular}{c|l} 
quantity & value \\
\hline \hline$|\vec{k}|=\frac{\nu}{c}$ & $\frac{10^{15} \mathrm{~Hz}}{3 \cdot 1^{8} \mathrm{~m} / \mathrm{s}} \approx 3 \cdot 10^{6} \frac{1}{\mathrm{~m}}$ \\
\hline$D$ & $5 \cdot 10^{-4} \mathrm{~m}$ \\
\hline$n_{O}$ & $2.8[147]$ \\
\hline$r_{41}$ & $3.9 \mathrm{pm} / \mathrm{V}[147]$ \\
\hline$E_{\max }^{\mathrm{THz}}$ & $\sim 5 \mathrm{kV} / \mathrm{cm}$, see section 5.10
\end{tabular}

Table B.1: Characteristic numbers of the THz-TDS setup built and developed by the author for the Kondo-lattice experiments.

The signal acquired by the balanced photodiode is the difference between two perpendicularly polarized intensities, as a Wollaston prism is used to spatially split the incident light $\vec{J}$ into an $\mathrm{x}$ - and $\mathrm{y}$ - component:

$$
\begin{aligned}
J_{\mathrm{x}} J_{\mathrm{x}}^{\dagger}-J_{\mathrm{y}} J_{\mathrm{y}}^{\dagger} & =\frac{1}{2\left(1+\Delta \phi^{2}\right)}\left[(\Delta \phi-1)^{2}-(1+\Delta \phi)^{2}\right] \\
& =\frac{-2 \Delta \phi}{1+\Delta \phi^{2}} \approx-2 \Delta \phi \propto E^{\mathrm{THz}} .
\end{aligned}
$$

If $\mathrm{THz}$ electric field is zero, the signal acquired on the balanced photodiode is also zero. However, if $E^{\mathrm{THz}} \neq 0$ the balanced photodiode measures a finite value. Note that this detection scheme is phase-sensitive. 


\section{Water absorption}

\section{C.1. Molecular modes of $\mathrm{H}_{2} \mathrm{O}$}

The water molecule $\mathrm{H}_{2} \mathrm{O}$ shows many absorption lines in the lower $\mathrm{THz}$ frequencies. The humidity in air is already sufficient to show strong and sharp water absorption lines. Therefore, the system has to be purged with dry air $\left(\mathrm{N}_{2}\right)$ to avoid them. Fig. C.1 illustrates the effects of water on the $\mathrm{THz}$ pulse in the time domain.

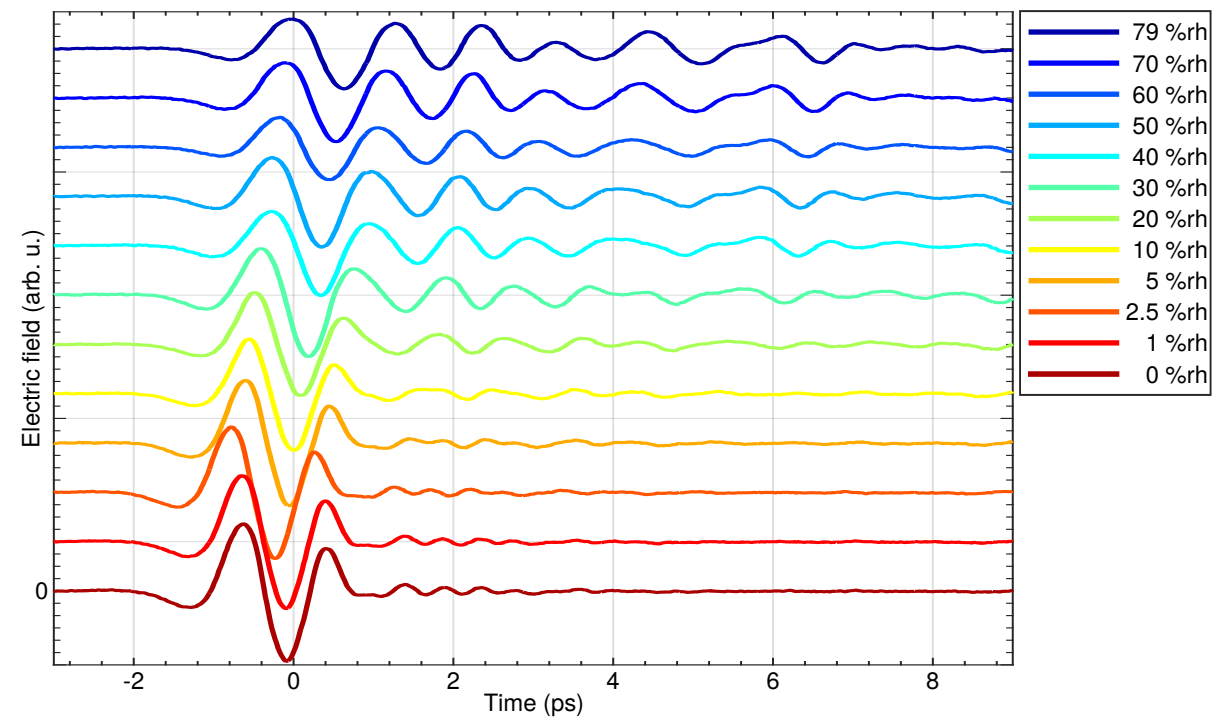

Figure C.1.: Different $\mathrm{THz}$ time traces collected for different relative humidities. In the high-humidity case (blue), many post-pulse oscillations are observed. These oscillations can be damped by purging the setup with dry air (red).

The distinct humidity changes in the time domain lead to humidity-dependent spectra. 1 as shown in Fig. C.2.

\footnotetext{
${ }^{1}$ Note that in THz-TDS the frequency resolution is nowhere near as good as in THz-FDS [156]. However, in contrast to THz-FDS, one single time trace contains the spectral information over an entire decade of frequencies. Furthermore the time scale is directly accessible.
} 


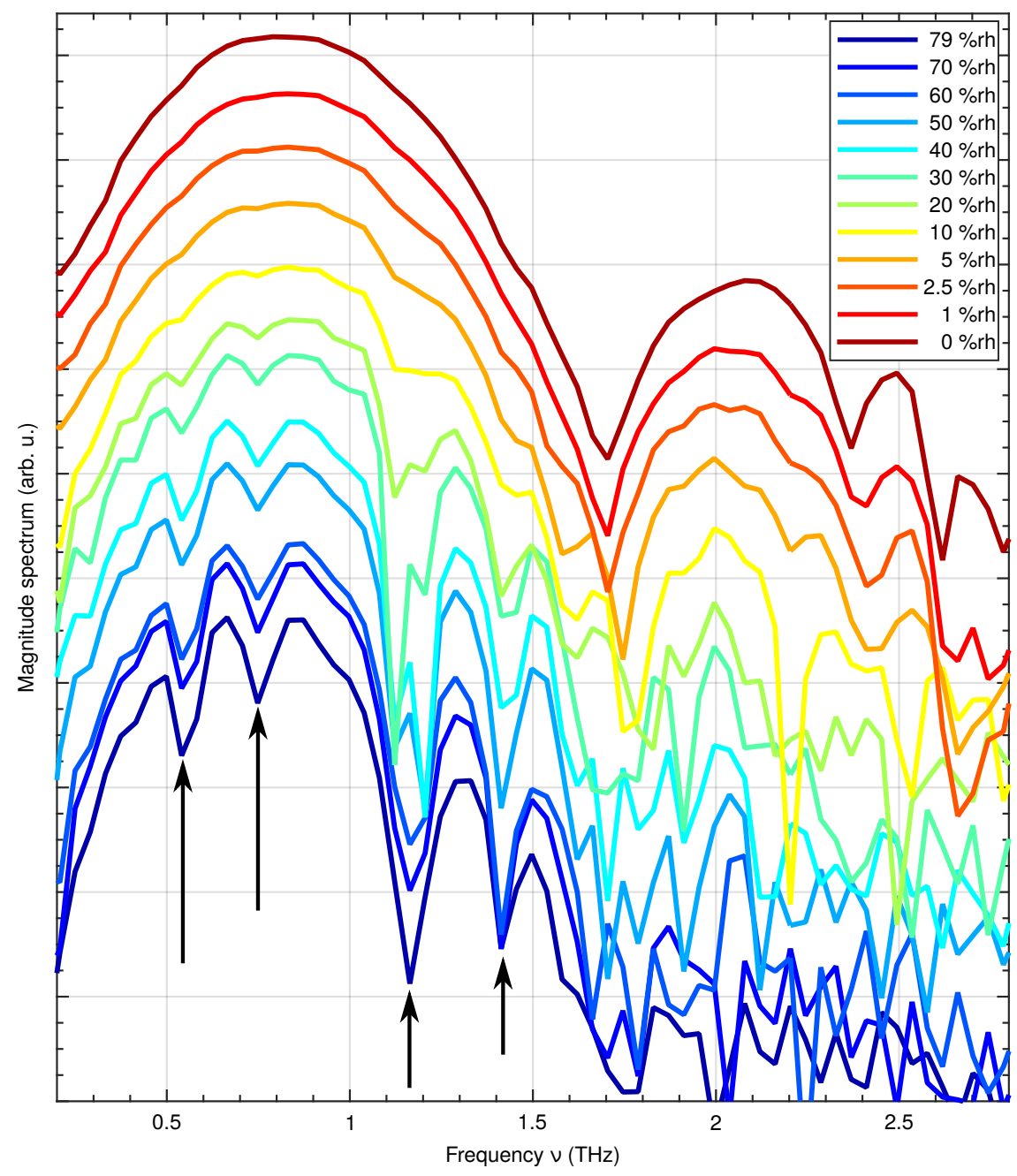

Figure C.2.: Magnitude of Fourier transform for different relative humidity values. It is apparent that the resonances at $0.54,0.74,1.16$ and $1.41 \mathrm{THz}$ vanish by purging the system with dry air (indicated by black arrows). The Fourier transform was conducted for $12 \mathrm{ps}$ long time traces, leading to a spectral resolution of $\Delta \nu=84 \mathrm{GHz}$. The gained $\mathrm{H}_{2} \mathrm{O}$ resonances coincide with literature values [210, 211]. 


\section{Fabry-Perot etalon effects}

\section{D.1. Cryostat windows}

In order to perform low-temperature measurements, the sample is placed inside a Janis SVT-400 flow cryostat. This allows us to conduct measurements between $300 \mathrm{~K}$ and $1.8 \mathrm{~K}$.

When measuring in a standard Janis SVT-400 cryostat, light transients are passing six windows, three when entering and three when exiting the cryostat. The innermost windows are mounted between the sample space (He atmosphere) and the isolation vacuum. The two middle windows are inside the isolation vacuum, as they are part of the liquid-nitrogen heat shielding. They are used to reduce radiation losses. Since these two windows are not essential for the cryogenic operation, the author removed them for performing the time-resolved $\mathrm{THz}$ experiments. In order to have the highest possible $\mathrm{THz}$ transmission, the standard cryostat windows were replaced with optically polished Tsurupica (Picarin) windows.

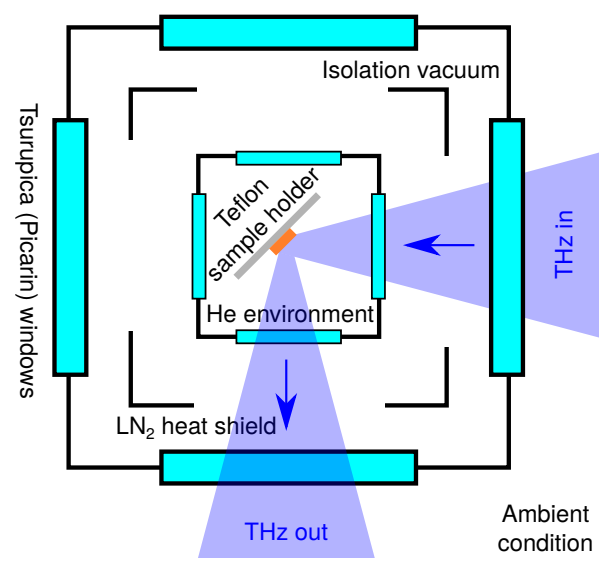

Figure D.1.: Cross section of a Janis SVT-400 cryostat. In normal operation mode, the sample is shielded by three layers of windows. For the T-ray experiments, the author removed the intermediate windows to have less etalon effects, see section D.2 
The etalon effects caused by the cryostat windows are clearly visible in time-resolved measurements, see section D.2. The four essential windows in the cryostat all have a thickness of several $\mathrm{mm}$.

\section{D.2. Challenges in time-resolved measurements}

A light transient passing a window with a refractive index $n$ causes a so-called etalon effect 212. As soon as the refractive index changes at an interface, a light transient is partially reflected. Therefore, if a medium with two parallel surfaces is passed (e.g. generation and detection crystals or cryostat windows), a temporally delayed copy of the original pulse follows shortly after the original pulse. Because of the generation and detection crystals, all $\mathrm{THz}$ time-domain traces (even without cryostat) show etalon signals originating from the internal T-ray reflection mainly in the detection crystal, but also inside the generation crystal.

If the sample is located inside the cryostat, more etalon effects are added due to the cryostat windows. In the author's experiment, the windows are pairwise identical in thickness and therefore have identical etalon times. Hence, two etalon signatures are observed in the time trace, see Fig. D.2. Due to their thicknesses and refractive indices, the etalon peaks are located $22 \mathrm{ps}$ and $30 \mathrm{ps}$ after the incident T-ray pulse. The etalon delay time $\tau_{\text {etalon }}$ can be calculated using Eq. D.1.

$$
\tau_{\text {etalon }}=\frac{2 \cdot n_{\mathrm{THz}} D}{c}
$$

where $D$ depicts the medium thickness, $n_{\mathrm{THz}}$ the refractive index and $c$ the speed of light.

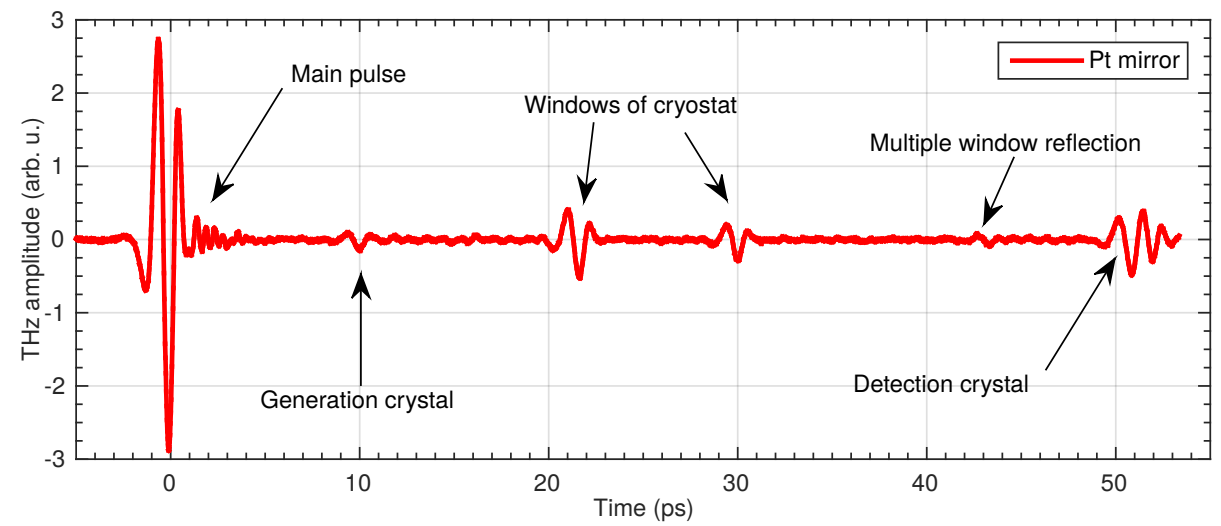

Figure D.2.: Full time trace measured on a platinum reference. Multiple $\mathrm{THz}$ reflections due to etalon effects can be observed. Note that the first etalon peak occurs at $\sim 10$ ps. 
For the $\mathrm{THz}$ generation the author used a $0.5 \mathrm{~mm}$ thick $\mathrm{ZnTe}(110)$ crystal. As detection crystal also a $0.5 \mathrm{~mm} \mathrm{ZnTe}(110)$ crystal was used. In order to significantly increase the etalon time $\tau_{\text {etalon}}$, an optically bound $2 \mathrm{~mm} \mathrm{ZnTe}(100)$ crystal is added to the detection crystal. In terms of electro-optical sampling, only the $0.5 \mathrm{~mm}$ $\mathrm{ZnTe}(110)$ is electro-optically active. Because the refractive indices for $\mathrm{ZnTe}(110)$ and $\mathrm{ZnTe}(100)$ are equivalent, the interface between the two crystal orientations is non-reflecting. With the combined ZnTe detection crystal, the etalon delay time $\tau_{\text {etalon }}$ can be shifted from from $\sim 10$ ps to $\sim 50$ ps. In Fig. D.2, also the etalon signals caused by the cryostat windows are observed.

Since electro-optical sampling is extremely sensitive to electric fields, even multiple internal reflections can also be resolved. If a time trace with an etalon pulse is being Fourier transformed, fringes in the spectrum are expected due to the phase mismatch of the pulses, see Fig. D.3.
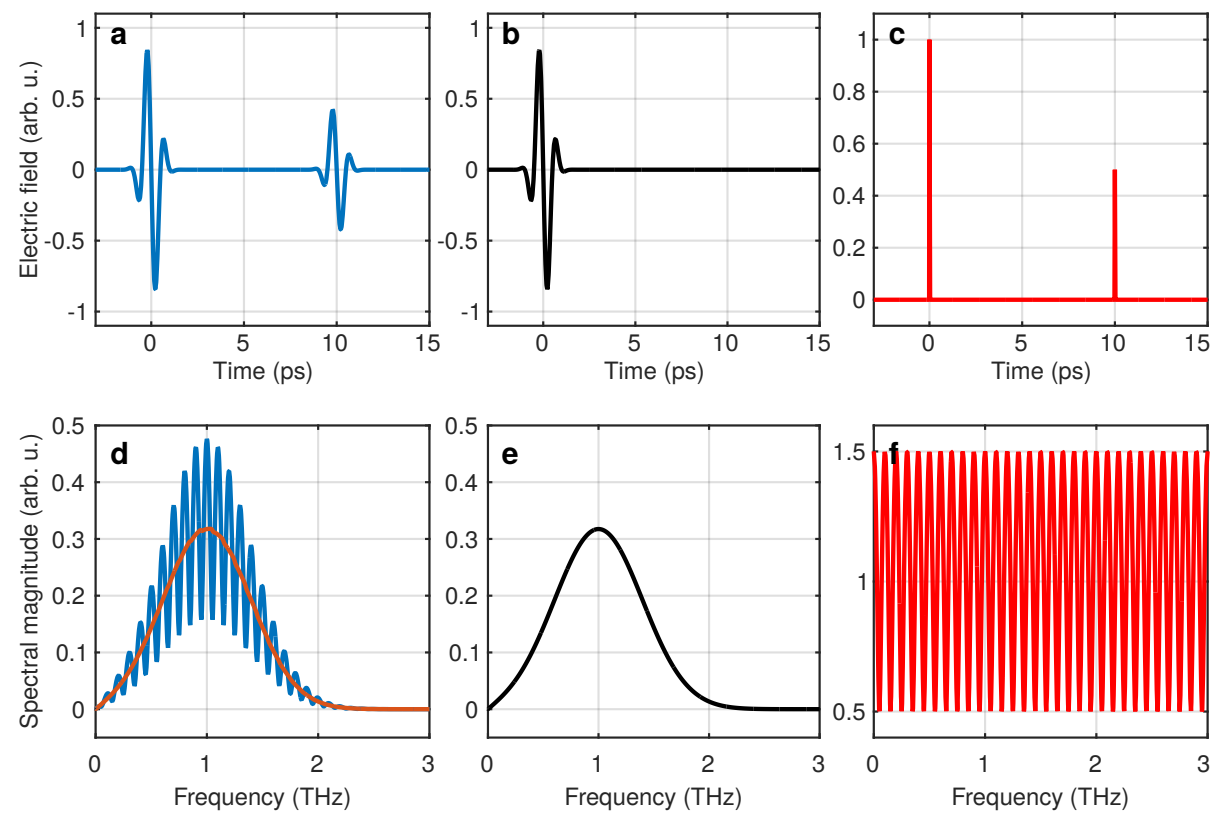

Figure D.3.: (a) Realistic time-domain signal with an etalon reflection at $10 \mathrm{ps}$. The signal is the convolution of the true pulse $E_{\text {true }}$ with the delta peak distribution shown in (c). (b) Original T-ray pulse $E_{\text {true }}(t)$. (c) Peaks at the position where the signals appear. (d) Fourier transform of the realistic time domain. The oscillation originate from the $10 \mathrm{ps}$ retarded signal. The red line is the etalon-corrected signal [213]. (e,f) Fourier transform of the corresponding time signals in (b) and (c). For more details, see text.

The etalon fringes in the spectrum can be mathematically corrected. To do this correction ansatz, the acquired $\mathrm{THz}$ signal $E(t)$ is written as [213]: 


$$
E(t)=\left[\sum_{n=0}^{\infty} M^{n} \delta\left(t-n \tau_{\text {etalon }}\right)\right] * E_{\text {true }}(t)
$$

where $E_{\text {true }}(t)$ is the pure T-ray transient without etalon effects and $\tau_{\text {etalon }}$ is the temporal retardation, see Eq. D.1. $M<1$ depicts the attenuation for every internal reflection process. Hence, in Fourier space $E_{\text {true }}(\omega)$ can be directly extracted [213]:

$$
E_{\text {true }}(\omega)=\frac{\mathcal{F}[E(t)]}{\mathcal{F}\left[\sum_{n=0}^{\infty} M^{n} \delta\left(t-n \tau_{\text {etalon }}\right)\right]},
$$

where $\mathcal{F}$ depicts the Fourier transform. Alternatively, the time trace can be restricted to the first couple of ps (before the first etalon peak at $\sim 10 \mathrm{ps}$ ) in order to avoid artificial fringes in the first place. However, this comprises the spectral resolution. For strong correlation dynamics, the interest is in delay times around a few ps, which is well prior to the first etalon peak. Hence, the author did not have to correct for any etalon effects, as illustrated above, as the time traces were cut before the first etalon signature appears. 


\section{E. Rate equation describing the heavy-fermion dynamics}

As explained in section 6.4 the dynamics of strongly correlated systems can be described by the following rate equation:

$$
\frac{\mathrm{d} n}{\mathrm{~d} t}=-\frac{4 \pi}{\tau_{\mathrm{K}}^{*}}(1-n) \cdot n
$$

where $n(t)$ is the time-dependent, normalized number of photoexcited electrons and $\tau_{\mathrm{K}}^{*}$ the characteristic time scale of the system. This first-order, quadratic differential equation can be solved by separating the variables:

$$
-\frac{4 \pi}{\tau_{\mathrm{K}}^{*}} \mathrm{~d} t=\frac{1}{(1-n) \cdot n} \mathrm{~d} n
$$

Now, Eq. E.2 has to be integrated:

$$
\begin{aligned}
-\frac{4 \pi}{\tau_{\mathrm{K}}^{*}} t+t_{0} & =\int \underbrace{\left(\frac{1}{1-n}+\frac{1}{n}\right)}_{=\frac{1}{(1-n) \cdot n}=\frac{n+(1-n)}{(1-n) \cdot n}} \mathrm{~d} n=-\ln (1-n)+\ln (n)+c_{0} \\
& =\ln \left(\frac{n}{1-n}\right)+c_{0} .
\end{aligned}
$$

Furthermore, Eq. E.3 can be rewritten as:

$$
e^{-\frac{4 \pi}{\tau_{\mathrm{K}}^{*}} t} e^{t_{0}}=\frac{n}{1-n} \cdot c_{1}
$$

where $c_{1}=e^{c_{0}}$. Eq. E.4 can be rearranged as follows:

$$
(1-n) \cdot e^{-\frac{4 \pi}{\tau_{\mathrm{K}}^{*}} t} e^{t_{0}}=c_{1} \cdot n
$$

Note that $e^{t_{0}}$ as well as $c_{1}$ are prefactors, which can be combined to $e^{\frac{4 \pi}{\tau_{\mathrm{K}}^{*}} t_{1}}:=e^{t_{0}} / c_{1}$. From Eq. E.5 the solution $n(t)$ can be directly extracted: 


$$
n(t)=\frac{e^{-\frac{4 \pi}{\tau_{\mathrm{K}}^{*}}\left(t-t_{1}\right)}}{1+e^{-\frac{4 \pi}{\tau_{\mathrm{K}}^{*}}\left(t-t_{1}\right)}}=\frac{e^{-\frac{2 \pi}{\tau_{\mathrm{K}}^{*}}\left(t-t_{1}\right)}}{e^{\frac{2 \pi}{\tau_{\mathrm{K}}^{*}}\left(t-t_{1}\right)}+e^{-\frac{2 \pi}{\tau_{\mathrm{K}}^{*}}\left(t-t_{1}\right)}}=\frac{e^{-x}}{e^{x}+e^{-x}},
$$

where $\frac{2 \pi}{\tau_{\mathrm{K}}^{*}}\left(t-t_{1}\right)$ is substituted by $x$. Eq. E.6 can be rewritten as:

$$
n(t)=\frac{\frac{1}{2}\left(e^{x}+e^{-x}-e^{x}+e^{-x}\right)}{e^{x}+e^{-x}}=\frac{1}{2}-\frac{1}{2} \frac{e^{x}-e^{-x}}{e^{x}+e^{-x}}=-\frac{1}{2} \tanh x+\frac{1}{2} .
$$

Since in Kondo physics the only system parameter is the effective Kondo temperature $T_{\mathrm{K}}^{*}$, there is also only one characteristic time scale: $\tau_{\mathrm{K}}^{*}=\frac{h}{k_{\mathrm{B}} T_{\mathrm{K}}^{*}}$. Therefore, in Eq. E.7 the time constant $t_{1}$ is equal to $\tau_{\mathrm{K}}^{*} / 2 \pi \mathbb{1}^{1}$ Hence, the resulting time-dependent number of photoexcited electrons $n(t)$ reads as follows:

$$
n(t)=-\frac{1}{2} \tanh \left(\frac{2 \pi}{\tau_{\mathrm{K}}^{*}}\left(t-\frac{\tau_{\mathrm{K}}^{*}}{2 \pi}\right)\right)+\frac{1}{2}=-\frac{1}{2} \tanh \left(\frac{2 \pi t}{\tau_{\mathrm{K}}^{*}}-1\right)+\frac{1}{2} .
$$

which corresponds to Eq. 6.9.

\footnotetext{
${ }^{1}$ The factor $1 / 2 \pi$ has to be introduced because of the definition of $x$.
} 


\section{F. Resistivity analysis}

As explained in section 4.3.1, the Kondo effect has a profound influence on thermodynamic quantities such as the resistivity $R$ [31, 214]. As explained there, the optical resistivity of heavy-fermion systems has been investigated also in the $\mathrm{THz}$ range using the energetically highly-resolved THz-FDS [109, 197, 215, 218, In fact, the $T^{2}$ dependence of the electrical resistivity $R$ is an often used criterion to indicate Fermi-liquid behavior in heavy fermions [109]:

$$
R_{\mathrm{HF}}(T)=R_{0}+\rho_{1} T^{2}+R_{3} \ln \frac{1}{T}
$$

Another signature of heavy fermions is a relatively temperature-independent resistivity at high temperatures. In THz-TDS, if the frequency resolution is not of interest, the peak value of the conduction electron response can be analyzed, as it corresponds to the optical resistivity averaged over the pulse spectrum (here: between 0.3 and $3 \mathrm{THz}$ ). The measured optical resistivity of $\mathrm{CeCu}_{5.9} \mathrm{Au}_{0.1}$ is shown in Fig. F.1. The heavy-fermion behavior, as described by Eq. F.1, is in good agreement with the measurement.

The drop in the resistivity below $3 \mathrm{~K}$ indicates the Kondo-lattice coherence temperature, see section 4.3.1. Because the investigated $\mathrm{CeCu}_{5.9} \mathrm{Au}_{0.1}$ sample is also quantum critical, the question of the influence of the QCP arises. The QCP manifests in a vanishing Kondo spectral weight, as shown in Fig. 7.3 . Hence, quantum criticality dominates the sample below $5 \mathrm{~K}$. However, the resistivity in this temperature range is still logarithmically diverging. Therefore, the onset of quantum criticality and the Kondo-lattice coherence signature are most likely two different effects observed. 


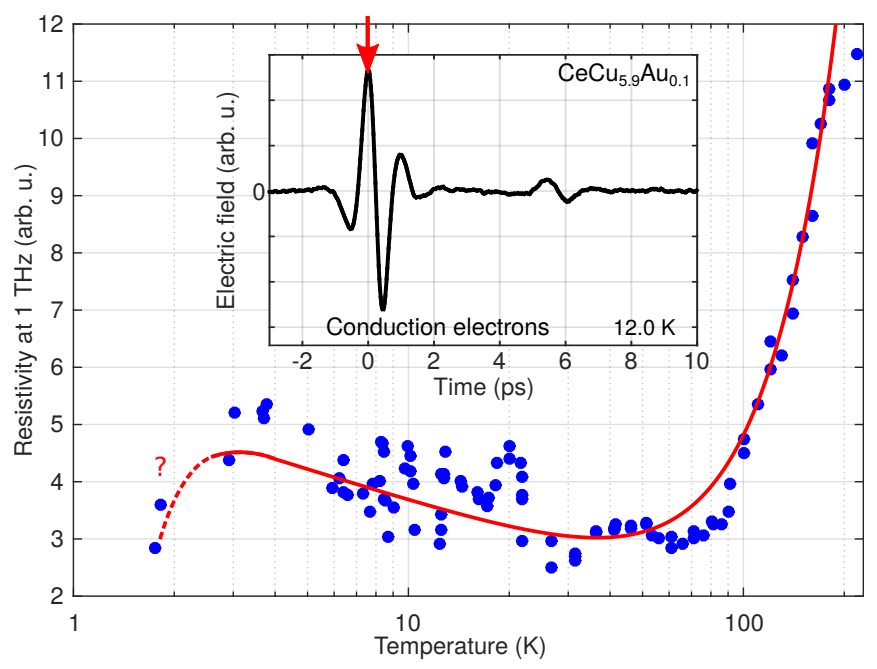

Figure F.1.: The temperature-dependent resistivity $R(T)$ extracted from the maximum value of conduction electron response (blue points). The resistivity has a quadratic temperature dependence for temperatures above approximately $50 \mathrm{~K}$. The logarithmic divergence is dominating between 3 and $50 \mathrm{~K}$. At very low temperatures $(<3 \mathrm{~K})$, the resistivity drops again. Most likely, this depicts the onset of Kondo-lattice coherence [71, 110]. The solid red curve depicts the behavior illustrated in Eq. F.1. The dotted red curve illustrates the resistivity decrease towards $0 \mathrm{~K}$. Hence, a Kondo-lattice coherence temperature of approximately $3 \mathrm{~K}$ is observed. Inset: The red arrow illustrates which value is used to extract the resistivity data.

\section{F.1. Kondo lattice vs. Kondo impurity at low temperatures}

The temperature-dependent resistivity discussed so far is valid for the Kondo-impurity model as well as for Kondo lattices. However, the major difference between a Kondo impurity and a Kondo-lattice effect manifests at very low temperatures [71, 110]. At temperatures below the Kondo temperature, the resistivity is logarithmically diverging. This scenario is valid for the Kondo impurity as well as for the Kondo lattice. However, as the temperature approaches $0 \mathrm{~K}$, the two systems start to behave qualitatively differently. In the Kondo-impurity case, the resistivity remains on a constant level. However, in a Kondo lattice a lattice-coherent ground state establishes, which manifests in an again decreasing resistivity, see also Fig. 4.7. The data shown in Fig. F.1 indicate the onset of this lattice-coherent onset at approximately $3 \mathrm{~K}$. The fact that also the conduction electron signature contains the Kondo-lattice information corroborates the coherent Kondo dynamics proposed in the thesis. 


\section{G. Data overview}

\begin{tabular}{|c|c|c|}
\hline Fig. number & data file & date \\
\hline Fig. 5.4 & time_0.dat & 24.3 .2015 \\
\hline Fig. 6.1 & $\begin{array}{l}\text { time_9.dat } \\
\text { time_8.dat } \\
\text { time_12.dat }\end{array}$ & $\begin{array}{l}25.3 .2015 \\
24.3 .2015 \\
26.2 .2015\end{array}$ \\
\hline Fig. 6.2 & time_9.dat & 24.3 .2015 \\
\hline Fig. 6.6 & time_0.dat - time_12.dat & $24.3 .2015-27.3 .2015$ \\
\hline Fig. $\overline{6.8}$ & $\begin{array}{l}\text { time_4.dat } \\
\text { time_9.dat }\end{array}$ & $\begin{array}{l}5.8 .2016 \\
25.3 .2015\end{array}$ \\
\hline Fig. 6.9 & time_4.dat, time_5.dat & 5.8 .2016 \\
\hline Fig. 6.10 & time_0.dat-time_15.dat & 22.8 .2016 \\
\hline Fig. 6.11 & time_0.dat-time_12.dat & $24.3 .2015-27.3 .2015$ \\
\hline Fig. 6.12 & time_0.dat-time_12.dat & $24.3 .2015-27.3 .2015$ \\
\hline Fig. 6.13 & time_0.dat-time_12.dat & $24.3 .2015-27.3 .2015$ \\
\hline Fig. 6.14 & time_0.dat-time_12.dat & $24.3 .2015-27.3 .2015$ \\
\hline Fig. 6.15 & time_0.dat - time_12.dat & $24.3 .2015-27.3 .2015$ \\
\hline Fig. 6.16 & time_0.dat-time_11.dat & $21.9 .2015-24.9 .2015$ \\
\hline Fig. 6.17 & time_0.dat-time_11.dat & $21.9 .2015-24.9 .2015$ \\
\hline Fig. 7.1 & time_0.dat - time_12.dat & $24.3 .2015-27.3 .2015$ \\
\hline Fig. 7.2 & $\begin{array}{l}\text { time_0.dat-time_27.dat } \\
\text { time_0.dat-time_19.dat } \\
\text { time_0.dat-time_39.dat } \\
\text { time_0.dat-time_30.dat } \\
\text { time_0.dat-time_41.dat }\end{array}$ & $\begin{array}{l}1.9 .2016 \\
2.9 .2016 \\
5.9 .2016 \\
6.9 .2016 \\
7.9 .2016-8.9 .2016\end{array}$ \\
\hline Fig. 7.3 & $\begin{array}{l}\text { time_0.dat-time_27.dat } \\
\text { time_0.dat-time_19.dat } \\
\text { time_0.dat-time_39.dat } \\
\text { time_0.dat-time_30.dat } \\
\text { time_0.dat-time_41.dat }\end{array}$ & $\begin{array}{l}1.9 .2016 \\
2.9 .2016 \\
5.9 .2016 \\
6.9 .2016 \\
7.9 .2016-8.9 .2016\end{array}$ \\
\hline
\end{tabular}





\section{H. Spatial resolution in $\chi^{(2)}$-processes}

Second-order optical processes, such as the $\mathrm{THz}$ generation or second-harmonic generation (SHG), are strongly depending on the crystal symmetry. Specifically, they are only allowed in non-centrosymmetric crystals. The intensity of the generated light is strongly depending on the phase matching between the pump beam and the generated radiation, see section 5.4. For improper phase matching, the intensity of the generated radiation is periodically modulated, see section 5.4.1. The phase-matching condition can be improved by tilting the generation crystal, which compensates the velocity mismatch geometrically. Alternatively, the phase matching in the generation crystal can be achieved by periodically poling the crystal along the direction of propagation. As the sign of the order parameter changes, the generated light picks up an additional phase $\pi$. Hence, the destructive interference can be suppressed.

The order-parameter sensitivity of the SHG is a major advantage for domain imaging. As a result, a domain pattern can be spatially resolved using SHG, as the domain walls between two domains appear as dark lines. The reason is the destructive interference of the locally generated SHG light in close proximity of the atomically sharp domain wall. Due to the wave nature of light, the diffraction-limited optical resolution is given by an area called airy disk $A$. Hence, the thickness of the domain wall depends on the optical resolution, see Fig. H.1.

In the hypothetical case of perfect optical resolution, the airy disk is infinitesimally small and therefore the domain walls are invisible as no waves destructively interfere. Hence, the number of domain walls as well as the airy-disk size have an influence on the integrated SHG intensity emitted from a multi-domain sample. Consequently, the normalized SHG intensity $\eta$ can be defined as follows:

$$
\eta:=\frac{\int_{\text {sample }} I_{\mathrm{SHG}}^{\text {multi domain }}(\vec{x}) \mathrm{d} \vec{x}}{\int_{\text {sample }} I_{\mathrm{SHG}}^{\text {single domain }}(\vec{x}) \mathrm{d} \vec{x}},
$$

where the integrated SHG intensity of the multi-domain state is normalized to the integrated SHG intensity of the single-domain state 1 The normalized SHG intensity $\eta$ can take values between 0 and 1 . For simplicity, we assume only two

${ }^{1}$ The integrated SHG intensity of an image with infinitesimally good optical resolution is identical to the SHG intensity of a single-domain state. 


\section{H.1. Analytical calculation of integrated SHG intensity for large domains}

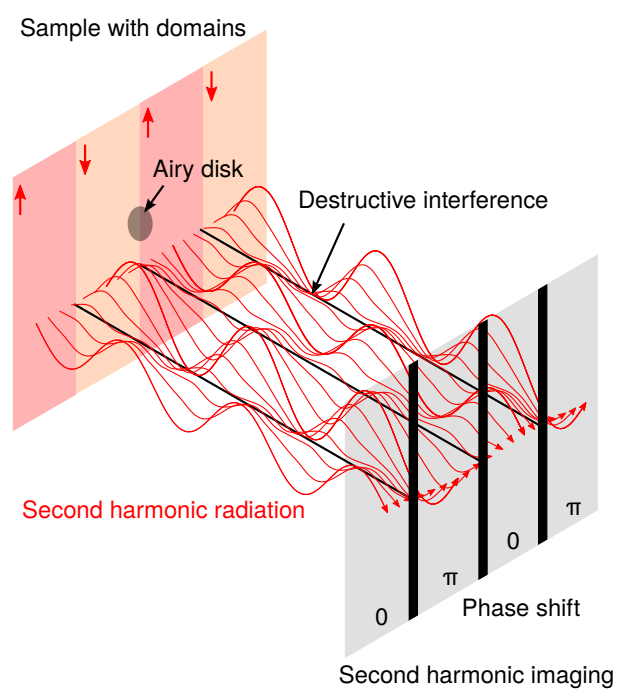

Figure H.1: Illustration of SHG imaging. Areas with opposite order parameters emit SHG light with a relative phase shift of $\pi$. Therefore, in SHG images the domain walls appear as dark areas due to the destructive interference. The thickness of the domain walls in the image depends on the optical resolution, which is given by the airy disk.

domain types:,$+^{6}$ and,$-^{6}$. Hence, the spatially resolved normalized SHG intensity $\eta(x)$ can be defined as follows:

$$
\eta(\vec{x})=\frac{D_{+}-D_{-}}{A}
$$

where $D_{+}$and $D_{-}$label the areas of the two domain types within the airy disk surrounding the coordinate $\vec{x}$ on the sample. In the case of equal areas, the local SHG emission is fully suppressed and $\eta(\vec{x})=0$. In the case that only one domain type is within the airy disk, the local SHG intensity is $\eta(\vec{x})=1$.

The integrated and normalized SHG intensity $\eta$ depends on the domain size, as it sums up all the locally measured imbalances between the two domain types. Hence, the ,cluster size'of same domain-type areas are also captured by the integrated SHG intensity. In fact, $\eta$ can even predict cluster sizes which are even orders of magnitude below the optical resolution, see H.3

\section{H.1. Analytical calculation of integrated SHG intensity for large domains}

For simplicity, a quadratic airy disk with an edge length $a$ is assumed ${ }^{2}$ Therefore, the airy disk $A$ corresponds to $a^{2}$. The sample can be divided into stripes of width $a$, on which the domain walls appear as straight lines. They can be further assumed to be perpendicular to the stripe, see Fig. $\mathrm{H} .2$. Note that the following discussion

\footnotetext{
${ }^{2}$ Implementing a circular airy-disk shape is also possible.
} 


\section{H.1. Analytical calculation of integrated SHG intensity for large domains}

in this section is only valid for large domains. Large domains in this context are linked to the following condition:

$$
\langle d\rangle \geq a
$$

where $\langle d\rangle$ is the average distance between two domain walls. The integrated SHG intensity can be calculated as illustrated in Fig. H.2.

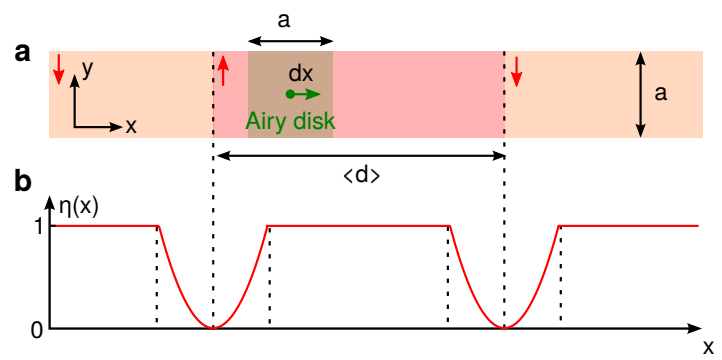

Figure H.2.: Illustration of the large-domain case. (a) The sample can be regarded as a stripe with a perpendicular domain pattern. The distance between domain walls is $\langle d\rangle$ and the stripe width is chosen to be equal to the edge length $a$ of the airy disk. (b) As the airy disk is shifted in x-direction across the stripe pattern, the local SHG intensity $\eta(x)$ varies. Far from the domain walls, $\eta(x)$ is 1 . In the vicinity of the domain walls, $\eta(x)$ is attenuated as some SHG emitted from the two domain areas destructively interfere, see Eq. H.2. By integrating along $x$, the integrated SHG intensity $\eta$ can be estimated.

Multi-domain case. In average, a domain wall appears after a distance $\langle d\rangle$. In between two domain walls, the locally generated SHG intensity is not suppressed. Hence, the following integral describes the multi-domain case:

$$
\begin{aligned}
I_{\mathrm{SHG}}^{\mathrm{multi} d o m a i n} & =\frac{1}{a}\left[\int_{0}^{a} \mathrm{~d} y \int_{a / 2}^{\langle d\rangle-a / 2} a \mathrm{~d} x+\int_{0}^{a} \mathrm{~d} y 2 \int_{0}^{\frac{a}{2}} 2 x \mathrm{~d} x\right] \\
& =a[\langle d\rangle-a]+2\left[x^{2}\right]_{0}^{a / 2}=a[\langle d\rangle a]+\left[a^{2} / 2\right] .
\end{aligned}
$$

Single-domain case. In the single-domain case there are no domain walls on the entire sample. Therefore the single-domain SHG intensity, for the same sample stripe used in the multi-domain case, can be calculated as follows:

$$
I_{\mathrm{SHG}}^{\text {single domain }}=\int_{0}^{a} \mathrm{~d} y \int_{0}^{\langle d\rangle} \mathrm{d} x=a\langle d\rangle \quad .
$$


Summary In the limit of large domains, the normalized SHG intensity $\eta$ is given by the following equation:

$$
\eta^{>}:=\frac{a[\langle d\rangle-a]+\left[a^{2} / 2\right]}{a\langle d\rangle}=\frac{\langle d\rangle-a / 2}{\langle d\rangle}=1-\frac{a}{2\langle d\rangle} \quad, \quad \text { for } \quad\langle d\rangle \geq a
$$

Note that for the two limits $\langle d\rangle \rightarrow \infty$ and $\langle d\rangle \rightarrow a, \eta$ takes the following values:

$$
\begin{aligned}
\lim _{\langle d\rangle \rightarrow \infty} \eta^{>} & =\lim _{\langle d\rangle \rightarrow \infty} 1-\frac{a}{2 d}=1 \\
\lim _{\langle d\rangle \rightarrow a} \eta^{>} & =\lim _{\langle d\rangle \rightarrow a} 1-\frac{a}{2 d}=1 / 2
\end{aligned} .
$$

For $\langle d\rangle \leq a$ and a stripe of width $a$, a two-dimensional model has to be considered as the previous assumption of only perpendicular stripes in an isotropic system breaks down. Alternatively, the stripe width can be reduced from $a$ to $\langle d\rangle$, see section H.2.

\section{H.2. Analytical calculation of integrated SHG intensity for small domains}

In the limit of small domains, $\langle d\rangle \leq a$, the stripe width has to be renormalized. If the stripe width is reduced to $\langle d\rangle$, again only perpendicular domain walls along the y-axis can be assumed. However, the airy disk now includes many domain walls and therefore, the SHG generation is strongly suppressed. In this limit, only small contributions towards the SHG generation can be found in specific configurations, where the two domain areas are not balanced within the airy disk. However, as the airy disk is again translated over the distance $\langle d\rangle$ of the sample, the intensity will still be modified in a parabolic manner. The small-domain case is illustrated in Fig. H.3.

Multi-domain case. A multi-domain structure in the limit of small domains is captured by Fig. H.3. Similar to the large-domain case, the translation over a distance $\langle d\rangle$ has to be taken into account, as this takes the periodicity of the domain structure into account. However, in contrast to the large-domain case, the local SHG intensity in between domain walls never reaches 1 . Therefore, the following integral expression describes the small-domain case:

$$
I_{\mathrm{SHG}}^{\text {multi domain }} \approx \int_{0}^{\langle d\rangle} x \mathrm{~d} x=\frac{\langle d\rangle^{2}}{2}
$$




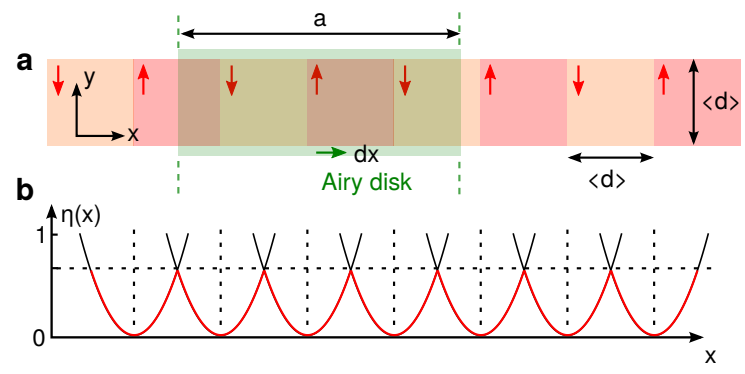

Figure H.3.: Illustration of the small-domain case. (a) The sample can be regarded as a stripe with a perpendicular domain pattern. The distance between domain walls is $\langle d\rangle$ and the stripe width is chosen to be $\langle d\rangle$. In the small-domain case, the airy disk is much bigger than the stripe pattern itself. (b) As the airy disk is shifted across the sample, the local SHG intensity $\eta(x)$ varies. In the small-domain case illustrated here, $\eta(x)$ is always smaller than 1 as for any position most of the SHG light is destructively interfering, see Eq. H.2. By integrating over the distance $\langle d\rangle$, the integrated SHG intensity $\eta$ can be estimated.

Single-domain case. The corresponding single-domain case is described by the following integral, which is required to extract the normalized SHG intensity $\eta$ :

$$
I_{\mathrm{SHG}}^{\text {single domain }}=\langle d\rangle \int_{0}^{a} \mathrm{~d} x=\langle d\rangle a .
$$

Summary. The resulting SHG intensity $\eta$ in the limit of small domains is given with the following equation:

$$
\eta^{<}:=\frac{\langle d\rangle^{2} / 2}{\langle d\rangle a}=\frac{1}{2} \frac{\langle d\rangle}{a}
$$

Note that for the two limits $\langle d\rangle \rightarrow 0$ and $\langle d\rangle \rightarrow a, \eta$ takes the following values:

$$
\begin{aligned}
\lim _{\langle d\rangle \rightarrow 0} \eta^{<} & =\lim _{\langle d\rangle \rightarrow 0} \frac{\langle d\rangle}{2 a}=0 \\
\lim _{\langle d\rangle \rightarrow a} \eta^{<} & =\lim _{\langle d\rangle \rightarrow a} \frac{\langle d\rangle}{2 a}=1 / 2 .
\end{aligned}
$$

Hence, the two models for the large and the small-domain case match in the crossover region. 


\section{H.3. Final remarks}

The integrated SHG intensity can be used to extract information about the cluster size of domain areas, even well below the optical resolution. The previously derived model allows us to estimate $\langle d\rangle$ as function of the integrated SHG intensity $\eta$ and the optical resolution $a$. If the domain size equals the optical resolution $\langle d\rangle=$ $a$, the normalized SHG intensity is expected to be $1 / 2$. If the normalized SHG intensity is below this threshold, the domain structure is smaller than the optical resolution and is therefore in the small-domain regime. The model calculation, given in section H.2 allows us to estimate the domain size $\langle d\rangle$, even if the individual domain walls can no longer be spatially resolved. Also in the large-domain regime, the model allows us to estimate $\langle d\rangle$ as function of $\eta$.

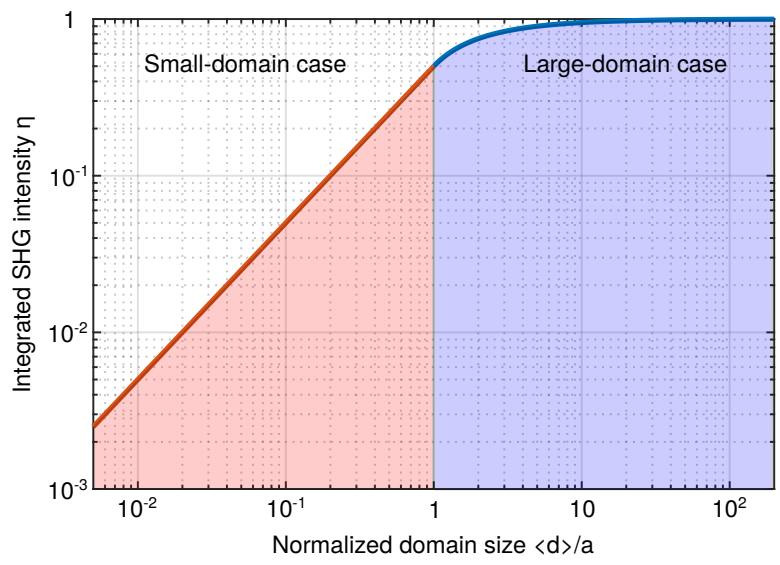

Figure H.4: $\quad$ Integrated SHG intensity $\quad \eta$ as function of the normalized domain size $\langle d\rangle / a$, where $a$ is the edge length of a quadratically assumed airy disk. The reddish part describes the normalized SHG intensity in the sub-resolution domain regime. A linear behavior in the normalized domain size $\langle d\rangle / a$ is expected. The blue part describes the normalized SHG intensity for the regime, where the domains can be optically resolved. In this part, the SHG intensity $\eta$ is described by a $\frac{x-1}{x}$ behavior.

Note that the proposed integrated SHG model has to be experimentally verified. Numerical Monte-Carlo simulations indicate the correctness of the model proposed in this section. The analytical model allows us to extract the normalized domain size $\langle d\rangle / a$ as function of the integrated and normalized SHG intensity $\eta$. In fact, the model gives access to domain information, even for structures which are orders of magnitude below the optical resolution. 


\section{Scientific achievements}

\section{Publications}

7. C. Wetli, J. Kroha, O. Stockert, H. v. Löhneysen, K. Kliemt, C. Krellner and M. Fiebig,

Time-resolved collapse and revival of the Kondo state near a quantum phase transition, (submitted)

6. C. Wetli, J. Kroha, O. Stockert, H. v. Löhneysen, K. Kliemt, C. Krellner and M. Fiebig,

Time-resolved collapse and revival of the Kondo state near a quantum phase transition, arXiv:1703.04443 e-prints (2017)

5. C. Wetli, V. Wegmayr, T. Lottermoser and M. Fiebig, Sub-resolution domain imaging using second harmonic generation, (in preparation for submission)

A. Fognini, G. Salvatella, T. U. Michlmayr, C. Wetli,

U. Ramsperger, T. Bähler, F. Sorgenfrei, M. Beye,

4. A. Eschenlohr, N. Pontius, C. Stamm, F. Hieke, M. Dell'Angela,

S. de Jong, R. Kukreja, N. Gerasimova, V. Rybnikov, H. Redlin, J. Raabe, A. Föhlisch, H. A. Dürr, W. Wurth, D. Pescia,

A. Vaterlaus and Y. Acremann,

The role of space charge in spin-resolved photoemission experiments,

New J. Phys., 16, 043031 (2014)

A. Fognini, T. U. Michlmayr, G. Salvatella, C. Wetli,

U. Ramsperger, T. Bähler, F. Sorgenfrei, M. Beye,

3. A. Eschenlohr, N. Pontius, C. Stamm, F. Hieke, M. Dell'Angela, S. de Jong, R. Kukreja, N. Gerasimova, V. Rybnikov,

A. Al-Shemmary, H. Redlin, J. Raabe, A. Föhlisch, H. A. Dürr, W. Wurth, D. Pescia, A. Vaterlaus and Y. Acremann, Ultrafast reduction of the total magnetization in iron, Appl. Phys. Lett. 104, 032402 (2014) 
2. T. U. Michlmayr, A. Fognini, T. Bähler, G. O. Salvatella, C. Wetli, A. Vaterlaus and Y. Acremann, A mobile UHV-system designed for femtosecond time, energy and spin resolved photoelectron spectroscopy, J. Kor. Phys. 62, 12 (2013)

1. A. Fognini, T. U. Michlmayr, T. Bähler, C. Wetli, A. Vaterlaus and Y. Acremann,

Magnetic pulser and sample holder for time- and spin-resolved photoemission spectroscopy on magnetic material,

Rev. Sc. In., 83, 063906 (2012) 


\section{Invited talks}

January 2017 Invited talk at the IPK Colloquium

at the Karlsruhe Institute of Technology,

Karlsruhe, Germany

Host: Prof. Dr. Hilbert von Löhneysen

January 2017 Invited talk at the trans-regional Colloquium (TRR80)

at the Augsburg University,

Augsburg, Germany

Host: Prof. Dr. Philipp Gegenwart

\section{Conference contributions}

March 2017 Talk at DPG Spring Meeting,

Dresden, Germany

March $2016 \quad$ Talk at APS March Meeting,

Baltimore, United States of America

March $2016 \quad$ Talk at DPG Spring Meeting,

Regensburg, Germany

September 2015 Poster presentation at Scientifica 2015,

Zurich, Switzerland

June 2015 Poster presentation at MaP Graduate Symposium,

Zurich, Switzerland

March 2015 Talk at DPG Spring Meeting,

Berlin, Germany

March 2015 Poster presentation at DPG Spring Meeting,

Berlin, Germany 



\section{Curriculum vitae}

Omitted in the electronic version. 